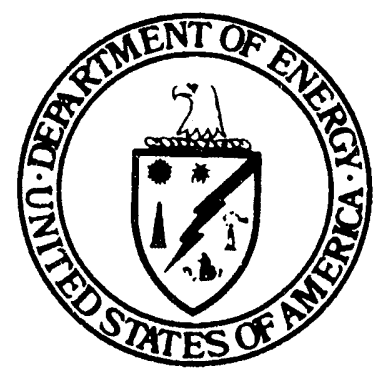

\title{
Summaries of FY 1992 Research in Nuclear Physics
}

July 1993

U. S. Department of Energy Office of Energy Research Office of High Energy and Nuclear Physics Division of Nuclear Physics Washington, D.C. 20585

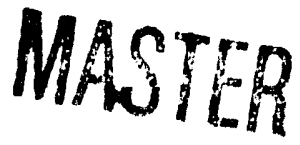


This report summarizes the research projects supported by the Division of Nuclear Physics in the Office of High Energy and Nuclear Physics during FY 1992. This Division is a component of the Office of Energy Research and provides about $85 \%$ of the funding for nuclear physics research in the United States. The objectives of the Nuclear Physics Program are two-fold: (1) to understand the interactions and structures of atomic nuclei and nuclear matter and the fundamental forces of nature as manifested in nuclear matter and (2) to foster application of this knowledge to other sciences and technical disciplines. These summaries are intended to provide a convenient guide for those interested in the research supported by the Division of Nuclear Physics.

We remind the readers that this compilation is just an overview of the Nuclear Physics Program. What we attempt to portray correctly is the breadth of the program and level of activity in the field of nuclear physics research as well as the new capabilities and directions that continually alter the public face of the nuclear sciences. We hope that the limitations of space, constraints of format, and rigors of editing have not extinguished the excitement of the science as it was originally portrayed.

David L. Hendrie

Director

Division of Nuclear Physics 
The Nuclear Physics Program conducts fundamental research consisting of experimental and theoretical investigations of atomic nuclei and nuclear matter. Vital to the program are talented and imaginative scientists and research facilities that provide nuclear probes, detector systems, and computational devices needed for modern nuclear research. In FY 1992, 1,428 scientists (1,004 Ph.D. staff and 424 graduate students) were supported by the program.

Along with our sister program in the National Science Foundation, the primary goal of the Nuclear Physics Program is to understand the structure of atomic nuclei and the fundamental forces required to hold their constituents in place. Nuclear processes determine the essential physical characteristics of the cosmos and the relative abundance of the matter out of which it is made. The science of nuclear physics has spawned many diverse technologies such as nuclear medicine, nuclear power, nuclear fusion, and nuclear weapons. These technologies have matured to the point where they now operate almost independently from the basic research out of which they emerged. Nevertheless, vital interactions still occur in the development of advanced concepts, theoretical models, and instrumentation.

Research in the Nuclear Physics Program depends on the existence and effective operation of large and complex accelerator facilities, which are the essential tools of the nuclear scientist. These facilities are made available to all the Nation's scientists on the basis of scientific merit and technical feasibility of proposals reviewed by peer groups called program advisory committees. Nuclear physics accelerators also generate radioisotopes used for medical diagnoses and support several cooperative programs in biomedical research and atomic physics. The Nuclear Physics Program consists of four subprograms: Medium Energy Nuclear Physics, Heavy Ion Nuclear Physics, Low Energy Nuclear Physics, and Nuclear Theory.

The Medium Energy Nuclear Physics subprogram supports nuclear physics research at electron and proton accelerator facilities with sufficient primary beam energy to produce $\pi$ mesons (pions). In addition, this subprogram supports medium energy experiments at accelerators operated by other Department of Energy and National Science Foundation programs and at foreign facilities. Research activities cover a variety of topics that include: (1) the properties of nuclei that can be determined from hadron and lepton probes, (2) the mechanisms by which hadrons interact with nuclei, (3) the properties of the weak and strong interactions, (4) the fundamental symmetries of nature, and (5) the distribution of electric charge and electric currents within nuclei. A new accelerator facility, the Continuous Electron Beam Accelerator Facility (CEBAF), is being constructed at Newport News, Virginia, and is expected to be operational for experiments in 1995. CEBAF will use the electromagnetic interaction as a probe of the nucleons in the transition region between the conventional nucleon-meson picture of nuclear structure and the quark-gluon picture that emerges from quantum chromodynamics.

The Heavy Ion Nuclear Physics subprogram is aimed at understanding the behavior of nuclei and nuclear matter over a wide range of excitation energy, nuclear density, angular monientum, and deformation. These conditions are created in collisions between nuclear targets and nuclear beams of heavy ions (defined as nuclei that contain more than four nucleons). Studies include the behavior of nuclei matter at high spin, which leads to large shape deformation and fission. At low bombarding energies, nuclear orbiting phenomena are studied. Especially intriguing is the unexplained spontaneous 


\section{INTRODUCTION}

production of positrons, which occurs in close encounters of the heaviest nuclei. The nuclear dynamics of complex phenomena, including deep-inelastic scattering and projectile fragmentation, is studied at medium bombarding energies. At higher energies, the nuclear matter equation of state for hot dense nuclear matter is explored. At ultrarelativistic energies, where the nuclei move at nearly the speed of light, a search is being made for a predicted state of matter known as the quark-gluon plasma. A new facility, the Relativistic Heavy Ion Collider (RHIC), is being constructed at Brookhaven National Laboratory with completion expected about 1999. RHIC is a two-ring colliding beam accelerator dedicated to the study of nuclear matter at very high temperatures and densities. At the design energies of RHIC, nuclear matter loses its identity as a collection of neutrons and protons and is predicted to undergo a phase transition to a plasma of quarks and gluons.

The Low Energy Nuclear Physics subprogram emphasizes investigations of nuclear structure, decay parameters of radionuclides, and precision tests of fundamental theories and symmetries. Another research area addresses problems of mutual interest to nuclear physics and astrophysics, including measurements of solar neutrinos and the problem of stellar nuclear abundances. Besides the Radioactive Ion Beam (RIB) project at Oak Ridge National Laboratory, the Low Energy Nuclear Physics subprogram uses electrostatic and cyclotron accelerators to generate probes of polarized and unpolarized light ions, neutrons, and gamma-rays. Also carried under this activity is the Nuclear Data Program, which has as a primary goal the compilation, evaluation, and dissemination of an accurate, complete, and accessible nuclear information base. A secondary component is the measurement of critical quantities that are extensions of the Low Energy Nuclear Physics Program such as cross sections of importance to nuclear astrophysics and deexcitation parameters of short-lived, far from stability nuclei, which are of importance to safety calculations of reactor decay heat.

The Nuclear Theory subprogram strives toward a unified description of atomic nuclei at the most fundamental level. This description is sometimes most suitably made in terms of nucleons (neutrons and protons) and sometimes involves their elementary constituent particles, quarks, and gluons. The objective of nuclear theory is approached by interpreting results from nuclear physics experiments, by predicting phenomena and relationships, and by providing unifying concepts and formulations of diverse experimental observations. Much of nuclear theory research requires extensive use of supercomputer facilities.

The research summaries in this report are intended to describe the general character and goals of the individual research programs, current research efforts, especially significant recent results, and plans for the near future. Primary publications should be used for reference to the work for a more complete and accurate understanding. The research summaries were prepared by the investigators, then reviewed and minimally edited by DOE staff. The names of Ph.D.-level personnel who are associated with the work and the FY 1992 funding level are included. The summaries are organized into two groups: research programs at national laboratories and those at universities, with the material arranged alphabetically by institution. To aid the reader, a glossary of nuclear pinysics terminology and an Index of Investigators are provided at the end of the research summaries.

Questions about the details of the individual research projects in this compilation should be directed to the investigators listed. The staff of the Division of Nuclear Physics will be pleased to respond to questions regarding the Nuclear Physics Program. Our telephone number is (301) 903-3613 and mail address is Division of Nuclear Physics, ER-23, U.S. Department of Energy, Washington, DC 20585. 
We welcome your comments and inquiries.

For more information, contact the following DOE program managers:

- Medium Energy Nuclear Physics-Sherman P. Fivozinsky

- Heavy Ion Nuclear Physics--Dennis G. Kovar

- Low Energy Nuclear Physics-Richard A. Meyer

- Nuclear Data Program-Richard A. Meyer

- Nuclear Theory-Joseph B. McGrory

- Facilities and Instrumentation-John R. Erskine

We appreciate the assistance of Walcoff \& Associates in the preparation of this report.

John R. Erskine

Editor 
Foreword

Introduction $\ldots \ldots \ldots \ldots \ldots \ldots \ldots \ldots \ldots \ldots \ldots \ldots \ldots \ldots \ldots \ldots \ldots$

\section{Research Programs at National Laboratories}

Argonne National Laboratory $\ldots \ldots \ldots \ldots \ldots \ldots \ldots \ldots \ldots \ldots \ldots \ldots \ldots \ldots$

Brookhaven National Laboratory $\ldots \ldots \ldots \ldots \ldots \ldots \ldots \ldots \ldots \ldots \ldots \ldots$

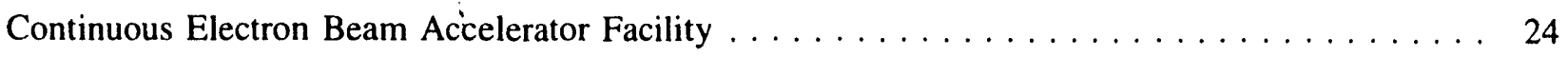

Idaho National Engineering Laboratory $\ldots \ldots \ldots \ldots \ldots \ldots \ldots \ldots \ldots \ldots \ldots \ldots$

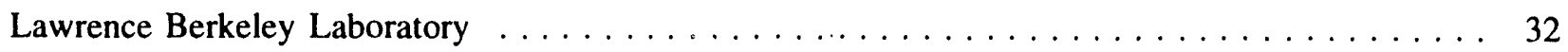

Lawrence Livermore National Laboratory $\ldots \ldots \ldots \ldots \ldots \ldots \ldots \ldots$

Los Alamos National Laboratory $\ldots \ldots \ldots \ldots \ldots \ldots \ldots \ldots \ldots \ldots \ldots$

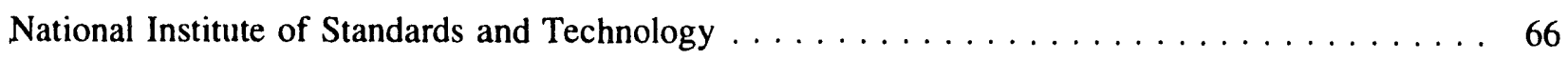

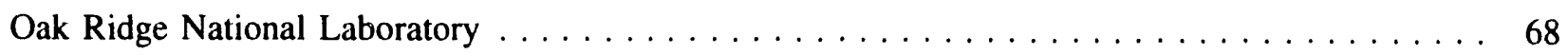

Oak Ridge Institute For Science and Education $\ldots \ldots \ldots \ldots \ldots \ldots \ldots \ldots$

Pacific Northwest Laboratory.$\ldots \ldots \ldots \ldots \ldots \ldots \ldots \ldots \ldots \ldots \ldots$

\section{Research Programs at Universities}

Abilene Christian University . . . . . . . . . . . . . . . . . . . . 79

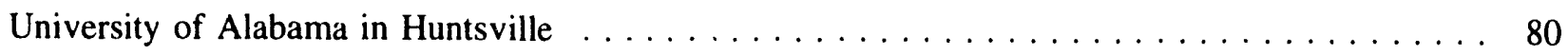

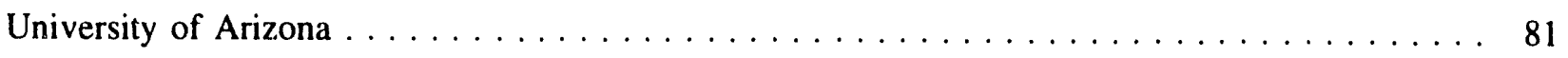

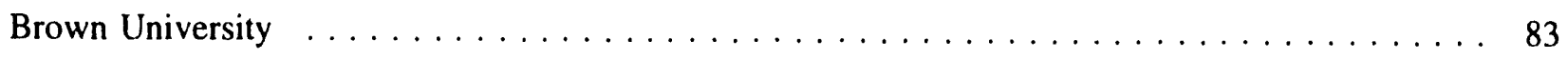

University of California, Berkeley $\ldots \ldots \ldots \ldots \ldots \ldots \ldots \ldots \ldots \ldots \ldots$

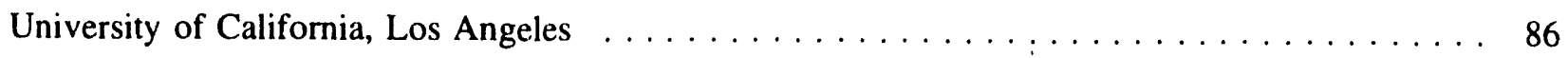

University of California, Riverside $\ldots \ldots \ldots \ldots \ldots \ldots \ldots \ldots \ldots \ldots \ldots$

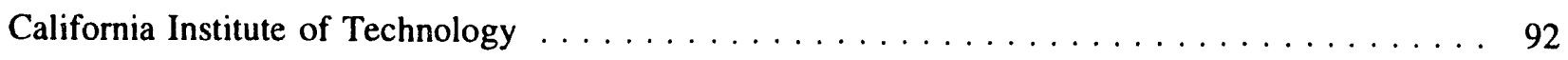

California State University, Northridge $\ldots \ldots \ldots \ldots \ldots \ldots \ldots \ldots \ldots$

Carnegie Mellon University $\ldots \ldots \ldots \ldots \ldots \ldots \ldots \ldots \ldots \ldots \ldots \ldots$

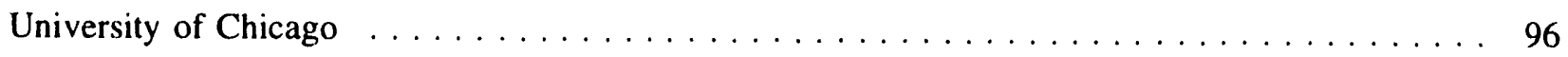

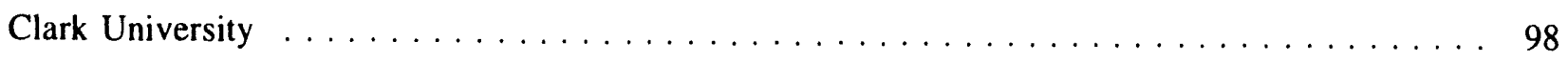

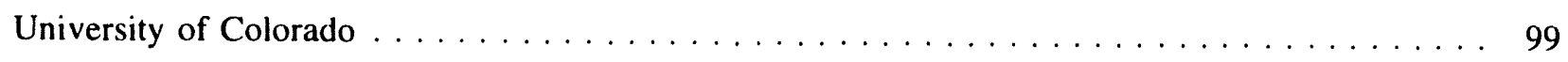

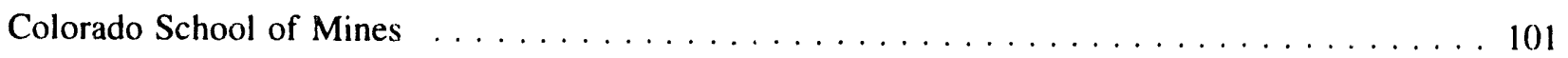




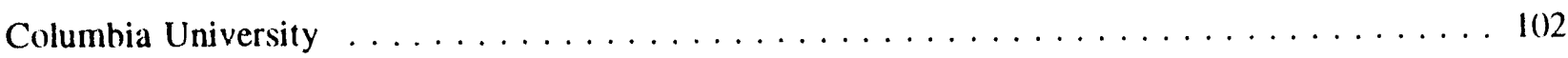

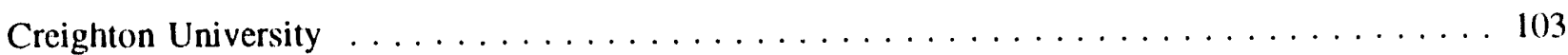

Denison University $\ldots \ldots \ldots \ldots \ldots \ldots \ldots \ldots \ldots \ldots \ldots \ldots \ldots \ldots \ldots$

Triangle Universities Nuclear Lahoratory at Duke $\ldots \ldots \ldots \ldots \ldots \ldots \ldots \ldots$

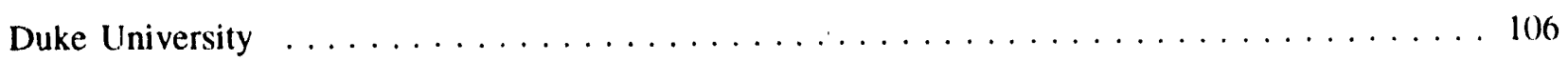

Florida State University $\ldots \ldots \ldots \ldots \ldots \ldots \ldots \ldots \ldots \ldots$

George Washington University $\ldots \ldots \ldots \ldots \ldots \ldots \ldots \ldots \ldots \ldots \ldots \ldots \ldots \ldots \ldots$

University of Georgia $\ldots \ldots \ldots \ldots \ldots \ldots \ldots \ldots \ldots \ldots \ldots \ldots \ldots \ldots \ldots$

Georgia Institute of Technology $\ldots \ldots \ldots \ldots \ldots \ldots \ldots \ldots \ldots \ldots \ldots \ldots \ldots$

Georgia State University $\ldots \ldots \ldots \ldots \ldots \ldots \ldots \ldots \ldots \ldots \ldots \ldots \ldots \ldots$

Hampton University $\ldots \ldots \ldots \ldots \ldots \ldots \ldots \ldots \ldots \ldots \ldots \ldots \ldots \ldots$

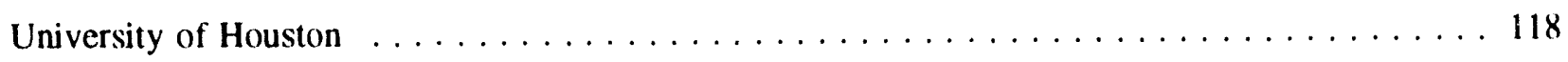

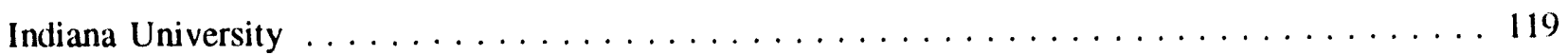

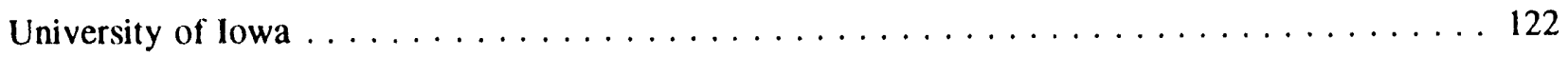

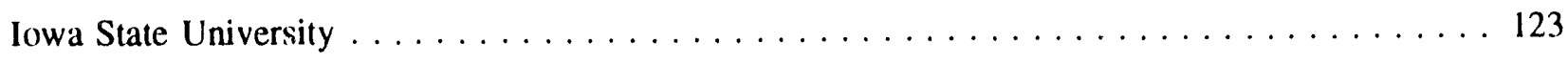

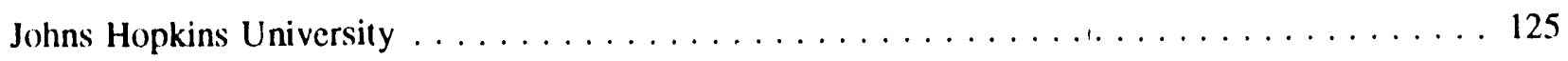

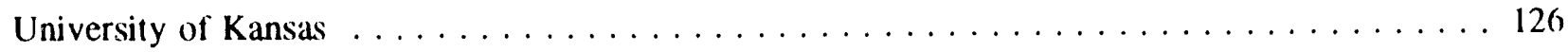

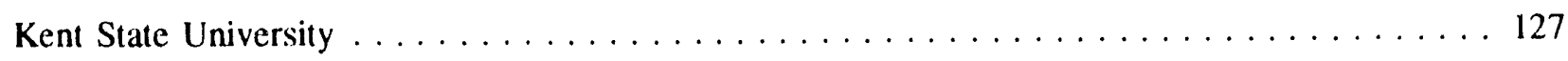

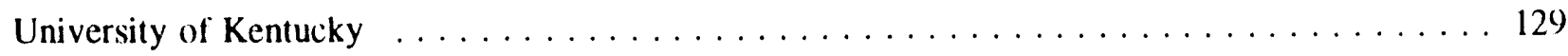

Louisiana State University $\ldots \ldots \ldots \ldots \ldots \ldots \ldots \ldots \ldots \ldots \ldots \ldots \ldots \ldots$ 130

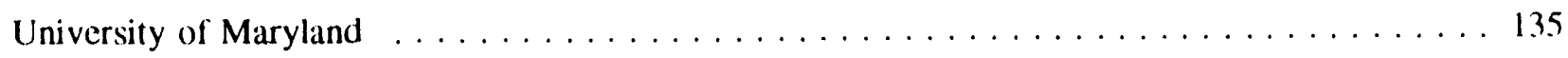

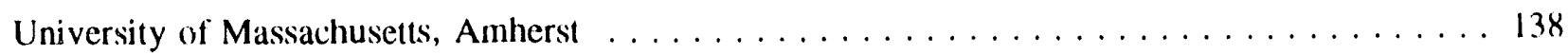

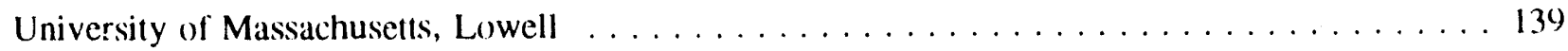

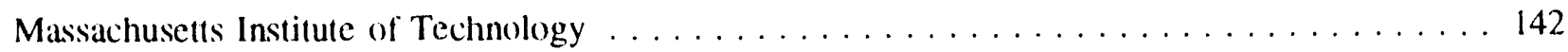

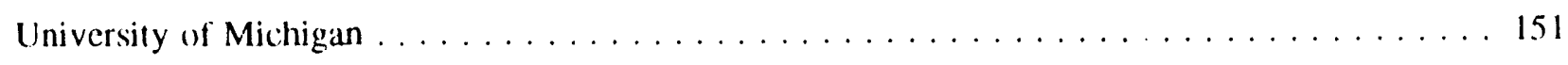

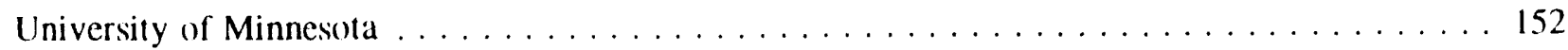

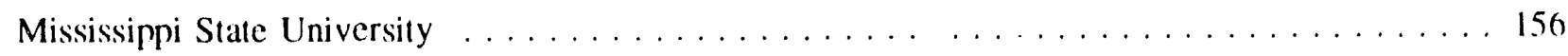

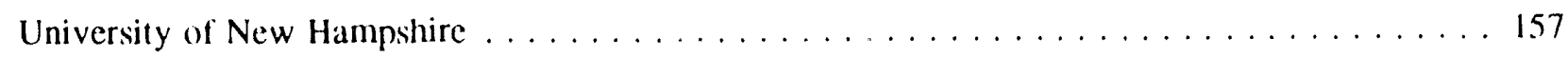

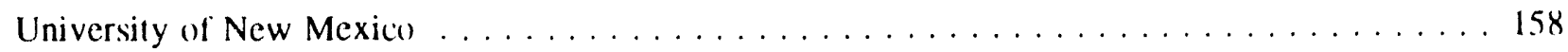

New Mexico State University . . . . . . . . . . . . . . . . . . . . 16()

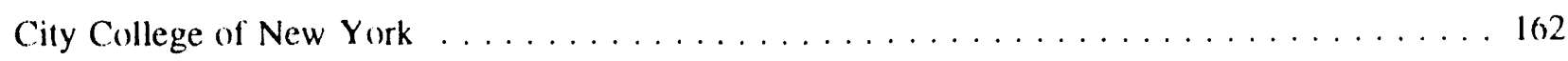




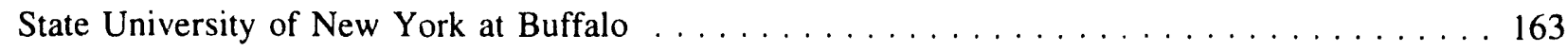

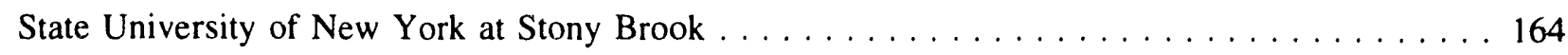

Norfolk State University . . . . . . . . . . . . . . . . . . . . . . . . . . . 167

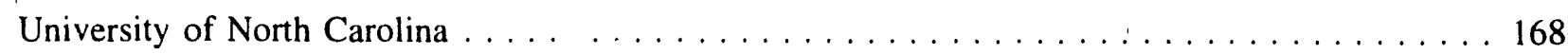

North Carolina State University . . . . . . . . . . . . . . . . . . . . . . . . . . . . . 169

Northwestern University $\ldots \ldots \ldots \ldots \ldots \ldots \ldots \ldots \ldots$

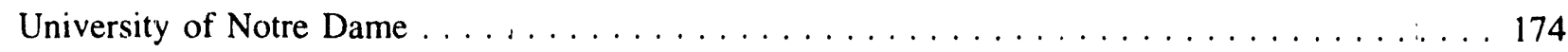

Ohio University . . . . . . . . . . . . . . . . . . . . . . . . . . . 175

University of Oregon $\ldots \ldots \ldots \ldots \ldots \ldots \ldots \ldots \ldots \ldots \ldots \ldots \ldots \ldots$

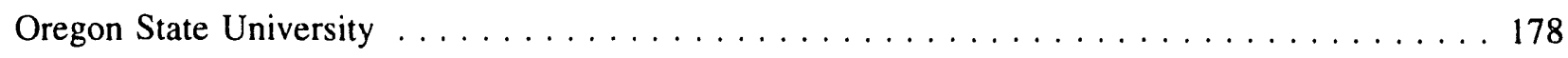

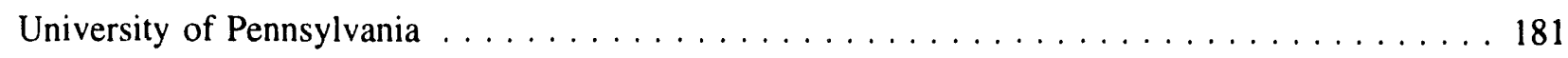

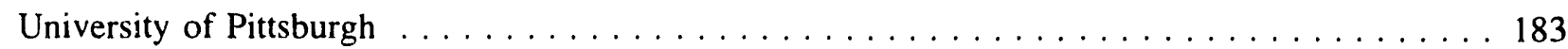

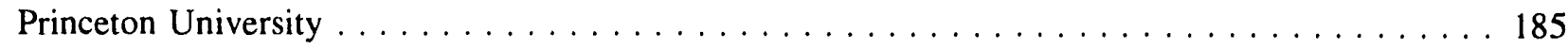

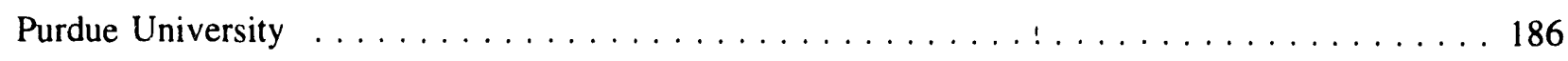

Rensselaer Polytechnic Institute . . . . . . . . . . . . . . . . . . . . . . . . . . . 189

Rice University $\ldots \ldots \ldots \ldots \ldots \ldots \ldots \ldots$

University of Richmond $\ldots \ldots \ldots \ldots \ldots \ldots \ldots \ldots \ldots \ldots \ldots$

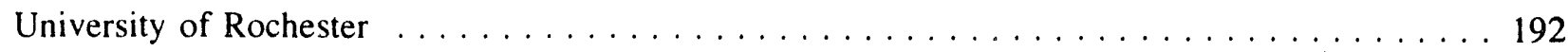

Rutgers University . . . . . . . . . . . . . . . . . . . . . . . . . . 194

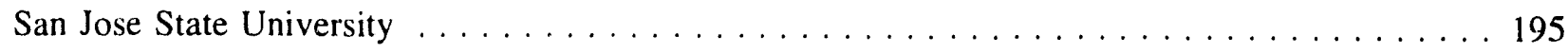

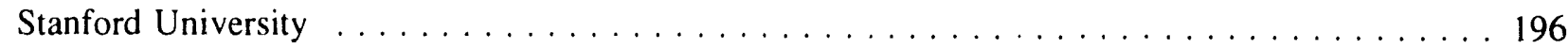

Syracuse University . . . . . . . . . . . . . . . . . . . . . . . 197

Temple University . . . . . . . . . . . . . . . . . . . . . . . . . 198

University of Tennessee . . . . . . . . . . . . . . . . . . . . . . . . . . 199

Middle Tennessee State University $\ldots \ldots \ldots \ldots \ldots \ldots \ldots$

Tennessee Technological University . . . . . . . . . . . . . . . . . . . . . 201

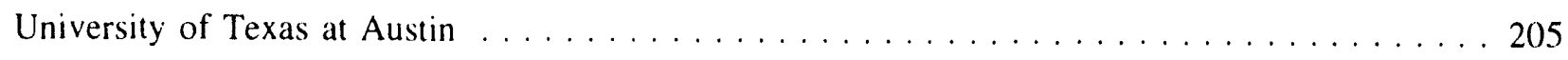

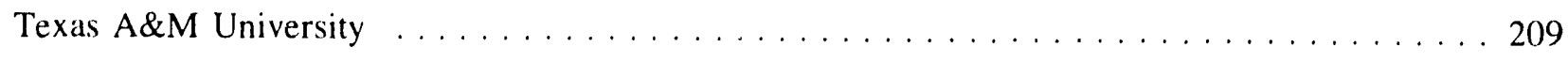

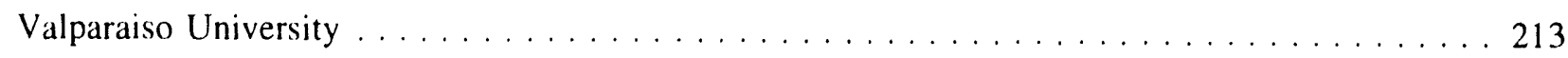

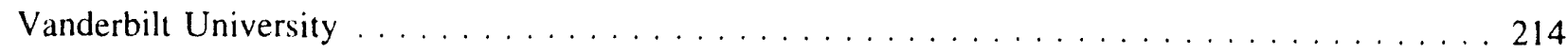

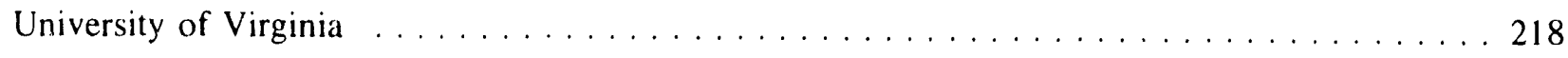


Virginia Polytechnic Institute and State University $\ldots \ldots \ldots \ldots \ldots \ldots \ldots$

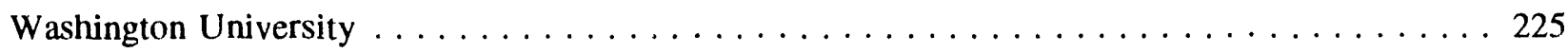

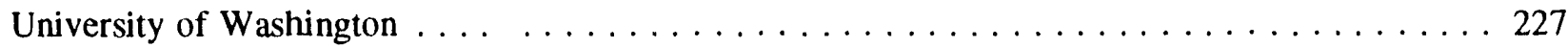

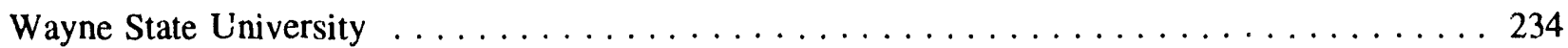

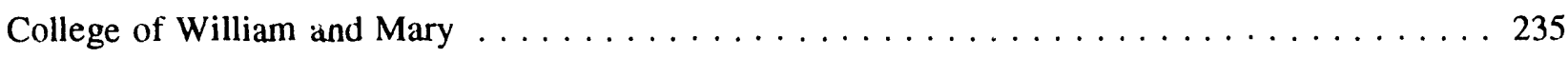

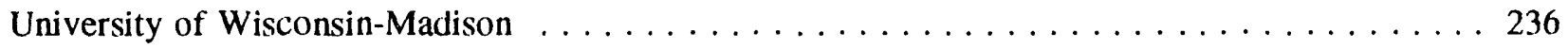

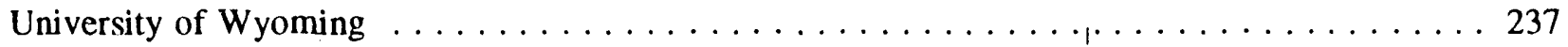

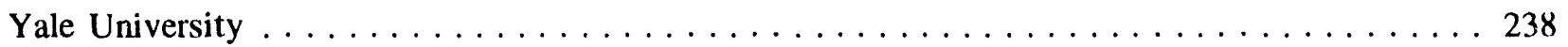

Glossary of Nuclear Physics Terminology $\ldots \ldots \ldots \ldots \ldots \ldots \ldots \ldots \ldots$

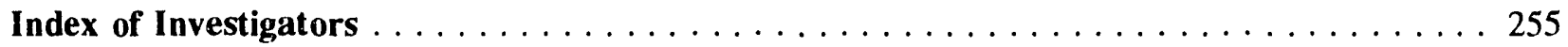


Research Progams at National Laboratories 


\section{ARGONNE NATIONAL LABORATORY}

Physics Division

Argonne, Illinois 60439

Title of Project: $\quad$ ATLAS Accelerator Facility

Persons in Charge: J. A. Nolen, J. P. Schiffer, W. F. Henning

Telephone:

(708) 252-4004

Scientific Staff: $\quad$ L. M. Bollinger, R. Harkewicz, R. C. Pardo, K. W. Shepard

FY 1992 Funding: $\quad \$ 4,032,000$

The Argonne Tandem Linac Accelerator System (ATLAS) facility, located in Argonne's Physics Division, is a heavy-ion accelerator system designed for precision studies in nuclear physics and other areas of science. The accelerator consists of a superconducting heavy-ion linear accelerator (linac) with two injectors, the original 8-MV tandem Van de Graaff with a negative ion source, and the new positive ion injector (PII). A small part of the linac was first operated in 1978, the world's first ion accelerator based on the phenomenon of superconductivity. Since that small beginning, the linac has been used steadily for research and has been gradually increased in size, capability, and reliability to its present status as a major facility capable of accelerating nuclei throughout the periodic table.

The new injector, PII, which was completed in 1992, consists of an electron-cyclotron resonance (ECR) ion source and a 12-MV superconducting injector linac. PII increases the available ion-beam intensity 100 -fold and will allow even the heaviest nuclei such as uranium to be accelerated to energies above the Coulomb barrier for nuclear reactions. This increased intensity and improved mass range, together with high duty cycle (continuous wave) and excellent time structure, make ATLAS heams highly desirable for a wide variety of investigations in basic nuclear science.

ATLAS is operated as a national collaborative research facility. It serves a large user community with more than 150 scientists and students participating in experiments every year from many institutions in the U.S. and from abroad. Running time for proposed experiments is allocated by a Program Advisory Committee. The experimental areas of ATLAS contain an array of major instruments that is available to all users: several large scattering chambers, a gamma-ray facility, an electron spectrometer, two magnetic spectrographs, an atomic-physics beam line, and general-purpose beam lines. In addition, a new experimental area containing two major instruments was recently completed. One of the instruments is the 8-meter long Fragment Mass Analyzer (FMA), which was completed in 1991. FMA has excellent mass-to-charge resolution and the capability of cleanly isolating rare isotopes produced in heavy-ion reactions. It will also be used in conjunction with the array of germanium and BGO gamma-ray detectors for detailed nuclear studies. The second apparatus in this new experimental area is the Argonne Positron-Electron Experiment, which is being commissioned in late 1992. A collaboration of eight universities and national laboratories will use this apparatus with the newly developed uranium and other heavy beams from ATLAS. Furthermore, since ATLAS beams are also ideally matched to the capabilities of the Gammasphere Detector currently under construction at LBL, plans are currently underway for a new beam line and room to house this device in the future. 
Physics Division

Argonne, Illinois 60439

Title of Project: $\quad$ Research at ATLAS

Persons in Charge: K. E. Rehm, J. P. Schiffer, W. F. Henning

Telephone: $\quad$ (708) $252-4004$

Scientific Staff: $\quad$ I. Ahmad, B. B. Back, R. R. Betts, M. P. Carpenter, C. N. Davids, M. Freer,

B. G. Glagola, R. Henry, R. V. F. Janssens, T. L. Khoo, W. Kutschera,

T. Lauritsen, Y. Liang, A. H. Wuosmaa

FY 1992 Funding: $\$ \$ 4,942,000$

Research at Argonne Tandem Linac Accelerator System (ATLAS), a unique superconducting accelerator capable of accelerating all ions up to uranium, ranges from studies of the high spin structure of nuclei to the search for exotic phenomena in collisions between very heavy nuclei. New experimental equipment, e.g., the Fragment Mass Analyzer (FMA) and Argonne Positron-Electron Experiment (APEX), a large magnetic solenoid for the detection of electron-positron pairs, allow new classes of experiments utilizing the new capabilities of the ATLAS accelerator. Unique applications include the detection of very rare nuclides by the method of accelerator mass spectrometry.

A new apparatus, the ATLAS Positron Experiment (APEX), has been constructed by an eightinstitution collaborat:on (ANL, Florida State, Michigan State, Princeton, Queen's, Rochester, Washington, Yale) to study features of positron production in collisions of very heavy ions. At Gesellschaft fur Schwerionenforschung (GSI) (Germany), positrons produced in extremely strong electric fields were studied, and unexpected narrow peaks were observed in the energy spectra. These peaks are not understood, and APEX, utilizing the new capabilities of ATLAS, provides an orderof-magnitude higher data-taking capability as well as superior resolution and event characterization compared to the previous experiments. First test experiments with U-beams from ATLAS started in late summer of 1992.

A major area of study is the structure of high spin nuclei and the response of nuclei to the large stresses induced by rapid rotation, which can only he attained from the collisions of heavy nuclei. Under these conditions, the properties and shapes of nuclei change until, at the highest rotational frequencies, the nuclei break apart (fission). Heavy-ion beams from ATLAS are used to produce nuclei which rotate very rapidly (up to about 100 units of angular momentum) and are highly excited. As the nuclei cool and slow down, they emit neutrons and gamma-rays, which are detected and yield information on the structure of the nuclei. The manner in which the nuclear shapes and the onset of fission depend on the spin, temperature, and the number of protons and neutrons is being mapped out. From this information, the stability of nuclei and their response to heating and rapid rotation are deduced. These experiments have been performed with a multi-detector array, constructed by Argonne and the University of Notre Dame, which is heavily used by researchers from many institutions in the U.S. and abroad. The new FMA with its excellent mass resolution is opcning new possibilities in these studies by tagging the $\gamma$-decay in coincidence with a nucleus of a specific mass. Argonne staff 
members are very much involved in the development and construction of Gammasphere, and ATLAS is ideally matched for the physics to he pursued with Gammasphere after its initial operation at LBL.

Another major area of research at ATLAS is the study of nuclei far from stability with FMA. In the fusion process hetween a heavy projectile and a target nucleus, new exotic nuclei are produced which do not exist in nature and decay rapidly by particle emission. Because these nuclei have extreme neutron-10-proton ratios, their properties contain valuable information about the nuclear force. The probahility for the production of these exotic nuclei is, however, quite small. To detect these nuclei with good efficiency, an ion-optical device consisting of magnets and electrostatic deflectors (FMA) has been installed at ATL.AS. It allows separation of these exotic nuclei according to their mass with excellent background suppression. The first experiments were carried out in 1991, and new isomeric and extremely neutron-deficient nuclei were found in the mass 150-180 region. Further improvements in the detection capabilities will allow new studies of very heavy nuclei $(A \approx 300)$.

A further component of research at ATLAS is the study of dynamics in the interaction of heavy nuclei at energies just sufficient to overcome their Coulomb repulsion. Here, the relative motion of the two nuclei is minimal, permitting studies of the interface between nuclear structure and the dynamics of nuclear collisions. The excellent timing and the wide variety of heavy-ion beams of ATLAS are ideally suited for the exploration of this area of nuclear physics. One class of experiments concentrates on how the structure of each nucleus affects the probability of two of them fusing into a single system. New techniques have been developed to measure the fusion probability at very low bombarding energies making use of the pulsed beam capabilities of ATLAS. Of considerable interest has heen the observation that even at very low bombarding energies, reactions involving large energy transfer ("deep-inelastic processes") occur. This result indicates that low energy nuclei emerge with large deformations from these collisions. Further experiments are planned to investigate this phenomenon, making use of inverse kinematics in reactions with heavy projectiles from ATLAS. 
Thtle of Project: $\quad$ Medium Energy Physics Research

Porsons in Charge: H. E. Jackson, J. P. Schiffer, W. F. Henning, H. Spinka, A. Yokosawa, L. E. Price

Tolophone: $\quad$ (708) 252-4004

Scientific Staff: $\quad$ K. Coulter, J. Freedman, D. Geesaman, R. J. Holt, S. Kaufiman, V. Papavassiliou, B. M. Poelker, D. Potterveld, B. Zeidman, D. P. Grosnick, D. Hill, D. Lopiano, M. Beddo, D. Underwood

FY 1992 Funding: $\quad \$ 2,817,000$

Medium energy research at ANL contributes to the understanding of the properties of nuclear matter by studying the interactions of nuclear constituents and the manner in which they are influenced hy the nuclear environment. The program emphasizes the study of processes in nuclei in which the constituents of the nucleon describe the basic physics, with the goal of incorporating the quark-gluon structure of the nucleon into a fundamental description of nuclear forces. Specific research topics include short range properties of nuclear forces, nuclear pion fields, and quark degrees of freedom in the nuclear medium. Because energetic leptons provide an accurate, well-understood probe of these phenomena, primary emphasis is placed on experiments involving electron and decp-inelastic muon scattering.

The medium energy physics program has a major presence in the research program at CEBAF. Staff memhers are actively involved in construction of the experimental facilities and have received approval of five research proposals for measurements when the heam hecomes availahle. Members of the group have assumed responsibility for the construction of a hroad-purpose Short Orbit Spectrometer (SOS) to be made generally available to the users at CEBAF. In FY 1991, a preliminary engineering design of SOS was completed; and in September 1991, first funding for component procurement and detailed engineering design was received from CEBAF.

In Fermilah experiment E665, deep-inelastic scattering of 5(0)-GeV muons has heen observed in coincidence with leading hadrons from a variety of nuclei. Noteworthy new results include ohservation of the ratios of xenon-10-deuterium cross sections down to $x$ values of $10^{-5}, 2$ orders-ofmagnitude lower in $\mathrm{x}$ than previous results. Nuclear shadowing saturates as $\mathrm{x}$ decreases below $\mathrm{x}-10^{-3}$ at the value of the ratio of cross sections observed in photoproduction experiments. The rates of twoforward jet events, attributed to photon-gluon fusion and gluon bremsstrahlung, agree with the predictions of QCD.

The tensor polarization of the recoil deuteron at very high momentum transfer is very sensitive to subnucleonic effects in nuclei, most notably meson-exchange and quark effects. A collaboration hetween the Argonne group and a Soviet group of physicists at Novosibirsk is engaged in a program of tensor polarization measurement: A polarized deuterium gas target intercepting the circulating heam of the VEPP-3 electron storage ring is employed, and the interactions of the deuterium gas with 
the circulating electrons are used to study polarization effects in elastic and inelastic scattering. To date, the ANL-Novosibirsk work represents the thickest polarized gas target operating in a storage ring.

Members of the program continue an active collaboration in the nuclear physics program at SLAC. During 1991, measurements of the photodisintegration of the deuteron were extended to $4.2 \mathrm{GeV}$ in experiment NE17, in which Argonne provides the leadership. Preliminary results provide strong evidence for the failure of the nucleon-meson description of the reaction. In the companion experiment NE18, a study of quasi-elastic scattering, measurements have been extended to high values of momentum transfer, never before accessible in (e,e'p) reactions, in a search for color transparency effects.

The study of the weak interaction at low energy is the other major component of the medium energy physics program. The main goals are to verify the implications of the Standard Model or to discover its inadequacies. An experimental search for neutrino oscillations with a 20-ton active neutrino detector positioned near the LAMPF heam stop is just heing completed. The final analysis provides limits on various modes of neutrino oscillations.

As the activities outlined previously at Fermilab, Novosibirsk, and SLAC conclude, work at CEBAF, particularly the commissioning of the SOS and initial measurements on approved proposals, will intensify. If approval by DESY of the HERMES proposal to study nucleon spin structure is received, the Argonne group will assume responsibility for particle identification in the HERMES proposal.

A lengthy program of measurements with polarized neutrons on polarized protons has been uncierway at Los Alamos (LAMPF) for a number of years. Many of the results of the elastic scattering and total cross section spin observable measurements have been published recently; the rest of the data is being analyzed. These experiments will have a major impact on the knowledge of the isospin-zero nucleonnucleon amplitudes and indirectly on the understanding of nucleon-nucleus scattering. A new program involving nucleon-nucleon spin measurements has recently begun at the Saturne accelerator in Saclay, France, in collaboration with physicists from Saclay, University of Geneva, Dubna, and University of California at Los Angeles. The goals are to measure quasi-elastic neutron-proton scattering spin observables at energies above $1,100 \mathrm{MeV}$ and to obtain additional information on energy-dependent structure observed in proton-proton scattering data near $2,100 \mathrm{MeV}$. These measurements will provide some of the first information on the isospin-zero nucleon-nucleon interaction at these energies. 


\section{ARGONNE NATIONAL LABORATORY}

Physics Division

Argonne, Illinois 60439

Title of Project: Nuclear Theory

Persons in Charge: T.-S.H. Lee, W. F. Henning

Telephone:

(708) $252-4004$

Scientific Staff: $\quad$ R. R. Chasman, F. Coester, H. Eshensen, S. Landowne, S. C. Pieper,

C. Roherts, R. B. Wiringa

Current Funding: $\$ 1,090,000$

The principal areas of the theory program are nuclear dynamics with subnucleonic degrees of freedom, nuclear forces and nuclear many-body problems, heavy-ion interactions, and nuclear structure. All 'research programs involve fundamental theoretical formulations of the problems and extensive confrontations with experimental data from major nuclear physics experimental facilities.

Starting with effective Lagrangians and quark models, theoretical approaches have been developed to describe nuclear dynamirs in the kinematic regions where the hadronic and electromagnetic productions of mesons and nucleon resonances are important. A systematic approach is heing developed to investigate the nonperturbative aspects of QCD, aiming at a fundamental understanding of nuclear dynamics in terms of quark-gluon degrees of freedom. The light-front relativistic formulation has been applied to investigate electromagnetic nucleon form factors, high energy photodisintegration of the deuteron, and the spin structure functions of three-nucleon systems.

Variational Monte Carlo methods have been developed to calculate nuclear properties from realistic two- and three-nucleon potentials. The predicted nuclear wavefunctions have been used to investigate various electroweak processes of astrophysical importance. Extensive many-body calculations are heing carried out to investigate the correlation effects on nuclear transparency and electron scattering from light nuclei.

A unified coupled-channels model has been developed to describe heavy-ion elastic scattering, inelastic scatteriag, nucleon transfer, and fusion processes in terms of a limited number of nuclear parameters. Our current interest is to study heavy-ion reactions induced by radioactive beams. A three-body model of : $\mathrm{Li}$ has been developed to successfully describe various nuclear reactions induced by heavy ions as well as pions. Models using effective two-body interactions to produce correlations in nuclear eigenstates have been used to interpret and predict various nuclear properties. The current focus is the investigation of superdeformation at both high and low spins. Our predictions of various superdeformed nuclei have led to the discovery of new regions of superdeformation at ATLAS. 
Engineering Physics Division

Argonne, Illinois 60439

Title of Project: $\quad$ Fundamental and Applied Neutron Physics and Nuclear Data

Person in Charge: $\quad$ A. B. Smith

Telephone:

(708) $972-6048$

Scientific Staff: $\quad$ P. Guenther, R. Lawson, J. Meadows, D. Smith

FY 1992 Funding: $\quad \$ 937,000$

The following is a summary statement of the Argonne neutron physics and nuclear data activities.

Energy-averaged neutron total cross sections are the only unambiguously calculable neutron-induced process and are essential for both fundamental and applied understanding. This activity has provided comprehensive total-cross-section information extending from a few tens of $\mathrm{keV}$ to $20 \mathrm{MeV}$, and spanning the mass range $A=6 \rightarrow 240$. These data remain a unique resource.

These endeavors have been the world's primary (and in many cases, only) source of fast-neutron scattering information, with results extending from a few $\mathrm{keV}$ to $100 \mathrm{keV}$ to $10+\mathrm{MeV}$ for a wide range of targets, extending over the mass range $A=6 \rightarrow 240$, and relevant to actinides, fission products, structural materials, and coolants found in fusion and fission energy systems.

An extensive program of fast-neutron capture studies, using both direct-detection and activation techniques, has been carried out in the $\mathrm{keV}$ to $\mathrm{MeV}$ range. The emphasis has been on fission-product and fertile materials. In some cases, these results remain the only available data.

Absolute and relative fission cross sections have been determined from a few $\mathrm{keV}$ to $10 \mathrm{MeV}$ for actinides in the range $230 \leq \mathrm{A} \leq 242$. The results form a standard for comparison throughout the world. An associated activity is precise standard determination and the development and exploitation of unique and precise flux-measurement devices (e.g., the "Black Detector").

The program has heen a major source of radioactivity information for dosimetry, radiation damage, materials assay, and waste management purposes. These results are in the context of precise differential values and standard-reference fields. ?

The program has enjoyed "world class" theoretical capability, particularly in the areas of compoundnucleus processes, the shell and optical models, reaction theory, and the dispersion phenomena. Such understanding and the associated models remain essential for the provision of data for applied purposes, and the work has made very substantive contributions to basic understanding achievable only with the neutron probe.

Properly, ti eie has heen notable success in transferring the experimental and theoretical results to the applications areas and to the private sector. The effort has been a major source of evaluated data for the national evaluated file system (ENDF). Recently, there have been extensive cooperative efforts with the private sector, particularly in the use of nuclear technologies for nondestructive interrogation (e.g., for explosive and drug detection and for nondestructive materials assay). 
A key factor in the success has been a unique mono-energetic neutron-source facility (the Fast Neutron Generator and associated equipment). Over nearly a quarter of a century, accelerator duty cycles of $\approx 81 \%$ have been achieved. At the same time, the safety and environmental record has been outstandingly good. 


\section{BROOKHAVEN NATIONAL LABORATORY}

Department of Physics

Upton, New York 11973

Title of Project: Relativistic Heavy-Ion Collider

Persons in Charge: S. Ozaki, N. P. Samios

Telephone:

(516) $282-5590$

FY 1992 Funding: $\quad \$ 49,350,000$ (LIC Funds)

RHIC is an ongoing project to build a versatile high energy heavy-ion colliding beam facility.

In RHIC, two beams of heavy ions will travel in opposite directions around a pair of superconducting magnet rings in a tunnel $3.8 \mathrm{~km}$ in circumference. The beams will collide at six different points around the accelerator, and each collision will have a combined energy of up to $100 \mathrm{GeV}$ for each of the protons and neutrons contained in the colliding nuclei $(100 \mathrm{GeV} / \mathrm{A})$. Collisions that combine heavy nuclei and extremely high energies are the key to creating a quark-gluon plasma. This cannot be done at existing high energy accelerator facilities.

The RHIC accelerator complex will include several existing BNL facilities. Heavy ions for RHIC will originate in Brookhaven's Tandem Van de Graaff, proceed into the Booster, and then into AGS. From there, the ions will be extracted in bunches and transferred to one of the two collider rings. Up to 57 bunches will be accepted in each ring. Then, with each ring filled, the ions will be accelerated in a matter of minutes to the top energy. At that energy, the ion beams will coast around the rings in stable orbits for hours. For experiments, at points where the beams cross, particles wili collide headon at the rate of tens of thousands of collisions per second. The tunnel configuration provides for six areas where the circulating beams cross and collisions take place. Four of these areas have the necessary enclosures and support buildings to accept the first phase of research detectors. Two areas will be left for a later phase of the development.

The traditional evolution of high energy accelerators has been to probe deeper and deeper into the structure of elementary particles. With beams of electrons or protons, the higher the energy of the beam, the more powerful is the "microscope," as scientists search for new particles of ever-smaller dimensions. RHIC will be the first high energy accelerator to take a different approach. By colliding large particles (heavy nuclei) at high energies, RHIC will create a microcosm of the hot, dense plasma of quarks and gluons from which particles, as we know them, condensed immediately after the Big Bang of the early universe. Thus, RHIC will be like a giant pressure cooker that will produce temperatures and pressures more extreme than exist now even at the centers of stars-creating new states of matter and opening the way to a new understanding of the relationship between the fundamental constituents of matter and the complex array of particles and nuclei that make up our universe.

In 1983, the Long Range Plan for Nuclear Science prepared by the scientific community for U.S. DOE and NSF cited RHIC as the highest priority new facility for the field. That recommendation was repeated in the 1989 Long Range Plan for Nuclear Science. The Presidential budget proposal for FY 1991 included funding to initiate RHIC construction. In FY 1991, the U.S. Congress appropriated 
the first construction funds for RHIC. After final review by DOE, the Federal agency funding the project, construction was formally hegun. RHIC will be constructed over a 7-year period, at a Total Estimated cost of $\$ 406,600,000$. About one quarter of the funding will he reserved for experimental detectors, which will be placed at the collider's collision points. The collider will be ready for experiments in 1997. 
Alternating Gradient Synchrotron Department

Upton, New York 11973

Title of Project: $\quad$ Tandem/AGS Heavy-Ion Accelerator Facility

Persons in Charge: D. Lowenstein, M. Schwartz.

Telephone: $\quad$ (516) $282-4611$

Scientific Staff: $\quad$ L. Ahrens, J. Alessi, D. Beavis, G. Bennell, M. Blaskiewicz, E. Bleser, M. Brennan, H. Brown, G. Bunce, A. Carroll, I. H. Chiang, B. Culwick, G. Danhy, C. Gardner, A. Hershcovitch, H. Hseuh, J. Kats, D. Lazarus, Y. Y. Lee, E. Lessard, A. Luccio, S. Mane, J. Niederer, P. Pile, K. Prelec, L. Ratner, T. Roser, M. Tanaka, P. Thieberger, W. vanAsselt, W. Weng, K. Woodle, M. Zarcone

FY 1992 Funding: $\quad \$ 8,850,(0)$

The Tandem/AGS Heavy-Ion Facility is a unique accelerator complex that provides beams for relativistic heavy-ion research in an energy range up to $14.6 \mathrm{GeV} / \mathrm{A}$. It is the only U.S. heavy-ion facility with center-of-mass energies well above the nucleon mass. The facility consists of three linked machines, the Tandem, Booster, and AGS. The Tandem injects heams of several MeV/nucleon into the Booster, which in turn accelerates these ions to energies of several hundred to a

thousand MeV/nucleon. Ions up to the mass of gold have been injected into AGS. There, the ions are accelerated to an energy of about $12 \mathrm{GeV} /$ nucleon and extracted to the AGS experimental areas for physics research. Since 1986, oxygen, silicon, and gold beams have been delivered to experiments. The completion of the Booster accelerator in 1991 permitted the accelerauon of the heaviest ions such as gold.

The heavy-ion research program at AGS simultaneously supports four large scale relativistic heavy-ion experiments and many smaller experiments. The goal of the rescarch is to study the properties of extremely hot and dense nuclear matter formed by collisions of heavy ions. Twenty-five experiments, including five of the major efforts, have already heen completed. Presently, ahout 374 researchers representing 61 institutions are involved in relativistic heavy-ion experiments that are either approved or running.

The construction of the Booster synchrotron for AGS has been completed. It now accelerates ions from the Tandem up to $A=200$ to sufficient energy that the capability of AGS has been extended to gold. The complex of Tandem-Booster-AGS provides a full range of heavy-ion heams for fixed-target experiments at AGS energies, and it will also constitute the complete injector system for RHIC (100 GeV/A for each of the two colliding beams). At present, feasibility studies are heing performed for the possible concurrent use of heavy-ion heams ( 0.4 to $1.5 \mathrm{GeV} / \mathrm{A})$ from the Booster for other (mainly radiohiological) appliçations. 
Title of Project: Relativistic Heavy-Ion Collider (RHIC) Research \& Development

Persons in Charge: S. Ozaki, M. Harrison, T. Ludlam, E. Willen

Telephone:

(516) $282-7753$

FY 1992 Funding: $\$ 7,300,000$ (funding for this and the following project)

The proposed RHIC will be a dedicated research facility capable of accelerating two beams of nuclei to energies of $100 \mathrm{GeV} / \mathrm{AMU}$ (kinetic energies about 100 times the rest mass energy) and colliding them head-on. In the aftermath of such collisions, scientists will be able to study nuclear matter under thermodynamic conditions more extreme than any so far observed, approximating the temperatures and densities that characterized the expanding universe moments after the Big Bang, before the primordial quarks and gluons condensed into the present forms of nuclear matter. Very high energy heavy-ion collisions are the only means of producing such conditions in the laboratory, and they offer a unique avenue for nuclear and particle physicists to test theories of the nuclear force at the most basic level.

RHIC will use the existing AGS, AGS Booster, and Tandem Van de Graaff accelerator complex at Brookhaven as an injector. Construction of the Collider hegan in January 1991, and the scheduled completion date is August 1997.

A research \& development (R\&D) program has been in progress since 1987 to support the construction of this facility and its detectors. This effort includes a study of accelerator physics problems that arise in preparing for construction of a heavy-ion collider, as well as the development of specific components of the collider system, such as superconducting accelerator magnets, the radio frequency acceleration and storage systems, beam injection techniques, heam position monitoring, and cryogenic and vacuum systems. In addition, the R\&D program includes a coordinated study by a large community of physicists to specify detector designs for the first-round research program at RHIC and to develop required detector technologies.

Presently, R\&D for the superconducting magnets is in its final stages. Prototype dinoles, quadrupoles, sextupoles, and correctors are being prepared for a final system test (full cell of the arc lattice) prior to delivery of the first industrialty fabricated magnets. For detectors, a generic R\&D phase has been concluded, and work now addresses the needs of specific detector designs that are being prepared for construction. These include the two large RHIC detectors, Solenoidal Tracker at RHIC, and PHENIX. 
Upton, New York 11973

Title of Project: Development of Physics Program for RHIC and Research \& Development for Detectors

Person in Charge: $\quad$ T. W. Ludlam

Telephone: $\quad$ (516) 282-7753

Scientific Staff: $\quad$ W. Guryn, E. Kistenev, Y. Makdisi, A. Stevens, T. Throwe, S. White, M. Ye

The RHIC research program focuses on the study of extended volumes of hadronic matter at extreme values of temperature and energy density in an experimental environment where the combined effects of event rate, total energy per collision, and final-state multiplicity far exceed conditions which have yet been encountered in nuclear or particle physics experiments. The project schedule calls for experiments to begin in FY 1997 with experiments in four intersection regions at the collider.

As of March 1993, two international collaborations of university and national laboratory scientists have been given scientific approval for experiments utilizing large detectors and have prepared conceptual design reports in preparation for detector construction. The Solenoidal Tracker at RHIC (STAR) detector is a cylindrical device based on a solenoid magnet and a large TPC for trackins and particle identification. The physics emphasis is on the measurement of hadron production over a large solid angle in the central rapidity range, with the ability to correlate global observables on an event-by-event basis in a search for signature of quark-gluon-plasma formation, and to use hard scattering of partons as a probe of the properties of high density nuclear matter.

The STAR TPC will be augmented by a three-layer silicon vertex tracker (SVT), utilizing the silicon drift technique, for improved momentum measurement and detection of secondary decay vertices. Although not part of the initial construction phase, SVT will be developed through its prototype phase as part of the RHIC research and development effort and constructed thereafter using other resources. The initial STAR construction phase will cost about $\$ 39$ million (FY $1992 \$$ ), of which $\$ 31.5$ million will come from RHIC project funds and the remainder from other resources within the collaboration. An electromagnetic calorimeter, for which outside funding is being sought, would be used to trigger on transverse energy and measure jet cross sections.

With this detector configuration, the major physics goals of STAR will be accomplished. A time-offlight system surrounding TPC for particle identification at higher momenta and external time projection chambers at forward angles, outside the magnet, to extend the rapidity coverage, are identified as potential upgrades.

The PHENIX Collaboration has designed a detector to explore the physics signals carried by leptons and photons. These are the so-called penetrating probes: electromagnetic particles whose characteristic spectra may reflect the thermodynamic conditions and particle states that prevail during the earliest stages of the formation of high density nuclear matter in RHIC collisions. Based on an axial magnetic field in the central rapidity region, with a forward muon arm, the PHENIX plan describes a detector system capable of measuring thermal and resonance spectra with electron pairs, 
muon pairs, and photons, as well as hadron production in the selected solid angle. The "basic" version of the detector is designed to address the key physics goals on day one, and its ultimate configuration will be matched to the available financial resources of the collaboration. Two electron/hadron spectrometer arms cover \pm 0.35 units of rapidity and a total of $180^{\circ}$ of azimuth. They include drift chambers for particle tracking, with particle identification provided by ring imaging Cerenkov counters, ionization loss measurement, time-of-flight, and electromagnetic calorimetry. The forward muon arm covers the ingular range from $10^{\circ}-35^{\circ}$, over the full azimuth.

More than half of the scientists and engineers that make up the PHENIX Collaboration are from foreign countries, and it is expected that a substantial portion of the funding resources for the construction of the detector will come from these sources. Like STAR, PHENIX will have a base construction budget of $\$ 31.5$ million (FY 1992) from the RHIC construction project. The contribution to the basic PHENIX detector from foreign collaborators is expected to be approximately $\$ 10-\$ 15$ million, from Japan, Russia, and China.

Two small detectors for RHIC have been given preliminary approval to prepare conceptual designs. These experiments, each with a total cost less than $\$ 5$ million, are designed to complement the large detectors by focusing on specific measurements, with apparatus that can be put in place relativery quickly and complete data-taking in about 1 year of operation.

PHOBOS is to consist of two spectrometer arms utilizing silicon pad and strip detectors and two small high field magnets. This detector will measure and identify very soft (low momentum) hadrons, whose production in RHIC collisions may have anomalous features related to the physics of a new phase of matter produced with relatively' large characteristic scales of time and distance. Another of the smaller detectors, the Forward Angle and Midrapidity Hadron Spectrometer, will provide RHIC. with the capability for measuring inclusive momentum spectra of identified charged hadrons (pions, kaons, protons, and their antiparticles) over a range of rapidity $₫ 4$ units) that extends well beyond the central rapidity region explored by the large detectors. This spectrometer should make the connection between particle production in the central region where the net density of baryons is expected to be low (e.g., nearly equal numbers of protons and antiprotons) and the "baryon-rich" material produced at small angles relative to the collision axis.

Other proposals currently under discussion include ideas for measuring elastic scattering at RHIC, examining particle production via the interaction of very intense electric and magnetic fields accompanying the charged ions colliding at high energy, and a continuation at RHIC of the search for "strangelets"-massive baryons whose makeup includes large numbers of strange quarks-that was begun at AGS. 
Upton, New York 1:973

Title of Project: Relativistic Heavy-Ion Experiment at AGS-E866

Persons in Charge: C. Chasman, P. D. Bond

Telephone:

(516) $282-3990$

Scientific Staff: $\quad$ D. Bèavis, Z. Chen, Y. Y. Chu, J. B. Cumming, R. Debbe, M. Gonin, S. Gushue, O. Hansen, S. Hayashi, M. J. LeVine, B. Moskowitz, J. Olness, L. P. Remsberg, M. J. Tannenbaum, J. H. van Dijk, F. Videbaek, H. E. Wegner

FY 1992 Funding: $\$ 3,060_{i} 000$ (funding for this and the following two projects)

It is generally accepted that nucleons, the building blocks of nuclei, are themselves composite particles rather than elementary particles. A nucleon is composed of quarks, three in each nucleon, whereas a meson is comprised of two quarks. An interesting feature of quarks is that a single quark has never been observed by itself. The reason quarks are confined is not fully understood, and whether quarks can somehow be let loose or have more than three joined together is therefore a question of primary importance in physics. The early universe, shortly after the Big Bang, is believed to have passed through a stage in which the quarks were free (deconfined). One of the best methods to address the confinement problem is expected to be through observation of collisions of very energetic large nuclei. E866, an experiment involving about 20 scientists from Brookhaven and 35 nuclear scientists from other institutions, is an attempt to look at such behavior. This experiment is similar in philosophy to predecessor experiments E802 and E859, but it utilizes the gold beam-the most massive beam ever accelerated to AGS energies. These high energy gold particles are collided with gold nuclei. The residuals of the ensuing explosion, baryons and mesons, are analyzed using a magnetic spectrometer . that tracks some of the particles, employing time-of-flight and Cerenkov counters for particle identification. The total number of emitted charged particles and the total energy of the particles which go forward are also measured in order to characterize the severity of the nuclear collision.

The experimental signature for quark deconfinement produced by such energetic heavy nuclei is not certain, but there has been much well-reasoned speculation. The experiment therefore aims at comparing the results obtained with the gold beam to those obtained with lighter projectiles such as proton, oxygen, and silicon beams in E802 and E859, in order to delineate the specific effects of high energy and baryon density anticipated in these reactions. The experiment is also sensitive to some predicted characteristics of quark deconfinement, such as the yield of strange particles and antiparticles, and to the size of the source emitting these particles. 


\section{BROOKHAVEN NATIONAL LABORATORY}

Department of Physics

Upton, New York 11973

Title of Project: Relativistic Heavy-Ion Collisions at AGS--E877

Persons in Charge: T. Ludlam, P. Baun-Munzinger (SUNY Stony Brook)

Telephone: $\quad$ (516) 282-7753

Scientific Staff: $\quad$ D. Lissauer, S. McCorkle, E. O'Brien, V. Polychronakis, H. Takai, T. Throwe, C. Woody

The goal of experiment E877 is to examine the properties of particle production and energy deposit in collisions of heavy nuclei (e.g., gold nuclei), accelerated to approximately $10 \mathrm{GeV} / \mathrm{amu}$ by the Tandem-Booster-AGS accelerator complex, with heavy nuclear targets. The experiment utilizes the $4 \pi$-calorimetry, multiplicity counters, and forward spectrometer of the earlier experiment E814. With some modifications to this detector system, experiment E877 is designed to measure the distributions in transverse energy and multiplicity, and spectra of baryons and mesons with good coverage for low and intermediate values of transverse momenta, and rapidities greater than 1.5 in the laboratory frame. The E814 calorimeters and appropriately upgraded multiplicity counters provide full event characterization.

For E877, the magnet geometry in the detector is rearranged to allow greater coverage in transverse momentum and rapidity. A high granularity, high resolution time-of-flight wall is to be added to the E814 forward spectrometer, at a distance of about 12 meters from the target. This will provide, in conjunction with the tracking chambers, complete particle identification for nucleons, antinucleons, deuterons, and heavier nuclear fragments. This set-up will also allow separation of kaons and pions up to momenta of about $4 \mathrm{GeV} / \mathrm{c}$.

The first data with gold beams were obtained during the spring of 1992. The detector upgrades are expected to be fully in place by the end of 1993 . 
Department of Physics

Upton, New York 11973

Title of Project: Search for New Phenomena in Heavy-Ion Collisions-E810

Persons in Charge: K. J. Foley, P. D. Bond

Telephone: $\quad$ (5:5) 282-3943

Scientific Staff: $\quad$ A. Etkin, R. W. Hackenburg, R. S. Longacre, W. A. Love, E. D. Platner, A. C. Saulys (BNL); S. J. Lindenbaum (BNL/CCNY)

This experiment is a search for new phenomena in heavy-ion collisions at AGS and is a collaboration of 24 scientists from 3 institutions. The method chosen is to measure the angles and momenta of a major fraction of the charged particles from each collision; the acceptance is virtually 100 percent for particles produced in the forward hemisphere in the nucleon-nucleon center-of-mass. The enormous amount of information available for individual events can be used in a very sensitive search for new effects like the formation of a quark-gluon plasma. For example, anomalous antiproton and antilambda production have been suggested as possible signals, and one can take events containing such particles and examine them on a one-by-one basis for other unusual characteristics in multiplicity, momentum distribution, particle content, etc. Tius approach will reduce the background from "normal" cvents and greatly enhance the sensitivity to new phenomena.

The technizue chosen is a custom-designed TPC, a device known for its ability ic handle complex events. As part of the design, a custom hybrid readout chip has been developed to reduce the cost and enhance reliability. Several successful runs have taken place with the results reported in conferences and in publications. The production rate of $K^{\circ:} s$ and $\Lambda^{\prime} s$ is higher than predicted by simple models, as is the daia for charyed kaons, although more complex models including secondary interactions do fit much of the data. Analysis is proceeding to study other phenomena. 


\section{BROOKHAVEN NATIONAL LABORATORY}

Department of Physics

Upton, New York 11973

Title of Project: $\quad$ Nuclear Structure Investigations with Medium Energy Hadronic Probes

Persons in Charge: $\quad$ R. E. Chrien, P. D. Bond

Telephone:

(516) 282-3903

Scientific Staff: $\quad$ S. Bart, M. May, R. Sawafta, R. J. Sutter

FY 1992 Funding: $\$ 1,060,000$

Meson beams are used to investigate fundamental properties of nucleons and how they interact within the nucleus. These fundamental properties of nucleons are explored by use of particle heams of a variety and energy not available at other nuclear physics facilities.

Detailed information on the short range features of the nuclear interaction is provided by the AGS beam probes with wavelengths intermediate between the dimensions of a nucleon and the dimensions of a nucleus. The use of probes containing the quantum property of strangeness allows the investigation of the flavor properties of nuclear systems. For example, the study of nuclei with strangeness, or hypernuclei, and a comparison of nuclear and hypernuclear systems provides special insights into nuclear forces and nuclear structure.

The provisions of intense beams of pions and kaons at AGS allow the study of a wide spectrum of nuclear properties. The scattering of $\mathrm{K}^{+}$mesons by nuclei has created a broad interest in the properties of hadrons in a nuclear environment. The studies of the nuclear density dependence of $\mathrm{K}^{+}$total, elastic, and inelastic cross sections have suggested significant alteration in hadronic properties, such as mass and size, when the hadron is imbedded in the nuclear medium.

Various searches for quark conglomerates that do not exist as conventional nucleons are now underway at AGS. One class of experiment, such as experiment 886, searches for hits of strange matter termed "strangelets," which are characterized hy anomalously low charge-to-mass ratios. Others, s'ich as experiments 813 and 836 , are searches for the $\mathrm{H}$ dibaryon, an assembly of two-strange, two-up, and two-down quarks.

The usefulness of high energy pion beams for creating systems such as the $\eta$ meson, with the usual quark wavefunctions, is currently heing explored. The rare decays of the $\eta$ are useful in tests of the Standard Model. 
Title of Project: $\quad$ Laser Electron Gamma Source

Persons in Charge: A. M. Sandorfi, P. D. Bond

Telephone:

(516) 282-7951

Scientific Staff: $\quad$ O. C. Kistner, C. E. Thorn

S. Hoblit, M. Khandaker, D. Rebreyend, A. Tam

FY 1992 Funding: $\quad \$ 1,040,000$

Intense high energy gamma-ray beams, presently extending up to $360 \mathrm{MeV}$, are produced at Laser Electron Gamma Source (LEGS) by backscattering laser light from $2.5 \mathrm{GeV}$ electrons circulating in the $\mathrm{x}$ ray storage ring of the National Synchrotron Light Source at BNL. These gamma-rays are very highly polarized (typically $>90$ percent), monochromatic (with $\sim 5.5 \mathrm{MeV}$ resolution), and are accompanied by very low background levels (limited only by the cosmic ray flux). LEGS provides the only source of medium energy polarized photons in the country and is being used to study specific fundamental properties of nucleons and nuclei that can only be addressed with the polarization degree of freedom.

LEGS has been operating since the beginning of 1990, and so far six experiments have been completed. In experiments L1 and L3, the beam-polarization observables in deuteron photodisintegration were measured with high precision. Here, the difference of cross sections for orthogonal states of linear polarization is particularly sensitive to the short range behavior of the nuclear tensor force and provides important constraints on this interaction. In experiments L2 and L5, the polarization observables in neutral pion photoproduction from the proton have provided a crucial test for models that attempt to deduce the deformation of the nucleon, a test in which, so far, all models have failed. The polarization asymmetry in $p-p$ and $p-n$ final states, measured in experiment L4 from the photodisintegration of ${ }^{3} \mathrm{He}$, is quite sensitive to hypothesized nuclear three-body forces. Finally, the nucleon-delta interaction was studied in experiment L6 by detecting the decay of deuterium into $\mathrm{N}-\mathrm{N}$ pion final states following polarized photon absorption.

New measurements are underway that will provide tighter constraints on nucleon deformation. Experiment L7 will provide a new high quality multipole decomposition of the pion production amplitudes, and experiment L8 will focus on elastic photon scattering, a channel in which the polarization can be used to directly cancel backgrounds. Future experiments will measure the polarizabilities of the proton and the pion. These will provide sensitive tests of chiral-perturbationand quark-models of the internal structure of these hadrons. 
Title of Projoct: $\quad$ Nuclear Structure Studies

Persons in Charge: R. F. Casten, P. D. Bond

Telephone: $\quad$ (516) 282-3979

Sciontific Staff: $\quad$ R. L. Gill, N. V. Zamfir, E. K. Warburton

FY 1992 Funding: $\quad \$ 681,000$

This research centers on nuclear structure studies, with emphasis on nuclear symmetries and the role of the proton-neutron $(p-n)$ interaction in determining the evolution of collective properties of nuclei with $\mathrm{N}, \mathrm{Z}$, and $\mathrm{A}$. The research effort is mainly experimental, although there is considerable theoretical analysis as well.

Studies of unknown or nearly unknown nuclei provide key tests of the predictive power of nuclear models, can disclose new examples of fundamental symmetries, and offer sensitive probes of $p-n$ interaction effects, because such nuclei contain combinations of proton and neutron orbits otherwise inaccessible. $\gamma$-ray spectroscopy and magnetic moment measurements are carried out with the on-line isotope separator, the Tri Ring Intersecting Storage Accelerators in Nippon (TRISTAN), which has access to the widest array of neutron-rich nuclei available anywhere. These data also elucidate astrophysical nucleosynthesis, because the r-process path passes through nuclei accessible at TRISTAN. The Fast Electronic Scintillation Timing (FEST) technique allows the measurement of picosecond lifetimes and absolute transition rates heretofore impossible for neutron-rich nuclei so far off stability.

Thorough data near stability are complementary: They distinguish different models and symmetry frameworks. Complete spectroscopy is achieved with the nonselective $(n, \gamma)$ reaction in the Average Resonance Capture (ARC) mode using 2-keV and 24-keV neutron beams from the BNL High Flux Beam Reactor to access all nuclear states in certain energy and spin regimes. Collahorative work at the Institute Laue-Langevin is normally also an integral element of this program.

Recent work studied the linearization properties of valence correlation schemes such as $\mathrm{N}_{p} \mathrm{~N}_{n}$ and the P-factor, the phenomenology and microscopic basis for identical bands, energy staggering as a signature of shapes and symmetries, new roles for effective charges in valence models, intruder states, E1 transitions, and large basis shell model calculations. Finally, new initiatives involving exploitation of Radioactive Nuclear Beams to probe nuclei much further from stability are being actively pursued. 


\section{BROOKHAVEN NATIONAL LABORATORY}

Department of Chemistry

Upton, New York 11973

Title of Project: $\quad$ Gallium Solar Neutrino Experiment, GALLEX

Persons in Charge: R. L. Hahn, N. Sutin

Telephone:

(516) $282-4337$

Scientific Staff: J. K. Rowley, F. X. Hartmann, J. Weneser

FY 1992 Funding: $\quad \$ 737,000$

The aim of this research is to gain an understanding of the "solar neutrino problem," namely that the well-known chlorine and Kamiokande detectors measure fluxes of neutrinos from the sun that are less than one half of the theoretical values. Current explanations of these discrepancies require either that neutrinos are not produced in the sun's interior at the expected intensity or that they "disappear" en route to Earth. Either stellar physics is incomplete, or new neutrino properties, such as finite mass, must be invoked.

A new neutrino detector, using gallium as the active ingredient, was developed at BNL. Then, the Gallium Experiment (GALLEX), a consortium of 10 institutions located mainly in Europe, with BNL as the sole American participant, constructed the detector at the Gran Sasso Underground Laboratory in Italy.

GALLEX uses 30 tons of gallium in a 50,000-liter solution of gallium chloride and hydrochloric acid. Capture of a neutrino by ${ }^{71} \mathrm{Ga}$ produces radioactive ${ }^{71} \mathrm{Ge}$, which is separated from the target by ultrasensitive chemistry and counted via its $x$ rays and Auger electrons.

The theoretical production rate of ${ }^{71} \mathrm{Ge}$ in the gallium is 1.2 atoms per day. Of the existing neutrino detectors, gallium, with a $233-\mathrm{keV}$ threshold for neutrino capture, is the only one capable of detecting the abundant low energy neutrinos from the primary proton-proton ("pp") fusion reaction in the sun.

GALLEX hegan "looking at" the sun in May 1991. By April 1992, data from 14 solar exposures provided the first definite evidence of ${ }^{71} \mathrm{Ge}$ production in gallium: Both the characteristic energy spectrum and half-life of the nuclide were observed. The results were published in Physics Letters $B$ in July 1992.

The ${ }^{71} \mathrm{Ge}$ production rate measured by GALLEX was $(63 \pm 16)$ percent of the theoretical prediction. This value was sufficiently high to allow GALLEX to claim the first direct observation of the solar $\mathrm{pp}$ reaction. All previous evidence for the pp reaction was indirect, such as measurement of the solar !uminosity.

With its present large statistical uncertainty, the GALLEX result does not provide the long-awaited solution to the solar neutrino puzzle. But, additional years of data-taking by GALLEX should reduce the statistical errors by a factor of 2, allowing us to choose between the alternative explanations hased either on revisions in the solar model or on new neutrino physics. 
National Nuclear Data Center

Department of Nuclear Energy

Upton, New York 11973

Title of Project: $\quad$ Reference Nuclear Data for Energy Research

Persons in Charge: C. L. Dunford, M. R. Bhat

Telephone:

(516) $2 \times 2-2901$

Scientific Staff: $\quad$ T. W. Burrows, R. R. Kinsey, W. Kropp, V. McLanc, L. Peker,

S. Ramavataram, J. Tuli

FY 1992 Funding: $\quad \$ 2,658,()()()$

DOE hasic and applied research programs are facilitated when nuclear data are collected, compared, evaluated, and distributed for use. The National Nuclear Data Center (NNDC) contributes 10 and is the principal coordinator of efforts to provide low energy nuclear physics databases to the scientific community. The Cross Section Evaluation Working Group (CSEWG) and the U.S. Nuclear Data Network (USNDN) are interlaboratory groups coordinated by NNDC. NNDC is also the primary contact point for U.S. participation in international collaborative activities in the compilation and evaluation of nuclear data.

CSEWG completed its 26th year of activity in 1992. Emphasis in the past few years has heen on the completion and testing of Evaluated Nuclear Data File/B-VI, a reference file containing evaluated experimental and theoretical results for applied research. The tile has heen distributed both nationally and internationally for testing and use.

With the help of USNDN and international collahorators, NNDC produces the refereed journal Nuclear Data Sheets. Nine issues a year are devoted to evaluated nuclear structure data and the remaining three to the publication of recent references. In addition to coordinating the U.S. evaluation activity, NNDC contrihutes to the evaluation task.

For approximately 6 years, NNDC has offered on-line access to its nuclear databases. This service is available on NNDC's VAX-8820 computer via Energy Sciences network (ESnet), INTERNET, or over telephone lines. In recent years, video interfaces have heen developed to make their use simpler. Graphical as well as tahular output are availahle from several numeric datahases. New users can sign up for service during a special limited access session. In the past year, approximately 2,200 retrievals per month were done hy users. 
Department of Physics

Upton, New York 11973

Title of Project: $\quad$ Nuclear Theory

Persons in Charge: C. B. Dover, P. D. Bond

Telephone:

(516) $282-3791$

Scientific Staff: $\quad$ A. J. Baltz, S. Gavin, S. H. Kahana, D. J. Millener, Y. Pang, T. J. Schlagel, J. Weneser

FY 1992 Funding: $\quad \$ 1,205,000$

The principal focus of the Nuclear Theory Group is relativistic heavy-ion physics, although topics in hypernuclear structure, solar neutrino physics, and areas at the interface hetween elementary particle physics and nuclear physics are also vigorously pursued. Much of this effort is characterized hy close attention to experimental results and active ties with experimental programs at Brookhaven and elsewhere.

As a method of directly confronting the copious experimental data on relativistic heavy-ion interactions, a relativistic cascade code, Average Resonance Capture (ARC), has heen developed hy the group. When applied to $\mathrm{Si}+\mathrm{Au}$ (at the AGS energy of $14.6 \mathrm{GeV} /$ nucleon) collisions, ARC quantitatively describes most of the data from AGS experiments E8(2), E810), and E814. Other problems such as antiproton and composite particle production are under investigation. The ARC code is heing extended to the parton level, to study the dynamics of higher energy relativistic heavy-ion collisions at RHIC.

We have undertaken an examination of electromagnetic processes that influence the RHIC heam lifetime. Calculations of the hound-electron-positron production appear now to he only moderately greater than the perturbation-theory result.

Our efforts in intermediate energy physics include investigations of particular relevance to future experiments at AGS. Examples are (1) processes hy which double hypernuclei could he formed and detected, (2) two-nucleon mechahisms for the production of dibaryons, and (3) studies of spin phenomena in hypernuclei. The physics of nucleon-antinucleon processes has been a continuing interest, and recent work has been focused on the dynamics of tucleon-antinucleon annihilation in a microscopic quark model and the production of exotic mesons.

We have pursued the study of effective four-fermion models, which generate gauge hosons as composites. This leads to very interesting predictions of hoth the top quark and Higgs hoson mass.

Members of this group are closely involved with the solar neutrino problem: One is a member of the Gallium Experiment Collaboration, and another a part of the BOREXINO group.

The long term direction of our research program will he primarily focused on relativistic heavy-ion physics. 


\section{CONTINUOUS ELECTRON BEAM ACCELERATOR}

F A C I LIT Y

12000 Jefferson Avenue

Newport News, Virginia 23606

Title of Project: Construction of the Continuous Electron Beam Accelerator Facility

Persons in Charge: H. A. Grunder, B. Hartline

Telephone:

(804) $249-7552$

FY 1992 Funding: $\quad \$ 48,300,000$

CEBAF is being constructed for use as a basic research laboratory in nuclear physics. Its mission is to obtain precise information on the structure of strongly interacting matter, including the role of quarks and gluons within nucleons and nuclei.

CEBAF is based on a 4-GeV superconducting recirculating electron linear accelerator that will produce a 100 percent duty factor, 0.5 to $4.0 \mathrm{GeV}, 200$-microampere beam for simultaneous delivery to three experimental halls. Enclosed in a racetrack-shaped tuninel 1,300 meters in circumference, this accelerator will cycle the beam through a segment of linear accelerator (linac) in each straightway. Magnetic arcs transfer the beam between the north and south linacs. The beam gains up to $800 \mathrm{MeV}$ in each of the five complete circuits. The linacs have a total of 338 five-cell niobium radio-frequency accelerating cavities that are linked in pairs, placed in insulating cryostats or cryomodules, and cooled to $2 \mathrm{~K}$ by immersion in liquid helium. At this temperature, the cavities are superconducting. The use of superconducting radio-frequency cavities enables the delivery of electron beams having a 100 percent duty factor.

Construction of the facility began in February 1987 and is scheduled for completion in 1995 with Federal funding totaling $\$ 551.4$ million, including $\$ 36.5$ million in additional capital equipment funds. Experiments are scheduled to begin in 1994 with delivery of an $800-\mathrm{MeV}$ electron beam to one experimental hall. Independently tunable heams with energy up to $4 \mathrm{GeV}$ will be available simultaneously to all halls in late 1994.

As of August 1, 1992, the project is 75 percent complete and 83 percent obligated. The project remains on cost and on schedule with civil construction scheduled for completion in Novemher 1992. The accelerator installation is progressing well, and key contracts for major experimental equipment components are placed for all three halls. 


\section{CONTINUOUS ELECTRON BEAM ACCELERATOR \\ FACILITY}

12000 Jefferson Avenue

Newport News, Virginia 23606

Title of Project: $\quad$ CEBAF Research \& Development, Commissioning of the Accelerator and Administrative Support

Person in Charge: $\quad$ C. W. Leemann

Telephone: $\quad$ (804) 249-7554

Scientific Staff: J. F. Benesch, J. J. Bisognano, J. R. Boyce, I. E. Campisi, Y. C. Chao,

L. R. Doolittle, D. R. Douglas, H. F. Dylla, L. H. Harwood, J. A. Jackson,

R. Kazimi, J. B. Kewisch, P. K. Kloeppel, P. L. Kneisel, G. A. Krafft, R. Li,

H. Liu, N. Merminga, G. R. Myneni, G. R. Neil, D. V. Neuffer,

V. Nguyen-Tuong, H. L. Phillips, E. J. Price, C. E. Reece, R. Rossmanith,

S. Simrock, C. K. Sinclair, M. G. Tiefenback, B. C. Yunn

FY 1992 Funding: $\quad \$ 23,333,000$ (funding for this and the following project)

\section{Accelerator Performance Parameters}

Research at CEBAF is currently undertaken in support of the project and its evolving nuclear physics program. For the CEBAF accelerator, this means support for the design, fabrication, installation, and testing of components and assemblies to assure that construction milestones are realized on schedule, within cost, and with accelerator parameters maintained. The beam parameter objectives are:

- $4 \mathrm{GeV}, 200$ microamperes, continuous wave (cw).

- Emittance of $2 \times 10^{-9} \mathrm{~m}$.

- Energy spread of $2.5 \times 10^{-5}$.

- Simultaneous heam delivery to three experimental halls with independently adjustable energy and current.

\section{Accelerator Design}

The heam parameter objectives have led to the CEBAF accelerator design concept:

- cw for high heam quality.

- Power-efficient superconducting radio frequency (SRF) technology.

- Five-pass beam recirculation for cost optimization and multi-energy operation. 
Key design features include:

- 1 RF power system per cavity to control microphonics and provide operational flexibility.

- $2 \mathrm{~K}$ cost-optimizing operation.

- Isochronous, achromatic beam recirculation arcs.

- 2,264 magnets and strong focusing optics to minimize synchrotron radiation.

- A 70,000-channel control system.

\section{Accelerator Subsystems Scope and Status}

The CEBAF accelerator requires a total of 42 cryomodules containing 338 SRF cavities. The cavities are clean-room assembled into operating pairs-four pairs for each cryomodule-and then tested. In tests to date, the measured average gradient of the cavities is $9.0 \mathrm{MV} / \mathrm{m}$, well above the design specification of $5.0 \mathrm{MV} / \mathrm{m}$. The average $\mathrm{Q}$ is $5.5 \times 10^{9}\left(2.4 \times 10^{9}\right.$ specification). This performance suggests that up to $6 \mathrm{GeV}$ can be expected at accelerator start-up. Cryomodule assembly using paired cavities and other components fabricated by industrial vendors is on schedule and continuing at a ratc of two cryomodules per month. As of August 1992, 14 cryomodules had been assembled and installed in the injector and north linac.

The RF system consists of 342 drive systems, a master oscillator, the 5())-keV injector, and five RF separators. All high power amplifiers are installed and in checkout. In recent superconducting injector operation, RF control requirements were achieved, and a prototype RF separator was heam tested.

Cryogenic systems consist of a 4,800 watt, $2 \mathrm{~K}$ Central Helium Liquitier (CHL), an experimental end station refrigerator (ESR), and $2 \mathrm{~km}$ of transfer lines. CHL has operated at $4 \mathrm{~K}$ for more than $7,8(0)$ hours, and $2 \mathrm{~K}$ start-up is in progress. ESR components are 95 percent in hand, and 96 percent of the linac transfer lines are installed.

Accelerator instrumentation and control consists of a 70,(0)()-channel hardware and software system. The system is operational with 85 percen! of the control computers on-site. Beam diagnostics are in final procurement and undergoing installation. The heam position monitors have a resolution of (0.1 mm-microampere. Beam harps have a resolution of hetter than 40 micrometers, with improvements being implemented to reach 10 micrometers.

Beam transport consists of 2,264 magnets and $4.5 \mathrm{~km}$ of beam line vacuum. The east arc is nearing completion, with all dipoles installed. The DC power system's 1,817 trim (low power) supplies $\left(10^{-4}\right.$ regulation) are complete, and 21 box (high power) supplies (10.5 regulation) are in acceptance testing. Regulation tests demonstrate that the supplies meet specifications.

\section{Injector Operations}

The 45-MeV injector, with production hardware and software including 2 cryomodules, has been extensively tested and commissioned on a 24 -hour schedule, validating the basic superconducting accelcrator design and meeting all beam performance objectives. Radio-frequency phase is controlled to within 0.03 degrees, and amplitude to $1.5 \times 10^{-4}$. Bunch lengths under 0.5 degrees are routinely obtained. Measurements have provided excellent agreement with simulations. The injector superconducting components have heen thermally cycled hetween room temperature and $2 \mathrm{~K}$ several times. 


\section{Commissioning Outlook}

Commissioning of the accelerator will proceed in parallel with installation. This optimizes the resource pool and identifies potential difficulties early. Near-term commissioning milestones include low power testing (maximum of $17 \mathrm{~kW}$ ) of the north linac, followed by low power testing of the east arc. High power tests of the north and south linacs are followed by the heginning of single-pass operation and delivery of $800 \mathrm{MeV}$ beam to Hall $\mathrm{C}$ in March 1994. Multiple-pass commissioning follows with operation up to $4 \mathrm{GeV}$. Finally, three-heam distribution tests delivering beam to the three experimental areas would be complete in December 1994.

\section{Potential for Technology Transfer}

The state of the art of SRF technology is being advanced by SRF technical support efforts and by initial accelerator operations for commissioning at CEBAF, the world's first large-scale application of SRF. Because of CEBAF's demonstrated SRF accelerator performance and technical expertise, industry has shown substantial interest in CEBAF SRF technology transfer. 


\section{CONTINUOUS ELECTRON BEAM ACCELERATOR FACILITY}

12000 Jefferson Avenue

Newport News, Virginia 23606

Title of Project: $\quad$ CEBAF Experimental Equipment and Scientific Program

Person in Charge: J. J. Domingo

Telephone:

(804) 249-7587

Scientific Staff: $\quad$ D. J. Abbott, B. Adesanya, O. K. Baker, B. L. Berman, V. D. Burkert, S. C. Capstick, R. D. Carlini, M. Chew, S. V. Gardner, J. L. Goity, J. Gomez, F. L. Gross, W. G. Heyes, N. Isgur, E. A. Jastrzembski, J. J. Lerose, D. J. Mack, S. Majewski, B. A. Mecking, M. D. Mestayer, Z. E. Meziani, S. K. Nanda, J. J. Napolitano, B. Niczyporuk, D. R. Quarrie, A. Saha, E. S. Smith, L. Tang, P. E. Ulmer, W. A. Watson, R. R. Whitney, S. A. Wood, C. Yan, A. S. Yegneswaran, C. J. Zorn

Understanding of the fundamental structure of matter has undergone a profound transformation in the past decade. It is now known that quarks and gluons-not protons and neutrons-are the basic components of nuclei; and they, together with electrons and photons, are the fundamental components of matter. Along with the discovery of the quarks and gluons has come an understanding of their interactions-the "strong interactions"-so that now nuclear and subnuclear physics have, for the first time, a basis as solid as the electron theory on which atomic and molecular sciences are built. The analogy here is deep: the proton and neutron are helieved to be "quark atoms" (bound states of quarks held together hy gluons) just as ordinary atoms consist of electrons bound by photons to the atomic nucleus; nuclei may he considered analogous to molecules, both being relatively weakly bound compounds of their respective "atoms."

Although it is helieved that the modern theory of the strong interaction-QCD--is exact, it has only heen tested in the very high energy regime where the interaction becomes weak so that the cuuarks hehave as nearly free particles. CEBAF's scientific goal is to investigate the transition region hetween this "asymptotically free" high energy regime and the strongly interacting regime most relevant to the world around us, where the underlying physics is poorly understood. CEBAF's 4-GeV electron heam is, in many respects, an ideal prohe for the study of this transition region because the electromagnetic interaction is well understood, and the wavelength of the electron is about one tenth of the nucleon's size. In deep inclastic scattering, the transition from nucleon to quark degrees of frecdom is cmpirically ohserved lo occur at roughly this energy.

To exploit the CEBAF accelerator, the experimental equipment in the three end stations has heen chosen 10 emphasize complementary aspects of the scientific program.

Hell A: The major experimental equipment in Hall A consists of two identical high resolution superconducting magnetic spectrometers having a large solid angle and a maximum momentum of $4 \mathrm{GeV} / \mathrm{c}$. These spectrometers will he utilized for detailed investigations of the structure of light nuclei through inelastic electron scattering in which the knocked-out nucleon is observed in coincidence with the scattered electron. These measurements will extend the range of investigated 
momentum transfers from the present region, where the conventional nucleon-based picture of nuclear structure is adequate to fit the data, to very high momentum transfers, where deviations are expected. The high resolution is required to isolate the different reaction channels in light nuclei so a clean comparison with theory can be achieved. Iligh absolute accuracy is required to separate the various electromagnetic currents.

In addition to the study of light nuclei, the spectrometers will be used to determine the charge and magnetic form factor of the proton and neutron. Although these are known at low momentum transfer for the proton, the electric form factor of the neutron is almost completely unknown. These form factors will test the basic ingredients of microscopic models.

Two key experiments propose to use the Hall A spectrometers for the precision measurement of parityviolating scattering of polarized electrons from a proton target. These measurements will measure (or limit) the strange-quark content of the nucleon that several recent measurements indicate is large. Although the proton is free of strange quark-antiquark pairs in the naive quark model, there is no known fundamental justification for their absence.

All superconducting magnetic components have been ordered, and the Hall A collaboration is in the final stage of detector prototyping. The first spectrometer in Hall $\mathrm{A}$ is scheduled for completion in the spring of 1995 and the second in the summer of 1995.

Hall $B$ : The major apparatus in Hall $\mathrm{B}$ is a large acceptance particle detector based on a toroidal magnetic field produced by six superconducting magnetic coils. The sectors between the coils are instrumented with drift chambers, Cerenkov counters, scintillation hodoscopes, and calorimeters to identify and determine the momenta of several simultaneously emitted charged particles. Scintillation counters together with the calorimeter elements also allow an energy determination for neutral particles. A large international collaboration is fabricating and assembling the detector.

A leading research program for CEBAF Large Acceptance Spectrometer (CLAS) is the investigation of the quark-gluon structure of the nucleon, especially the detailed study of its excited states. The constituent quark model has been very successful in predicting some aspects of the spectra of thesc states; however, a large number of the predicted states has not been observed. There is, moreover, little experimental data on the transition strengths of these resonances; this aspect of the theory is almost completely untested. The history of physics has shown that it is often possible to get correct spectra with incorrect models; transition strengths, that are sensitive to the internal quark structure of the state, will provide much more stringent tests of the model.

The success of the constituent quark model is in any event a puzzle hecause there is an apparent ahsence of states involving gluon excitation. This situation is roughly analogous to proclaiming quantum electrodynamics the correct theory of atomic spectra without ever having observed light. Model ealculations indicate that most of the predicted "gluonic" states decay into many particle modes that would not have heen observed with previous generations of detectors. The hroad acceptance in hoth momentum and solid angle of the CLAS spectrometer will greatly facilitate the search for such "hybrid" states.

Hall $C$ : Hall $\mathrm{C}$ is the area where special experimental apparatus will he mounted to address specific physics questions. To this end, the initial equipment is limited to a magnetic spectrometer of moderate resolution hut extended upper momentum, and a wide-acceptance, Short Orhit Spectrometer (SOS) for use with low momentum particles such as kaons and pions having a short lifetime. 
An important example of a special experiment foreseen for the hall is a precision measurement of parity violation in the scattering of polarized electrons from a proton target. These measurements, using a special detector system heing designed and constructed by a user collshoration led by the University of Illinois, complement the parity-violation experiments in Hall A.

Another topic proposed for detailed investigation is "Color Transparency": the possibility of a greatly decreased nucleon effective interaction strength at high momentum transfer. This phenomenon is predicted to arise from the unique feature of QCD that three quarks at a point can have their strong charges cancel (as could an e+e-pair in electromagnetism).

Experimental equipment in Hall $\mathrm{C}$ is the furthest along in preparation, with the heginning of physics scheduled for April-June 1994. The High Momentum Spectrometer (HMS) cradle is installed, and shipment to CEBAF of the HMS dipole iron is expected this fall, while the dipole coil is expected in the spring of 1993. SOS has been designed by ANL and all major components have heen procured. Lead glass for the HMS calorimeters is in hand, and two large HMS drift chambers are complete and undergoing testing.

The scientific program at CEBAF has major user commitment with more than 700 users from 199 institutions. The user community has committed 395 man-years toward the huilding of experimental equipment. There is high interest in the evolving experimental program. To date, 65 research proposals have been submitted by 325 scientists requesting 2,365 days of heam. Of these, 34 proposals have been approved, representing 783 days of beam, 251 scientists, and 66 research institutions.

The physics research of CEBAF and of the community it serves now encompasses areas from hoth nuclear and what has traditionally been high energy physics. However, in contrast to modern high energy physics, which is concerned with the ultimate laws of nature, the focus of CEBAF research is dynamical: given the laws, how does the real world work?

Our present inability to discover how QCD leads to the confinement of quarks and thence to the structure of nuclear matter stands as the most glaring gap in our understanding of the world around us. CEBAF's principal scientific mission is to use the electron as a probe of strongly interacting matter to close this gap. 


\author{
Physics and Mathematics Group \\ Idaho Falls, Idaho 83415 \\ Title of Fie ject: Nuclear Science \\ Persons in Charge: R. C. Greenwood, C. W. Reich \\ Telephone: $\quad$ (208) 526-9269 \\ Scientific Staff: $\quad$ R. G. Helmer \\ FY 1992 Funding: $\quad \$ 350,000$
}

IDAHO NATIONALENGINEERING LABORATORY

The study of the decay properties of radioactive nuclei is one of the more valuable sources of informati $:$ on low energy nuclear structure. In addition, such information has important applications in a wide range of scientific disciplines and areas of technology.

This program's objectives are the measurement and evaluation of nuclear structure and decay data important for various activities in nuclear science and technology. It emphasizes (1) the meacurement of decay data of importance for long-term data needs of the fission and fusion energy programs and other areas in basic and applied sciences, and (2) the evaluation of nuclear data for the Nuciear Data Sheets and for the Evaluated Nuclear Structure Data File/B (ENSDF/B). Work in this latter area represents the idaho National Engineering Laboratory (INEL) participation in both the International Nuclear Structure and Decay Data (NSDD) Evaluation Network to provide mass-chain evaluations for nuclear structure research and in the Cross Sections Evaluation Working Group to provide evaluated decay data for the U.S. program in reactor research and technology.

The measurement activities currently involve (1) beta-decay properties, primarily of short-lived fissionproduct nuclides; (2) delayed-neutron energy spectra for individual precursor nuclides; and (3) precise gamma-ray emission probabilities. A principal emphasis of this activity is on the use of the Total Absorption Gamma-Ray Spectrometer, located on the ${ }^{252} \mathrm{Cf}$-based INEL Isotope Separation On Line facility, for measurement of heta-decay feeding (beta-strength) distributions of short-lived fissionproduct nuclides. To date, measurements have been undertaken on selected fission-product isotopes of $\mathrm{Rh}, \mathrm{Sr}, \mathrm{Y}, \mathrm{Cs}, \mathrm{Ba}, \mathrm{La}, \mathrm{Ce}, \mathrm{Pr}$, and Nd.

In the area of nuclear data evaluation, the nuclear masses currently assigned to this program are $A=87$ and $153 \leq \mathrm{A} \leq 163$. The rate of production of mass-chain evaluations from this effort satisfies an objective of the NSDD network, namely, a currency of 5 years or less. Our primary responsibility for the ENSDF/B work is to produce evaluated sets of decay data for -900 nuclides including those of the fission-product nuclides. 


\section{LAWRENCE BERKELEY LABORATORY}

Accelerator and Fusion Research Division

Berkeley, California 94720

Title of Project: Bevalac Operations

Porsons in Charge: B. Feinherg, R. A. Gough

Tolephone: $\quad$ (510) $486-5575$

Scientific Staff: $\quad$ C. Celata, W. Chu, B. Feinberg, J. G. Kalnins, G. Krebs, F. Lothrop,

B. Ludewigt, M. McMahan, T. Renner, J. Staples

FY 1992 Funding: $\quad \$ 17,545,000$

At LBL, the SuperHILAC, an: 8.5-MeV/nucleon heavy-ion linac, and the Bevatron, a weak-focusing synchrotron, form a system called the Bevalac. It is run as a national user facility by the LBL A celerator and Fusion Research Division, in cooperation with the Nuclear Science Division. With its unique ability to supply ion beams of any element at $\mathrm{GeV} /$ nucleon energies, the Bevalac serves nuclear and atomic physics, astrophysics, and biomedicine.

SuperHILAC pulses are injected into the Bevatron in a cycle that provides experimenters with a duty factor as high as 75 percent. The ions are accelerated to energies as high as $4.9 \mathrm{GeV}$ for protons, $2.1 \mathrm{GeV} / \mathrm{n}$ for carhon, or $960 \mathrm{MeV} / \mathrm{n}$ for uranium. A local injector is generally used for the lighter ions. The Bevalac can accelerate either fully stripped ions or partially stripped ions with charge-tomass ratios as low as that of $\mathrm{Au}{ }^{11+}$. High quality radioactive heams produced hy fragmentation of the primary heam are also available.

The Bevalac's major detector facilities include a:

- Heavy-Ion Superconducting Spectrometer, a large volume magnetic spectrometer system, with its Equation-of-State TPC.

- Dilepton Spectrometer, a high efficiency detector for e+c- pairs, which are not influenced hy the strong force and are therefore thought to be a "clean" source of information on nuciear collisions.

- Multipurpose projectile-fragment spectrometer.

- Specialized low energy heam line instrumented as a high resolution primary-heam facility, ats well as a radioactive isotope spectrometer and a facility for subthreshold particle production.

Current plans call for operations to stand down in FY 1993 at the end of DOE Division of Nuclear Physics support. Other funding options, centering on its use as a ground-hased cosmic-ray "factory" in research for manned deep-space missions, continue to he explored. 
Title of Project: $\quad 88$-Inch Cyclotron Operations

Person in Charge: T. J. M. Symons

Telephone: $\quad$ (510) $486-5670$

Scientific Staff: $\quad$ C. M. Lyneis, D. J. Clark, R. M. Larimer, R. J. McDonald, Z. Q. Xie

FY 1992 Funding: $\quad \$ 2,775,000$

The 88-Inch Cyclotron is operated as a national facility in support of U.S. DOE programs in basic nuclear science. Ongoing nuclear science research includes nuclear structure, heavy element research, proton-rich nuclei, and nuclear astrophysics and fundamental symmetries. The cyclotron serves researchers from LBL, the national laboratories, universities, and foreign institutions. A Program Advisory Committee meets 3 times a year to review the requests for research time. In addition to basic research, the cyclotron provides a crucial service to organizations involved in the U.S. space program. The space application testing includes heavy-ion beams for computer chip testing, testing of charged-coupled devices, and calibration of instruments for satellites. The cyclotron is typically used by 140 scientists from approximately 30 institutions each year.

The central component is a sector-focused, variable-energy cyclotron fed by an Electron-Cyclotron Resonance (ECR) high-charge-state ion source, the LBL ECR. This versatile combination produces heavy ion-beams from helium to oxygen with energies up to $32 \mathrm{MeV} /$ nucleon. For heavier ions, the maximum energy per nucleon decreases with increasing mass. Typical ions and maximum energy (MeV/nucleon) are argon (20), krypton (8), and xenon (5). Most metallic ions and all other gaseous ions in between these examples have either been accelerated or can be developed as needed, with energies high enough for nuclear physics studies. Light ions - p,d, ${ }^{3} \mathrm{He}$, and ${ }^{4} \mathrm{He}-$ are produced up to total energies of 55, 65, 135, and $130 \mathrm{MeV}$, respectively. Polarized beams at intensities of up to 0.5 microampere are also available.

The Advanced Electron Cyclotron Resonance (AECR) ion source is being brought into operation to provide new capabilities for the cyclotron. The AECR source, which operates at $14 \mathrm{GHz}$ and utilizes an electron gun to boost performance, was developed at LBL to provide higher charge-state ions and more intense beams. The higher charge states result in higher cyclotron energies for heavy ions. AECR has been used to produce krypton beams up to $12 \mathrm{MeV} /$ nucleon for a nuclear physics experiment; test runs have also accelerated xenon to $8 \mathrm{MeV} /$ nucleon and bismuth to $4 \mathrm{MeV} /$ nucleon. The cyclotron is also being upgraded to optimize its performance for heavy-ion beams needed for the scientific research planned with the Gammasphere Detector. Improvements in the cyclotron vacuum system will increase the intensity for heavy ions above mass 100 , and a beam pulse reduction system will improve beam timing.

There is a large complement of equipment located in the experimental areas served by the cyclotron. In particular, a 21-element array of BGO Compton-suppressed high resolution germanium detectors (HERA) is being used for the study of nuclei at high spin. A new detector array, Gammasphere, with 
greatly improved resolving power, is heing constructed at LBL and will be operated initially at the cyclotron for the international nuclear struclure community. Gammasphere will consis: of 110 Compton-suppressed Ge detectors and will he the premiere nuclear structure detectoi system for the 1990s. There is also an on-line isotope separator, 60-inch scattering chamber, an $₫$ a high level cave for bombarding radioactive targets. In addition, there is a separate heam line on the ECR source itself for studies of atomic physics and surface physics using low velocity highly charged ions. 


\section{LAWRENCE BERKELEY LABORATORY}

Nuclear Science Division

Berkeley, California 94720

Title of Project: High Energy Heavy-Ion Research Program

Person in Charge: T. J. M. Symons

Telephone: $\quad$ (510) $486-5670$

Scientific Staff: $\quad$ F. S. Bieser, M. Bloomer, D. Cebra, W. Christie, S. Costa, E. M. Friedlander, P. M. Jacobs, D. E. Greiner, C. R. Gruhn, J. W. Harris, H. Huang, P. Jones,

S. Margetis, D. Keane, P. J. Lindstrom, H. S. Matis, K. Matsuta,

C. P. McParland, J. Mitchell, R. Morse, W. Muller, C. Naudet, G. Odyniec,

D. Olson, A. M. Poskanzer, P. B. Price, G. Rai, J. O. ?asmussen, H.-G. Ritter,

G. R. Roche, I. Sakrejda, J. Schambach, L. S. Schroeder, T. J.M. Symons,

H. Wieman, K. Wilson

FY 1992 Funding: $\quad \$ 6,859,000$

\section{Overview}

Ti. main focus of the future high energy heavy-ion research program at LBL is RHIC, which will be completed at BNL in 1997. For the nearer term, the various activities of LBL at the CERN laboratory in Geneva, Switzerland, have finished data-taking with ${ }^{32} \mathrm{~S}$ beams and have now consolidated into one experiment, NA49, for the $\mathrm{Pb}$ beams program in 1994. In the short term, the extensive local activities at the Bevalac are finishing up in anticipation of the Bevalac shutdown in early 1993.

The goal of many of the Bevalac experiments has been to compress nuclear matter sufficiently to study its equation of state, or how its density responds to the pressure built up in the collision. The higher energy program at CERN is attempting to compress nuclei sufficiently to produce a transition of the nucleons in the nuclei to a plasma of free quarks and gluons. Quarks are the constituents of nucleons and gluons are the force fields that hold them together. At the still higher energies expected at RHIC, the density of the energy of the produced particles will be so high that a momentary state of the quark-gluon plasma is expected. It is believed that the quark-gluon plasma existed soon after the Big Bang at the creation of the Universe and may exist now in the cores of neutron stars.

\section{RHIC Program}

The Solenoidal Tracker At RHIC (STAR) Collaboration now has 135 scientists from 23 institutions. The spokesperson and 30 of the scientists are from LBL. STAR is an experiment to study particle production and high transverse momentum jet production at midrapidity to identify the phase transition from normal nuclear matter to quark matter. A measurement of the produced particles at midrapidity provides the opportunity to select on events with extreme values of temperature (particle spectrum), flavor (strangeness content), shape (particle momenta), and size (two-particle correlations). The experiment will contain a three-dimensional imaging device called a TPC located inside a superconducting solenoidal magnet for tracking, momentum analysis, and particle identification. Timeof-flight detectors surrounding the main TPC will extend particle identification to higher momenta. A detector made of silicon to track the particles near the interaction region will distinguish primary and 
secondary vertices and improve the tracking and momentum resolution. Segmented electromagnetic calorimeters will be implemented in an azimuthally symmetric geometry outside the magnetic field for jet identification and triggering. External TPCs around the beam line will extend the multiplicity and charge-sign measurements to more forward and backward angles. A vigorous detector research and development program is underway, and a conceptual design report describing the detection system and its expected performance was submitted to RHIC management in mid-1992.

\section{CERN Program}

At the CERN Super Proton Synchrotron, the emphasis of the recently completed program of $200 \mathrm{GeV} /$ nucleon ${ }^{32} \mathrm{~S}$-induced reactions was to explore the possibilities of producing a phase transition from hadronic matter to a quark-gluon plasma in central collisions of héavy ions at these energies. LBL effected a consolidation so that at the end of the ${ }^{32} \mathrm{~S}$ data-taking, it was participating in only one CERN experiment: NA35. Its TPC was the first such detector to operate in a magnetic field free region with pad readout only. LBL built and installed 6,000 additional channels of modern electronics. This is a step on the way to a much larger contribution from LBL of integrated electronics for the main TPC of the NA49 Pb-beams experiment in 1994. NA49 will consist of two dipole magnets in series, a large volume TPC with 90,000 channels for tracking and particle identification of charged particles, the NA35 TPCs on one side for the wide-angle pions, two high resolution intermediate-size TPCs to detect neutral strange particle decays, and a time-of-flight wall to complete the hadron identification scheme. This combination of detectors should allow enough coverage of the particles from the reaction to do event-by-event physics for the first time at a CERN experiment. It should be possible to correlate, on an event-by-event basis, such variables as the source radius from two-pion correlations, entropy from rapidity densities, temperature from slopes, and stopping from baryon distributions.

\section{Bevalac Program}

The central focus of the Bevalac's research program continues to be the production and study of extreme conditions in nuclear matter. Early experiments with the Plastic Ball and Streamer Chamber provided the first definitive evidence for collective flow of nuclear matter at high temperatures (50-100 MeV) and nuclear densities (2-4 times normal) created in the central collision of two heavy nuclei. These experiments allow us to study the thermodynamic and transport properties of nuclear matter, and, from this, the nuclear matter equation of state, a quantity of fundamental importance in nuclear physics and of relevance to the understanding of the extreme conditions existing inside neutron stars.

Distant or grazing collisions between nuclei at the Bevalac provide unique opportunities for creating nuclear fragments containing extreme numbers of neutrons and protons, enabling us to study nuclear matter at normal density out to the proton and neutron drip lines. At low and intermediate energies, the Bevalac can produce extremely hot nuclei. These studies focus on the question: How much energy can a nucleus actually hold before it explodes into its constituent parts?

The expected shutdown of the Bevalac in FY 1993 has stimulated a concentration on the central scientific theme and a consolidation to three programs (described later) studying the properties of nuclear matter and an atomic physics experiment. The emphasis is on using unique experimental equipment and beams. 
The Equation-of-State (EOS) TPC, the next-generation $4 \pi$ detector, has completed its construction phase and begun operation for physics in FY 1992. EOS has pioneered the technique of tracking by pad-readout only in a magnetic field and high density electronics using custom-designed integrated circuits. EOS will expand on existing $4 \pi$ measurements and provide new capabilities for complete event analysis of the central collisions of the heaviest nuclei. In addition, in conjunction with the multiple sampling ionization chamber (MUSIC) and with a time-of-flight wall, the experiment is studying multifragmentation and is able to do a complete experiment in charge space.

Rare events can often provide insight into differing aspects of reaction dynamics. It is generally acknowledged that dileptons, because they interact only weakly, are a useful probe of the hot, compressed phase of the collision process. (By contrast, pions and nucleons, which interact strongly with matter, suffer considerable scattering before being detected.) Because dileptons are expected to be extremely rare, special detection techniques are required to sort them out from the myriad of other particles produced by the collision. The Dilepton Spectrometer (DLS) has measured dilepton production in $\mathrm{p}+\mathrm{Be}$ and in $\mathrm{Ca}+\mathrm{Ca}$ reactions at different energies. Bremsstrahlung models are not able to explain even the $p+$ nucleon interactions. The DLS Collaboration has just finished a systematic study of the elementary p-p and p-n interactions at different energies and is continuing the investigation of heavier systems.

The production of radioactive beams and measurements of their ground-state properties were pioneered at the Bevalac by several groups from Japan in association with LBL scientists. Initial work involved the measurement of the radii of light neutron-rich nuclei, culminating in the observation of the "neutron halo" associated with ${ }^{11} \mathrm{Li}$. Measurements of the magnetic moments of polarized unstable nuclei have recently been completed. Present work in this area continues to concentrate on measurements of the correlations between nuclear fragments from these exotic nuclei and to play a worldwide leadership role in defining new directions for this line of research. 


\section{LAWRENCE BERKELEY LABORATORY}

Nuclear Science Division

Berkeley, California 94720

Title of Project: $\quad$ Low Energy Research Program

Person in Charge: $\quad$ T. J. M. Symons

Telephone:

(510) $486-5670$

Scientific Staff: $\quad$ J. Cerny, Y. D. Chan, N. Colonna, P. Fallon, S. Freedman, B. Fujikawa,

A. Garcia, K. Gregorich, D. C. Hoffman, D. Lee, I. Y. Lee, K. Lesko,

A. Macchevelli, D. Moltz, L. G. Moretto, M. Nitschke, E. Norman,

M. J. Nurmia, D. Oliveira, J. Scarpaci, S. Q. Shang, W. Skulski, F. S. Stephens,

M. A. Stephens, R. Stokstad, A. Turler, P. Wilmarth, G. J. Wozniak, I. Zliman

FY 1992 Funding: $\quad \$ 3,398,000$

\section{Overview}

The Low Energy Research Program is a broad and diversified enterprise carried out at three accelerators-the 88-Inch Cyclotron and the Bevalac at LBL and the K1200 Superconducting Cyclotron at Michigan State University (MSU). Basic research in nuclear physics is done with both heavy- and light-ion heams. These probes are used to study nuclear structure, nuclear chemistry, nuclear astrophysics, weak interactions, and heavy-ion reaction mechanisms. Main themes are the effects of high angular momentum, extremes in isospin and nuclear charge (the heaviest elements), exotic modes of decay, nuclear astrophysics and neutrino physics. In addition, there is a strong, ongoing detector research and development program.

\section{Nuclear Structure Studies}

The focus of studies in high spin nuclear structure continues to he on superdeformed (SD) nuclei utilizing the High Energy Resolution Array (HERA). Normal nuclei are typically spherical or slightly deformed. However, SD nuclei have a very large deformation. The HERA array consists of 21 Compton-suppressed germanium detectors, together with an inner ball of 40 BGO sectors that give the multiplicity and the sum energy of the gamma-rays emitted from excited nuclei. During the last several years, many new SD hands have been discovered at the cyclotron using the HERA array. A major effort has been undertaken to map out the houndaries of superdeformation in the region of nuclei around $A \sim 19()$, where about 25 SD bands have been experimentally observed. With the discovery of an SD hand in ${ }^{198} \mathrm{Ph}$, this region of superdeformation has heen extended to $N=116$. Many of these hands show similar moments of inertia and "identical" transition energies. With the discovery that a nucleus may contain more than one superdeformed hand, an intensive effort has heen undertaken to find examples of such multiple SD hands.

Gammasphere will be the premiere nuclear structure detector system for the 199()s. It is a major equipment project that is heing huilt at LBL and will he initially operated at the 88 -Inch Cyclotron for the international nuclear structure community. Gammasphere will consist of 110 Compton-suppressed Ge detectors and can he used with auxiliary particle detectors. This more powerful array will greatly 
facilitate the study of weakly populated SD hands and allow the investigation of superdeformation in new regions previously inaccessible with HERA and other existing arrays.

\section{Heavy Elements}

The discovery and study of transuranic elements has a long and illustrious history at LBL. Several factors have combined to make the 88 -Inch Cyclotron facility one of the leading laboratories in the world for the production and study of transuranic nuclei. One factor is the availability of intense heams of neutron-rich projectiles. Another is the technology developed at LBL and LLNL for producing, handling, and irradiating radioactive targets of isotopes from uranium through einsteinium. Currently, three aspects-the optimal reaction mechanism for producing transuranic elements, the chemistry of the short-lived atoms, and the properties of their nuclear decay-are under investigation. Recently, the Heavy Element group and its collaborators have been investigating the chemical properties of the heaviest elements. The chemistry of atoms with large atomic numbers is influenced by the effects of relativity on the valence electrons that could affect their chemical properties. Several experiments on the chemistry of ${ }^{261} \mathrm{Rf}$ (element number 104) have been conducted. Results show that $\mathrm{Rf}$ behaves similarly to the group IV element $\mathrm{Zr}$. In addition, a new nonaccelerator program to study the chemical behavior of plutonium in groundwater has also been undertaken.

In February of 1991, the Glenn T. Seaborg Institute for Transactinium Science was jointly established by LBL and LLNL. The Institute will be devoted to the study of the transactinium elements. Special emphasis will be placed on the education and training of future generations of scientists in heavy element research. Professor Darleane Hoffman has been appointed as the first Director.

\section{Reaction Mechanism Studies}

The utilization of heavy-ion reactions to produce hot nuclei and possible exotic nuclear structures is a subject of broad interest at the cyclotron and other accel srators. One approach is to use heavy heams on a light target-reverse kinematics-to increase the sensitivity to rare decay processes. One such process is the emission of complex fragments that are intermediate in mass to light charged particles and fission fragments. Recently, beams of ${ }^{63} \mathrm{Cu}$ have been used with a ${ }^{12} \mathrm{C}$ target to measure, for the first time, a complete set of mass-asymmetric harriers for ${ }^{75} \mathrm{Br}$. The extracted harriers are in good agreement with predictions of the Finite-Range Model. More recently, these measurements have heen extended to heavier systems using neutron-poor $\left({ }^{78} \mathrm{Kr}\right)$ and neutron-rich $\left({ }^{86} \mathrm{Kr}\right)$ heams from the Advanced ECR source. Utilizing a ${ }^{12} \mathrm{C}$ target, two very similar compound nuclei were made, one on the neutron-poor $\left({ }^{90} \mathrm{Mo}\right)$ side and the other on the neutron-rich $\left({ }^{98} \mathrm{Mo}\right)$ side of the valley of stability. At the same excitation energy and angular momentum, the yield of complex fragments from the neutron-poor nucleus is a factor of 10 higher than from the neutron-rich one. Studies of multifragment emission are carried out with higher energy, heavier heams at the MSU K1200 Cyclotron and the LBL Bevalac in the energy regime of 30-100 MeV/nucleon.

In other reaction studies, fragmentation of the target has been studied using radiochemical techniques and a wide range of hombarding energies at the cyclotron and at other accelerators. Also, much lighter projectiles, such as ${ }^{16} \mathrm{O}$ and ${ }^{20} \mathrm{Ne}$ at $32 \mathrm{MeV} /$ nucleon, have heen used to produce projectile-like nuclei with excitation energies up to about $5 \mathrm{MeV} /$ nucleon and observe their decay into as many as five fragments. 


\section{Exotic Nuclei Far From Stability}

The spectroscopy of nuclei far from stability begins with the production and identification of a new nuclide, proceeds to the measurement of its mass and decay properties, and ultimately to the study of its (or its daughter's) level structure. The new isotopes ${ }^{252} \mathrm{Bk}$ and ${ }^{253} \mathrm{Bk}$ have been recently observed at the 88-Inch Cyclotron. The OASIS group has studied neutron-deficient nuclei in the region $\mathrm{Z}=54-71$. Using their on-line isotope separator, this group as investigated the decay properties of ${ }^{155} \mathrm{Lu},{ }^{155} \mathrm{Yb}$, ${ }^{155} \mathrm{Tm},{ }^{151} \mathrm{Er}$, and ${ }^{151} \mathrm{Ho}$. In a study of the alpha emitters above $\mathrm{N}=82$, isomer excitation energies and precise alpha-branching ratios were measured. Lighter and shorter-lived proton-rich nuclei are also studied at the 88-Inch Cyclotron. The heaviest member of the $T_{z}=-3 / 2, A=4 n+1$ series, ${ }^{65} \mathrm{Se}$, has been produced and its beta-delayed proton emission observed.

During development of the 1989 Long Range Plan for Nuclear Science, LBL suggested the construction of a high intensity Radioactive Nuclear Beam (RNB) facility in the U.S. This idea found broad national and international support. In October of 1989, LBL organized the First International Conference on Radioactive Nuclear Beams. This was followed by a Workshop at Los Alamos and the second international RNB conference in Louvain, Belgium, in 1991. A Steering Committee was formed with representatives from major North American laboratories, including LBL. Recently, the Steering Committee issued a white paper in which the scientific case for an RNB facility in North America is made and design goals are put forth. The proposed facility has tentatively been given the name Isospin Laboratory (ISL). Currently, the following ISL-related questions are under investigation at LBL: the optimum method for producing high intensity, high energy, light-ion primary beams, expected activity and radiation levels and shielding requirements, ion sources for on-line operation, and the optimum method for accelerating low velocity, low charge-to-mass-ratio ions. In addition, there is ongoing effort to calculate RNB yields for ISL and to perform computer evaluations of high intensity targets.

\section{Neutrino Physics}

In collaboration with 11 other institutions from Canada, the U.S., and the United Kingdom, LBL is participating in the Sudbury Neutrino Observatory (SNO). SNO is a heavy water neutrino detector designed to address the solar neutrino problem, the question of neutrino oscillations, and neutrino production in stellar collapse. SNO will be located more than 1 mile underground in a mine in Sudhury, Ontario. The U.S. is providing a portion of the funding for this project. LBL is designing the structure to support the approximately 10,000 phototubes that will record Cerenkov light from neutrino-induced reactions. In addition, the LBL group has assumed major responsibility for limiting contamination due to low-level background radioactivity.

Another LBL group has automated a telescope at the Leuschner Observatory for supernova detection. During the past several years, many supernovae have been discovered with this automated telescope. Recently, a measurement of supernova rates has been completed. The surprising result is that the rate of supernovae is substantially greater in galaxies similar to our own than was expected. The higher rate is a direct consequence of the greater sensitivity of the present technique compared to those used in precious searches. One of the conclusions of this work is that our galaxy should have a supernova, on average, once every 30 years. This result has encouraging consequences for future neutrino and gravitational-wave observatories.

During the last year, a new group was formed to study weak interactions. The discovery that certain neutral atoms can be cooled and manipulated with laser light provides the exciting opportunity to 
study nuclear beta decay with unprecedented precision. In traps, atoms are essentially free, so a trap is like an atomic beam, but without the unpleasant limitations arising from motion along the beam axis. Moreover, trapped atoms can easily be polarized without the limitation of wall relaxation inevitable when atoms are stored in cells. The beta-decay asymmetry of ${ }^{21} \mathrm{Na}$ will be measured for the purpose of studying the structure of the charge-changing weak currents and to test the hypothesis that the weak interaction should be represented by a purely left-handed theory at the most fundamental level. Previously, the trapping of cold ${ }^{23} \mathrm{Na}$ has been demonstrated. An atomic beam of ${ }^{21} \mathrm{Na}$ will be extracted from an oven, cooled with laser light, and then trapped using the techniques developed with stable sodium. 


\section{LAWRENCE BERKELEY LABORATORY}

Nuclear Science Division

Berkeley, California 94720

Title of Project: Isotopes Project

Person in Charge: T. J. M. Symons

Telephone: $\quad$ (510) 486-5670

Scientific Staff: $\quad$ E. Browne, R. B. Firestone, V. S. Shirley, B. Singh, C. M. Baglin

FY 1992 Funding: $\quad \$ 663,000$

The lsotopes Project compiles, evaluates, and disseminates nuclear structure and radioactive-decay data for hasic and applied research and for diverse technical applications. Since 1979, the project has coordinated its efforts with the national and the international nuclear data networks and is responsible for (1) evaluating properties of nuclei with mass numbers $A=89$ to 93, 167 to 194, 206, 210, 211, 212 , $215,219,223$, and 227 and (2) converting data with $A=33$ to 44 into a standard network format. This responsibility includes preparing data in computerized form for entry into the Evaluated Nuclear Structure Data File (ENSDF). Comprehensive evaluations, produced from ENSDF, are subsequently published in the journal Nuclear Data Sheets.

Spectroscopic data from radioactive decay and nuclear reactions-after verification of completeness, correctness, and self-consistency - serve as input data for determining recommended adopted values of specific nuclear properties. These "best values," deduced with the aid of statistical procedures, the application of systematics, and the use of nuclear models, constitute the main scientific contribution of the data evaluation effort. The project's data and corresponding bibliographic references are both computer retrievable and available in published form.

Concurrent with evaluation of data, the Isotopes Project develops methods and computer codes for data analysis. These include minimization procedures for deducing hest values from various sets of data and data verification codes for assuring correctness and uniformity. The project has a continuing interest in methods for propagation of the experimental uncertainties reported for nuclear properties. Their application in nuclear data evaluation leads to uniform and rigorous interpretation of the data and results in consistently reliable recommended values.

The Isotopes Project produced seven editions of the Table of Isotopes from 1940 to 1978, the sixth and seventh in book form, and currently is preparing an eighth edition. This new edition will focus on nuclear structure data from reaction and radioactive decay studies and will be available in print and on various computer media. Each edition of the Table of Isotopes provided a comprehensive and critical evaluation of the known nuclear properties deduced from radioactive decay and reaction data. The project is also responsible for the production of the Table of Radioactive Isotopes, first published in 1986. This hook provides a concise source of recommended data derived from ENSDF and is tailored to the needs of applied users in industry, biology, medicine, and other fields. It has also proved itself as an indispensable reference for nuclear physicists and chemists in basic rescarch.

The Isotopes Project has started the development of electronic access to ENSDF on various computer media, which will include personal computers and workstations in the near future. The program 
operates from a chart-of-the-nuclides graphical interface to perform full numerical data searching. Preliminary versions are expected to he released in 1992.

The Isotopes Project serves a broad user community and plays an active role in promoting the science of nuclear data evaluation. It has developed, and makes available, an extensive computerized datahase of nuclear structure and radioactive decay information (LBL/ENSDF) based on ENSDF. 


\section{LAWRENCE BERKELEY LABORATORY}

Nuclear Science Division

Berkeley, California 94720

Title of Project: Nuclear Theory Program

Person in Charge: M. Gyulassy

Telephone: $\quad$ (510) $486-5239$

Scientific Staff: $\quad$ G. Batko, S. Chapman, D. Dong, K. Eskola, N. K. Glendenning, T. Guhr, C. Jarzynski, W. D. Myers, J. Randrup, W. J. Swiatecki, X. N. Wang

FY 1992 Funding: $\quad \$ 1,085,000$

The program includes a broad range of topics on microsccpic and macroscopic nuclear models, nuclear astrophysics, nuclear transport theories, the theory of nuclear phase transitions, the equations of state of dense nuclear matter, QCD and hadrodynamic models of nuclear phenomena, and the order-to-chaos transition in nuclei.

One of the major thrusts of the program is to develop precise transport theories to address a wide range of problems in the area of nuclear collisions over a wide energy range ( $1 \mathrm{MeV} /$ nucleon to $1 \mathrm{TeV} /$ nucleon). An important recent focus has been the development of a detailed Monte Carlo model (HIJING) including low $\mathrm{P}_{\mathrm{T}}$, minijet and hard QCD processes for applications to RHIC. The model has been tested successfully on a wide body of data on pp, pA, and AA data and makes specific new predictions for the RHIC domain, especially on the role of gluon shadowing and jet quenching. In the AGS and Super Protun Synchrotron (SPS) domain, work has concentrated on the problem of nuclear stopping and associated signatures. A wide variety of signatures of quark-gluon plasma functions including pion interferometry, $\mathrm{J} / \Psi$ suppression, direct photon production, antibaryon suppression, and transverse flow continue to be under active investigation. This work is also complemented by field theoretic studies of the nuclear equation of state, models of confinement and chiral symmetry, and QCD perturbative dynamics in nuclei.

Another major thrust is nuclear astrophysics concentrating on the properties of rotating neutron stars. New limits on absolute rotation rate for conventional neutron stars have been calculated. Proposals for strange quark stars that could exceed that limit have been advanced. A new theory of complex phase transitions has been developed for application to neutron star cores. Phenomena such as glitches continue under active investigation.

Another ongoing project is the development of statistical models and dissipative dynamics for understanding multifragmentation phenomena. A general theory of correlated fluctuations and the spinoidal decompositions is under development for applications to nuclear disassembly. The role of multibody forces in dynamics in the Bevalac energy range is also being investigated.

Recent work on macroscopic models of nuclei has focused on the curvature correction to nuclear surface energies and development of a more refined Thomas Fermi model for nuclei. Work on the theory of chaos has resulted in the discovery of a new universal asymptotic velocity distribution for irregular time-dependent potentials. 
Work on high spin nuclear states, neutron-rich nuclei, high energy electron nuclcus interactions, quantum chaos in many-body systems, and neurocomputation techniques for pattern recognition illustrates some of the other directions of current research. 
Department of Physics

Livermore, Califomia 94550

Title of Project: Heavy-Ion Reactions

Person in Charge: J. H. Thomas

Telephone: (510) $422-3434$

Scientific Staff: $\quad$ H. C. Britt, J. B. Costales, L. F. Hansen, T. C. Sangster, S. Tonse

FY 1992 Funding: $\quad \$ 150,000$

This is a growing program that focuses on experimental studies of heavy-ion reactions at relativistic energies. The emphasis is on the use of heavy-ion collisions to study the properties of nuclear systems at temperatures and densities far removed from those available in low energy nuclear reactions.

We are members of the E802 Experiment at AGS and the PHENIX Collaboration at RHIC.' The goals of the E802 collaboration include a systematic study of large nuclear systems naade of hot, high density hadrons. Our primary role has been to expand the inclusive studies begun in the initial experiments by measuring protons, deuterons, and tritons at angles from 50 to 130 degrees. These data will be used to further constrain the theoretical models that describe target-spectator excitations.

The goals of the PHENIX Collaboration are to observe the quark-gluon plasma. We are working on the design of this detector with special emphasis on the magnet and the physics that can be addressed with electrons and hadronic probes of the plasma. The detector is scheduled to go on-line in 1997. 


\section{LAWRENCE LIVERMORE NATIONAL LABORATORY}

Physical Science Division

Livermore, California 94550

Title of Project: Positron-Electron Spectroscopy in Heavy-Ion Collisions

Person in Charge: T. Cowan

Telephone: $\quad$ (510) 423-3935

Scientific Staff: $\quad$ M. W. Clark, V. Horvat, R. R. Rohatgi, R. L. Watson, D. H. G. Schneider

FY 1992 Funding: $\$ 80,000$ :

A major puzzle in nuclear physics is the appearance of the narrow peaks in the energy distributions of positrons and electrons emitted in very heavy nuclear (e.g., uranium + uranium) collisions. Using a new high efficiency spectrometer, we have begun a systematic investigation of correlated positron-electron emission in a wide range of collision systems at the Texas A\&M K500 Cyclotron. In our first experiment, we discovered that the narrow peaks also occur in much lighter (xenon + gold) collisions, indicating that the strong collisional electric field may not be as important to the peak production mechanism as previously believed. Upcoming experiments will explore the role of nuclear reactions in the peak production process and search for positron-electron peaks in even lighter collisions. 
Physics Department

Livermore, California 94550

Title of Project: Bound-State Beta Decay

Person in Charge: $\quad$ D. A. Knapp

Telephone: $\quad$ (415) 422-1023

Scientific Staff: $\quad$ R. Marrs, S. Elliott

FY 1992 Funding: $\quad \$ 110,000$

When a nucleus undergoes beta decay, it emits an electron and an electron antineutrino. In most beta decays, both particles leave the atom. However, there is a small probability that the electron can be created in a bound state of the daughter atom, in which case only the neutrino is emitted. This process is known as bound-state beta decay.

In most cases, the effect of bound-state beta decay on the total beta decay rate is negligible. There are, however, a few nuclei in which bound-state beta decay is the only possible decay process. These isotopes, normally stable when neutral atoms, undergo bound-state beta decay when all of their atomic electrons are removed. The energy required to make the beta decay energetically allowed is provided by the binding energy of the atomic state in which the electron is created. For example, neutral ${ }^{163} \mathrm{Dy}$ is completely stable, but is predicted to decay to ${ }^{103} \mathrm{Ho}$ with a half-life of about 52 days when completely ionized. Another potentially important example is the bound-state beta decay of ${ }^{205} \mathrm{~T} 7$ to ${ }^{205} \mathrm{~Pb}$, a process that could provide information crucial to a geochemical solar neutrino measurement using these isotopes.

We have built a device, known as the Super Electron Beam Ion Trap (Super-EBIT), that can completely ionize Dy atoms and trap them for several hours. During this time, the ions continually emit photons from radiative electron capture. These photons are monitored to observe the buildup of Ho atoms from bound-state heta decay.

The Super-EBIT trap was first run in May 1992. With it, we have produced and trapped bare Dy ${ }^{63+}$ ions with a 150-keV electron beam. Modifications are now underway to improve the ionization balance and trapping time. We anticipate that we will soon observe the process of bound-state heta decay. 


\section{LAWRENCE LIVERMORE NATIONAL LABORATORY}

Computational Physics Division

Livermore, California 94550

Title of Project: Development of Advanced Models for Nuclear Data Evaluation

Person in Charge: $\quad$ R. M. White

Telephone: $\quad$ (510) $422-9668$

Scientific Staff: $\quad$ H. M. Blann, D. A. Resler

FY 1992 Funding: $\$ 245,000$

Nuclear cross sections determine the probabilities for nuclear reactions. They are crucial in the application of nuclear physics to the development of fission and fusion reactors, to astrophysics research in determining the synthesis of elements in stars, and in the application of nuclear technology in areas ranging from national security to radiation oncology. These applications depend upon extensions to current evaluated nuclear data libraries beyond that which has been experimentally measured. To apply nuclear physics to new technologies, we must be able to provide new data evaluations. Because nuclear cross section data are, for the most part, no longer being measured, we are improving current computer models and developing new ones to provide the needed cross section information.

In our modeling of nuclear reaction cross sections for the light nuclei $(A<20)$, we couple together nuclear structure (shell model) calculations with a generalized reaction (R-matrix) formulation. Current work is directed toward improving our understanding of the effective nucleon-nucleon interaction used as the starting point for these cross section calculations. This method can be used to obtain practical results for fusion and astrophysics applications and can further our fundamental understanding of the nuclear force. For $A>20$, we are concentrating our effort on compound and precompound nuclear models and evaluated files of nuclear input parameters. Intercomparison of nuclear modeling results by statistical methods has shown that the ability to calculate cross sections accurately is presently limited more by the uncertainty in input parameters than by the formalism. Therefore, we are constructing new evaluated parameter files for the major constituents of input to reaction modeling.

A new area of interest involves nuclear data needed for medical applications. We have identified the most important reaction cross sections needed up to $70 \mathrm{MeV}$. Part of our future effort will be directed toward providing complete evaluated files to these energies for use in neutron therapy. 


\section{LAWRENCE LIVERMORE NATIONAL LABORATORY}

Physics Department

Livermore, California 94550

Title of Projects: $\quad$ (I) Nucleon-Nucleon Bremsstrahlung, (II) Nuclear Astrophysics as a Probe of Matter at High Temperature and/or Density

Persons in Charge: (I) V. R. Brown, (II) G. J. Mathews

Telephone:

(510) 422-4092

Scientific Staff: $\quad$ C. R. Alcock, P. L. Anthony, S. D. Bloom, M. B. Aufderheide, N. Gentile, H. Kurki-Suonio, J. L. Hughes, M. T. Ressell, N. J. Snyderman

FY 1992 Funoiing: $\quad$ (I) $\$ 71,000$, (II) $\$ 54,000$

The two main goals of Project I are to map the nuclear interaction into regions unexplored by nucleonnucleon elastic scattering and to study the radiation from internal mesons. Because the electromagnetic interaction is well known, the radiation of a photon in nucleon-nucleon scattering (nucleon-nucleon bremsstrahlung) provides a calculable tool for exploring the new region. This new information is required in any process involving more than two nucleons, such as in a nucleus. We obtain good agreement with the data that have the greatest sensitivity to the unexplored region, the proton-proton bremsstrahlurig data from Tri-University Meson Facility (TRIUMF). The second project goal involves neutron-proton bremsstrahlung, which is sensitive to photon emission from internal mesons which mediate the nuclear force. Such radiation effects are very important in electronuclear physics at Bates and CEBAF. Our work, which shows large contributions from the internal mesons, has been crucial in understanding recent unexpected results in yet another discipline, heavy-ion experiments. In the next year, we will be directly verifying this effect in the neutron-proton bremsstrahlung measurements being made at the "white neutron source" at LAMPF.

The goal of Project II is to constrain properties of nuclear matter at extreme conditions of temperature and density from observable effects in astrophysical environments. One environment is that of the first few moments of the Big Bang, where phase changes in matter may influence the production of light and heavy elements by nuclear reactions. Another astrophysical environment is in stellar supernova explosions. In the core of the star, nuclear phase changes may affect the observed energy and neutrino emission from the collapse. Just outside the core, properties of nuclei far from stability will similarly affect the energy and neutrinos from the explosion as well as abundances of heavy elements produced. Our theoretical investigations include (1) the evolution of phase transitions in the early universe and stellar cores, (2) the formation of elements in the Big Bang and supernovae, and (3) shell model computations of nuclear properties for unstable nuclei. New results include constraints on the QCD phase transition, the high density nuclear equation of state, nuclear masses, deformations, and electron-capture rates away from stability. In the next year, we will constrain properties of the electroweak phase transition, develop a more realistic equation of state, and calculate weak nuclear scattering. 
Medium Energy Physics Division

Los Alamos, New Mexico 87545

\author{
Title of Project: $\quad$ LAMPF Accelerator Facility \\ Person in Charge: P. Barnes \\ Telephone: $\quad$ (505) 667-2000 \\ Scientific Staff: J. Bradbury, A. Browman, J. Donahue, W. Harris, D. Fitzgerald, M. Hoehn, \\ E. Hoffman, K. Jones, J. Knudsen, R. Macek, M. Plum, L. Rybarcyk, R. Ryder, \\ O. van Dyck, R. Werbeck
}

FY 1992 Funding: $\quad \$ 41,600,000$

The heart of LAMPF is an $800-\mathrm{MeV}$ proton linac that simultaneously produces three beams: $\mathrm{H}^{+}, \mathrm{H}^{\text {; }}$, and polarized $\mathrm{H}^{\text {. The }} \mathrm{H}^{+}$beam (1-mA average current, $800-\mathrm{kW}$ heam puwer) is used to produce secondary beams of pions, muons, neutrinos, and spallation products used in basic nuclear and particle physics research. The beam can also be used for applied research and development purposes such as the production of significant quantities of medical radioisotopes and the study of radiation effects on materials.

The polarized $\mathrm{H}^{-}$heam is used for nucleon-nucleon and nucleon-nucleus studies and production of polarized neutron beams. This polarized beam is available at energies between 211 and $800 \mathrm{MeV}$ with a current of 0.1 to $1.0 \mathrm{~mA}$. The unpolarized $\mathrm{H}^{-}$beam is used (1) for atomic structure studies of the $\mathrm{H}^{-}$ion, (2) to fill the Proton Storage Ring for delivery of about $80 \mathrm{~mA}$ to the Los Alamos Neutron Scattering Center (LANSCE), and (3) to deliver $1 \mathrm{~mA}$ to the Weapons Neutron Research (WNR) facility. At LANSCE, a very-low-duty, high intensity proton heam impinges on a target to produce short, intense bursts of neutrons used for condensed matter physics, materials science, and biological structures studies. Both LANSCE and WNR are funded from other sources.

During a typical year, LAMPF provides beams for about 5 months. In the LAMPF research program, about 300 scientists and students from more than 50 institutions participate in about 40 to 50 experiments that receive heam. About 10 Ph.D. degrees based on LAMPF research are granted each year.

The following four areas of nuclear physics research receive major emphasis at LAMPF: (1) the interaction of pions with target nuclei to investigate nuclear structure and the nature of the pion interaction in nuclei; (2) proton and neutron reactions with nuclei to investigate spin and isospin degrees of freedom; (3) the basic proton-proton, neu(ron-neutron, and pion-nucleon interaction; and (4) fundamental aspects of the weak interaction. 
Medium Energy Physics Division

Los Alamos, New Mexico 87545

Title of Project: Pion-Reaction Physics at LAMPF

Person in Charge: $\quad$ R. L. Boudrie

Tolephone: $\quad$ (505) 667-6991

Scientific Staff: J. F. Amann, J. D. Bowman, P. A. M. Gram, S. J. Greene, M. B. Johnson, M. J. Leitch, J. B. McClelland, C. L. Morris, J-C. Peng

FY 1992 Funding: $\quad \$ 1,100,000$

This research program aims to describe the scattering and interaction of pions with nucleons and nuclei. LAMPF energies span the range in which the pion combines with nucleons to form the delta resonance with a large probability. Thus, much of pion-reaction physics is devoted to learning how the delta resonance propagates and interacts in nuclei. This effort utilizes a broad array of powerful experimental tools such as the Energetic Pion Channel and Spectrometer (EPICS), the short-flight-path Clamshell spectrometer, a superconducting RF cavity, the Large Acceptance Spectrometer, the BGO Ball, the Neutral Meson Spectrometer (NMS), and an array of polarized targets.

Current research is focused on measuring and interpreting a variety of reactions chosen to exhibit some particular feature of the reaction mechanism. These include pion reactions that excite the nucleus without changing its charge, those in which the pion changes its charge by one unit, double-chargeexchange reactions that are especially sensitive to how nucleons behave when they come close together in nuclei, and reactions where the pion is absorbed within the nucleus. Three beam lines are used to address these studies over a broad range of pion energies.

Reliable systematic data have now been taken on the double-charge-exchange reaction over the full range of pion energies available at LAMPF. Cross sections have been measured to less than a nanobarn per steradian in search of bound states below the pion production threshold. Pion absorption data using the BGO Ball have been taken from 30 to $500 \mathrm{MeV}$ with good energy resolution, demonstrating the utility of multiparticle final-state detection systems at LAMPF. High resolution studies in the resonance region allow the special properties of the pion, such as the strong isospin dependence of its interaction with nucleons, to be exploitt 7 is a sensitive probe of nuclear structure. EPICS provides this capability and is unique in the world. A newly implemented superconducting radiofrequency cavity (SCRUNCHER) has been used to compress the momentum spread of the low energy pion beam, thereby providing high resolution, high count rate capability for pion-reaction studies at low energy. A NMS is being assembled and commissioned to detect neutral pions and etas with high efficiency, large solid angle, and good resolution. On the theoretical side, effort is being concentrated on models that unify inelastic and charge-exchange reactions, so that information obtained in reaction studies can he utilized to advantage in nuclear structure investigations. 
Medium Energy Physics Division

Los Alamos, New Mexico 87545

Title of Project: Nucleon-Nucleon Interaction Studies at LAMPF

Person in Charge: M. W. McNaughton

Telephone: (505) 667-3442

Scientific Staff: $\quad$ K. Johnston

FY 1992 Funding: $\quad \$ 400,000$

The nucleon-nucleon (NN) interaction is of fundamental importance, both as a test of stronginteraction theory and as an essential prerequisite for understanding the nucleus. One of the most basic questions, for example, is whether a nucleus can be described completely in terms of the interactions between its constituent nucleons, or whether the NN interaction is modified within the nuclear medium. There is no chance of addressing this question unless the behavior of free nucleons is well understood. Such a comprehension is essential for the interpretation of experiments that will be performed at CEBAF.

The goals of the NN program at LAMPF have been defined at several recent workshops as follows: to determine the $\mathrm{NN}$ amplitudes at energies up to $800 \mathrm{MeV}$, in order (1) to understand the coupling between $\mathrm{NN}$ and $\pi \mathrm{N}$ interactions near the major pion production thresholds, (2) to provide accurate (1 percent) calibration standards for nuclear and particle physics experiments, and (3) to provide the fundamental NN data needed for nuclear calculations. The data are parameterized in terms of a "phase-shift analysis" to give reliable interpolations as a function of angle and energy. Computer codes are maintained throughout the world to give physicists "instant" predictions for any NN observable or amplitude.

Almost all amplitudes at many energies up to $800 \mathrm{MeV}$ have now been reliably determined to 5 percent by a collaboration of some eight institutions. There remain, however, two anomalies or loose ends: (1) Some aspects of the energy-dependent structure near the delta threshold are not predicted by current theories; measurements near $730 \mathrm{MeV}$ should clarify this. (2) Previous measurements of the $\mathrm{np}$ analyzing power appear to be incorrect; this hasic polarization parameter is widely used as a calibration standard, and so this discrepancy must be decisively resolved. 
Medium Energy Physics Division

Los Alamos, New Mexico 87545

Title of Project: $\quad$ Neutrino Physics al LAMPF

Person in Charge: $\quad$ D. H. Whitc

Telephone: $\quad(505) 667-6375$

Scientific Staff: $\quad$ R. Bolton, R. Burman, J. Donahuc, F. J. Federspiel, G. Garvey, W. Louis, J. Margulies, V. Sandherg, M. Schillaci, R. Werheck, D. Whitehouse

FY 1992 Funding: $\quad \$ 9(0),(0)()$

E1173: The Liquid Scintillator Neutrino Detector, involving a collaboration of 14 institutions, is presently under construction and is expected to take data in FY 1993 after hecoming operational in April of that year. The detector consists of a tank of dilute liquid scintillator of hutyl PPD in mineral oil in which hoth Cerenkov light and scintillator light will he observed hy an array of $1,2(0)$ phototubes. The first experiment with this detector has the goal of searching concurrently for neutrino oscillations in the two processes: (1) a muon neutrino decaying in flight to an electron neutrino, and (2) an electron antincutrino decaying at rest to a muon antineutrino. It is planned also to upgrade the A6 heam stop to increase the decay-in-flight neutrino flux hy a factor of 5 , which will make possible a precision measurement of nmp elastic scattering. This cross section at low energy depends almost completely on the axial vector coupling to the proton $\mathrm{G}_{\mathrm{A}}$, which in turn depends on the contribution of strange quarks to the spin of the proton. This measurement of the contribution of strange quarks is unique and can only he done at low energy at LAMPF.

E1213: Measurements have heen made of the solar neutrino flux hy an experiment using perchlorethylene in the Homestake mine and found to he less than that predicted by the accepted solar model. This LAMPF experiment is intended to measure a similar neutrino reaction on iodine to provide a cross section measurement for an extension of the chlorine experiment. The chlorine cross section will also be measured to provide verification of that value derived from theoretical considerations. 


\section{LOS ALAMOS NATIONAL LABORATORY}

Medium Energy Physics Division

Los Alamos, New Mexico 87545

Title of Project: $\quad$ Muon Decays at LAMPF

Person in Charge: $\quad$ D. H. White

Telephone: $\quad(505) 667-6375$

Scientific Staff: $\quad$ M. Cooper, G. Hogan, R. Mischke, D. Whitehouse, M. Kroupa

FY 1992 Funding: $\quad \$ 8(0), 00()$

E969: The process $\mu \rightarrow \mathrm{e}+\gamma$ has never heen observed; this nonappearance is explained by an ad hoc invocation of lepton family number conservation. An experiment known as MEGA is under construction to search for this process down to a branching ratio near $10^{-13}$. The experiment involves faculty and students from 12 institutions, and new upper limits to the branching ratio are expected to be obtained in 1992 and 1993.

This detector is designed to detect both a positron and a gamma-ray in different parts of the apparatus and measure their energies and directions with high precision. Evidence for this decay mode would require observation of both a positron and a gamma-ray in a restricted energy range in opposite directions in time coincidence. The resolution of the apparatus is crucial to achieving high rejection of other processes that might otherwise be misinterpreted. The apparatus is housed in a solenoidal B field generated by a large aperture superconducting magnet. This B field is sufficiently intense that positrons spiral tightly and do not traverse the photon detector. The photon detector detects photons through pair production and then measures the momentum of the two particles of the pair by reconstructing the trajectories. This separation of function allows the necessary precision to be achieved under conditions of very high muon count rates in the detector.

E1240: The MEGA apparatus described previously will also be used to measure the shape of the muon spectrum. This spectrum is characterized by a parameter rho, which has a value of 0.75 in the standard electroweak theory of muon decay. The mechanical precision of the positron part of the MEGA detector is well suitec to, an accurate measurement of the rho decay parameter. Variation of the spectrum shape from that predicted by the standard theory will indicate physics beyond that usually assumed, namely three lepton families and the $S U(2)_{L} X U(1)$ set of vector bosons. 


\section{LOS ALAMOS NATIONAL LABORATORY}

Medium Energy Physics Division

Los Alamos, New Mexico 87545

Title of Project: $\quad$ Tests of Fundamental Symmetries in Nuclei

Person in Charge: $\quad$ D. H. White

Telephone: $\quad$ (505) 667-6375

Scientific Staff: $\quad$ J. D. Bowman, Y. Fen, J. Knudsen, S. Penttila, S. H. Yoo

FY 1992 Funding: $\quad \$ 400,000$

E1183: $\mathrm{H}^{-}$beam from LAMPF is injected into the Proton Storage Ring and used to provide a neutron beam that is energy selected by time of flight. This beam of epithermal neutrons is polarized by transmission through liquid hydrogen in which the nucleon spins are aligned along the beam direction. This polarized neutron beam is then scattered on various nuclear targets, and the difference of the cross section for both incoming helicity states is measured. Any helicity-dependent difference is evidence for parity violation in the neutron-nucleus interaction. In heavy nuclei, a number of factors enhances the magnitude of the effect over that expected in lighter systems. Interference between $s$ states that are dominant and $\mathrm{p}$ states in which parity may be violated gives an enhancement due to the fact that an interference term is large, as well as a gain in amplitude from s-state involvement. This experiment has observed parity violation in a number of nuclei and has noted that the sign of the effect is predominantly positive. This asymmetry is surprising in view of the belief that the resonant states should be distributed randomly, and a reassessment of the traditional view is underway. 
Medium Energy Physics Division

Los Alamos, New Mexico 87545

Title of Project: Physics with Muons at LAMPF

Person in Charge: $\quad$ P. D. Barnes

Telephone: $\quad$ (505) 667-2000

Scientific Staff: $\quad$ J. Bradbury, M. Paciotti, O. van Dyck, M. Leon, M. Schillaci, H. White

FY 1992 Funding: $\quad \$ 400,000$

In addition to the rare-decay program, two important experiments involving six institutions are in progress at LAMPF, which make use of the intense beams of low energy muons available there. The physics topics being addressed with muons include (1) determination of weak hadronic form factors and (2) precision tests of quantum electrodynamics (QED).

Measuring the recoil asymmetry in negative muon capture on ${ }^{3} \mathrm{He}$, resulting in a triton and a neutrino, provides a measure of the poorly known induced pseudoscalar form factor $g_{p}$. Muonic helium is formed and then polarized in its ground state with surprisingly high average polarizations of about 25 percent, including both vector and tensor polarized muonic helium. The next phase of the experiment involves detecting the recoil triton and its direction.

An ultrahigh precision measurement is being assembled to determine the hyperfine structure interval in muonium to $10 \mathrm{ppb}$ and the magnetic momezt ratio $\mu_{\mu} / \mu_{\mathrm{p}}$ to $50 \mathrm{ppb}$. This represents factors of 5 to 10 improvement over present values and an extremely stringent test of QED. The new experiment uses a high field solenoid with excellent field homogeneity and chopped muon beam to provide "old muonium" for narrowing of the microwave resonance.

For the future, a proposed pulsed lepton source will utilize an upgraded,LAMPF proton storage ring to produce intense pulsed $(0.25-\mathrm{msec})$ beams of muons and neutrinos. The advantages of such beams include freedom from short-lived backgrounds, line-width narrowing, a precise zero in time, and high efficiency for coincidence experiments involving pulsed lasers. The physics accessible with pulsed muons includes a more precise determination of the muon lifetime, improved measurements of the muonium hyperfine interval, and new studies of muon capture and the spectroscopy of exotic atoms. 


\section{LOS ALAMOS NATIONAL LABORATORY}

Isotope and Nuclear Chemistry Division

Los Alamos, New Mexico 87545

Title of Project: $\quad$ Mass and Decay Measurements of Neutron-Rich Nuclei

Person in Charge: $\quad$ D. J. Vieira, J. M. Wouters

Telephone: $\quad(505) 667-7231$

Scientific Staff: $\quad$ G. W. Butler

FY 1992 Funding: $\quad \$ 647,(0) 0$

This program is directed towards the characterization and understanding of nuclei that lic far from the normal beta-stable nuclei. We are interested in learning how the nuclear structure of these nuclei changes with the addition of neutrons. In the extreme limit of particle binding, that is, for nuclei that lie close to the neutron drip line, the presence of a neutron-rich skin or "halo" gives us the opportunity to investigate the transition from normal nuclear matter to pure neutron matter as it is postulated to exist in astrophysical objects like neutron stars.

This research is carried out on a recoil spectrometer, called the Time-of-Flight Isochronous (TOFI) Spectrometer, whose unique isochronous operating principle enables the direct mass measurement of a wide variety of reaction products. To date, more than 80 mass measurements extending from ${ }^{11} \mathrm{Li}$ to ${ }^{6 t} \mathrm{Fe}$ have heen reported by the TOFI collaboration. Highlights of this research include (1) the mass measurement of neutron drip line nuclei, such as ${ }^{11} \mathrm{Li},{ }^{14} \mathrm{Be},{ }^{17} \mathrm{~B}$, where binding energetic is the dominant factor in determining how far the neutron halo extends; (2) the revelation and confirmation of strong two-body shell model interactions leading to unexpected hinding energy systematics in the $\mathrm{N}=14-16$ isotones of $\mathrm{O}, \mathrm{F}$, and $\mathrm{Ne}$; (3) the localization of the deformed intruder state region around

${ }^{31} \mathrm{Na}$ to its nearest neighbors, dramatically illustrating the collapse of the $\mathrm{N}=20$ shell gap in these nuclei; and (4) the investigation of the isospin dependence of neutron and proton pairing energies in the fp shell. Beyond these mass measurements, the TOFI Spectrometer has also been used to "A- and Z-tag" exotic recoils for heta-delayed neutron or multineutron emission studies. First results of this work have recently heen published.

In the future, this program will complete several approved TOFI experiments, investigate the natural extension of the TOFI Spectrometer idea to a multiturn isochronous system, and undertake an aggressive research \& development (R\&D) effort in radioactive heam (RB) research. In a collaboration with German scientists, we will explore the retuning of the Experimental Storage Ring in Darmstadt as a multiturn mass spectrometer. Should this effort prove successful, the mass measurements of several radioactive beam species and possibly their inter-ring reaction products will be undertaken. Finally, as the interest in RB research increases, we have been and continue to he involved in advancing a broad range, high intensity RB facility called the Isospin Laboratory. Directly related to this new initiative is our plan to investigate the thin-target, He-jet option for the production of radioactive heams using the high intensity (1-mA) proton heam of LAMPF. 
Physics Division

Los Alamos, New Mexico 87545

Title of Project: $\quad$ Research Activity Away from Los Alamos

Person in Charge: W. W. Kinnison

Telephone: $\quad(5 u), 667-4504$

Scientific Siaff: D. Fields, B. Jacak, M. Murray, M. Sarabura, J. Simon-Gillo, I. Sullivan, H. van Hecke (NA -44); T. Carey, D. Jansen, M. Leitch, J. Moss, P. McGaughey, J-C'. Peng (Fermilah E772/E789)

FY 1992 Funding: $\quad \$ 2,350,000$

The Medium Energy Physics Group of the Physics Division at Los Alamos plays a leading role in four large collaborative experiments conducted at other facilities. CERN NA-44 studies the space/time evolution of relativistic heavy-ion coilisions by measuring inclusive particle production and twoparticle correlations. Fermilab E772 has measured the nuclear target dependence of lepton-pair production from proton berms on varions nuclear targets to understand how the quark and gluon structure in nuclei differs from that of free nucleons, and the follow-on'experiment (Fermilab E789) extends those studies by investigating two-body decays of mesons and baryons containing the bottom quark. Most recently, both the NA-44 and E772/E789 subgroups are taking a major role in defining the research program and design of the PHENIX detector that will be a part of RHIC being built at Brookhaven.

At CERN, th: first data runs of the NA-44 experiment are completed. The preliminary data on twoparticle correlations (the hest ever measured) and inclusive particle production have been presented at three major conferences, and the first papers are in progress. The experiment is heing upgraded to prepare for relativistic lead-nucleus collisions.

Fermilab E789, which is a follow-on to our highly successful Fermilab E772 experiment, is now analyzing data. Those data will lead to important results on the nuclear dependence of D- and Bmeson production cross sections. Additionally, new data on the A-dependence of charm production at negative $x_{F}$, as well as very large $x_{F}$, were obtained. Papers will be published in the near future.

Finally, members of P-2 play a major role in both the physics and detector definition of the PHENIX collaboration for RHIC. Individuals involved in the project have made the physics case for the dimuon measurements and are involved in helping to define that part of the experiment. Others have worked on the silicon vertex measurement requirements for the main detector, and both groups are involved in contributing to the Conceptual Design Report for the PHENIX detector. 
Physics Division

Los Alamos, New Mexico 87545

Title of Project: Solar Neutrino Studies

Persons in Charge: R. G. H. Robertson, J. F. Wilhelmy

Telephone:

(505) 667-1346

Scientific Staff: $\quad$ T. J. Bowles, P. J. Doe, M. A. Fowler, A. Hime, H. A. O'Brien, J. S. Nico, D. Vieira, J. Wouters, J. F. Wilkerson

FY 1992 Funding: $\quad \$ 1,300,000$

One of the outstanding problems in modern physics is the "solar neutrino problem," in which less than half the calculated flux of high energy neutrinos from the Sun is observed. The cause may be some fundamental misunderstanding of solar physics, or it may be an indication of new physics beyond the Standa.d Model, specifically nonzero neutrino mass. At present, the uncertainties in modeling the high energy neutrinos make it mandatory to carry out other experiments.

Our program to resolve this question includes collaborations with the Russian Academy of Sciences on $a^{71} \mathrm{Ga}$ radiochemical measurement Soviet-American Gallium Experiment (SAGE) of the low energy $\mathrm{p}$ $p$ neutrino flux and participation in the Sudbury Neutrino Observatory (SNO) to measure the spectrum and flavor composition of the high energy neutrinos.

SAGE has recently reported results that the flux is about half of that calculated. Although the precision is not sufficient to completely rule out solar explanations, the result is most consistent with an explanation involving neutrinos oscillating from one type to another. If more data and a full-scale test of the experiment employing an artificial neutrino source confirm the early indications, SAGE will strongly favor an explanation involving new physics.

A truly conclusive demonstration of new physics requires that some new phenomena be observed. SNO may provide this, as it can observe all three known types of neutrinos. As only one type (electron) neutrino can he produced in the Sun, ohservation of the other types (muon or tau) would provide "the smoking gun" for new physics. SNO is now under construction and expects to hegin data collection in 1996. After the first year of operation, the origin of "the solar neutrino problem" should hecome crystal clear. 


\section{LOS ALAMOS NATIONAL LABORATORY}

Physics Division

Los Alamos, New Mexico 87545

Title of Project: Nuclear Level Densities

Person in Charge: $\quad$ R. C. Haight

Telephone: $\quad$ (505) 667-2829

Scientific Staff: $\quad$ S. M. Sterbenz, F. Bateman, T. Lee

FY 1992 Funding: $\quad \$ 216,000$

Nuclear level densities are being studied through proton and alpha-particle emission spectra from neutron-induced reactions. With the "white" neutron source at Weapons Neutron Research/LAMPF, these measurements can be made with neutrons from threshold to $50 \mathrm{MeV}$ concurrently. Derivation of the nuclear level densities is based on the assumption of a compound-nuclear, statistical reaction mechanism. From data over this wide energy range, other mechanisms such as precompound particle emission, which increases in importance with increasing incident energy, can be identified, quantified, and corrected for. This year, we are completing analysis of data on (n,alpha) reactions on ${ }^{27} \mathrm{Al}$ and ${ }^{56} \mathrm{Fe}$ and have just completed taking data on the ${ }^{59} \mathrm{Co}(\mathrm{n}$,alpha) reaction. With the arrival of thick CsI(Tl) scintillators, we are developing a capability of measuring proton emission spectra up to $50 \mathrm{MeV}$. 
Title of Project: Physics of Production and Transmutation of Nuclei

Person in Charge: $\quad$ D. D. Strottman

Telephone: $\quad$ (505) 667-7742

Scientific Staff: $\quad$ M. Bozoian, T. R. England, G. M. Hale, D. G. Madland, J. R. Nix, A. J. Sierk, P. G. Young

FY 1992 Funding: $\quad \$ 410,000$

A wide variety of theoretical research on nuclear reactions and structure is undertaken by the Nuclear Theory and Applications Group, T-2. The work of the group provides an extensive and reliable nuclear physics database for low- and medium-energy scattering, as well as for improved nuclear structure models. A wide variety of national programs (accelerators, reactors, waste management, nuciear medicine, and defense) relies heavily on such a nuclear database. In addition, an effort has been initiated in constructing a library of medium energy pion-, proton-, and neutron-induced reaction data.

The analysis of few-hody reactions is indispensable for many national programs. In addition, they often provide dividends in unanticipated results. Recently, we have obtained level structures for several of the light systems that differ significantly from the "accepted" schemes. These new level structures account for previously unexplained features in the experimental data (including those for muon-catalyzed fusion reactions) and can be used to provide relatively simple time-dependent descriptions of complex nuclear-scattering processes. We have also developed an R-matrix-hased method to derive scattering properties from a nuclear structure (shell model) calculation, and we are applying the method to study the "neutron halo" effects in "Li.

We continue improvement of statistical, pre-equilibrium, and direct reaction theories and parameter systematics for better evaluation, interpolation, extrapolation, and prediction of nuclear data. We are currently developing a multistep compound/multistep direct reaction model that will he utilized for both basic and applied physics.

Recent advances in the understanding of the fission process are being applied to detailed model calculations of particle-emission properties and fission-fragment distributions. The evaluation of fission product yields for the national database, augmented with model values, is our responsibility. In addition, we have the responsibility for 21 other nuclides.

Work is continuing on the understanding of medium energy proton-nucleus and neutron-nucleus cross sections. A global medium energy nucleon-nucleus Dirac optical potential has heen developed for ${ }^{208} \mathrm{~Pb}$. Predictions have heen made for unmeasured medium energy neutron scattering observables that are now heing measured at Weapons Neutron Research.

We are continuing our nuclear structure studies to obtain better descriptions of ground-state and excited-state properties, both near and far from the line of stahility. Applications include hadronic 
scattering processes, exotic nuclei that can be reached using radioactive heams, and nuclear astrophysics. Both relativistic and nonrelativistic models are being developed that provide information out to the extremes in isospin, atomic number, deformation, spin, and single-particle orbits. We currently calculate hulk nuclear properties and properties of individual states and expect to calculate transitions between states and the discrete level density of states. 
Theoretical Division

Los Alamos, New Mexico 87545

Title of Project: Medium Energy Theory

Person in Charge: J. T. Goldman

Telephone: (505) 667-3244

Scientific Staff: $\quad$ J. L. Friar, B. F. Gibson, J. N. Ginocchio, P. Herczeg, R. R. Silbar

FY 1992 Funding: $\quad \$ 1,105,000$

Theoretical research on a broad range of topics, providing guidance for experimental work at medium energy physics facilities and involving all four of the fundamental interactions, is undertaken by the Medium Energy Physics Theory Group, T-5.

Studies of antiproton reactions with nuclei probe the basic strong interaction between matter and antimatter. Investigations of the scattering of mesons and nucleons from nuclei attempt to elucidate the mechanisms that drive these reactions and to probe the structure of complex nuclei. Recent calculations have demonstrated that nuclear structure plays an important role in reactions that change the charge of scattered pions by two units.

An extensive program at the forefront of few-hody nuclear physics is being pursued. This program relates the $\mathrm{NN}$ interaction to a wide variety of observables in light nuclei. New calculations have decomposed the trinucleon and a-particle potential energies from several potential models into their component parts, demonstrating that the one-pion-exchange potential is the single most important force component in light systems. Green's function Monte Carlo calculations of the $A=5$ system have been performed. In addition, new Monte Carlo methods to calculate dynamic properties of quantum systems have been developed and applied to the longitudinal response function obtained in electronscattering experiments.

Exciting new prospects of describing nucleons and nuclei in terms of underlying quark degrees of freedom are heing pursued, using models in which physical nucleons are either solitons or clusters of quarks. Recent progress includes predictions of exotic mesons and dibaryons. A particularly likely dibaryon may he accessible to experiment at LAMPF.

Theoretical studies of weak interaction processes are confronted by polarized proton-scattering data from LAMPF. The information on time reversal violating interactions that could he obtained from neutron transmission experiments is being investigated. The manifestations of possible new interactions that appear in extensions of the Standard Model are studied in rare and forbidden particle decays, in conjunction with experiments at LAMPF.

Renormalization effects in the quantum theory of gravity are being applied to large-scale cosmology. The upper bound on the baryonic matter density in the universe, derived from nucleosynthesis, is not consistent with the total matter density observed dynamically. We have found that Newton's "constant" may increase at large scales, due to quantum effects, sufficiently rapidly to explain this discrepancy without introducing new types of nonbaryonic stable matter, which has also been proposed 
to resolve the problem. We are extending our studics (1) similar effects on the cosmological constant and to details in galactic rotation curves.

The extensive numerical calculations involved in much of our nuclear theory research require significant use of supercomputers. 


\section{NATIONAL INSTITUTE OF STANDARDS AND TECHNOLOG Y}

lonizing Radiation Division

Gaithersburg, Maryland 20899

Title of Project: Ahsolute Neutron Measurements

Person in Charge: G. L. Greenc

Telephone:

(301) $975-4842$

Scientific Staff: $\quad$ M. S. Dewey, D. Gilliam, J. M. Snow

FY 1992 Funding: $\$ \$ 130,(0) 0$

The beta-decay lifetime of the free neutron is an important parameter in the theory of weak interactions, in astrophysics and in cosmology. Direct determinations can be compared with indirect estimations (based on measured values of the heta asymmetry in neutron decay) to give a sensitive test of the Standard Model for weak interactions and to provide a sensitive probe for the possible existence of right-handed currents. In cosmology, the value of the neutron lifetime, in conjunction with observed universal abundance of light elements, can he used as a very rigorous test of Big Bang nucleosynthesis as well as on the possible number of light neutrino species. In addition, the weak matrix elements for neutron decay are important in a variety of astrophysical contexts including the standard solar model and supernova core collapse.

The National Institute of Standards and Technology (NIST) "in heam" neutron lifetime experiment requires two ahsolute measurements. The first measurement concerns the determination of the decay rate of neutrons in flight in a low energy neutron beam. The second measurement concerns the determination of the flux in that heam. Significant progress has heen made in hoth areas. A totally re-engineered decay detector has been installed on the floor of the NIST Cold Neutron Research Facility in Gaithershurg. With a number of new features incorporated into its design and with extended running time, we expect to obtain a significant improvement in this phase of the measurement.

Over a period of approximately 6 months in 1992, intercomparisons among several new methods for the absolute determination of neutron fluxes were carried out at NIST. Preliminary analysis of this data indicates the possibility of neutron flux determinations with an accuracy approaching the level of 0.1 percent. Such measurement would he important, not only for the neutron lifetime determination hut also for the determination of low energy neutron cross sections for nuclei of importance in nuclear physics and nuclear technology. 


\section{NATIONAL INSTITUTE OF STANDARDS AND TECHNOLOG Y}

Physics Laboratory, lonizing Radiation Division

Gaithersburg, Maryland 20899

Title of Project: $\quad$ Neutron Cross Section Standards and Instrumentation

Persons in Charge: R. S. Caswell, O. A. Wasson

Telephone: (301) 975-5567

Scientific Staff: $\quad$ A. D. Carlson, R. A. Schrack, J. A. Grundl

FY 1992 Funding: $\quad \$ 475,000$

The primary goals of the neutron cross section standards program are to provide the foundation of the neutron nuclear data system and to develop new and improved methods for detecting the neutron. This activity inciudes both an extensive experimental effort as well as coordination of the national evaluation of the neutron cross section standards for the U.S. These standards form the hasis for nearly all neutron nuclear data, since these data are measured relative to the standards. An improvement in the accuracy of the standards will improve the accuracy of the entire database without an extensive remeasurement effort. These data are also of strong interest to nuclear science and have important applications in technology, such as nuclear safety, nuclear medicine, nuclear fusion, mineral exploration, radiation therapy, electric power generation, and national defense.

Measurement activities in this program have concentrated on the reduction and understanding of the systematic errors that limit the accuracy of the important neutron reaction standards, namely fission cross sections for uranium and neutron interactions in boron and lithium. The measurements cover a wide range of neutron energies and use an extensive array of neutron sources and detectors, all to improve accuracy by means of redundant measurements.

Measurements to extend the use of the boron standard to higher neutron energies are in progress using the intense pulsed neutron source at ORNL. The neutron detectors developed and calibrated at National Institute of Standards and Technology serve an essential element in improving this standard. The database for the neutron-induced fission reactions in a few selected actinide isotopes is being extended in measurements at the intense neutron source at LANL. Neutron-induced charged-particle spectral measurements important for medical applications are also in progress at this facility. These measurements will continue into next year. 
Physics Division

Oak Ridge, Tennessee 37831

Title of Project: The Holifield Heavy-Ion Research Facility/RIB Facility

Persons in Charge: C. M. Jones, R. L. Robinson

Telephone:

(615) 574-4109

Scientific Staff: $\quad$ G. D. Alton, R. L. Auble, W. T. Milner, D. K. Olsen

FY 1992 Funding: $\$ \$ 3,580,000$

HHIRF serves as a national user facility for research in heavy-ion nuclear physics. Typically, 150 researchers participate in experiments each year. The facility includes a $25-\mathrm{MV}$ folded tandem accelerator and the Oak Ridge Isochronous Cyclotron (ORIC), which can serve as a booster accelerator. The tandem accelerator is the world's highest voltage electrostatic accelerator. The tandem accelerator and ORIC, operating separately or as a combined system, provide the widest range of projectile species with precision energies of any facility. Parameters of the facility are: mass range, 1-238 AMU; energy range for light ions, 1-25 MeV/AMU; energy range for the heaviest ions, 1-6 MeV/AMU; intensity, $10^{11}-10^{13}$ particles per second; pulse width, dc to 1 nanosecond; and energy resolution, $0.01-0.3 \%$.

Major experimental equipment at the Holifield facility includes a magnetic spectrometer; a velocity filter; an array of $\mathrm{BaF}_{2}$ detectors; a 1.6-m-diameter scattering chamber; and a 72-element, 4-pi solid angle Nal gamma-ray detector array which surrounds a 33-cm-diameter spherical chamber (the Spin Spectrometer). Up to 20 of the Spin Spectrometer elements are routinely replaced with Comptonsuppressed high resolution gamma-ray Ge detectors. These detectors are also used alone in a closepacked configuration. The University Isotope Separator Oak Ridge magnetic isotope separator project with a nuclear orientation facility is located at HHIRF. These experimental devices are complemented by a powerful and versatile data acquisition and analysis system.

Adjoining HHIRF are buildings for the Joint Institute for Heavy-Ion Research, a joint venture of ORNL, the University of Tennessee, and Vanderbilt University. The mission of the Institute is to enhance the scientific environment of HHIRF principally through support of workshops and guests. In addition, it provides living accommodations for HHIRF users.

The Holifield facility has been funded to reconfigure the accelerator system to produce radioactive ion heams, with atomic masses up to 80 and beam currents and energies sufficient to undertake research in the areas of nuclear structure and astrophysics. This reconfiguration will be complemented by the construction of a recoil mass spectrometer. These projects and associated target areas and beam lines are scheduled for completion in early 1995 . As this conversion progresses, there will be no operation for users in 1993 and 1994. 


\section{OAK RIDGE NATIONAL LABORATORY}

Physics Division

Oak Ridge, Tennessee 37831

Title of Project: Heavy-Ion Nuclear Research

Persons in Charge: F. E. Bertrand, J. R. Beene, J. D. Garrett, D. Shapira

Telephone:

(615) 574-4737

Scientific Staft: $\quad$ C. Baktash, E. Chavez, D. Cullen, J. Gomez del Campo, M. L. Halbert, N. R. Johnson, M. Korolija, I. Y. Lee, K. S. Toth, P. H. Stelson, D. F. Winchell

FY 1992 Funding: $\quad \$ 2,350,000$

One of the primary goals of heavy-ion nuclear physics is to understand the structure of the nucleus under extreme conditions of temperature, excitation energy, and rotational frequency. This research program addresses these goals through studies of nuclear structure, reaction spectroscopy, and nuclear-collision dynamics. Results from this research have a strong intrinsic interest to nuclear science and have growing importance to many other areas of science, a prime example being astrophysics and cosmology.

The main thrust of the nuclear structure program is to establish the single-particle basis of nuclear correlations for nuclei with large amounts of angular momentum and excitation energy and at the extremes of isospin. Discrete livel spectroscopy, measurements of electromagnetic transition rates, studies of the continuum of nuclear states, studies of nuclei far from stability, and systematic analyses of such data are involved in this work. Recent results from these studies include the systematic observation of identical low spin bands in neighboring nuclei and studies of the lifetime and decay of superdeformed states. The future research program in this area of physics will be centered on proton-rich radioactive beams from the accelerator upgrade now in progress at ORNL.

Heavy-ion nuclear reactions exhibit a richness of phenomena in which reaction mechanism effects and spectroscopic properties of the reactants can be coupled in interesting and intricate ways. Investigations therefore focus on both the reaction mechanisms and on the use of heavy-ion reactions to obtain information on nuclear excitations. Work in this research area utilizes a newly commissioned array of $76 \mathrm{BaF}_{2}$ detectors as well as the ORNL Spin Spectrometer. The Spin Spectrometer allows the impact parameter dependence of nuclear reactions to be studied, providing, for example, new insights into reactions at near and subbarrier energies.

Studies of nuclear collision dynamics emphasize gross properties of nuclei and utilize reactions ranging from peripheral quasi-elastic, through deep inelastic to fusionlike processes. Exclusive measurements of charged particles, ranging in mass from individual nucleons to that of the composite system, emitted in nuclear reactions are used in these studies. Investigations are implemented with a state-of-the-art detector system, Heavy-Ion Light Ion, which has a large solid angle, is highly segmented, and has a logarithmic response. In recent studies of $15 \mathrm{MeV} /$ nucleon $\mathrm{Ni}+\mathrm{Ni}$ reactions, the average lifetime for proton emission was measured. 
Physics Division

Oak Ridge, Tennessee 37831

Title of Project: Nuclear Physics with Light Ions and Neutrons

Persons in Charge: F. E. Bertrand, J. R. Beene, S. Raman

Telephone:

(615) 574-4737

Scientific Staff: $\quad$ R. L. Auble, D. J. Horen, P. Mueller

FY 1992 Funding: $\quad \$ 1,280,000$

The research performed under this program is designed to study the fundamental modes of nuclear excitation. The major effort of this research program is the study of the most collective of nuclear excitations: giant multipole resonances. This program is also concerned with the study of neutron .scattering and capture reactions. Low energy neutron-scattering measurements on selected nuclides provide a unique contribution to the study of fundamental excitations.

For the last several years, the primary effort of this research has been focused on the study of properties of giant resonance strength excited by the Coulomb field in intermediate energy heavy-ion reactions. Based on our construction of a large array of $\mathrm{BaF}_{2}$ detectors, we have developed powerful particle- $\gamma$ and particle- $\gamma \gamma$ coincidence techniques and the experimental hardware to exploit them. One of our recent efforts has involved use of $95 \mathrm{MeV} /$ nucleon ${ }^{36} \mathrm{Ar}$ and $60 \mathrm{MeV} /$ nucleon ${ }^{86} \mathrm{Kr}$ heams to excite, isolate, and identify the two-phonon giant dipole resonance using $\gamma$-ray coincidence techniques. Further experiments on two-phonon states based on Giant dipole resonance (GDR) will be carried out in early FY 1993.

In the neutron capture program, we have developed a computer code to calculate a direct-reaction capture cross section and to split it into valence and potential components. Extensive slow-neutron capture data on all the stable sulfur, magnesium, silicon, and calcium isotopes have been successfully analyzed with this code. The level schemes resulting from neutron capture gamma-ray studies are generally complete (for a particular spin window) up to an excitation energy of 3 to $4 \mathrm{MeV}$. When combined with the results from other measurements, the resulting complete level schemes can he subjected to an analysis of nearest-neighbor spacing distributions that can give a clue as to a possible transition from regular to chantic behavior. We have carried out such an analysis in ${ }^{116} \mathrm{Sn}$. 
Oak Ridge, Tennessee 37831

Title of Project: High Energy Heavy-Ion Reactions

Persons in Charge: F. Plasil, G. R. Young

Telephone: (615) 574-4711

Scientific Staff: $\quad$ T. C. Awes, H. J. Kim, F. E. Obenshain, S. Saini, S. P. Sorensen, P. Stankus

FY 1992 Funding: $\$ \$ 1,050,000$

QCD lattice calculations predict that, at sufficiently high energy densities, hadronic matter undergoes a transition to a new phase of matter-the quark-gluon plasma-in which quarks and gluons are deconfined over a relatively large volume. Collisions between heavy nuclei at ultrarelativistic energies are likely to produce the energy densities, estimated to be greater than $2-3 \mathrm{GeV} / \mathrm{fm}^{3}$, necessary for this phase transition to occur. The purposes of this project are (1) to study the conditions required for producing this fundamental state of matter and (2) to study its properties.

The ORNL group is a member of the WA93 Collaboration at CERN in Geneva, Switzerland, where ${ }^{16} \mathrm{O}$ and ${ }^{32} \mathrm{~S}$ nuclei are accelerated to energies of $200 \mathrm{GeV} /$ nucleon in the Super Proton Synchrotron accelerator. Although the primary goal of the collaboration is the search for indications of the quark-gluon plasma formation 'by means of direct photon measurements, other goals are (1) to carry out a survey of collisions hetween nuclei at ultrarelativistic energies; (2) to compare the results with those obtained with protons under the same conditions; and (3) to determine the extent of attained energy densities and of nuclear stopping. ORNL is responsible for the design, construction, and operation of the calorimeters associated with the WA93 experiment.

The ORNL group plays a leadership role in the PHENIX Collaboration, which is designing one of the two major experiments for the BNL RHIC. PHENIX emphasizes the measurement of photons, electron and muon pairs, and selected aspects of hadron emission. Leptons and photons, due to their penetration properties, are believed to be the ideal probes of the quark-gluon plasma. Lepton pairs also result from vector meson decays, whose properties are believed to be a good signal of creation of dense matter and deconfinement.

At CERN, the ORNL group has joined the WA98 Collaboration, which plans to make use of very heavy projectiles such as lead. Lead beams are scheduled to be available in early 1994. To pursue the goals outlined previously, WA98 intends to measure direct photon yields and kaons, pions, and neutral mesons associated with individual events. 
Physics Division

Oak Ridge, Tennessee 37831

Title of Project: Theoretical Nuclear Physics

Person in Charge: $\quad$ M. R. Strayer

Telephone:

(615) $574-4590$

Scientific Staff: $\quad$ F. E. Barnes, K. T. R. Davies, G. Gatoff, J. Liu, G. R. Satchler, C. Y. Wong

FY 1992 Funding: $\$ \$ 45,000$

The physics program in nuclear theory comprises research in relativistic heavy-ion collisions, hadron spectroscopy, low energy heavy-ion reaction mechanisms, and nuclear astrophysics. The program is strongly tied to the experimental research in the ORNL Physics Division. Part of the relativistic program is directly involved with analyzing relativistic heavy-ion data from CERN, and with collaborations at BNL on relativistic heavy-ion physics. The hadron spectroscopy program relates to the programs at CEBAF, Newport News, Virginia, and LAMPF, Los Alamos, New Mexico.

Research topics include pair production from relativistic heavy-ion collisions, color hydrodynamics, string-parton models of hadronic evolution and hadronization, quark models of meson structure, relativistic theory of nuclear matter, low energy direct reactions, Hartree-Fock studies of nuclear structure, models of nonlinear collective motion and chaos, lattice techniques for classical field theories, and numerical simulations of type II supernovae explosions.

The Center for Computationally Intensive Physics has been established in the Physics Division and partially supports the nuclear theory activities. It is part of a consortium that will have access to the Intel Paragon supercomputer, a unique resource for theoretical nuclear physics, and is interdisciplinary in scope, with collaborations in atomic, nuclear, particle, and condensed matter physics. 
UNISOR (University Isotope Separator-Oak Ridge)

Oak Ridge, Tennessee 37831

Title of Project: $\quad$ UNISOR

Porson in Charge: $\quad$ H. K. Carter

Telephone: $\quad$ (615) 576-2642

Sciontific Staft: $\quad$ B. R. Bengtsson, P. F. Mantica

FY 1992 Funding: $\$ 475,000$

Fundamental experimental nuclear physics research is carried out on nuclei with extreme numbers of neutrons and protons to test nuclear theories at relatively low energy and spin. The experimental capabilities at the University Isotope Separator at Oak Ridge (UNISOR) are unique in the U.S. in their ability to measure certain nuclear properties such as moments, spins, changes of nuclear radii of ground states of very short-lived, rare species as well as the mixing ratios of transitions and lifetimes of excited states of these nuclei.

Current research ir... numbers especially in the platinum and lead regions. In the Te and Xe nuclides, measurements are carried out to characterize the "intruder" structures. Using the nuclear orientation facility and detailed electron and gamma-ray spectroscopy, coexisting $\mathrm{K}^{\boldsymbol{\pi}}=0^{+}$and $\mathrm{K}^{\boldsymbol{\pi}}=2^{+}$, bands at low excitation energy have been found in ${ }^{184} \mathrm{Pt}$. Isotope shifts, and hyperfine structure has been extended to ${ }^{186} \mathrm{~T} 7$ in a search for a correlation between the $I=1 / 2$ to $9 / 2$ energy difference systematics as well as systematics of proton, neutron configurations on possible deformation effects.

The emphasis of the UNISOR research program is shifting from the study of nuclei produced by stable beams on stable targets to those that can only be produced by radioactive beams on stable targets. The UNISOR on-site staff will work full-time on developing techniques to produce radioactive ion beams using the ORNL accelerators. The UNISOR university researchers will work on developing is necessary experimental capabilities to use these beams effectively, especially the Recoil Mass Spectrometer. 


\section{OAK RIDGE NATIONAL LABORATORY}

Engineering Physics and Mathematics Division

Oak Ridge, Tennessee 37831

Title of Project: $\quad$ Oak Ridge Electron Linear Accelerator (ORELA)

Person in Charge: J. A. Harvey

Telephone:

(615) $574-4591$

Scientific Staff: $\quad$ J. G. Cravien, T. A. Lewis, D. E. Pierce

FY 1992 Funding: $\quad \$ 1,500,000$

The Oak Ridge Electron Linear Accelerator (ORELA) is an intense pulsed neutron source for studying the interaction of neutrons with nuclei at low neutron energies. Results are used for the design and operation of safe and efficient fission reactors, fusion energy issues, as well as medical and other applied needs. ORELA is used by experimenters from the Engineering Physics and Mathematics, Analytical Chemistry, Solid State and Physics Divisions of ORNL, by physicists from nearby universities, outside companies, and by other DOE-sponsored scientists.

The ORELA facility consists of a 180-MeV electron linear accelerator; neutron-producing targets; 10 evacuated, underground neutron flight tubes (as long as 200 meters) leading to detector stations; diverse particle and gamma-ray detectors; and data acquisition and analysis computer systems. The accelerator produces 20-ampere pulses of nanosecond widths with a maximum electron beam power of 60 kilowatts in a beam only $1 \mathrm{~cm}$ diameter. Measurements of the interactions of neutrons with nuclei up to tens of $\mathrm{MeV}$, in some sases with a neutron energy resolution up to one part in 4,000.

Six new personal-computer-based data acquisition systems have been implemented, utilizing in-house designed interfaces and software. They allow a much higher rate of data acquisition and increased flexibility in experiment design.

Minimal changes were required to bring the facility in compliance with current regulatory standards. Based on an in-depth analysis, ORELA achieved a low hazard classification. The upgrade program continues to maintain excellent accelerator reliability and availability. Current cost reduction measures include upgrading modulators to use lower cost thyratrons, oil reconditioning and reuse, and selection of materials to minimize Resource Conservation Recovery Act waste. 
Engineering Physics and Mathematics Division

Oak Ridge, Tennessee 37831

Title of Project: $\quad$ Neutron Reactions-Nuclear Data

Person in Charge: $\quad$ D. C. Larson

Telephone: $\quad$ (615) 574-6119

Scientific Staff: $\quad$ J. K. Dickens, C. Y. Fu, R. Gwin, J. A. Harvey, D. M. Hetrick, N. W. Hill, N. M. Larson, R. W. Peelle, J. H. Todd, L. W. Weston

FY 1992 Funding: $\quad \$ 3,780,000$

The primary purposes of Oak Ridge Electron Linear Accelerator (ORELA) research are twofold: (1) to measure, analyze, interpret, and parameterize neutron cross sections for better understanding of basic nuclear physics and (2) to provide cross section data for neutron-induced reactions for materials, reactions, and energy ranges of importance to applied programs, including fission and fusion reactors, nuclear medicine, space applications, and nuclear waste management.

No nuclear models at present can predict cross sections in the resonance energy region; thus, much of our work focuses on this important region. Taking advantage of the high intensity and excellent energy resolution of ORELA, researchers measure total, capture, fission and differential scattering cross sections to provide information on energies, widths, spins, and parities of resonances. A state-ofthe-art resonance parameter code developed at ORELA is used for the data analyses, results of which are fundamental to both basic and applied research. Recent results include measurement and analysis of resonances in ${ }^{56} \mathrm{Fe}$ (thermal to $800 \mathrm{keV}$ ), ${ }^{59} \mathrm{Co}$ (thermal to $100 \mathrm{keV}$ ), and ${ }^{239} \mathrm{Pu}(0.01 \mathrm{eV}$ to $10 \mathrm{keV}$ ).

At higher energies, measurements of neutron transmission, total inelastic, capture, fission, and neutron and gamma-ray emission cross sections are performed to henchmark nuclear model code development and meet needs of engineers and scientists who need nuclear information in their work. Current measurements are involved with (1) resolving a long-standing problem in reactor physics, the shape of the cal ture-to-fission ratio in ${ }^{235} \mathrm{U}$ at low neutron energies; (2) measurements of the neutron emission double differential cross sections for efficient design of fusion reactors; (3) measurements of poorly known nitrogen cross sections needed for analysis of Hiroshima/Nagasaki bomb data used in determining allowable doses to radiation workers; (4) studies of DNA single hase annihilation using the ORELA positron source; and (5) hasic physics studies related to parity violation and time-reversal, phonon softening around the critical temperature in high temperature superconductors, and measurements of the neutron polarizability and charge structure. The phonon-softening and neutron polarizability measurements are hoth recipients of recent awards. 
Physics Division and Engineering Physics and Mathematics Division

Oak Ridge, Tennessee 37831

Title of Project: Nuclear Data Compilation and Evaluation

Persons in Charge: M. J. Martin, D. C. Larson

Tolephone: $\quad$ (615) 574-4691

Scientific Staff: $\quad$ Y. A. Akovali, C. Y. Fu, D. M. Hetrick, D. C. Larson, M. R. Lay, M. J. Martin, L. W. Weston

FY 1992 Funding: $\quad \$ 610,000$

The data compilation and evaluation activity is carried out by the Nuclear Data Project (NDP) (\$485,000, Physics Division) and at the Oak Ridge Linear Accelerator (ORELA) $(\$ 125,000$, Engineering Physics and Mathematics Division).

NDP is responsible for collecting and evaluating nuclear structure data for 56 mass chains in the mass region $A \geq 200$. The project coordinates its work with other evaluation centers comprising the U.S. Nuclear Data Network (USNDN). USNDN is itself part of the International Nuclear Structure and Decay Data Network.

The work involves the reading and critical analysis of all literature on nuclei in the above mass region. For each research paper, the evaluators extract the data, compare them with data from other sources, and prepare critical reviews with recommended values for a wide range of nuclear structure properties. Systematic trends in these properties are investigated both as a guide in the e aluation procedure and as a means of gaining new insight into nuclear structure phenomena.

The data evaluated by NDP are made available to the research community through publication of the journal Nuclear Data Sheets. NDP is responsible for the scientific editorship of this monthly journal.

Evaluation of neutron-induced cross sections for the U.S. Evaluated Nuclear Data File/B (ENDF/B) is done as part of the ORELA research program. Accurate neutronics calculations, needed for design and safety analyses of more economical fission and fusion reactors, nuclear medicine, space applications, and waste management, require precise evaluated cross sections and related quantities. ENDF/B-VI, released in 1991, contains new types of information to meet new demands of scientists and engineers who require evaluated data for their work, as well as improved cross section results based on new experimental data and refined nuclear models. Current work focuses on theory and model code improvement and participation in an international effort to compare results from U.S., Japanese, and European evaluated libraries and understand and resolve significant differences among them. 


\section{PACIFIC NORTHWEST LABORATORY}

Nuclear Chemistry Section

Richland, Washington 99352

Title of Project: Decay Measurements of Extremely Neutron-Rich Nuclides

Person in Charge: $\quad$ P. L. Reeder

Telephone:

(509) 376-0948

Scientific Staff: $\quad$ W. K. Hensley, H. S. Miley, R. A. Warner

FY 1992 Funding: $\$ \$ 90,000$

Lifetimes and delayed-neutron emission properties of very neutron-rich nuclides are being measured at the LAMPF accelerator at LANL and at the Tri-University Meson Facility (TRIUMF) accelerator at Vancouver, BC. Nuclides in the light mass region $(A<70)$ are being produced and measured using the Time-of-Flight Isochronous (TOFI) Spectrometer at LAMPF. Each nuclide passing through the spectrometer is identified according to its mass number, proton number, and ionic charge. Lifetime measurements consist of determining the time-interval distribution between the arrival of a specific nuclide and the subsequent emission of a neutron. Delayed-neutron emission probabilities $\left(P_{n}\right)$ are calculated from the intercept of the time-interval distribution curve, the number of identified ions, the half-life, and the neutron counting efficiency.

Previous work resulted in the remeasurement of the half-lives of 16 nuclides in the region from ${ }^{9} \mathrm{Li}$ to ${ }^{35} \mathrm{Al}$ and the first measurements of the half-lives of ${ }^{25} \mathrm{~F}$ and ${ }^{28} \mathrm{Ne}$. Emission probabilities of 19 nuclides were determined including the first values for ${ }^{12} \mathrm{Be},{ }^{14} \mathrm{~B},{ }^{17} \mathrm{C},{ }^{18} \mathrm{~N},{ }^{25} \mathrm{~F}$, and ${ }^{28} \mathrm{Ne}$. Upper limits were set for emission probabilities of 12 other nuclides. A technique has been developed to determine the average energy of the delayed-neutron spectrum based on the ratio of neutron counts in different rings of neutron counter tubes embedded in the polyethylene moderator. This technique has provided the first measurement of the average energy of delayed neutrons from ${ }^{19} \mathrm{~N}$.

Experiments have been done to measure properties of nuclides in the $\mathrm{Cl}$ to $\mathrm{Mn}(\mathrm{Z}=17-25)$ region. The data are currently being analyzed. New experiments are in progress to measure probabilities for twoor three-neutron emission from nuclides in the very light mass region and to determine the average neutron energy of a large number of nuclides. Simultaneously, a search is underway for beta-delayed charged particle emission $\left({ }^{2} \mathrm{H},{ }^{3} \mathrm{H}\right.$, and $\left.{ }^{4} \mathrm{He}\right)$ from these same nuclides.

The experiments at the TRIUMF Isotope Separator On-Line (TISOL) complement the TOFI experiments by allowing detailed studies of a single nuclide. In particular, the energy spectra of delayed neutrons will be measured using a ${ }^{3} \mathrm{He}$ ionization chamber detector.

The measured half-lives, delayed-neutron emission probabilities, and average neutron energies are being compared to predictions from several models of beta decay. These data will lead to refinements in the models and thus lead to better predictions for unknown nuclides at the limits of particle stability. These experimental data are providing fundamental information needed for understanding nuclides with unusual numbers of protons and neutrons. 


\section{Research Programs at Universities}




\section{ABILENE CHRISTIAN UNIVERSITY}

\section{Department of Physics}

Abilene, Texas 79699

Title of Project: $\quad$ Measurements of Observables in the Pion-Nucleon System, Nuclear ADependence of Heavy Quark Production, and Rare Decays of D and B Mesons

Persons in Charge: M. E. Sadler, L. D. Isenhower

Telephone: $\quad$ (915) 674-2165

FY 1992 Funding: $\quad \$ 115,000$

Understanding the fundamental interactions of nature has been the quest of particle physics for more than 50 years. Although we know a great deal about the structure of nuclear matter, there are many puzzles that remain and many tools with which one may attempt to untangle the complicated picture nature presents to us. One method is to study reactions at lower to intermediate energies at accelerators such as LAMPF located at Los Alamos. Another method is to work at the highest energies possible at accelerators like Fermilab near Chicago.

The nuclear physics program at Abilene Christian University (ACU) has concentrated on work at LAMPF to study the pion-nucleon system. In the momentum interval 400 to $700 \mathrm{MeV} / \mathrm{c}$ (laboratory pion momentum), a complete set of observable quantities has been obtained. Current efforts deal with the construction of the LAMPF Neutral Meson Spectrometer (NMS) that will replace the old PI0 spectrometer. NMS already has a long list of approved experiments waiting to make use of its capabilities. ACU undergraduates have been responsible for testing the CsI calorimeter crystals along with their photomultiplier tubes.

Data have been collected at Fermilab to study the dependence of the production of heavy quarks (charm and beauty) on the type of nucleus used for the production target and to search for rare decays of the $\mathrm{D}$ and $\mathrm{B}$ mesons (containing charm and beauty quarks, respectively). This experiment involves the combined efforts of nuclear and high energy experimentalists, because the results hold considerable interest to both communities. Data analysis should be completed by mid-1993.

In 1991 and 1992, ACU has participated in an experiment to measure the eta meson production at threshold using the accelerator at the St. Petersburg Nuclear Physics Institute in Russia. Equipment purchased by DOE funds helped make this experiment possible.

Future work will likely make use of the CEBAF Large Acceptance Spectrometer as well as LAMPF's NMS. 
Title of Project: $\quad$ Study of Particle Correlations in High Energy $\mathrm{S}+\mathrm{Pb}$ and $\mathrm{Pb}+\mathrm{Pb}$ Interactions with a Magnetic-Interferometric-Emulsion Chamber

Person in Charge: $\quad$ Y. Takahashi

Telephone: $\quad$ (205) $895-6276$

Scientific Staff: $\quad$ C. H. Chan, J. G. Duthie, J. C. Gregory

FY 1992 Funding: $\quad \$ 91,000$

The atomic nucleus is the most dense form of material on Earth. The nuclear forces used to bind the nucleus are now understood to arise from the more fundamental forces of subnuclear elements, quarks and gluons, and the possibility of a new state of nulear matter, the so-called "quark-gluon plasma." This new state of nuclear matter is theoretically predicted to emerge in very high energy collisions of nuclei. Exploratory investigations, searching for a signature for such a state of matter, were made in 1986 with $200 \mathrm{GeV} / \mathrm{amu}{ }^{16} \mathrm{O}$ ions, and in 1987 and 1990 with ${ }^{32} \mathrm{~S}$ ions by using the Super Proton Synchrotron accelerator of CERN located in Geneva, Switzerland.

The University of Alabama (Huntsville) experimental nuclear group has been carrying out studies of collisions of ${ }^{16} \mathrm{O}$ and ${ }^{32} \mathrm{~S}$ nuclei and $\mathrm{Pb}$ target nuclei using emulsion films in a high magnetic field. Measurements with this system, capable of providing information on multiplicity, emission angles, charge-sign, and momentum distributions, revealed short range correlations of different charge-sign particles in excess of that expected from Bose-Einstein interference for identical bosons. The search for new states of matter is continuing in the analysis of these heavy-ion collisions. 


\section{UNIVERSITY OF ARIZONA}

Department of Physics

Tucson, Arizona 85721

Title of Project: Dynamical Behavior of Excited Hadronic Matter

Persons in Charge: P. Carruthers, I. Sarcevic

Scientific Staff: $\quad$ P. Lipa, M. Greiner, H. T. Elze

FY 1992 Funding: $\quad \$ 91,000$

The research supported by the Division of Nuclear Physics has been concentrated on the question of fluctuations and correlations in multiparticle correlations at relativistic energies. We compare the behavior of multihadron production for all projectiles and targets. Our theory group has ongoing collaborations with the experiments at UA1, NA22, NA9, and NA35 at CERN.

Previously, we pointed out that alleged scaling behavior of factorial moments (and hence of the underlying correlation functions) was in contradiction with both data and the associated mathematical identities. This analysis was in one-dimensional rapidity phase space. Presently, various groups are studying higher dimensional phase space in an attempt to see scaling.

During FY 1992, we made a number of interesting contributions. Probably the most important was to replace the famous bin-averaged factorial moment analysis with a "strip" moment which has similar use in nonlinear dynamics. The main point is that as one studies the resolution dependence, the bin method leads to spurious fluctuations that are cleaned up by an order of magnitude with our new method.

At the moment, we are looking at wavelet analysis for phenomenology, dynamics, and pattern recognition for detector design. 


\section{U NIVERSITY OF ARIZONA}

Department of Physics

Tucson, AZ 85721

Title of Project: Probing Vacuum Structure in Nuclear Collisions

Person in Charge: J. Rafelski

Telephone: (602) $621-4212$

FY 1992 Funding: $\$ 59,000$

In usual studies of physical phenomena, we de-emphasize the structure of the vacuum by considering it to be a reference state: we model physical situations by building excitations relative to it. This pragmatic approach enables one to calculate without having to resolve the problem of the internal structure of the vacuum. However, akin to any other physical state, the vacuum state possesses a number of interesting physical properties.

In a dynamical situation, such as is unavoidably encountered in laboratory experiments involving nuclear collisions, these vacuum properties can undergo a (local) rapid change and hence any approach to the study of the time and space dependence of the vacuum should, in principle, be general enough to incorporate in the description quantum transport phenomena and particle (entropy) production. These features can be studied employing the theoretical framework of (self-consistent integro-differential equations for) the Wigner function acting in the phase space and describing the time evolution of the vacuum state, in particular under changing external conditions.

Our work is based on a new seven-dimensional phase space Wigner-type nonperturbative method to study the vacuum structure, which we have recently proposed. Our objectives include the understanding of particle production and the transport properties of the vacuum, also in the classical limit. In particular, our work will be able to describe pair production in strong inhomogeneous fields in a more rigorous way than was so far feasible, improving eventually both the understanding of the positron production in strong quantum electrodynamic fields, as well as color string breaking in QCD, and the associated (strange) quark pair production. We therefore follow closely the experimental developments both regarding strange particle production in relatiyistic nuclear collisions as well as positron production in collisions of high Z-nuclei at the Coulomb barrier. 
Providence, Rhode Island 01912

Title of Project: Research and Development of a Helium-4-Based Solar Neutrino Detector

Person in Charge: $\quad$ R. E. Lanou

Telephone:

(401) 863-2632

Scientific Staff: $\quad$ H. Maris, G. Seidel, C. Enss

FY 1992 Funding: $\$ 150,000$

This project has as its goal the development of a technique to detect, count, and measure the energy of neutrinos created in the basic nuclear process between two protons that powers the Sun. The importance of carrying this out derives from the experimental observation that too few neutrinos have been seen from the solar nuclear processes. The two explanations of this effect, one of which is likely to be correct, are either that the neutrino has previously undetected properties or that our present model of how the Sun functions is incorrect or not sufficiently detailed.

Presently, the means do not exist for detecting and sampling in real time the energy deposited on Earth by these neutrinos from the proton-proton reaction with sensitivity to both charged and neutral current interactions. The project here is to utilize as a detecting medium the dominant helium isotope at very low temperatures; that is, in its superfluid state. Helium suggests itself because of its freedom from radioactive contamination and because the excitation energy deposited by the recoil from the scattered neutrino generates a high multiplicity of carriers, thus promising good resolution and low thresholds.

In experiments to date, we have seen the first detection of $5.5 \mathrm{MeV} \alpha$ particles via evaporation from a bath of superfluid helium. An $\alpha$ particle excites phonons and rotons in the liquid helium, and these excitations are sufficiently energetic to evaporate helium atoms when they reach the free surface of the liquid. The evaporated atoms are detected calorimetrically and in coincidence by thin wafers suspended above the liquid. The approximate overall efficiency of this process has been determined, and the experimental results compare favorably with expectations. We have also been able to detect evaporation by recoil electrons induced by a flux of gamma-rays from a Cs-137 source.

During the next continuation year, we shall work on improving the sensitivity of our prototype detector using superfluid helium. We shall investigate a number of materials to find the most suitable dielectric wafers for use in the millikelvin temperature range. We shall also convert our temperature sensing elements to superconducting transition edge bolometers. Experiments involving the use of multiple wafers and a movable source in the liquid will be used to study the quantum evaporation process and the reflectivity of rotons from liquid-solid interface. 
Title of Project: Investigation of Rare Particle Production in High Energy Nuclear Collisions

Person in Charge: H. J. Crawford

Telephone: (510) 486-6962

Scientific Staff: J. M. Engelage

FY 1992 Funding: $\quad \$ 105,000$

High energy nuclear collisions produce an environment that is not possible to make with any other process on Earth, and new forms of matter not observed previously may emerge from this environment. Collisions between beams of silicon and gold nuclei from the BNL AGS with nuclear targets lead to enhanced production of strange particles, and this enhanced strangeness may condense into new types of particles such as multiply strange nuclei or even lumps of strange matter such as strangelets. Our experimental program is an investigation of the production of antinuclei and a search for new types of matter formed in these collisions. Our goal is to understand how massive particles like antiprotons and antideuterons are produced from the conversion of kinetic energy of the beam into mass and to see whether any new types of particles emerge from these interactions.

Our current research program involves using a simple beam-line magnetic spectrometer to examine the particles produced in collisions of $\mathrm{Si}$ and $\mathrm{Au}$ beams with $\mathrm{Al}, \mathrm{Cu}$, and $\mathrm{Au}$ targets. The particles emerging from the interactions into a small solid-angle centered at 0 degrees are magnetically guided away from the interaction and passed through a series of plastic scintillation detectors, gas drift chambers, and Cerenkov radiators to determine their charge, mass, and momentum.

In FY 1992, we took data with the first high energy Au beam and have measured particle spectra over a broad range in momentum and mass. We are currently analyzing these data, looking for antinuclei and for any particles having a charge/mass ratio indicative of a new form of matter. We expect to have another high intensity run in FY 1993. 
Department of Physics

Berkeley, California 94720

Title of Project: $\quad$ Medium Energy Meson Physics

Person in Charge: $\quad$ K. M. Crowe

Telephone: $\quad$ (510) $486-5021$

Scientific Staff: $\quad$ D. Armstrong

FY 1992 Funding: $\$ 222,000$

QCD is the modern theory of the strong interaction. This theory is reasonably well understood at high energies, but, unfortunately, low energy QCD is still not well understood as it is far from being adequately tested. The Crystal Barrel experiment is designed to provide some of the tests. The basic line of research involves meson spectroscopy, analyses bearing on the quark and/or gluon content of nuclear states, and the exploration of mechanisms and rules that govern ppbar annihilation dynamics. The Crystal Barrel Detector has been installed at the Low Energy Antiproton Ring at CERN. It detects and identifies charged and neutral particles with a detector solid-angle efficiency close to 100 percent. The principal component of the detector is an array of 1,380 CsI crystals. These crystals surround a Jet Drift Chamber (JDC) located in a 1.5 Tesla magnetic field which measures the tnomentum and $\mathrm{dE} / \mathrm{dx}$ of charged particles. One of the very interesting physics goals of the detector is a search for exotic mesonic states-glueballs and hybrids. Annihilation at rest will be studied with a liquid hydrogen target and a gaseous target. The gaseous target offers the possibility of triggering atomic $L x$ rays so that specific initial angular momentum states can be studied. Our group was responsible for the construction and operation of JDC. The e reriment began in 1989 and will continue for several years.

Muon-catalyzed fusion of hydrogen isotopes was discovered in 1956 by Alvarez's group in the Berkeley bubble chamber. Our muon-catalyzed fusion program has been carried on at the Paul Scherrer Institute in Zurich. The goal is to investigate the basic physics processes involved in this reaction and which limit the ultimate number of fusions one muon can catalyze. We have recently completed the first direct measurement of "final sticking in dT fusion" (where the muon remains bound to the alpha product after it comes to rest). Our results show the first clean picture of this process and indicate a substantial deviation from theoretical expectations. From this experiment, we have also observed unexpectedly fast molecular formation, which we will continue to explore in a new high temperature target capable of reaching $2000 \mathrm{~K}$ at pressures up to 2,000 bars. 


\section{UNIVERSITY OF CALIFORNIA, LOS ANGELES}

Department of Physics

Los Angeles, California 90024

Title of Project: Intermediate Energy Nuclear Physics

Persons in Charge: G. Igo, C. A. Whitten

Tolephone: (310) 825-1306

Scientific Staff: $\quad$ S. Trentalange, V. Ghazikhanian

FY 1992 Funding: $\$ 354,000$

The major goal of the UCLA Intermediate Energy program is to determine the spin structure of the nucleon (neutron and proton) due to the three valence quarks known to exist in the nucleon (and due also to the "sea" quarks that appear spontaneously in the nucleon). An earlier deep inelastic experiment, European Muon Collaboration (EMC), on the proton only suggests that the quarks' spin angular momenta cancel one another on average and that the observed spin angular momentum of the nucleon must be due to other sources. This has been dubbed the "spin crisis" because it was so totally unexpected. The Spin Muon Collaboration, of which the UCLA Intermediate Energy Group is a member, began a program to measure the spin structure functions $g_{l}^{p}(\mathrm{x}) g_{l}^{n}(\mathrm{x})$ for the neutron and proton in 1991. That measurement is continuing in 1992 and projected for 1993 and 1994 and will provide an improved data sample with reduced statistical and systematic errors for $g_{f}(\mathrm{x})$. The experiment, sited at CERN, uses existing polarized $100-200-\mathrm{GeV}$ muon beams and an updated version of the EMC apparatus. Some of the detectors (multiple-wire proportional chambers) and some aspects of the polarized target are UCLA responsibilities.

Another component of the UCLA Intermediate Energy Program is the measurement, with high statistical precision, of some of the spin observables relevant to proton-deuteron elastic scattering. The data may be used to test the strong interaction between nucleons when a double-scattering process occurs between incident proton and the two nucleons of the deuteron, which proceeds with a spin flip of each of the nucleons. The interaction can be so brief that, according to the uncertainty principle, energy or momentum conservation may not be established (so-called "off-shell" interactions). Theoretical models of the nucleon-nucleon interaction, which provide good fits of on-shell data, predict different off-shell behaviors and may be tested by the three-body problem (in our case, proton-deuteron elastic scattering).

During 1992, a test run of one of the scintillating fiber hodoscopes and associated electronics at LNS Saclay was made. Two other scintillating fiber hodoscopes using multianode photomultipliers and additional electronics are currently under construction and testing. A data-taking run at LNS is projected (summer 1993), utilizing a complete set of scintillating fiber detectors and existing wire chambers. 


\section{UNIVERSITY OF CALIFORNIA, LOS ANGELES}

Department of Physics

Los Angeles, California 90024

Title of Project: Relativistic Heavy-Ion Physics

Persons ir. Charge: G. Igo, C. A. Whitten

Telephone:

(310) $825-1306$

Scientific Staff: J. Carroll, V. Ghazikhanian, T. Hallman, S. Trentalange

FY 1992 Funding: $\quad \$ 292,000$

At the LBL Bevalac, $e^{+} e$ production is measured in nuclear reactions. The most elementary interaction, $\left(\mathrm{e}^{+} \mathrm{e}\right)$.. production occurring in nucleon-nucleon $(\mathrm{n}-\mathrm{n})$ collisions, has been measured in proton-proton and deuteron-proton collisions at bombarding energies ranging from one to several $\mathrm{GeV} /$ nucleon. $\mathrm{e}^{+} \mathrm{e}$ production comes about due to bremsstrahlung and pion production and possibly other processes occurring in the $n-n$ collision. Differences in the observed e production in collisions of relativistic heavy ions with nuclei $\left(e^{+} e^{e}\right)_{\mathrm{RH}}$ and in the production predicted from $\left(\mathrm{e}^{+} \mathrm{e}\right)_{\mathrm{a} \text {. }}$ collisions between the nucleon constituents come about because of collective effects among the constituents of the colliding system. The e'e production mechanism is advantageous to study collective effects because $e^{\prime} e$ pairs emitted by the hot nuclear matter produced in a collision are relatively unscathed as they pass out of the nuclear medium. During the summer of 1992, final data on the (ete).. was obtained at the lower end of the bombarding energy range. In September 1992, the final data-taking for $\left(\mathrm{e}^{+} \mathrm{e}\right)_{\mathrm{RH}}$ production at bombarding energies up to about $2 \mathrm{GeV} /$ nucleon will take place.

A considerable fraction of our effort goes for research and development and administrative activities for the Solenoidal Tracker at RHIC (STAR) detector, projected as a major facility in the RHIC experimental program. R\&D efforts are focused on the design of an electromagnetic calorimeter for the STAR detector. We plan major programs using this dete:tor with emphasis on jet and $\gamma$ production in relativistic heavy-ion and polarized proton-protor, collisions. 
Title of Project: Particle Physics

Person in Charge: $\quad$ B. M. K. Nefkens

Telephone: $\quad(310) 825-4970$

Scientific Staft: M. Clajus, R. Kessler, W. Tippens, M. Wang

FY 1992 Funding: $\$ 374,(1)()$

The research carried out hy the UCLA Particle Physics Research Group is grouped into four programs.

Program I concerns the pion-nucleon interactions and meson photoproduction. The objectives include the determination of pi- $\mathrm{N}$ resonance characteristics: mass, width, and decay hranching ratios. A complete set of pi- $\mathrm{N}$ scattering amplitudes is being measured. Currently, we are concentrating our efforts on gamma $p->$ eta $p$ and gamma $d->$ eta $d$ near threshold. This work is carried out at ELSA (Bonn). Further research in eta photoproduction is being prepared for CEBAF; we also want to measure eta electroproduction near threshold there. At Gatchina (St. Petershurg), we are measuring pi $\mathrm{p} \rightarrow->$ eta $\mathrm{n}$, also near threshold.

Program II is concerned with tests of charge symmetry and isospin invariance in systems of a pion in combination with one, $(w 0$, or three nucleons. A new program is heing launched at AGS to test charge symmetry in eta-nuclear systems. Specifically, we are comparing d sigma(pi-d --> nn eta) with d sigma(pi+d --> pp eta).

The ${ }^{3} \mathrm{H}-{ }^{3} \mathrm{He}$ isospin doublet forms an attractive pair of nuclei for sensitive tests of charge symmetry. Experiments are carried out at LAMPF, where the capability exists for handling large quantities of tritium $\left({ }^{3} \mathrm{H}\right)$. Charge symmetry is investigated using pion elastic scattering on ${ }^{3} \mathrm{H}$ and ${ }^{3} \mathrm{He}$ using the novel techniques of the superratio.

Program III is focused on testing the Standard Model and making a search for New Physics using rare and forhidden decays of the eta meson and the muon. We have just completed a new, clean measurement of the branching ratio for the rare decay eta $->$ mu+ mu- using the eta factory at Saclay. In the process, we have obtained new values for the upper limits on eta --> pi+ pi- (forbidden hy CP), eta --> mu+- e-+ (forbidden hy lepton family conservation), and eta --> e+ e- (possible indirect evidence for the existence of leptoquarks).

An important new effort is the bringing into being of an eta factory at AGS using the nuclear production reaction pi $+\mathrm{d}-\rightarrow$ pp eta. This allows major tests of CP invariance outside the Standard Model, such as the search for the lepton charge asymmetry in the rare decays eta --> pio mu+ mu- and eta --> pio e+ e-. The detector is ETAS, a novel superconducting split solenoid spectrometer that has 70 percent solid angle acceptance. 
A large and sophisticated experiment is MEGA, a search for Mu decays to Electrons + GAmma, to test lepton family number conservation; it is done by a large collahoration at LAMPF.

Program IV covers theoretical analysis and technical developments in support of the previous programs. 
Department of Physics

Riverside, California 92521

Title of Project: Central Collisions of Heavy Ions

Person in Charge: $\quad$ S.-Y. Fung

Telephone:

(714) $787-5636$

Scientific Staff: $\quad$ S.-Y. Chu, J. Chang, J. H. Kang, R. Seto

FY 1992 Funding: $\$ 274,000$

The aim of this research is to learn about the interaction between nuclei at very high densities and ultrahigh energies. How nuclei interact under such extreme conditions is inherently and fundamentally interesting. The collisions of nuclei at 9 hundred billion electron volts are completely unexplored territories. This study will shed light on the most basic question in cosmology: the formation of the universe and the transition from the quark-gluon plasma to urdinary nuclear matter. In the first few microseconds after the "Big Bang," it was postulated that the universe must have been very dense and hot. Such an environment can be recreated and studied in the laboratory only through the central collisions of heavy ions at ultrahigh energy.

Research of the Riverside group focuses on particle correlation and global parameters describing central collisions of nuclei at relativistic energies. Experiments are conducted at the LBL Bevalac and at the BNL AGS. At LBL, we carried out a systematic study of the source size of the pion-emitting interaction region and observed a momentum dependence, consistent with the picture of an expanding fireball. We have just completed a proton-proton correlation study in lanthanum on lanthanum collisions using the Heavy-Ion Superconducting Spectrometer (HISS) system. For this large composite system, the source size could not be interpreted with existing theoretical models. We also used the global transverse momentum of charged secondaries to infer how nuclear matter is compressed in collisions. We found a medium to stiff nuclear compressibility associated with the nuclear equation of state at the Bevalac energies.

At the Brookhaven laboratory, we studied the fluctuations in pseudorapidity distributions of charged particles using scaled factorial moments. A power law growth of moments is observed down to a bin size of 0.1 . In the past year, the E859 Collaboration also reported findings on forward and transverse energies and antiproton production in heavy-ion collisions as well as particle production in protoninduced reactions at $14.6 \mathrm{GeV} / \mathrm{c}$. We have also begun the study of gold-on-gold collisions.

We are part of the PHENIX Collaboration at RHIC to be commissioned at Brookhaven in 1997. We will study the production of leptons, direct photons, and also of hadrons as potential quark-gluon-plasma signatures. 
Title of Project: $\quad$ Neutrino Physics

Person in Charge: $\quad$ G. J. Van Dalen

Telephone: (714) $787-3414$

FY 1992 Funding: $\quad \$ 100,000$

Neutrino interactions have been used throughout the development of particle physics to study a wide range of fundamental questions including the nature of the electroweak interaction, lepton number conservation, and as a probe of cosmology. Low energy neutrinos, such as those available at LAMPF, offer significant advantages in testing the fundamental properties of the neutrinos themselves. In the 1980s, a series of experiments at LAMPF measured neutrino interactions at low energies. Since 1982, several members of our group have participated in experiment E764 at LAMPF, which established significant limits in the search for neutrino oscillations, and reported measurements of muon neutrinoinduced nuclear transition cross sections.

We are now collaborating in a new program, which is a natural successor to the previous experiment and serves as a logical next step in the entire LAMPF neutrino program. The primary goal of the experiment is to search for neutrino oscillatious to the levels of $0.02 \mathrm{eV}^{2}$ in mass difference and $2 \times 10^{-4}$ in mixing, greatly extending the range explored by accelerator-based searches. The new experiment, called the Large Scintillation Neutrino Detector (LSND), will consist of 200 tons of dilute mineral oilliquid scintillator located near the LAMPF beam stop. The primary focus of LSND is neutrino oscillations, although a range of related neutrino interactions will be investigated.

The first stages of construction are underway, and data acquisition with the complete detector will hegin in early 1993. The Riverside group will focus early work on the detector readout, data acquisition, and online processing. We bring experience from OPAL at Large Electron Positron with related systems. We view LSND as both a logical step from earlier neutrino experiments at LAMPF and as a precursor to more ambitious plans that will include testing the electroweak Standard Model at low momentum transfer in neutrino scattering with the proposed Large Cherenkov Detector. 
Physics Department

Pasadena, California 91125

Title of Project: $\quad$ Properties on Nuclei and Elementary Particles

Person in Charge: F. Boehm

Telephone: $\quad$ (818) $356-4266$

Scientific Staff: $\quad$ H. Henrikson, N. Mascarenhas, V. Novikov, E. Ormand, P. Vogel, A. Young

FY 1992 Funding: $\quad \$ 738,000$

Research in the Norman Bridge Laboratory at Caltech is aimed at the fundamental properties of elementary particles as manifested in nuclear processes.

During the past several years, our research has focused on neutrino mass and mixing of different neutrino states. Previous experiments by this group, using a nuclear power reactor as a neutrino source, have shown convincingly that neutrino mixing as proposed in some theoretical models, if it exists at all, must he very small, as must be the masses of the neutrinos.

Incorporating new scintillation counter technology, we have now designed a 12-ton neutrino detector to he installed at a distance of about $1 \mathrm{~km}$ from the San Onofre power reactors. With such a large low background detector, we shall enhance the mass sensitivity in these experiments to include a mass region suggested by current solar neutrino experiments.

In related investigations, results were obtained from our double-beta-decay experiment in the Gotthard Tunnel ( $1,500 \mathrm{~m}$ helow the surface), using a TPC. Our data from this experiment, which employs $1,5(0)$ liters of enriched ${ }^{136} \mathrm{Xe}$ at $5 \mathrm{~atm}$, now tell us that neutrino mass must be less than $1 \mathrm{eV}$, a finding that has strong bearing on cosmological models.

The possibility of the admixture of heavy neutrinos to the usual light neutrino has been suggested. A sensitive study of a beta spectrum from ${ }^{35} \mathrm{~S}$ near its endpoint has been undertaken with the use of our Caltech double-focusing beta spectrometer. No evidence for a heavy neutrino, such as the one proposed with mass of $17 \mathrm{keV}$, has been seen. As a consequence, neutrino mixing strengths of larger than 0.2 percent can be ruled out.

In pursuit of the intriguing and still unresolved question whether time reversal symmetry holds exactly in nuclear systems, we are conducting a new set of measurements of the spatial symmetry of radiation emitted from polarized nuclei, employing our ${ }^{3} \mathrm{He}-{ }^{4} \mathrm{He}$ dilution refrigerator.

On the theoretical side, we have calculated the rates for double-heta decay into two electrons and two antineutrinos and also into two electrons in various nuclei using the Quasiparticle random phase approximation (QRPA) technique. This allows us to interpret our experimental results in terms of a value or limit of the neutrino mass. Calculations have also heen performed on interactions of hypothetical Dark Matter particles with nuclei as well as on CP and $\mathrm{T}$ violations in nuclear systems and on "seniority truncation" in the nuclear shell model. 


\section{CALIFORNIA STATE UNIVERSITY, NORTHRIDGE}

Department of Physics and Astronomy

Northridge, California 91330

Title of Project: Theoretical Research in Intermediate Energy Nuclear Physics

Person in Chargis: $\quad$ R. Seki

Telephone: (818) $885-2773$

FY 1992 Funding: $\quad \$ 82,000$

A recent, fundamental challenge in nuclear physics is understanding nuclear structure and reactions in terms of QCD, which is believed to be the microscopic basic theory of the hadrons. Traditionally, a nucleus is described in terms of nucleons and the mesons that mediate their interactions. QCD could provide unified and deeper understanding of the nuclear phenomena in terms of more fundamental constituents, quarks and gluons. With this aim, this research has two objectives: to understand the nucleon-nucleon attraction in intermediate distances and to access nuclear effects in the single-proton knockout from nuclei in high energy electron scattering.

Because QCD is not yet manageable as a description of low energy nuclear phenomena, we use the Skyrme model to achieve the first objective. The model is not QCD but embodies its essential consequences and is amenable to direct computations, although they are still complicated. In this model, the nucleon is described as a solitary wave (soliton) of classical pion fields and the nucleon interactions as distortions of the solitons as they come close to each other. The description differs from the traditional meson-exchange description, in which the nucleon is considered basically pointlike without dynamical structure. The studies are carried out by a combination of algebraic and numerical calculations, both of which are carried out by the use of computers because of complexity.

QCD is believed to be manageable in high energy phenomena. It is expected to yield an unexpected consequence that a knocked-out high energy proton from a nucleus is much smaller than the ordinary proton and experiences little nuclear interaction as it goes out from the nucleus. This strange phenomenon is called color transparency, and an experiment has been performed at the SLAC to observe it by the use of higl: energy electron beams. Calculations are being made to evaluate various nuclear effects in this reaction so as to isolate the true consequence of this strange phenomenon.

Future work will focus directly on QCD itself, such as correlation functions of meson currents in the QCD vacuum. The correlation functions reveal the nature of the vacuum in which complicated hadronic phenomena take place. 
Department of Physics

Pittsburgh, Pennsylvania 15213

Title of Project: Experimental Medium Energy Physics

Person in Charge: $\quad$ G. B. Franklin

Telephone:

(412) $268-2743$

Scientific Staff: $\quad$ B. Quinn, R. A. Schumacher, R. Magahiz, V. Zeps

FY 1992 Funding: $\quad \$ 767,000$

The Experimental Medium Energy Physics Group at Carnegie Mellon designs and executes experiments that probe our understanding of what are believed to be the fundamental laws of physics. Although many of these laws have been shown to work well at very high energies, theoretical models developed to utilize them to explain the existence of ordinary subatomic particles (such as protons and neutrons) and their interactions are incomplete. In particular, we wish to understand these particles in terms of the interactions of their constituents, quarks.

Although ordinary nuclear matter is thought to be constructed mostly of two types of quarks, "u-quarks" and "d-quarks," we have performed several experiments that involve the creation of a third type, the "s-quark." These experiments have provided insight into the interaction of quarks at energies that exist within ordinary matter.

We are currently conducting experiments to search for a six-quark object called the H-Dibaryon and long-lived nuclei with multiple strange quarks called "strangelets," both at BNL. The development of the equipment for the H-Dibaryon search took several years and is now performing as planned. We expect our first results from this measurement late in 1992. We have also just completed the analysis of an experiment that measured the weak interaction between a lambda and a nucleon.

We are now developing the apparatus to perform additional s-quark production studies at CEBAF. We also have plans to search for the presence of s-quarks and anti-s-quarks in the proton in another CEBAF experiment. 
Department of Chemistry

Pittsburgh, Pennsylvania 15213

Title of Project: Nuclear Research with Heavy Ion

Person in Charge: M. Kaplan

Telephone:

(412) 268-3123

Scientific Staff: $\quad$ Z. Milosevich, E. Vardaci

FY 1992 Funding: $\quad \$ 190,000$

One of the most important areas of nuclear science is concerned with the behavior of nuclear matter under conditions of very high temperatures, pressures, and rotational forces. Our experimental research program addresses this area by focusing on transient excited states of atomic nuclei which are produced in violent collisions between energetic heavy-ion projectiles and complex target nuclei. We study the reaction mechanisms, de-excitation pathways, relaxation modes, and space-time correlations of very hot (highly excited) nuclei as functions of controlled excitation energy, composition, and angular momentum. In support of the experimental efforts, we maintain developmental activity in several theoretical and computational projects that play an important role in the analysis and interpretation of the experimental data and are instrumental in the design of new experimental approaches.

The current research program is directed toward developing a thorough understanding of the role of the compound (or composite) nucleus and its decay properties in medium energy nuclear reactions. We pose the following questions: (1) What is the maximum energy deposition that a nucleus can absorb, and how does it depend on mass? (2) Is there a maximum temperature at which a nucleus can exist as an equilibrium system? (3) Is there a maximum spin that a nucleus can accommodate, and how does spin affect the shape? (4) Does the shape of the highly excited composite nuclei exhibit fluctuations or tails in the matter density distribution? If such tails exist, are they entrance channel effects? Relaxation effects? Closely related to such concepts are the effective levei densities of hot and/or distorted nuclei and their influence on the competition between decay modes.

In parallel with our experimental objectives of addressing the previous questions, our program has undertaken a major new initiative by joining the Solenoidal Tracker at RHIC (STAR) Collaboration, a consortium of research institutions dedicated to the design, construction, and implementation of the STAR Detector System for RHIC. Our immediate responsibilities in this initiative involve detailed computer simulations for the multilevel triggering capabilities of the STAR detector. 


\section{UNIVERSITY OF CHICAGO}

Department of Astronomy and Astrophysics

Chicago, Illinois 60637

Title of Project: $\quad$ Nuclear Physics and Astrophysics

Person in Charge: $\quad$ D.N. Schramm

Telephone:

(312) $702-8206$

$\begin{array}{ll}\text { Scientific Staff: } & \text { A. Olinto, D. Thomas } \\ \text { FY } 1992 \text { Funding: } & \$ 76,000\end{array}$

The interface between nuclear physics and astrophysics has become a fundamental and exciting area of research in recent years. The study of matter at the extremely high temperatures and densities found in the very early universe and in stellar objects serves as an input to our theories of nuclear interactions and also profits from them. For example, the properties of neutrinos are strongly constrained by the comparison of astronomical observations of light element abundances with the predictions of Big Bang nucleosynthesis, which describes how elements formed when the universe was a fraction of a second old. Similarly, our understanding of supernovae mechanisms and of neutron stars strongly constrains and is in turn constrained by the properties of nuclear matter and neutrinos. The goal of this research program is to further develop this interdisciplinary area and enhance our understanding of both nuclear physics and astrophysics.

Within this broad interface area, our research has focused on a variety of topics. One of the efforts has been the study of nuclear matter at the extreme densities found in neutron stars. We have studied the constraints imposed on nuclear models by laboratory experiments and neutron star observations. This includes study of the implications of a possible stable state of quark matter and of metastable dihyperon states. A second objective has been the study of consequences for the element abundances of inhomogeneous Big Bang nucleosynthesis and the possibility of synthesizing higher mass elements, for example, boron and berillium, in the early universe. Another area of exploration has been the impact of astrophysical uncertainties on the solar neutrino problem.

One of the recent developments in this program has been the realization that major changes in the structure of neutron stars may arise from the presence of dihyperon states in dense matter. The investigation of boron and berillium abundances has shown that these elements can be primordially produced in greater amounts than previously thought but still below the observations. The study of the solar neutrino problem has shown that the allowed parameter space for the MSW solution is broader than previously understood.

Upcoming experimental and observational results from Brookhaven on dihyperons and strangelets; from RHIC on the quark-gluon plasma; from Soviet-American Gallium Experiment, GALLEX, and Sudbury Neutrino Observatory on the solar neutrino flux; from element abundances in different astronomical objects; from ROSAT and AXAF on neutron star observations; and from many other sources will facilitate a better understanding of all the different aspects of this project. Future plans 
include the study of laboratory results and their implications for dense matter, improvement in Big Bang nucleosynthesis constraints on higher mass elements, constraints on first-order phase transitions in the early universe due to nucleosynthesis bounds, and study of neutrino interactions in the Sun and in supernova 1987a. 
Department of Chemistry

Worcester, Massachusetts 01610

Title of Project: $\quad$ Nuclear Structure Research

Person in Charge: $\quad$ D. S. Brenner

Telephone: $\quad$ (508) 793-7114

Scientific Staff: $\quad$ W.-T. Chou, N. V. Zamfir, A. Wolf

FY 1992 Funding: $\quad \$ 145,000$

Our research program in nuclear structure is a collaborative effort involving Clark University personnel, staff members from BNL, and an active participation of scientists from other nations. The TRISTAN on-line isotope separator and the tailored beam facility at the High Flux Beam Reactor (BNL) are the experimental foci of our research program, which is aimed at understanding the critical factors that give rise to nuclear stability. We are working to develop a framework for understanding the structures and properties of radioactive nuclei that can be studied in the laboratory, so that this framework or scheme can be used to predict the properties of nuclei that we cannot now produce and study here on Earth. Such nuclei, which undoubtedly are created in stars and supernovae, are keys to understanding the creation of matter in the universe.

Recent research has been two-pronged. Experimental studies of nuclei with unusual structural features have been pursued both at BNL and at the Institute Laue-Langevin, France. In tandem with these efforts have been several initiatives to characterize nuclei and their properties such as the mass, shape, and moment of inertia, in terms of the number valence protons and neutrons and the interactions among these. We have had remarkable success in the latter endeavor by using a simple counting scheme that compares the number of like-particle interactions to the number of proton-neutron interactions.

We expect to continue our work along current lines and expand our collaborations with scientists from abroad. Future work will seek to exploit our understanding of relatively stable nuclei in order to predict the properties of unknown, and presumably much less stable, ones. Ultimately, we hope to use a new experimental facility, the Isospin Laboratory, currently in the initial planning stage, to produce and study the properties of these exotic nuclei. 


\section{UNIVERSITY OF COLORADO}

Department of Physics

Boulder, Colorado 80309

Titlo of Project: Intermediate Energy Nuclear Physics

Person in Charge: R. J. Peterson

Tolephone: (303) $492-7483$

Sciontific Staff: $\quad$ R. A. Ristinen, E. R. Kinney

FY 1992 Funding: $\$ \$ 541,000$

We use beams of nucleons, electrons, and mesons to investigate nuclear reaction mechanisms and nuclear structure. We begin with fundamental measurements of pi meson cross sections and analyzing powers on nucleons and deuterons to establish the foundations.

Recent studies on complex nuclei compare the response of the target at high momentum transfer to a range of scattering probes. We use polarized proton beams at LAMPF for isovector spinflip responses and both $\pi$ mesons and $\mathrm{H}^{+}$mesons to study isoscalar and isovector nonspin responses. At moderate momentum transfers, we investigate nuclear spin excitations by pion scattering and nucleon transfer reactio.s. We find a severe shortage of isoscalar spinflip strength to simple stretched states, relative to general sum rules. Although the isovector strength may be understood in terms of occupation numbers in the valence shells, the isoscalar strength shortage must come from more fundamental causes.

Our future plans are to use the new Neutral Meson Spectrometer at LAMPF for more detailed studies of the isovector response of nuclei. Using the good resolution of this instrument will allow us to use known nuclear quantum numbers for discrete states to specify the terms in the scattering. 
Title of Project: Theoretical Nuclear Physics

Persons in Charge: E. Rost, J. R. Shepard

Telephone: $\quad$ (303) 492-3695

Scientific Staff: $\quad$ P. D. Kunz, C. E. Price

FY 1992 Funding: $\$ \$ 172,000$

The most fundamental theories in physics today are relativistic quantum field theories (RQFTs). The archetypal theory of this kind is quantum electrodynamics (QED) which, as the name implies, addresses the physics of electromagnetic interactions such as those which determine the structure of atoms. QCD is generally accepted as RQFT for strong interactions which, for example, are responsible for the properties of nuclei. Unfortunately, QCD is-at present-a theory "everyone can write down but no one can solve." Although this statement is overly pessimistic, it is true that successful description by QCD of many basic nuclear phenomena must await conceptual and calculational breakthroughs. Hence, simpler RQFTs which incorporate some of the important properties of QCD but are tractible when applied to nuclear physics problems, are of great current interest. Study of such models is the major focus of our research program.

A distinctive characteristic of RQFTs is the presence of an infinite "sea" of negative energy fermions in addition to their positive energy counterparts which are the only oncs present in nonrelativistic theories. Because the sea can interact dynamically with the positive energy fermions, it can affect properties of many-fermion systems such as the nuclei that appear in an effective RQFT for nuclear structure known as quantum hadrodynamics (QHD). Much of our current research has focused on treating sea effects in theories such as QHD. Because of unique conceptual and mathematical difficulties associated with sea contributions, they are typically treated (if at all!) by making the simplifying assumption that the fermion system is uniform and, hence, infinite in extent.

In recent work, we have formulated a method of treating certain sea contributions exactly for finite systems. This is crucial since not only are the only known nuclear systems finite, they are strongly affected by, e.g., surface effects which are absent in infinite systems. We have demonstrated in model calculations of nucleons (in a chiral soliton model) as well as nuclei in one spatial dimension that our method works, i.e., reproduces previous calculations, and that it is extremely efficient.

Future work will concentrate on the extension of our method to treat sea contributions in three spatial dimensions. This is a big step calculationally, but we have already been successful in treating 1-loop sea contributions for a self-interacting scalar field and have also outlined a three-dimensional approach for fermions. 
Department of Physics

Golden, Colorado 80401

Title of Project: $\quad$ Nuclear Reactions at Very Low Energies

Person in Charge: $\quad$ F. E. Cecil

Scientific Staff: $\quad$ J. A. McNeil, C. S. Galovich, H. Liu

FY 1992 Funding: $\quad \$ 118,000$

The objective of our project is the measurement and application of nuclear reaction cross sections (probabilities) between ordinary light ions (protons, deuterons, alpha particles) and low- $Z$ targets (through boron). The reactions are investigated at low energies (below about $200 \mathrm{keV}$ ). These low energies are appropriate for the application of our measurements to a variety of applications in the fields of stellar and primordial Big Bang nucleosynthesis and high temperature fusion plasma diagnostics. The measurements are carried out with the low energy, high current charged particle accelerator at the Colorado School of Mines Applied Nuclear Physics Laboratory. The machine operates at energies from 0 to $180 \mathrm{keV}$ and with beam currents from nanoAmperes to about 1 milliAmpere.

Recent specific measurements include the radiative capture of protons by ${ }^{6} \mathrm{Li},{ }^{7} \mathrm{Li},{ }^{9} \mathrm{Be},{ }^{11} \mathrm{~B}$ at energies between about 25 and $180 \mathrm{keV}$. The specific application of these reactions is the diagnostics of the proposed "aneutronic" advanced fuel cycle fusion devices. In parallel with these measurements, a series of direct reaction calculations was carried out and found to be in excellent agreement with the measured cross sections.

Another series of recent measurements were devoted to the resolution of a classic problem in nuclear physics, "the Oppenheimer-Phillips (OP) effect," in which nuclear reactions involving deuterons may be altered as a result of the distortion of the deuteron in the electric field of the target. We measured single nucleon transfer reactions between deuterons and ${ }^{2} \mathrm{H},{ }^{6} \mathrm{Li}$, and ${ }^{10} \mathrm{~B}$ down to energies of 3,25 , and $50 \mathrm{keV}$, respectively, and found no evidence of the enhancement of the reactions expected on the basis of the OP effect.

Measurements in progress include the $\mathrm{d}-\alpha$ and ${ }^{3} \mathrm{He}-{ }^{7} \mathrm{Li}$ reactions, which are of particular relevance to the field of primordial nucleosynthesis. 
Department of Physics

Nevis Labs

Irvington, New York 10533

Title of Project: Relativistic Heavy-Ion Research

Person in Charge: S. Nagamiya

Telephone:

(212) 854-3334

Scientific Staff: $\quad$ B. Cole, C. Y. Chi, T. Nayak, W. Zajc

FY 1992 Funding: $\quad \$ 760,000$

The goal of this research is to explore experimentally new and unique features of relativistic heavy-ion collisions. Because the recent availability of high energy beams of nuclei at BNL and CERN provides unprecedented opportunities to create dense, highly excited nuclear systems, special emphasis is given to the search for new phases associated with dense nuclear matter, in concert with careful measurements of baseline phenomena.

The group has participated in the development of a large scale experiment (E802) and its successor (E859) at BNL. These experiments, consisting of approximately 70 physicists, have made precision measurements of single- and two-particle inclusive distributions. The Columbia group has participated at many levels. We have built a high pressure segmented Cerenkov counter essential for particle identification at high momenta; we took major responsibility for the development of the Monte Carlo for the experiment, shared in the design and implementation of the trigger for E859, and contributed to the management of the experiment (S. Nagamiya was cospokesman for E802; W. Zajc for E859).

Two notable results from this program are the observations and the first measurement of a two-kaon correlation function.

The Columbia group will continue its involvement in the successor to E859 (E866), which involves a significant upgrade of the experiment to handle the much higher multiplicities expected from $\mathrm{Au}$ beams. In addition, a major effort has begun to design an experiment for RHIC at BNL. The proposed experiment, called PHENIX, will be an international collaboration of more than 280 physicists, with S. Nagamiya as spokesman. 
Title of Project: $\quad$ A Pion Interferometry Search for a Phase Change in Hadronic Matter in Relativistic Heavy-Ion Collisons and Its Application to RHIC

Person in Charge:

Telephone:

Scientific Staff:

FY 1992 Funding:
M. Cherney

(402) 280-3039

J. Seger, T. McShane

This project studies the behavior of nuclear matter under extreme conditions. At high temperatures and high density, it is predicted that nuclear matter will undergo a phase change. At sufficiently high temperatures and densities, quarks will cease to belong to a single nucleon and a quark-gluon plasma will be formed. The objective of this project is to search for signatures of a quark-gluon plasma phase of matter.

The research program consists of the analysis of data from the NA36 experiment taken at CERN, the collection of data with the NA44 experiment at CERN, and design of studies for the Solenoidal Tracker at RHIC (STAR) experiment at BNL. The project stresses active participation of Creighton undergraduate and Master's degree siudents.

Studies of the NA36 detector resolution were undertaken in order to determine the feasibility of using pion interferometry as a tool in the search for signatures of a quark-gluon plasma in the NA36 experiment in sulfur-nucleus collisions. Data are currently being collected at the NA44 experiment. This experiment is a spectrometer designed for studying correlations in the production of pairs of identical particles with similar momenta. In addition to active involvement in data collection from proton-nucleus and sulfur-nucleus interactions at the CERN Super Proton Synchrotron, the group is involved in the construction of a spot focusing Cerenkov detector and an upgrade to the experiment's data acquisition system for the study of lead-nucleus interactions.

Computer studies of tracking of charged particles, produced in gold-gold collider interactions through a central TPC, are being carried out for the STAR experiment. Slow control systems and the related data acquisition system are also being evaluated for STAR. 
Granville, Ohio 43023

Title of Project: $\quad$ Neutron Cross Section Measurements Using ORELA

Person in Charge: $\quad$ R. R. Winters

Telephone: (614) $587-6227$

FY 1992 Funding: $\quad \$ 48,000$

The program has three primary goals: (1) to provide neutron capture cross sections over the energy range of 2 to $200 \mathrm{keV}$, important in stellar nucleosynthesis of the chemical elements heavier than iron; (2) to study the hehavior of the optical model potential (OMP) just above neutron binding; and (3) to provide neutron total cross sections and R-matrix descriptions for use in nuclear reactors. All cross section measurements are made using the Oak Ridge Electron Linear Accelerator (ORELA) in collaboration with staff scientists at ORNL. The work is now concentrating on the hehavior of OMP near the nuclear closed neutron shells. Measurements of the neutron total cross sections for $n+{ }^{8 x} \mathrm{Sr}$, $\mathrm{n}+{ }^{91} \mathrm{Zr}$, and $\mathrm{n}+{ }^{28} \mathrm{Si}$ are planned during this funding period.

We recently measured the neutron scattering cross section for $n+{ }^{90} \mathrm{Zr}, \mathrm{n}+{ }^{88} \mathrm{Sr}$, and $\mathrm{n}+{ }^{28} \mathrm{Si}$. These measurements cover the neutron energy range of $\sim 0.015$ to $0.5 \mathrm{MeV}$ and will allow assignment of spin and parity of the resolved resonances. These data, coupled with the neutron total cross section data, will allow determination of an R-matrix description of the cross section for the partial waves, $\ell=(), 1,2$ for spins $\mathrm{J}=0.5,1.5,2.5$. The scattering functions can be calculated from the resulting neutron strength functions and external $\mathrm{R}$-functions for each partial wave. These scattering functions are then described by searching on the real and imaginary well depths of OMP for each partial wave. The contribution of the ORELA data is to provide estimates of the contribution, via the dispersion relation, of the imaginary well depths to the central real potential. The resulting potentials can then be used to calculate the positions of the bound states in the compound nucleus, which can be compared with measured values. Thus, the idea that OMP should smoothly join the shell model potential can be tested. 


\section{TRIANGLE UNIVERSITIES NUCLEAR LABORATORY AT DUKE}

Durham, North Carolina 27706

Jointly staffed by Duke University, North Carolina State University, and the University of North Carolina. See each of these universities for scientific staff and research programs. The Triangle Universities Nuclear Laboratory (TUNL) is located at Duke University.

Title of Project: $\quad$ TUNL Accelerator Facility

Person in Charge: N. R. Roberson

Telephone: (919) 660-2600

FY 1992 Funding: $\quad \$ 830,000$

The major part of the TUNL experimental program utilizes an upgraded FN Tandem Van de Graaff accelerator that provides polarized and unpolarized beams up to $20 \mathrm{McV}$ for singly charged ions. The Atomic-Beam Polarized Ion Source installed in 1989 provides 10 microamperes of negative polarized protons and deuterons at the low energy end of the Tandem. A bunching system produces subnanosecond pulsed beams. A Low Energy Beam Facility (LEBF) makes use of the 100 microamperes of positive polarized heam for experiments in the 30-100-keV range. Part of LEBF is an "in-air" mini-tandem accelerator, biased by a high-voltage power supply, capable of accelerating the low energy negative polarized heams up to an energy of $350 \mathrm{keV}$. Additional ion sources include a direct-extraction negative-ion source for p! vions and deuterons and a charge-exchange source for $\mathrm{He}$ and $\mathrm{Li}$ ions. A single-ended Van de Graaff is used for high energy resolution studies of protoninduced resonances at energies between $500 \mathrm{keV}$ and $4.1 \mathrm{MeV}$.

Major experimental equipment at TUNL includes the following: (1) a 10-milliKelvin, 7-Tesla, cryogenic polarized target facility for transmission experiments with polarized neutrons; (2) a rotating 200-milliKelvin cryogenic polanized target for experimental test of time-reversal invariance; (3) four large Nal gamma-ray spectrometeıs and a recoil particle detector for radiative capture measurements; (4) a time-of-flight facility for polarized neutron scattering research with nanosecond heam pulses; (5) two 24-inch scattering chambers for angular distributions of charged particles; (6) an array of 11 neutron detectors for studying breakup reactions; (7) a computer system for data acquisition and analysis consisting of a network of 12 DEC VS3200s, 18 Macintosh computers, and 3 Sun workstations; and (8) a high resolution gamma-ray spectrometer consisting of two large high purity Germanium detectors with a BGO anti-Compton shie'.d.

Additional facilities under development include the following: (1) an Enge split-pole magnetic spectrometer being refurbished for research in few-body systems and nuclear astrophysics; (2) a new cryostat for bolometric particle detectors; and (3) permanent magnet sextupoles for the atomic heam polarized ion source to increase the atom beam fluxes. 
Physics Department

Durham, North Carolina 27706

Work Performed at the Triangle Universities Nuclear Laboratory (TUNL)

Title of Project: Studies of Nuclear Structure Using Neutrons and Light Ions

Person in Charge: N. R. Robe sson

Telephone: $\quad$ (919) 660-2600

Scientific Staff: $\quad$ E. G. Bilpuch, R. Chasteler, E. Crosson, C. R. Howell, L. H. Kramer, W. Tornow, B. Vlahovic, R. L. Walter, H. R. Weller, C. R. Westerfeldt

FY 1992 Funding: $\quad \$ 790,000$

Investigations of Fundamental Symmetries A major goal of nuclear physics is to study how the symmetries of space and time affect the forces that act between nucleons in nuclei. At Los Alamos, our work is with the TRIPLE collaboration, and we study parity violation in epithermal neutron resonances in heavy nuclei. At TUNL, we have carried out the first measurement of a bound on T-violation in polarized neutron transmission through a cryogenically aligned target. (For further details, see North Carolina State University-Mitchell).

Statistical Properties of Nuclear Energy Levels and Tests of Chaos in the Nucleus The ultrahigh beam energy resolution available with the single-ended TUNL accelerator provides unique data for the test of statistical theories and chaotic behavior in nuclei. (For further details, see North Carolina State University).

Radiative Capture Reactions Our studies of few-body nuclear reactions have proven that the $\mathrm{d}+\mathrm{T}$ and $\mathrm{d}+{ }^{3} \mathrm{He}$ fusion reactions are driven by the tensor force. We have also observed and measured the D-state in ${ }^{8} \mathrm{Be}$ for the first time. We are presently using the intense pqlarized deuteron beam directly out of the Atomic-Beam Polarized Ion Source to study these reactions at very low $(<80 \mathrm{keV})$ energies. One completed study has shown that previous theories of the $d+d$ fusion reaction are incorrect and has stimulated new theoretical work in this field.

Neutron Scattering Physics The comparison of two- and three-nucleon scattering data to calculations is providing new insight about the nucleon-nucleon (NN) force and few-nucleon reaction dynamics. Precision cross section and polarization measurements of neutron-proton and neutron-deuteron scattering are helping in the refinement of modern NN force models. Our studies also address tensor force effects (with polarized targets), charge-symmetry and charge-independence breaking in the NN force, three-nucleon force effects, and relativistic effects in nuclear reactions. Our collaboration at CEBAF is planning to make detailed studies of the structures of nucleons and nuclei at short distances. 
Physics Department

Durham, North Carolina 27706

Work Performed at the Triangle Universities Nuclear Laboratory (TUNL)

Title of Project: Neutron-Induced Reaction Studies and Nuclear Data Compilation

Person in Charge: N. R. Roberson

Telephone: $\quad$ (919) 660-2600

Scientific Staff: $\quad$ C. R. Howell, W. Tornow, R. L. Walter, H. R. Weller, D. R. Tilley (NCSU)

FY 1992 Funding: $\quad \$ 156,000$

Neutron-Induced Reaction Studies Many areas of applied physics rely on knowledge of how neutrons interact with various materials, ranging from stainless steel and concrete to biological tissue. The knowledge of the interaction comes from measurements, when possible, or from neutron-interaction models when measurements are not possible or are unavailable.

The experimental phase of the neutron program at TUNL concentrates on measurements in the 5- to $20-\mathrm{MeV}$ energy range and determines the interaction probability for both polarized and unpolarized neutron beams. The theoretical modeling phase of the program relies on available data over the entire energy range up to $100 \mathrm{MeV}$. In fact, the dispersive optical model that we are developing also allows one to predict how the neutrons and protons are organized within the nucleus, thereby :acting the basis of our most fundamental pictures of the nucleus.

In the past 2 years, measurements and analyses have been performed on ${ }^{1} \mathrm{H},{ }^{2} \mathrm{H},{ }^{12} \mathrm{C},{ }^{27} \mathrm{Al},{ }^{28} \mathrm{Si},{ }^{32} \mathrm{~S}$, ${ }^{59} \mathrm{Co},{ }^{208} \mathrm{~Pb}$, and ${ }^{209} \mathrm{Bi}$. The high accuracy measurements on hydrogen and carbon allow precise neutron detector calibration. Our broad characterization of the neutron interactions with carbon is expanding our knowledge of radiation damage in human tissue.

Nuclear Data Evaluation TUNL is responsible for data evaluation for the nuclei in the mass region $A=3-20$ in accordance with the recommendation to DOE by the Panel on Basic Nuclear Data Compilations of the National Research Council. This project under the direction of H.R. Weller and D.R. Tilley first performed a review for $A=3$ nuclei. More recently, a review of $A=4$ was done in collaboration with G.M. Hale of LANL. The project for evaluation of nuclear data in the mass range $A=5-20$ carried out for many years by Fay Ajzenberg-Selove at Pennsylvania was successfully transferred to TUNL during 1990. The review of $A=16,17$ nuclei is in progress. Following completion of the $A=16,17$ review, TUNL will begin on $A=18-20$ and continue the cycle. 
Title of Project: Nuclear Physics at Extreme Energy Density

Person in Charge: $\quad$ B. Müller

Telephone: (919) 660-2570

FY 1992 Funding: $\quad \$ 249,(0)$

Nuclear physics is entering an era of investigations focusing on the quark-gluon substructure of nuclear matter. These studies have the same importance for nuclear physics as investigations of atomic and molecular substructure have for chemistry and condensed matter physics. The study of quark matter at extremely high energy density, which is the primary goal of RHIC, will allow us, morecover, to probe the influence of the structure of the vacuum state on the fundamental laws of physics.

Two important theoretical problems in this context are (1) the need to 'identify a reliable set of signatures for the change in the structure of nuclear matter from hadrons to a plasma of free quarks and gluons and (2) a better understanding of the mechanism by which local thermal equilibrium is established in nuclear collisions at very high energy.

We have approached the thermalization problem in the context of perturbative QCD, describing the initial phase of a nuclear collision as a transport problem among quarks and gluons. Our results indicate that there is a direct route from the initial parton substructure of the colliding nuclei to a locally thermalized quark-gluon plasma that can be described quantitatively by our methods. We also found that pre-equilibrium process can be important for reliable predictions of some quark matter signatures, such as lepton-pair and charm production, improving chances to detect these signals experimentally.

Recently, we began nonperturbative investigations of nonequilibrium phenomena in the framework of lattice gauge theory. Our studies, so far, have revealed the universal presence of chaotic dynamics of the nonabelian gauge field, providing a new technique to calculate the thermalizatio a time. Future work will focus on quantitative, parameter-free predictions for quark-gluon plasma signatures and nonperturbative calculations of gluon dynamics.

Since the fall of 1991, the institution of a weckly Triangle Nuclear Theory Collocpuium between Duke, North Carolina State University, and University of North Carolina has fostered the regular communication and exchange of ideas among the nuclear theory groups at the three major universities in North Carolina. 
Supercomputer Computations Research Institute

Tallahassee, Florida 32306

Title of Project: $\quad$ Experimiental Nuclear Physics at the Supercomputer Computations Research Institute

Person in Charge: P. Dragovitsch

FY 1992 Funding: $\quad \$ 100,000$

The CEBAF Large Acceptance Spectrometer (CLAS) currently being built at CEBAF will offer unique opportunities for new types of experiments at medium energies due to the increased complexity of observable physical events made possible by the large acceptance of CLAS and the high beam current available at CEBAF. The acquisition, storage, reconstruction, and analysis of the experimental data collected at CLAS will strain the capabilities of traditional nuclear physics software tools, hecause only more sophisticated approaches, including state-of-the-art phyśics, computer hardware, and computing techniques, can guarantee the successful completion of experiments at CEBAF. The experimental nuclear physics group at the Supercomputer Computations Research Institute (SCRI) has assumed the primary responsibility for CLAS on-line and off-line software. Our efforts also include the participation in numerous proposals for experiments at CLAS.

Our research is currently concentrated on the improvement of detailed computer simulations using GEANT due to the preparation of approved experiments and the calibration of CLAS, on the development of the on-line and off-line data analysis systems for CLAS, and on the development of the CLAS experiment database. Significant contributions to the future design of the data acquisition system of CLAS have been made. Recently, the CLAS distributed event analysis shell has been completed, and a prototype of th: CLAS experiment database already exists.

Our future work will focus on the integration of the experiment data analysis modules into the analysis shell and on testing the analysis algorithms using simulated detector events. We will use the several compute clusters at SCRI to run the analysis code in a massive parallel mode, so testing and optimizing the event distribution and analysis in an environment comparable to the future analysis hardware planned at CEBAF. We will continue to be involved in the development of the on-line and off-line data analysis systems and the data acquisition system for CLAS, in providing support for CLAS Collaboration in their computational needs, and in our active participation in approved experiments at CLAS through computer simulations of the detector and the physics of interest. 
Department of Physics

Tallahassee, Florida 32306

Title of Project: Research in Theoretical Nuclear Physics

Person in Charge: $\quad$ D. Robson

Telephone:

(904) 644-1767

Scientific Staff: $\quad$ A. G. Williams, A. Szczepaniak

FY 1992 Funding: $\quad \$ 110,000$

The major thrust of this research lies in understanding and describing nuclei in terms of quark and gluon degrees of freedom. The physics issues are at the interface of nuclear and particle physics and require parallel efforts in fundamental level theory and model building. The basic underlying symmetries of QCD, such as Lorentz covariance, gauge invariance, and chiral symmetry, play a central role in these studies as does the issue of quark and gluon confinement. In particular, calculations and models that have relevance for CEBAF (and later RHIC) are being pursued.

Current research efforts include studies of the SU(N) gauge group, with emphasis on studies of glueballs in SU(3) using Hamiltonian lattice gauge theory. In addition, the important issues of dynamical chiral symmetry breaking and confinement are being studied through the solution of sets of coupled integral equations. The previous numerical calculations require extensive computational work, and significant use is made of supercomputers.

Relativistic chiral quark models have been formulated for single-hadron systems and have enjoyed some success. Further refinements are being developed, and calculations that apply such models to multihadron systems (e.g., nuclei) are currently in progress. Variational and relativized sudies of few nucleon systems are also being pursued. Recent work has included calculations, of phi-meson production at CEBAF, which show that this is a potentially sensitive probe of the strangeness content of nucleons and nuclei. Further calculations involving the production of strangeness in the nucleus and of nucleon resonances are planned and are crucial to complement the significant experimental efforts in these areas at CEBAF. 
Title of Project: Medium Energy Nuclear Physics

Person in Charge: $\quad$ B. L. Berman

Telephone:

(202) 994-7192

Scientific Staff: $\quad$ K. S. Dhuga, P. L. Cole, S. L. Rugari

FY 1992 Funding: $\quad \$ 208,000$

The central theme of our experimental program in medium energy nuclear physics is the delineation of the properties and symmetries (or the lack thereof) of the many-body nuclear force. The experiments are conducted at several major accelerator laboratories, mostly in the U.S., and the experimental program will focus on CEBAF when it becomes operational in the mid-1990s.

We compare the results of measurements made with both electromagnetic and hadronic probes. The major components of this program have been measurements of electron and pion scattering from nuclei, particularly studies of few-body nuclei, and instrumentation and operation of The George Washington University (GWU) Nuclear Detector Laboratory, both for the preparation of experimental apparatus and for the training of students in nuclear instrume tation techniques.

Recent research results have included measurements of electron scattering at the Bates accelerator at Massachusetts Institute of Technology and the National Institute for Nuclear Physics and High Energy Physics-Amsterdam (NIKHEF) accelerator in Amsterdam and of pion scattering at LAMPF. Electronscattering coincidence experiments, where an ejected particle is detected simultaneously with the scattered electron, have proven effective in measuring the momentum distributions of constituents inside the ${ }^{6 i}$ and ${ }^{12} \mathrm{C}$ nuclei. Pion-scattering measurements on ${ }^{3} \mathrm{H}$ and ${ }^{3} \mathrm{He}$ have shown an apparent breakdown of charge symmetry in these simple three-body nuclei.

Current efforts are centered on two large collaborative projects: the High-Acceptance Recoil Polarimeter (HARP) for use in (e,e'n) measurements, first at NIKHEF and eventually at CEBAF, and the focal-plane detector array of the photon tagger for the CEBAF Large Acceptance Spectrometer (CLAS). Our particular interest in the lightest nuclei is manifested in our leading role in proposals for $\left(e, e^{\prime} n\right)$ and photoreaction measurements on the ${ }^{3} \mathrm{He}$ nucleus.

A suite of rooms in the Physics Building at GWU has been renovated for use as our Nuclear Detector Laboratories, which has been in use for 3 years. This year, GWU has made available to us extensive additional laboratory space and machine tools at our new Virginia Campus. Our Virginia laboratory will be devoted primarily to the photon tagger for CLAS, while our Washington laboratory will continue to serve for the development of HARP and as our primary training facility for students. We also have been successful in obtaining substantial amounts of electronic equipment, computers, and peripheral components, as well as other laboratory instrumentation, particularly with the 1990 award of a DOE University Research Instrumentation Program Grant in support of the HARP project. 
Department of Physics

Washington, DC 20052

Title of Project: Investigations of the Structure and Electromagnetic Interactions of Few-Body Systems

Person in Charge: $\quad$ D. R. Lehman

Telephone:

(202) 994-6486

Scientific Staft: H. Haberzettl, H. Ito, L. C. Maximon, W. C. Parke

FY 1992 Funding: $\quad \$ 200,000$

The George Washington University nuclear theory group investigates the structure and electromagnetic interactions of few-nucleon systems. The properties of the very light nuclei are examined by developing theoretical models that begin from the basic interactions between the nucleons and that are solved numerically using full three- or four-body dynamics. Such models are then used to understand the details of the strong and electromagnetic interactions of few-nucleon nuclei after the basic underlying reaction mechanisms are understood with simpler models. These efforts are increasingly important with the advent of high duty cycle, high current electron accelerators such as those under construction at CEBAF (Newport News, VA) from which data will be forthcoming to allow unraveling the structure and interactions of the very light nuclei.

Current research topics include the following: (1) calculation of pion-nucleon scattering within the relativistic cluster-dynamical approach; (2) investigation of four-nucleon rearrangement scattering, including photodisintegration of ${ }^{4} \mathrm{He}$; (3) theoretical analysis for a photon tagging system at CEBAF; bremsstrahlung cross sections in spectrometer coordinates; (4) calculation of polarization observables for radiative capture of polarized deuterons on hydrogen; (5) examination of the D-state properties of the ${ }^{4} \mathrm{He}$ nucleus; (6) development of the theory for calculation of the observables in electrodisintegration of ${ }^{3} \mathrm{He}$ with exact three-body dynamics; and (7) derivation of the gauge-invariant Compton amplitude for relativistic composite systems.

Of considerable interest at this time is the significant progress made in interpreting theoretically the measured polarization observables for radiative capture of polarized deuterons on hydrogen. Among the major findings are the following discoveries. (1) The initial state of the reaction must he handled with full three-body dynamics in order to be able to interpret the magnitude of the ohserved tensor polarizations at low energies (energies of $20 \mathrm{MeV}$ or less for the deuteron). In other words, neglecting the interaction between the incident deuteron and the target proton is a poor approximation. (2) As one moves upward in energy (for example, $100 \mathrm{MeV}$ deuterons), it may he naively expected that the role of initial-state interaction between the deuteron and proton will diminish based on the differential cross section results. However, this is not the case for the tensor analyzing powers where initial-state rescattering not only can change the magnitude by more than a factor of 2 but also can change the sign. (3) A hierarchical approach to various approximations made in treating the initial-state interactions by numerous investigators is used to show that each one successively moves the theoretical predictions for the tensor analyzing power $T_{20}$ further from the data, clearly indicating their 
inadeyuacy. (4) Of all the components making up the ${ }^{3} \mathrm{He}$ wavefunction, it is the D-state that is totally responsible for the character of the tensor analyzing powers, although there is little sensitivity to the percentage D-state in the deuteron generated by the nucleon-nucleon interaction models considered.

During the coming years, emphasis by the GWU theory group will he given to developing relativistic theories for few-body systems applicable to electromagnetic processes. One aspect of this emphasis is already well underway, namely calculation of pion-nucleon scattering using the cluster-dynamical approach. Significant progress is expected in this work during the next 2 years. 


\section{UNIVERSITY OF GEORGIA}

Department of Physics and Astronomy

Athens, Georgia 30602

Title of Project: $\quad$ Studies of the Spin-Isospin Response of the Nuclear Continuum Using Intermediate Energy Hadrons

Person in Charge: $\quad$ F. T. Baker

Tolephone: $\quad$ (706) $542-2485$

FY 1992 Funding: $\$ \$ 70,000$

The nuclear continuum refers to states in which an atomic nucleus may exist but which are quite highly excited. The challenge in trying to measure and understand the properties of these states is that there is a multitude of possible properties all occurring at nearly identical places in the continuum; thus, unlike less highly excited regions of the nuclear spectrum where one can acquire data for a single state, one is forced to look at many kinds of nuclear states simultaneously in the continuum. One must therefore devise techniques that can "filter" out desired properties of the continuum so that contributions from individual types of states can be isolated. If one is successful in isolating such a type of state, the "response" of the nucleus in this mode is said to have been determined. These types of studies are the main focus of the research supported by this grant.

The experimental measurements are done primarily at three laboratories: LAMPF at LANL, the Tri-University Meson Facility (TRIJMF) in Vancouver, Canada, and the SATURNE synchrotron in Saclay, France. At LAMPF and TRIUMF, studies are carried out by bombarding the targets with intermediate energy protons, the scattered particles being detected and analyzed. At SATURNE, studies are carried out by bombarding targets with intermediate energy deuterons. Using deuterons or protons is a technique for isolating the isospin response of nuclei because deuterons excite oniy isoscalar modes in the nucleus whereas protons excite mainly isovector modes. (An isoscalar mode is one in which constituent neutrons and protons in the target nucleus move in concert, whereas in isovector modes, the protons and neutrons move in opposite directions.) At all three laboratories, we also measure the direction of the spin of 'he scattered particle, and measurements are made at a variety of angles. The measurement of spin per nits a determination of the spin response of the nucleus, and having data at several angles allows on : to determine the composition of the angular momentum of the excited target nucleus.

Recent results include publications in which the nuclear continuum is decomposed into its spin, isospin, and angular momentum components for several nuclei.

Future work will pursue the use of these new techniques to provide more accurate and more detailed decompositions of experimental data. The experiments with deuterons, particularly, are still fertile territory for research. Work will also be done at the new CEBAF when it is completed. 


\section{GEORGIA INSTITUTE OF TECHNOLOGY}

\section{School of Physics}

Atlanta, Georgia 30332

Title of Project: $\quad$ Nuclear Structure from Radioactive Decay

Person in Charge: J. L. Wood

Telephone: $\quad$ (404) 894-5262

FY 1992 Funding: $\quad \$ 90,000$

One of the fundamental challenges in nuclear physics is the testing of our theoretical description of luw energy nuclear structure under conditions of extreme instability. There are two major reasons for this: first, all of our theories are phenomenological and are based on phenomena observed in stable or near stable nuclei; second, it is the nuclei at the extremes of instability that are telling us about new properties of nuclear matter at low energy. The low energy properties determine whether a particular nucleus can exist. The key to testing theories of nuclear structure in nuclei far from stability is systematic and detailed study of many nuclei in selected regions. This is the major objective of this program.

Research is currently focused on the role that the nuclear shape plays in determining nuclear stability and specifically on nuclei that can take on more than one shape, termed "shape isomerism": or "shape coexistence." Experimental measurements are conducted primarily at the University Isotope Separator Oak Ridge (UNISOR) at the Holifield Heavy-Ion Research Facility. The region selected for study is the very neutron-deficient isotopes of platinum, gold, mercury, thallium, lead, bismuth, polonium, and astatine. This region is the most extensive region known that exhibits shape coexistence at low energy.

A recent development is the formulation of a simple theoretical description of shape coexistence, based on the idea that nuclear shapes are controlled by the number of protons and neutrons outside the closed energy shells and by the force acting between them. This description is now being tested and refined.

Future work will focus particularly on electric monopole transitions and their relation to shape coexistence and shape coexistence along the $\mathrm{N}=\mathrm{Z}$ line. 


\section{GEORGIA STATE UNIVERSITY}

Department of Physics and Astronomy

Atlanta, Georgia 30303

Title of Project: $\quad$ Research in Heavy-Ion Nuclear Physics

Person in Charge: $\quad$ G. A. Petitl

Telephone: (404) 651-3221

Scientific Staff: $\quad$ X. T. Liu

FY 1992 Funding: $\quad \$(2,()()$

When RHIC is completed, it will be the premiere facility in the world for research in Relativistic Heavy-lon Nuclear Physics. A number of collaborations is developing detector systems to probe the properties of the "quark-gluon plasma," the new phase of nuclear matter that is expected to be crealed hy this new particle accelerator. One way of probing this plasma is hy observing the leptons resulting from the decay of short-lived vector mesons that are produced in the plasma. This is the basis for the PHENIX detector system, one of the two large experiments that have received funding to be installed and ready to take data as soon as the accelerator becomes operational. Work on the design and development of the muon detection system for the PHENIX detector is the major objective of the Georgia State program.

The work is focused on development of a "muon identifier," a large calorimeter-absorher detector designed to detect and identify muons that are emitted in the decay of vector mesons in the plasma. The "identifier" must also identify and reject pions that are also emitted copiously in the relativistic reactions and that mimic the muons. Effort is divided hetween computer simulations of the performance of the identifier and experimental work using the proton beam at the Brookhaven AGS (o) measure the performance of candidate designs.

Work over the coming year will be devoted to development of a "limited streamer mode" detector system that will he tested at Brookhaven in early 1993. 
Department of Physics

Hampton, Virginia 23668

Title of Project: CEBAF Drift Chamber Development

Person in Charge: $\quad$ O. K. Baker

Telephone: $\quad$ (804) $727-5820$

FY 1992 Funding: $\quad \$ 63,000$

Most of the nuclear matter that exists in our world is made up of up (u) and down (d) (constituent) quarks and gluons. Systems with strangeness are composed of, in addition to $u$ and d quarks, strange (s) constituent quarks. The study of nucleon and nuclear systems with strangeness is one of the forefront areas of research in intermediate energy nuclear physics. The experimental facilities available at CEBAF are ideally suited for study of such systems. The present research effort is to huild the part of the detection system to be used at CEBAF to implement the study.

The goal of this experimental program is to study the electroproduction of kaons at CEBAF using the High Momentum Spectrometer (HMS) to detect the scattered electrons and the Short Orbit Spectrometer to identify the electroproduced kaons. The electrons and kaons will be detected in coincidence. The present program is to design and build the drift chambers to be employed in HMS. These chambers will be used to determine the momenta of the scattered electrons. There will be two (plus one spare for a total of three) chambers built for HMS. Several other experiments have heen approved or conditionally approved at CEBAF, which use HMS and its associated detector subsystems. The HMS drift chambers are an essential part of all of these experiments.

The design of the chambers and prototype development has been completed. The first chamber has been completed and is now operational. The stringing of the second chamber is approximately 70 percent complete. The third chamber should be completely strung by the end of the 1992 calendar year. All three chambers should be operational by the spring of 1993, well ahead of schedule for integration into the HMS detector stack.

The experiment described previously should he ready for data-taking early in the CEBAF program, as soon as heam is available. The future plans are to initially study kaon electroproduction using proton and deuteron targets. Extension to heavier targets will follow. 
Department of Physics

Houston, Texas 77204

Title of Project: $\quad$ Selected Problems in Experimental Intermediate Energy Physics

Person in Charge: $\quad$ E. V. Hungerford

Telephone:

(713) 743-3549

Scientific Staff: $\quad$ K. Lan, B. Mayes, R. Phelps, L. Pinsky, M. Barakat

FY 1992 Funding: $\quad \$ 818,000$

The research project is an experimental investigation into the fundamental interactions between nuclear particles. The goals are to investigate forefront problems in physics at intermediate energies, train students in this field of research, and develop the instrumentation needed to carry out the research program. The work uses the nucleus as a medium in which to study how the many-hody nature of a strongly interacting system influences the fundamental interactions of physics.

We are presently involved in the development of the experimental apparatus for the detection of an electroweak decay, which is not allowed by the Standard Model but could occur in various extensions to this model. This experiment will, if the decay is not observed, provide a limit about 200 times better than the existing one. We plan to hegin a short data run next summer.

We are also involved in an experiment using incident beams of kaons and pions to implant a strongly interacting particle that has an additional degree of freedom, called strangeness, in the nucleus. This research should help clarify the fundamental symmetries of the strong interaction and may provide a mechanism to study quark degrees of freedom in the nucleus.

In the future, we are proposing to construct a high resolution spectrometer to study strange nuclear physics produced by the electromagnetic interaction at CEBAF. This offers the possibility of measuring the transition form factor and thus the nuclear wavefunction to specific levels within the nucleus. 
Title of Project: Theoretical Studies in Medium Energy Nuclear and Hadronic Physics

Person in Charge: $\quad$ M. H. Macfarlane

Telephone: (812) $855-2953$

Scientific Staff: $\quad$ S. Gardner, D. K. Griegel, C. J. Horowitz, T. Matsui, B. D. Serot, W. Unkelbach

FY 1992 Funding: $\quad \$ 280,000$

Our research deals with the structure of nuclei and with the interpretation of experiments designed to probe that structure. It falls into six categories.

(1) Relativistic theories of the structure of nuclei based on meson and baryon degrees of freedom.

(See 5,6). Recent work seeks to include pionic contributions and focuses on the nuclear equation of state at extreme temperature and density.

(2) Studies of spin observables in proton-induced reactions on nuclei at 50 to $800 \mathrm{MeV}$. Nonrelativistic and relativistic models have been used to analyze experimental results from accelerators at Indiana University Cyclotron Facility (IUCF), Los Alamos (LAMPF), and Vancouver (TriUniversity Meson Facility.)

(3) Studies of the properties of hot, dense hadronic matter. We deal with various aspects of the planned experimental program at the Brookhaven RHIC, in particular, suppressed charmonium production as a signal of the formation of quark-gluon plasma.

(4) Suppression due to Coulomb screening of neutrino cross sections in dense matter. This may have significant impact on neutrino propagation in supernovas.

(5) Theories of the structure of nuclear matter based on the underlying quark degrees of freedom. Monte-Carlo simulations for ground-state properties have been carried out for a model that has quark confinement built into it.

(6) QCD sum rules in the nuclear medium are being explored as a way of connecting the observed meson-baryon degrees of freedom of nuclei to the underlying quark-gluon substructure. 
Title of Project: Characterization of the Multifragment Decay Channel in Lighly Excited Nuclei

Person in Charge: R. de Souza

Telephone: $\quad$ (812) $855-3767$

FY 1992 Funding: $\quad \$ 6(0,()()$

At high excitation, nuclear matter decays preferentially into multiple fragments. This decay mode might signal a first order phase transition. Investigation of the nuclear malter phase diagram at extremes of temperature and pressure is essential if phenomena such as neutron stars or supernova explosions are to he understood.

Multifragmentation might arise from either dynamical fluctuations or statistical decay of the excited system on a short timescale. One focus of the research program is therefore a determination of the size and lifetime of the emitting source and, in particular, the dependence on increasing excitation energy. Recent investigation of two fragment correlations revealed the mean fragment emission timescale is of the order of $\tau<10() \mathrm{fm} / \mathrm{c}$. With increasing incident energy, the spatial-temporal extent of the emitting source decreased for the reaction ${ }^{36} \mathrm{Ar}+{ }^{197} \mathrm{Au}$ at $\mathrm{E} / \mathrm{A}=50,8()$, and $110 \mathrm{MeV}$.

Also under investigation is the role of compression in multifragmentation. Experimental fragment multiplicities observed in intermediate energy heavy-ion reactions can only he understood if the density of the emitting system is suhstantially reduced $\left(\rho=0.3 \rho_{t)}\right)$ from its equilibrium value. Such a reduction of the density might occur due to either a dynamical compression-decompression cycle or the thermal pressure of the system. Recent results indicate that the expansion of the emitting system can be prohed hy examination of the mass dependence of the mean transverse kinetic energy due to the velocity shift introduced by surface emission from an expanding source. Comparison of experimental data with a theoretical model might yield insight into the role of expansion. Systematic: comparison of mass asymmetric entrance channels with more mass symmetric reactions is necessary.

In the future, work will focus on a more detailed comparison hetween multifragmentation as a function of entrance channel mass asymmetry. Examination of observables such as the isotopic ratios of emitted fragments may also yield insight into the breakup process. 
Title of Project: Nuclear Reaction Mechanism Studies at Intermediate Energies

Persons in Charge: V. E. Viola, K. Kwiatkowski

Telephone:

(812) $855-2878$

Scientific Staff:

J. Brzychczyk

FY 1992 Funding: $\quad \$ 221,000$

Current research is concerned with formation dynamics and decay mechanisms of hot nuclear matter. Such systems are formed in the collision of energetic nuclear projectiles with complex target nuclei and are currently being studied using nuclear particle accelerators at Indiana University, CEN Saclay, and Michigan State University.

As the relative energy hetween colliding nuclei increases, reaction times become shorter and the role of the individual particles that make up the nucleus becomes increasingly important. In this intermediate energy regime, we ohserve the emission of unusually energetic light particles and complex nuclear fragments (atomic numbers - 3-20) that appear to originate from sources with very high temperattres, much hotter than expected for a nucleus in thermal equilibrium. These nonequilibrium events may signify the existence of nuclear "hot spots"--localized regions of excitation within a nucleus. At sufficiently high projectile energies, these processes may lead to the formation of multiple clusters, or multifragmentation. Such events may signal the vaporization of nuclear matter. Recently, we have performed studies that suggest the heating effects of meson resonances may produce such an effect, causing the nucleus to expand rapidly and undergo subsequent multifragmentation.

To study these phenomena more quantitatively, our group is presently constructing a multiple detector array: The Indiana Silicon Sphere' (ISIS). ISIS will contain 162 detector telescopes that will permit simultaneous measurement of the angular and energy relationships of all charged nuclei emitted in violent nuclear collisions. With this device, we will he able to reconstruct the event pattern for each reaction and thereby gain insight into the behavior of hot nuclear inatter. The results will then be employed to examine predictions of the active theoretical work in this area.

In addition, our group maintains an interest in studying nuclear reactions of astrophysical significance, such as the origin of the chemical elements and cosmic-ray-related phenomena. These measurements have also proven to be important in evaluating the effects of cosmic-rays on hiological systems and microelectronic components during space travel. 


\section{UNIVERSITY OF IOWA}

Department of Physics and Astronomy

lowa City, lowa 52242

Title of Project: Configuration Space Faddeev Calculations

Persons in Charge: G. L. Payne, W. H. Klink, W. N. Polyzou

Telephone: $\quad$ (319) 335-1689

FY 1992 Funding: $\quad \$ 134,000$

It is now generally believed that the particles making up the atomic nucleus, protons and neutrons, are themselves made up of more fundamental particles called quarks; nevertheless, many properties of nuclei can be explained with the conventional model, which assumes that protons and neutrons are the basic particles. The goal of our research is to determine under what circumstances the conventional model can be used to explain data from experiments involving the scattering of particles such as photons, electrons, and protons off of nuclei and to understand which experiments might be sensitive to the underlying quark substructure of the nucleus.

Our research involves the formulation and solution of mathematical models of systems of a small number of particles. Few-body systems are simple enough to model with realistic mathematical models that can be solved to within experimental accuracy on computers. (They are still sufficiently complicated to require the use of supercomputers). Comparison of the results of these model studies with experimentation provides a very direct test of the mathematical models.

A major component of our research is a syst matic investigation of the limits of conventional models of the few nucleon systems. The need for quar' degrees of freedom can only be established if it can be shown that the underlying physics cannot ye understood using conventional methods. We are continuing our extensive investigation of properties of the three nucleon system with conventional interactions.

The scattering of a high current of energetic electrons from the protons and neutrons of the nucleus can be used as a microscope that can resolve the quark substructure of the individual protons and neutrons. A proper interpretation of the results of these experiments requires a realistic relativistic model of the nucleus and a constituent relativistic model of the electron current. The second major component of our research is the construction of relativistic models of few-quark systems and consistent models of the quark current operator for these systems. A recent advance made by our group was a relativistic three-quark model of the nucleon. When combined with a consistent model of the quark current operator, these solutions can be used to predict the results of many important few-body experiments proposed at CEBAF. 
Department of Physics and Astronomy

Ames, lowa 50011

Title of Project: Relativistic Heavy-Ion Physics

Persons in Charge: J. C. Hill, F. K. Wohn

Telephone: $\quad$ (515) 294-6580

FY 1992 Funding: $\quad \$ 197,000$

The major emphasis is to participate in the search for the quark-gluon plasma (QGP) and other forms of hot hadronic matter using RHIC now under construction at BNL. We are members of the PHENIX Detector Collaboration and have a major responsibility for developing the PHENIX triggering system. The triggering will be designed through a collaboration with the Ames Laboratory Instrumentation Group and physicists from Columbia University. Our physics interest is in measuring dilepton spectra in heavy-ion collisions to study the production of the $\phi$ meson and measure its width and branching ratios to characterize hot hadronic matter immediately after the phase transition from QGP. We also plan to study the $J / \psi$ meson from dimuon spectra, which should be a useful probe of QGP.

The relativistic heavy-ion program also emphasizes study of electromagnetic dissociation (ED), whereby a nucleus is excited by photon exchange into a giant resonance. ED processes are predicted to become much larger than nuclear interactions for heavy projectiles at energies projected for the RHIC collider. Measurements at the Bevalac accelerator indicate a three-barn cross section for relativistic ${ }^{238} \mathrm{U}$ projectiles on ${ }^{197} \mathrm{Au}$ and have been extended to ultrarelativistic energies using ${ }^{197} \mathrm{Au}$ beams from the AGS accelerator. Experiments were carried out at the CERN-Super Proton Synchrotron accelerators with ultrarelativistic heavy ions to determine the increase in ED with energy and will he extended to heavier projectiles with the advent of ${ }^{208} \mathrm{~Pb}$ beams. 
Department of Physics

Ames, lowa 50011

Title of Projoct: Intermediate/High Energy Nuclear Physics

Person in Charge: J. P. Vary

Telephone: (51.5) 294-5440

Scientific Staff: J. Qiu, J. R. Spence, M. Luo

FY 1992 Funding: $\quad \$ 160,()(0)$

Theoretical predictions of elementary particle production rates in RHIC form a central focus of these efforts and support the experimiental RHIC program at lowa State University and elsewhere:

The production if photons and of strange and charmed mesons at RHIC will carry potential signals of the hehavior of nuclear matter under the extreme conditions similar to those in the "Big Bang" origin of the universe. We are developing perturbative and nonperturbative approaches, hatied upon QCD, to evaluate these production rates under varying conditions such as projectile-target combination, impact parameter, and collision energy. Our major immediate goal is a detailed description of the production rates from hadron-nucleus collisions where some data already exist for comparison. Extensions to the RHIC domain will then follow.

Our recent work on $J / \psi$ production in hadron-nucleus collisions provides a new mechanism $(0$ understand and to explain the experimentally observed nuclear dependence in high energy scattering involving nuclei. Such understanding is essential for identifying the potential signals of the quarkgluon plasma at RHIC.

Our recent success in explaining the electron-positron coincidence peaks in lower energy heavy-ion collisions provides support for the nonperturhative relativistic few-body dynamics we have developed. We aim to extend these dynamics to describe such systems in the presence of strong external fields similar to those generated by collisions at RHIC. 
Department of Physics and Astronomy

Baltimore, Maryland 2/2/8

Title of Project: $\quad$ Studies of Relativistic Heavy-Ion Collisions

Person in Charge: L. Madansky

Telephone: $\quad$ (410) $516-7381$

Scientific Staff: $\quad$ R. C. Welsh

FY 1992 Funding: $\quad \$ 170,000$

To study the nature of nuclear matter when compressed to high densities via the collision of nuclei, one needs phenomena that are characteristic of this kind of matter. Our twofold program involves the study of emission of electron-positron pairs, and a second program involves a study of hyperons whose spin polarization can be measured and whose magnitude is thought to be characteristic of the medium in which they originate. This follows from the fact that hyperons are thought to be made of quarks where the strange quark comes from the medium.

We have concentrated on experiments searching for the characteristic emission of lepton pairs from i lativistic nuclear collisions produced at the LBL Bevalac. A special spectrometer, termed a Dileptron Spectrometer (DLS), which detects the presence of lepton pairs utilizing characteristic signatures via Cerenkov detectors, has been in continuous use in the past 3 years. Results obtained from both heavy-ion and proton and proton-deuteron collisions have shown clearly that the major source of lepton pairs is bremsstrahlung, radiative production of lepton pairs from mesons produced in these collisions, and a distinct possibility of pion annihilation. We expect the extensive data from the light-ion experiments to form a basis for predicting heavy-ion results through cascade calculations. Deviations would point to phenomena that would be characteristic of the nuclear medium, per se.

A collaboration with BNL has studied hyperons utilizing a TPC as well as detailing of final states of nuclear collisions at $15 \mathrm{GeV} / \mathrm{n}$. A complementary experiment involving stopping antiprotons in nuclei to mimic a localized high temperature system has also been completed in collaboration with Rice University, where the final analysis is being done.

Our group has been active in the new Solenoidal Tracker at RHIC collaboration, anticipating experiments at RHIC. We have made important contributions to simulations, especially those involved with jet physics and jet detection for these experiments because jets act as probes of the medium. 
Title of Project: Research in Heavy-Ion Nuclear Physics

Persons in Charge: S. J. Sanders, F. W. Prosser

Telephone: $\quad$ (913) 864-4626

FY 1992 Funding: $\quad \$ 100,000$

When two atomic nuclei collide together at a sufficiently high energy it is possible for them to fuse into a single compound nucleus. Although the nuclei created are in general exceedingly short lived, by studying how they decay, we can explore the forces responsible for their fleeting stability. The focus of our program is a detailed study in light nuclear systems of one particular decay mechanism corresponding to the compound nucleus breaking apart, or fissioning, into two large fragments. This fission process has only recently become recognized as being important in light systems, and its study is leading to a better understanding of the limits of nuclear stability. An equally fascinating aspect of this process in light systems is the mounting evidence that the final fragments are being emitted with information about the nature of the compound nucleus at the point of its breakup, allowing us to explore the structure of nuclei at very large deformations.

In measurements using the Argonne Tandem Linac Accelerator System at ANL and involving members of its scientific staff, we have developed the experimental systematics of the fission process for various light compound systems. With the use of these results, a model calculation has been devised, based on our current understanding of the relevant nuclear interactions, which is very successful in describing our experimental results as well as similar measurements done by other groups.

The fragments emitted through the fission of light compound nuclei tend to be produced at low temperatures where their quantum states are known. By determining which of these states are preferentially produced, we can learn about the nature of the compound nucleus at the instant of fission. We are now exploring this structural information by measuring the gamma-rays emitted by identified fragments, using high efficiency particle counters that we have developed for this purpose. 
Title of Project: Tiseoretical Interpretation of High Energy Nuclear Collisions

Person in Charge: G. Fai

Telephone: (216) 672-4027

Scientific Staff: $\quad$ D. Seibert

FY 1992 Funding: $\quad \$ 71,000$

High energy collisions of atomic nuclei offer a unique opportunity to create conditions in the laboratory similar to those in certain stars and in the early universe. A theoretical effort parallel to the experimental program (RHIC in the U.S.) is vitally important. A major oujective of this project is a better understanding of nuclear substructure and its manifestations in high energy collisions as well as aspects of the collision dynamics itself over a wide energy range.

We study nuclear matter in the relativistic Hartree approximation to a nonlocal version of the Walecka model containing short distance form factors to simulate an underlying QCD substructure of nucleons and mesons. The re - Iting finite nonlocal field theory modci produces saturated nuclear matter by selecting form factor ranges at the nucleon mass scale. The vacuum contribution is quite small. The effective mass increases and the incompressibility decreases compared to the point-coupling limit.

A major thrust of the project is to advance the interaction of theory and experiment through the analysis of data and the design of future experiments. We suggest a "thermometer" for the temperature of the anticipated transition from the hadronic to the quark-gluon phase. The proposed experimental measurement is the traisverse mass distribution of lepton pairs in the rho-omega peak. The fitting temperature of the experimental spectrum is near the temperature of several models of transition mechanism and collision dynamics. In the $1 \mathrm{GeV} /$ nucleon energy range, we have contributed to the interpretation of the data through a new method that displays high order collective flow effects. The incompressibility in the equation of state of nuclear matter is also studied in terms of momentum-dependent effective interactions.

Future work will focus on questions of the collision dynamics at the lower energies and on more detailed models of the quark-gluon substructure and its manifestations in high energy nuclear collisions. 
Title of Project: Heavy-Ion Collisions and the Nuclear Equation of State

Person in Charge: $\quad$ D. Keane

Telephone: $\quad$ (216) 672-2407

Scientific Staff: $\quad$ M. Justice

FY 1992 Funding: $\quad \$ 113,000$

The overall goal of this project is to study nucleus-nucleus collisions experimentally at intermediate and relativistic energies, with emphasis on measurement and interpretation of correlation effects that provide insight into the possible states of nuclear matter and the quantitative relationship between pressure, density, and temperature over a wide range of conditions of excitation.

During the past year, all the personnel associated with this project, including three graduate students, have reen on leave at LBL and have worked on research full time. Much of this effort has gone into preparing for experiments using the equation-of-state TPC at LBL's Bevalac accelerator; in March 1992, this detector successfully took data in production mode for the first time, and the first physics analyses are now underway.

Prior to the availability of data from EOS, our analysis work focused on a variety of earlier Bevalac experiments; this led to 12 journal papers being published or submitted for publication during the past 12 months. Among these is a paper based on part of the dissertation of one of the supported students in which we measure for the first time the collectivity of the sideward flow correlation (i.e., the fraction of the fragments observed in a $4 \pi$ detector that carry a component of the ordered motion). Previous studies had not excluded a picture in which a relatively small fraction of the participant fragments is responsible for the flow correlation.

We have carried out simulations that help to define the physics performance and engineering specifications of the Solenoidal Tracker at RHIC (STAR) detector for RHIC and have demonstrated the feasibility of measuring Bose-Einstein interference between neutral kaons using STAR. With the closure of the Bevalac scheduled for February 1993, the STAR experiment and NA49 at CERN will be the main focus of future research. 


\section{UNIVERSITY OF KENTUCKY}

Department of Physics and Astronomy

Lexington. Kentucky 40506

Title of Project: Research in Theoretical Nuclear Physics

Persons in Charge: K. F. Liu, T. Draper

Telephone:

(606) $257-4849$

FY 1992 Funding: $\quad \$ 101,000$

An outstanding challenge in theoretical nuclear physics is to understand nucleon structure and interaction from the fundamental theory of constituent quarks and gluons-QCD. Lattice QCD provides a nonperturbative procedure that circumvents the calculational bottleneck via numerical simulation with Monte Carlo methods. The main objective of this program is to calculate various nucleon form factors, wavefunctions, and hadron-hadron interactions to understand nucleon structure and dynamics from first principles.

Present research projects include the study of baryon magnetic moments; electromagnetic transitions; axial-vector, scalar, and pseudoscalar form factors; moments of structure functions; and triple pion correlations of the nucleon. The calculated observables will be compared to the available experimental data. Quark loop effects are being calculated with the $Z_{2}$ microcanonical noise. Thus, we could address the theoretical issues regarding the pion-nucleon sigma term and the proton spin crisis.

For the future, we plan to study finite volume, chiral limit, and dynamical fermion effects to estimate and improve the systematic errors related to the physical quantities we calculate. 
Department of Physics and Astronomy

Baton Rouge, Louisiana 70803

Title of Project: Low Energy Solar Neutrino Measurements: the Soviet-American Gallium Experiment

Person in Chargo: $\quad$ M. L. Cherry

Tolephone: $\quad$ (504) 388-8591

FY 1992 Funding: $\$ \$ 75,000$

A gallium solar neutrino detector is sensitive to the full range of the solar neutrinos from the fundamental proton-proton fusion reaction. Out of a total flux of 132 SNU expected in a gallium detector, $70 \mathrm{SNU}$ is expected from the p-p reaction itself, largely independent of the details of solar model calculations. If neutrino oscillations in the solar interior are responsible for the suppressed ${ }^{8} \mathrm{~B}$ flux measured by the Homestake ${ }^{37} \mathrm{Cl}$ experiment and the Kamiokande water Cerenkov detector, then a comparison of the gallium, chlorine, and water results may make possible a determination of the neutrino mass difference and mixing angle. After 2 years of operatiop, a 60-ton gallium detector in the Baksan laboratory in Russia now sees signals at the level of $58-24 \pm 14$ SNU, where $\xi$ is the ratio of ${ }^{71} \mathrm{Ge}$ extraction efficiency to the measured carrier extraction efficiency. Assuming $\xi=1$, and assuming Mikheyev-Smirnov-Wolfenstein neutrino oscillations, a consistent solution for all three requires $\delta \mathrm{m}^{2} \sim 4 \times 10^{-8}-10^{-5} \mathrm{eV}^{2}$ and $\sin ^{2} 2 \Theta \sim 10^{-3}-1$. A $1 \mathrm{MCi}{ }^{51} \mathrm{Cr}$ source calibration is planned for 1993 to measure the solar neutrino capture efficiency. 


\section{LOUISIANA STATE UNIVERSITY}

Department of Physics and Astronomy

Baton Rouge, Louisiana 70803

Title of Project: $\quad$ A Search for Electron-Positron Pairs Produced in Relativistic Nucleon-Nucleon, Nucleon-Nucleus, and Nucleus-Nucleus Collisions

Person in Charge: $\quad$ P. N. Kirk

Telephone:

(504) $388-5847$

\section{Scientific Staff: $\quad$ Z. Wang}

FY 1992 Funding: $\quad \$ 163,000$

The purpose of this project is to search for electron-positron pairs ( $\mathrm{e}^{-}-\mathrm{e}^{+}$pairs) that are produced directly in interactions between relativistic nucleons and nuclei rather than through some intermediate process such as the Dalitz decay of neutral pions or the Bethe-Heitler conversion of photons within the target or surrounding material. The motivation for this experimental program is that the detection of direct $\mathrm{e}^{-}-\mathrm{e}^{+}$pairs provides an attractive technique for studying the nuclear equation of state and the dynamice of pion interactions within nuclear matter. In addition, if the physical mechanisms responsible for the production of direct $\mathrm{e}^{-}-\mathrm{e}^{+}$pairs can be understood at the energies accessible in existing accelerators, then measurements of the cross section for the production of direct $e^{-}-\mathrm{e}^{+}$pairs will be a useful tool in the search for the quark-gluon plasma (QGP) in later experiments at RHIC.

This experimental program is being carried out on the Dilepton Spectrometer (DLS) at LBL in collaboration with physicists from Johns Hopkins University, UCLA, Northwestern University, LBL, and Universite Blaise Pascal. The DLS Collaboration has already presented evidence for the production of direct $\mathrm{e}^{-}-\mathrm{e}^{+}$pairs in proton-Beryllium and Calcium-Calcium interactions. The range of energies covered in these initial reports was $1.0 \mathrm{GeV}$ per nucleon to $4.9 \mathrm{GeV}$ per nucleon. Results have recently been obtained for Niobium-Niobium interactions, proton-proton interactions, and protondeuterium interactions, and these results will be published in the immediate future.

In addition, the Intermediate Energy Nuclear Physics Group at LSU is a member of the PHENIX Collaboration, which has been formed to search for the formation of QGP at RHIC. The collaboration wishes to examine as many of the potential signatures for the formation of the plasma as possible, but it plans to place special emphasis on the production of dileptons and photons. 
Department of Physics and Astronomy

Baton Rouge, Louisiana 70803

Title of Project: $\quad$ Nuclear Interactions of High Energy Heavy-Ions and Applications in Astrophysics

Person in Charge: J. P. Wefel

Telephone: $\quad$ (504) $388-8696$

Scientific Staff: $\quad$ T. G. Guzik, J. Ishert, C.-X. Chen

FY 1992 Funding: $\quad \$ 128,000$

The interaction of energetic complex nuclei with matter is a fundamental investigation whose results are important to diverse fields-radiation damage and shielding, biophysics and radiation therapy, space science, and astrophysics-yet the underlying nuclear processes are only moderately well understood. The goal of this project is to investigate the mechanisms of fragmentation of projectile nuclei in collisions with a variety of targets for different nuclear heams. Current data and theoretical models suggest that the dominant mechanism involves collective interactions between groups of nucleons at low energies, while at high energies the interaction is between individual nucleons. The important transition region between these two extremes is heing studied here, and the data are providing the information needed to evaluate and extend current models.

Experiments have been performed at the LBL Bevalac heavy-ion accelerator in the Beam 40 area using an isotope identification system made up of silicon solid-state detectors and a drift chamber trajectory detector. These data.include measurements of the momentum, energy, and mass distributions of fragments from'the interaction of ${ }^{16} \mathrm{O}$ projectiles at 170,225 , and $360 \mathrm{MeV} / \mathrm{amu}$ and

${ }^{28} \mathrm{Si}$ projectiles at $245 \mathrm{MeV} / \mathrm{amu}$ with $\mathrm{C}$ and $\mathrm{CH}_{2}$ targets. The single-particle inclusive cross sections obtained from these data show a pronounced energy dependence, and the momentum distribution shows an energy dependence not predicted by current models. Data on the total interaction cross section of ${ }^{28} \mathrm{Si}$ at 375,550 and $1050 \mathrm{MeV} / \mathrm{amu}$ on $\mathrm{H}, \mathrm{C}, \mathrm{Cu}$, and $\mathrm{Pb}$ targets were also obtained. These cross sections also show a pronounced energy dependence of -10 percent to -20 percent hetween the low and high energy values and scale with target and projectile mass somewhat greater than the $1 / 3$ power normally associated with optical model predictions.

We have also performed studies of over 20 beams covering species from ${ }^{4} \mathrm{He}$ to ${ }^{58} \mathrm{Ni}$ over the energy range 400 to $910 \mathrm{MeV} /$ nucleon on a liquid hydrogen target in the Bevalac Beam 42 area during 1990 and 1991 using the Heavy-Ion Spectrometer System facility. The charge changing cross sections show a strong odd-even variation, which depends upon the neutron content of the projectile and indicates that the neutron/proton ratio plays a significant role in the fragmentation of these nuclei. The fragment mass identification has been completed for ${ }^{32} \mathrm{~S}$ at $400 \mathrm{MeV} /$ nucleon and indicates an experiment resolution of $\sim 0.2 \mathrm{amu}$. The single particle inclusive cross sections for ${ }^{32} \mathrm{~S}$ fragments have heen obtained and agree to within $\sim 20$ percent to $\sim 40$ percent with current semi-empirical cross section prediction formula. 
The analysis of this extensive database continucs with the Beam 40) analysis nearing completion and the Beam 42 analysis beginning to enter a "production" mode. Future plans include combining the lower energy Beam 40 results with the higher energy Beam 42 information to provide an extensive study of cross section excitation functions and momentum distribution covering very broad ranges of projectile species and incident energy. Furthermore, data from a high efficiency neutron detector are also available for many of the Beam 42 runs, and we intend to investigate correlations between the neutron and charge particle emissions from the same nuclear interactions. Finally, plans are currently under development for studying the interactions of species with $Z>26$ using a liquid Argon calorimeter. 
Department of Physics and Astronomy

Baton Rouge, Louisiana 70803

Title of Project: The Structure of Nuclei Far From Beta Stability

Person in Charge: $\quad$ E. F. Zganjar

Telephone: (504) 388-5833

FY 1992 Funding: $\quad \$ 110,000$

The Louisiana State University experimental nuclear structure group has carried out studies on nuclei far from stability through the use of an isotope separator on-line to a heavy-ion accelerator where proton-rich nuclei are studied following beta decay and through in-beam spectroscopic measurements on proton-rich, heavy-ion reaction products as they gamma decay from high energy, high angular momentum states. The primary effort was located at HHIRF in Oak Ridge. In addition to providing important information on nuclear structure and the nuclear force, the study of nuclei far from stability enhances the role of the nucleus as a microscopic quantum-mechanical laboratory and in this way contributes directly to fundamental knowledge.

Of the various regions under study, significant results have been obtained from studies in the protonrich region between platinum and lead where nuclear shape coexistence has been demonstrated over the widest range of nucleon numbers. We have shown that a pattern of transitions containing electric monopole components in the odd-mass nuclei in the region bears a close resemblance to the electricmonopole-enhanced transitions in the neighboring doubly even isotopes, and that this electric monopole enhancement is associated with transitions between nuclei with quite different shapes. Further, we have observed essentially pure electric monopole transitions in an odd-mass nucleus. This is unprecedented.

Recent innovations include the development of an internal-pair-formation spectrometer to observe electric monopole transitions at high energy. This spectrometer was utilized to determine if beta decay might populate the superdeformed band observed via in-beam spectroscopy in several neutron-deficient mercury isotopes. A picosecond lifetime measuring system was also developed to determine the lifetime of excited $0^{+}$states, which decay only by internal conversion.

Immediate future work will be directed at the clarification and quantification of the electric monopole enhancement observed between shape coexisting nuclear configurations. Longer term future work will involve the use of recoil mass separators and radioactive nuclear beams to extend these studies to nuclei even farther from stability and to utilize the spectroscopy associated with exotic decay modes. 
Titlo of Project: Theoretical Studies in Hadronic and Nuclear Physics

Person in Chargo: J. J. Griffin

Tolephone: $\quad$ (301) 405-6118

Scientific Staff: $\quad$ M. K. Banerjee, T. D. Cohen, H. Forkel, D. B. Leinweber, H. J. Lu, W. M. MacDonald, E. F. Redish, S. J. Wallace

FY 1992 Funds: $\quad \$ 595,000$

The research program of the University of Maryland's Nuclear Theory Group covers a diverse range of topics, each of which is fundamental to our understanding of nuclei. A brief summary of major interests follows.

Hadrons in Nuclear Matter The properties of hadrons propagating in the environment of the nucleus is under active study. QCD sum rules have been developed for finite density systems. Calculations hased on various models of hadrons are also underway. Possible phenomenological signatures of hadronic properties in the nuclear medium are being considered.

The Structure of Hadrons Lattice gauge calculations are being carried out with particular emphasis on baryon properties such as magnetic moments, mean square charge radii, etc. The study of the chiral confining model has entered a new phase with the incorporatior. of instanton effects. An approach to relativistic many-body problems free of pathologies, developer here, is being applied to the problem of baryon structure. QCD sum rules are being used to calculate nucleon properties. The relationship of the chiral perturbation theory to the hedgehog models of baryon structure is under close scrutiny.

Relativistic Nuclear Physics New approaches are being developed to incorporate relativistic dynamics into analyses of two- and three-nucleon systems. Electromagnetic interactions are being studied to develop new techniques applicable to forthcoming data from CEBAF. Relativistic dynamics has been found to be important in proton-nucleus interactions, and work to extend this discovery is being pursued vigorously.

Heavy-Ion Dynamics and Related Processes Our successful "composite particle (quadronium?)" phenomenology for the Gesellschaft fur Schwerionenforschung, Darmstadt (GSI) (e+e-)-puzzle implies bound decays of the composite particle. Consequently, it predicts two otherwise forbidden decay modes (one photon- and sharp annihilative positron-decay) which could validate the bound decay, and two others (bipositron emission, and trilepton emission) which could establish a quadronium (e+e+e-e-) component in the decaying state. These predictions therefore challenge future experiments to speak definitively both to the existence of the composite particle and to its quadronium internal structure. 


\section{UNIVERSITY OF MARYLAND}

Department of Chemistry and Biochemistry

College Park, Maryland 20742

Title of Project: $\quad$ Reaction Mechanism Studies of Heavy-Ion-Induced Nuclear Reactions

Person in Charge: $\quad$ A. C. Mignerey

Telephone: $\quad$ (301) 405-1852

Scientific Staff: J. Shea

FY 1992 Funding: $\$ 205,000$

Understandinn mechanaisms through which heavy ions interact over a broad energy range is the primary goal of this research. Current experimental work is on reactions in the intermediate cuicrgy regime (from $\mathrm{E} / \mathrm{A} \sim 30-100 \mathrm{McV}$ ), where emphasis is being placed on answering such questions as: How much excitation energy can a nucleus hold before it disintegrates into fragments? Is there something analogous to a liquid-gas phase transition for nuclear matter? Looking to the future, RHIC will provide the opportunity to explore properties of another kind of phase transition-that hetween quarks and nucleons. Signatures of the formation of a quark-gluon plasma are being investigated through the group's participation in the PHOBOS Collahoration.

The program in intermediate energy heavy-ion reactions was pioneered at the LBL Bevalac. Using reactions of heavy heams on lighter targets, the emission of two or more complex fragments (defined as nuclei with charges greater than 3) was detected from composite systems with excitation energies up to $8 \mathrm{MeV} / \mathrm{u}$. For the heavier systems $\mathrm{La}+\mathrm{Cu}$ and $\mathrm{La}+\mathrm{La}$ at $\mathrm{E} / \mathrm{A}=45$ and $55 \mathrm{MeV}$, there is evidence for a true simultaneous multifragmentation.

Our work in the intermediate energy regime has now moved to the National Superconducting Cyclotron at Michigan State University. In collaboration with the Westfall group and the 4-pi array, our group has contributed an array of detectors to measure the charge and energy of very forward projectile-like products in coincidence with target-like decay products.

Participation in the PHOBOS Collaboration is a new direction for the group. The PHOBOS detector is a small double-arm spectrometer designed to measure particles with mass up to deuterons at verv low transverse momenta, with excellent momentum resolution and particle identification. Pla-is include experiments at hoth AGS and RHIC. 
Department of Chemistry and Biochemistry

College Park, Maryland 20742

Title of Project: $\quad$ Radioactive Decay and Nuclear Structure Studies

Person in Charge: W. B. Walters

Telephone: $\quad$ (301) $405-1801$

Scientific Staff: $\quad$ H. Penttila

FY 1992 Funding: $\quad \$ 105,000$

The research in this project is directed at the elucidation of new facets of nuclear structure that are important to the testing and development of miodels for nuclear structure and nuclear reactions including fission, for uses in astrophysical calculations, and for applied uses in government and industry. Studies for which beam time is currently approved include: (1) searching for the position and lifetime of pnssibie and predicted superdeformed isomers in the $\mathrm{Hg}, \mathrm{Pb}$, and Po nuclides; (2) ineasurement of the half-life, decay scheme, and $\mathrm{Q}$ value for the decay of Se-68, an $\mathrm{N}=\mathrm{Z}$ nuclide that lies at a crucial point in the rp process for the synthesis in stars of neutron-deficient isotopes of elements up through $\mathrm{Z}=50$; and (3) the study of magnetic moments of nuclides near the double closed shell at Sn-132, which provide direct information of the strength of the shell closure and the interaction of one or more nucleons with that core nuclide.

New work in the future will be aimed at the study of nuclides very far from stability along the proton drop line where masses, half-lives, decay modes, and structure will be determined. Another possible new project will be the measurement of magnetic moments of heavy fission products to support theoretical efforts to describe the fission process.

Recent important measurements have demonstrated the sensitivity of nuclear magnetic dipole moments in both $\mathrm{Br}$ and $\mathrm{Sb}$ nuclides to the shape of these nuclides and configuration of their ground states. Other measurements of lifetimes and conversion coefficients in the Te, I, and Xe nuclides have established the collectivity of these nuclides and challenged the current theoretical description of their structure. 


\section{UNIVERSITY OF MASSACHUSETTS, AMHERST}

Department of Physics and Astronomy

Amherst, Massachusetts 01003

Title of Project: $\quad$ Medium Energy Nuclear Physics

Person in Charge: $\quad$ G. A. Peterson

Telephone:

(413) 545-2008

Scientific Staff: $\quad$ J. Dubach, L. Elouadrhiri, R. Hicks, R. Miskimen, K. Wang

FY 1992 Funding: $\quad \$ 351,000$

This nuclear physics program is a joint experimental and theoretical effort aimed at advancing our understanding of nuclei and their constituent particles, as well as the interactions among the constituents. The primary experimental goal is to attack frontier problems by using the electromagnetic interaction involved in the scattering of high energy electrons from nuclear targets. Subsidiary goals are to design and build the necessary instrumentation for this work, while at the same time providing training for students and visiting scholars.

At this time, we are developing a target for use at the MIT/Bates Linear Accelerator Center, which will contain helium-3 at a pressure of 50 atmospheres and at a temperature of 20 celsius degrees above absolute zero. These conditions are necessary to achieve a high enough density of helium-3 so there is a reasonable probability that an intense high energy electron beam will interact with the nuclei. The detection of electrons scattered from the beam in the backward direction will allow us to explore a shorter range magnetic structure of this three-nucleon nucleus than has been achieved in other experiments.

A measurement has been completed at the Bates laboratory of the threshold breakup of the deuteron where neither the neutron or proton acquire much energy, but the electron transfers a large momentum to them. The results show that conventional theories reasonably agree with the data, but quark cluster calculations show poor agreement.

The interaction of $14.5 \mathrm{GeV}$ electrons and positrons with the nuclei of residual gas atoms in the PEP storage ring of SLAC is being analyzed. We have been joined in this work by Russian physicists from the Institute of Theoretical and Experimental Physics in Moscow. We are also developing apparatus to be used at CEBAF in future studies. 
Title of Project: Neutron-Scattering Studies in the Actinide Region

Persons in Charge: G. H. R. Kegel, J. J. Egan

Telephone: (508) 934-3281

Scientific Staff:

A. Mittler, E. Sheldon

FY 1992 Funding: $\quad \$ 122,000$

The actinides are the heaviest atomic nuclei found in nature and are of great importance to nuclear technologies. These nuclei are ellipsoidal rather than spherical in shape. When they are excited by an external stimulus such as bombardment by neutrons, they are capable of rotation as well as vibration in soveral different modes, or they can break up into much lighter fragments, a process known as fission.

These nuclei, when excited into their rotational or vibrational configurations, have energies that can be classified according to certain characteristic properties of their motion into what are referred to as energy level bands. In the conduct of our research, we utilize our 5-million-volt accelerator to produce neutrons of carefully selected energies with which we bombard actinide nuclei. In neutron-nucleus collisions, some energy is transferred to the bands. By measuring the number and the energy of neutrons after collision, we obtain information that can be used to determine the band structure of the excited actinide nuclei.

The study of the fission process is also of interest. When an actinide nucleus fissions, several neutrons are released which can be used to sustain a chain reaction, provided the neutrons have the proper energy. In this research, we measure the energies of the neutron released in fission when neutrons of different energies initiate the process. The results of the measurements will be compared to theoretical models used to calculate the energies of the released. neutrons, thus providing a test of the models.

Reliable calculations are of considerable practical importance because they enable reactor designers to predict the behavior of nuclear reactors before they are constructed. 


\section{UNIVERSITY OF MASSACHUSETTS, LOWELL}

Department of Physics and Applied Physics

Lowell, Massachusetts 01854

Title of Project: $\quad$ Beta and Gamma Decay Heat Measurements between $0.1-50,000 \mathrm{~s}$ for Neutron Fission of ${ }^{235} \mathrm{U},{ }^{238} \mathrm{U}$, and ${ }^{239} \mathrm{Pu}$

Porsons in Charge: W. A. Schier, G. P. Couchell

Telephone: $\quad$ (508) $934-3770$

Scientific Staff: $\quad$ D. J. Pullen

FY 1992 Funding: $\$ 75,000$

When fuel material in a reactor fissions, releasing large amounts of energy, it generally forms two lighter mass nuclei that are highly radioactive. These fission-product nuclei usually emit both energetic beta particles and gamma-rays when they decay. Because the fuel nuclei fission many different ways, a large variety of fission-product nuclei is formed. These fission products are important in the operation $o^{c}$ a reactor because they produce heat and radioactivity for considerably long times after fission. Dựing the normal operation of a reactor, decay heat contributes to the useful energy extracted from the system. Because the energy release from these products continues even after a reactor is shut down, decay heat must be continuously removed from the reactor by coolant flow. This heat is an important safety issue in cases. where the flow of coolant is interrupted, as may occur in certain types of accidents! Backup cooling systems must be capable of removing the decay heat in order to prevent overheating of the reactor or even core meltdown.

It is important to know the energy released by fission products as a function of time after fission and also to establish how the energies of the emitted beta particles and gamma-rays are distributed. These energy distributions or "spectra" are needed for determining exactly where in the reactor the decay energy is deposited and are also relevant for designing the optimum shielding against this radioactivity. Such energy spectra are generally obtained in one of two ways:

(1) The jeta-particle and gamma-ray spectra for each fission product can be separately measured or esi:mated from theoretical calculations. The contributions from each product are added in what are referred to as "summation calculations," which weight the contributions to account for the production probability of each product in an operating reactor. Summation calculations require an extensive database of fission-product nuclides such as Evaluated Nuclear Structure Data File (ENDF/B-VI).

(2) The second approach is to directly measure the aggregate decay heat from all fission products simultaneously, providing the aggregate sample contains fission products in the same proportion that occurs in nuclear reactors. In this method, the evolution of the spectra with time after fission is needed.

In this project, aggregate decay heat for beta and gamma-rays will be separately measured following the neutron fission of ${ }^{235} \mathrm{U},{ }^{238} \mathrm{U}$, and ${ }^{239} \mathrm{Pu}$ over the delay time range $0.1-50,0(0) \mathrm{s}$. 
Short delay umes $(0.1 \mathrm{~s})$ are made accessible hy mcans of a helium-jet/tape-transport system already developed for use in our earlier delayed neutron studies. Gamma-ray spectra will be measured with a large 5-in x 5-in $\mathrm{NaI}(\mathrm{EI})$ detector for decay heal and with a germanium detector for individual gammaray line intensities. In both cases, the use of hela-gamma coincidence technique provides premium quality gamma spectra by strongly suppressing the background, removing betas and bremsstrahlung, and selecting a precise delay time interval. Furthermore, because no fission foil is present in the counting room, there are no self-absorption effects and no prompt fission contributions due to ambient neutron backgrounds. Likevise, our recently developed beta spectrometer has been demonstrated to give premium beta energy 'spectra for similar reasons. Comparisons with summation calculations using ENDF/B-VI will be performed in parallel with the measurements through a close collaboration with Dr. T. England at LANL assisted hy one of our graduate students during summer residencies at LANL. Our aggregate measurements in the time interval $0.1-20 \mathrm{~s}$ will be of special importance because in thi: region, data from many short-lived nuclides are still missing in ENDF/B-VI, and summation calculations for this region generally rely on model calculations for up to 30 percent of the hear contributions. Several hundred individual gamma line intensities will also be measured as a function of delay time over the range $0.1-50,000 \mathrm{~s}$, providing numerous tests per nuclear fuei for the ENDF/B-VI compilation at the microscopeic, individual-nuclide level. 


\section{MASSACHUSETTS INSTITUTE OF TECHNOLOGY}

Bates Linear Accelerator Center

Middleton, Massachusetts 01949

Thtle of Project: Bates Linear Accelerator Center

Person in Charge: S. Kowalski

Telephone:

(617) 245-6600

Scientific Staff: $\quad$ G. Dodson, J. B. Flanz, K. Jacobs, W. W. Sapp, C. Tschalaer

FY 1992 Funding: $\$ 8,000,000$

The Bates facility supplies very high quality, high intensity, pulsed electron beams. The linear accelerator (202 meters in length) provides beams with energies up to $500 \mathrm{MeV}$ with one pass of acceleration. The electron beam can be recirculated for a second pass of acceleration, thereby increasing its maximum energy to $950 \mathrm{MeV}$ maximum.

The parameters of the primary electron beam are:

(1) maximum operating energy (single pass)- $500 \mathrm{MeV}$;

(2) maximum operating energy (recirculated) $-950 \mathrm{MeV}$;

(3) beam current-100 microamperes (35 microamperes when recirculated);

(4) energy spread-more than 80 percent of current within 0.3 percent;

(5) beam emittance at maximum energy- $0.01 \mathrm{~mm}-\mathrm{mrad}$;

(6) beam polarization- 40 percent; and

(7) duty factor- $<1$ percent.

An energy compression system is in the final stages of construction. It will reduce the electron beam energy spread to 0.04 percent.

There are two major experimental areas. The North Hall contains an energy-loss spectrometer system, which provides the capability for ultrahigh resolution electron-scattering studies of atomic nuclei. A special apparatus is used in conjunction with the spectrometer for $180^{\circ}$-scattering experiments. This capability is complemented in the Scuth Hall by a unique set of three medium resolution, large solid angle, broad range magnetic spectrometers. Presently under construction is a set of four small magnetic spectrometers suitable for out-of-plane measurements, as well as a proton focal plane polarimeter for the largest spectrometer.

A major new thrust is the development of the South Hall Ring Experiment (SHRE), a stretcher/storage ring facility. One of the existing South Hall beamlines will become part of SHRE, providing the capability for a novel set of internal target experiments. Such experiments open up new avenues for research by utilization of ultrathin targets and by provision of a full spin capability for the first time in 
electronuclear physics. In addition, SHRE will be used as a pulse stretcher, providing high duty factor ( $~ 85$ percent) beams to targets on the spectrometer pivot. This will allow new programs involving the simultaneous detection of two or more particles with the existing South Hall spectrometers. The schedule calls for commissioniag SHRE starting in FY 1993. 
Title of Project: Bates Linear Accelerator Center Research

Person in Charge: S. Kowalski

Telephone: $\quad$ (617) 245-6600

Scientific Staff: $\quad$ G. Dodson, K. Dow, M. Farkhondeh, W. W. Sapp, D. Tieger, C. Tschalaer, W. Turchinetz, C. F. Williamson

FY 1992 Funding: $\quad \$ 1,088,000$

The basic research at Bates Laboratory is centered on the use of high quality, high intensity, pulsed electron heams with energies up to $1,000 \mathrm{MeV}$. Operating as an "electron microscope," experiments are designed to exploit the well-known character of the electromagnetic interaction and the precision this tool brings to the study of matter. Structures less than $10^{-13} \mathrm{~cm}$ in extent are resolved.

Experiments map the spatial distribution of nuclear charges and currents and provide stringent tests of mean-field theory and a fundamental understanding of the nuclear force.

In addition to the class of experiments that only measures the momentum of the scattered electron, other studies are concerned with the simultaneous detection of several particles emerging from a collision. Such coincidence experiments are designed to probe the momentum of protons and neutrons in nuclei and to provide detailed information on reaction mechanisms. The goal is to better understand how various phenomena, such as the overlap of interacting quark clusters or the synchronous motion of many protons and neutrons, affect the energies, directions, multiplicity, and identity of the fragments resulting from a collision.

Another area under active investigation is that associated with polarization. Many important physics questions can only be answered by measurements involving polarized beams and targets. A polarized beam has been developed at Bates. The first experiments measuring such fundamental quantities as the charge distribution of the neutron and of the deuteron, the most elementary nucleus, and tests of the electroweak theory have been successfully carried out.

Looking to the future, the South Hall Ring Experiment at the Bates Laboratory is an experimental initiative designed to increase the beam duty factor to essentially 100 percent and to provide an internal target capability. In the internal target mode, the electrons are circulated thousands of times through a windowless gas target introduced into the ring. It will allow us to take advantage of experiments on polarized targets, which are so extremely thin that the standard approaches are impractical. Such internal target measurements, representing a significant departure from the experimental configuration in electronuclear physics, will require innovative developments advancing both physics and technology. A large $4 \pi$ detector has been designed to take maximal advantage of this new capability. This combination of internal targets and polarized beams will be unique in the world. 


\section{MASSACHUSETTS INSTITUTE OF TECHNOLOGY}

Laboratory for Nuclear Science

Cambridge, Massachusetts 02139

Title of Project: Medium Energy Nuclear Physics

Persons in Charge: A. M. Bernstein, T. W. Donnelly, S. B. Kowalski, J. L. Matthews, R. G. Milner, R. P. Redwine, W. Turchinetz, C. F. Williamson

Telephone:

(617) 253-7062

Scientific Staft: $\quad$ R. Ent, W. Korsch, S. Pate, N. Simicevic, P. Welch

FY 1992 Funding: $\quad \$ 1,579,000$

The Medium Energy Nuclear Physics Research Group is involved in a wide range of programs at the MIT-Bates Linear Accelerator Center and at other laboratories. The overall goal of the program, stated most broadly, is to further our understanding of nuclear structure and interaction mechanisms.

Recent experiments at Bates have focused mainly on electromagnetic interactions with few-nucleon systems. For example, a measurement of the tensor polarization of the recoil deuteron in elastic electron-deuteron scattering yielded results which provide stringent tests of nuclear dynamics and quark cluster models.

Spin-dependent electron scattering from polarized ${ }^{3} \mathrm{He}$ was observed in two experiments that used different techniques for polarizing the target. The polarized ${ }^{3} \mathrm{He}$ target development provides the basis for our leading role in a proposed major experiment at DESY (Hamburg), which will measure proton and neutron quark distributions. It has also been used in an experiment at Indiana University Cyclotron Facility, which exploits spin observables in proton scattering.

Our group is heavily involved in the design and construction of the proposed Bates Large Acceptance Spectrometer Toroid (BLAST) detector at Bates, which will use the internal target area of the South Hall Ring for precise measurements of spin-dependent cross sections in electron scattering.

Using the higher energy electron beam available at SLAC, the quasi-elastic (e,e'p) reaction was observed in a range of nuclei from $\mathrm{H}$ to Au. At sufficiently high momentum transfer, these data may exhibit the phenomenon known as "color transparency." Observation of this effect would be an important confirmation of the applicability of perturbative QCD to the quasi-elastic scattering process.

Complementary studies of hadronic interactions have been carried out using pion beams at LAMPF and Paul Scherrer Institute (PSI). Of special interest are reactions in which at least two nucleons must participate, such as pion double-charge-exchange. A systematic survey of this process in nuclei from $\mathrm{C}$ to $\mathrm{Pb}$ has been completed. A more detailed investigation of pion scattering processes, including single- and double-charge-exchange in ${ }^{3} \mathrm{He}$ and ${ }^{4} \mathrm{He}$ is nearing completion. At LAMPF, we are participating in the construction of a new neutral meson spectrometer with large enhancements in energy resolution and efficiency for extensive studies of pion single-charge-exchange in nuclei. 
Experiments on pion absorption have yielded several surprises in the last few years. In particular, a large component of the absorption process seems to involve more than two nucleons and may indicate new physics in the region of nonperturbative QCD. At PSI, a very large acceptance detector system has been constructed, primarily for the purpose of investigating such reactions. Data have recently been obtained on ${ }^{3} \mathrm{He},{ }^{4} \mathrm{He}, \mathrm{N}$, and Ar. 


\section{MASSACHUSETTS INSTITUTE OF TECHNOLOGY}

Laboratory for Nuclear Science

Cambridge, Massachusetts 02139

Title of Project: Studies of Nuclear Interactions Using Electromagnetic Probes

Porson in Charge: W. Bertozzi

Telephone:

(617) $253-5167$

Scientific Staff: $\quad$ J. P. Chen, D. S. Dale, S. Gilad, L. B. Weinstein

FY 1992 Funding: $\$ 877,000$

The research of the Nuclear Interactions Group focuses on the study of electromagnetic currents in nuclei and nucleons as a means towards learning about the fundamental nature of nuclear interactions and nuclear and nucleon structure. Of particular importance are the modifications of the nucleon in the nuclear medium, the large deviation from popular mean-field description of nuclei, and the role played by two- and many-body currents. Results of experiments with the ${ }^{12} \mathrm{C}\left(e, e^{\prime} p\right)$ reaction have demonstrated the inadequacy of the conventional quasi-free knockout concept for the (e,e') reaction and the importance of many, body correlations. These are manifested via anomalous transverse/longitudinal cross sections and large transverse strength at very deep missing energies.

The program of (e,e'p) measurements is being extended to include the deuteron and light nuclei and to include other electromagnetic interference cross sections requiring out-of-plane measurements and polarimetry of the outgoing protons. Most of the experimental program is currently at Bates. The transverse and longitudinal response functions were measured for the deuteron, and the interference response function $\mathrm{R}_{\mathrm{LT}}$ was measured for the deuteron and ${ }^{12} \mathrm{C}$. Out-of-plane detection of the outgoing proton and a polarized electron beam enabled for the first time a measurement of the "fifth" structure function $\mathrm{R}_{\mathrm{LT}}^{\prime}$ of both ${ }^{12} \mathrm{C}$ and the deuteron.

In addition to experimental running, the experimental program at Bates involves the construction of liquid deuterium targets and of the focal plane detection system for the five out-of-plane spectrometer (OOPS) used in the out-of-plane experiments. The group is constructing the multiwire proportional chambers for the focal plane; polarimeter used with One hundred inch proton spectrometer (OHIPS) in a program to study polarization variables in ${ }^{2} \mathrm{H}\left(e, e^{\prime} p\right)$ and other $\left(e, e^{\prime} p\right)$ reactions.

The group has a growing effort at CEBAF. The group is part of the Hall A and Hall B collaborations. In Hall $A$, the group is developing the focal plane detection system for the two high resolution spectrometers. The group is also responsible for target development and beam monitoring in Hall $B$. The approved research plans for Hall A include an extensive program' of (e,e'p) studies with the deuteron, few-body systems, and complex nuclei. In Hall $\mathbf{B}$, the approved program extends the multihadron program started at Bates. Future plans include studying parity violation and the strangeness content of hadrons at CEBAF and SLAC, and an extension of multihadron studies at the microtron facility at Mainz, Germany. 


\section{MASSACHUSETTS INSTITUTE OF TECHNOLOGY}

Laboratory for Nuclear Science

Cambridge, Massachusetts 02139

Title of Project: Center for Theoretical Physics: Theoretical Nuclear Physics

Person in Charge: J. Negele

Telephone: $\quad$ (617) 253-7077

Scientific Staff: $\quad$ M. Baranger, A. Branchina, M. Burkhardt, J. Cahallero, T. W. Donnclly, H. Feshbach, P. Hoodbhoy, L. Jacobs, X. Ji, A. Kerman, B. Lauritzen, E. Lomon, G. Marbach, C. Martin, R. Martin, E. Moniz, M. Musolf, M. Oleszczuk, T. Otofuji, H. Pirner, J. Polonyi, E. Swanson, D. Wasson

FY 1992 Funding: $\quad \$ 1,155,000$

The goal of our research in theoretical nuclear physics is to understand the structure and interactions of the nucleons, atomic nuclei, and dense matter of which the universe is composed.

One primary focus of our current research is understanding the role of quarks and gluons in nuclear physics. We are currently undertaking large scale numerical calculations to solve QCD to determine the quark structure of the nucleon, as well as exploring new analytical techniques and studying quark models of nucleons and nuclear forces.

Pioneering studies of quark and gluon structure functions in nucleons and nuclei have continued, with particular emphasis on spin-dependent effects. A new class of chirally odd structure functions was discovered which cannot he measured in conventional deep inelastic scattering experiments, and novel polarized hadron experiments have heen proposed to measure them.

The nuclear many-hody theory provides the foundation for many aspects of nuclear theory, and we continue to explore new approaches to many-body physics. Current investigations include study of the role of chass in nuclear physics, exploration of the relationship between classical and quantum chaos, and the development of new semiclassical approximations, path integral technicyues, and stochastic methods.

The study of nuclear structure with electron scattering has been . . Jajor focus, hecause of hoth the unique precision of electromagnetic probes and the commitment to the experimental program at Bates. A salient recent accomplishment has heen the development of the use of polarization and coincidence experiments as new tools for fundamental studies with electrons. 


\section{MASSACHUSETTS INSTITUTE OF TECHNOLOGY}

\section{Laboratory for Nuclear Science}

Cambridge, Massachusetts 02139

Title of Project: Relativistic Heavy-Ion Collisions

Person in Charge: $\quad$ S. Steadman

Telephone: $\quad$ (617) $253-4155$

Scientific Staff: $\quad$ W. Kehoe, C. Ogilvie, L. Rosenberg, J. Ryan, G. Stephans, R. Verdier

FY 1992 Funding: $\$ \$ 775,000$

We have embarked on the study of the interaction of extremely relativistic massive nuclei bombarding targets such as $\mathrm{Au}$. These interactions will produce nuclear matter at the limits of its matter and energy density, as much as an order-of-magnitude greater than that observed in normal nuclei. Under these conditions, one may expect a new phase of nuclear matter to be observed, namely a plasma formed from the quarks that make up the nuclei and the gluons that bind the quarks. Our group is seeking to detect this new phase of nuclear matter, as well as to understand these reactions in this new energy regime.

We are a principal collaborator in Experiments E802/E859/E866 (S. Steadman, cospokesman), at AGS at BNL. This experiment first hegan collecting data using $14.6 \mathrm{GeV} /$ nucleon Si beams in the spring of 1987. In the spring of 1992, we began using $10.7 \mathrm{GeV}$ nucleon Au beams now available using the recently commissioned booster synchrotron. The experiment consists of a rotatable spectrometer that measures the distribution of emitted charged particles, their type, and energies. The spectrometer consists of tracking chambers hefore and after a momentum-analyzing magnetic field, together with a highly segmented time-of-flight wall and segmented Cerenkov counter system. Typically, several particles are tracked simultaneously through the system in a given event.

We have observed large probabilities for strangeness production $\left(\mathrm{K}^{+} / \pi^{+}\right.$ratio) in head-on collisions with Au targets, namely, about 3 (for $\mathrm{Si}$ ) to 5 (for $\mathrm{Au}$ ) times larger than in proton-proton collisions under similar conditions. Such large strangeness production has been an expected consequence of the formation of the quark-gluon plasma.

We are also a major part of the $100 \mathrm{GeV}$ /nucleon RHIC PHOBOS Collahoration (W. Busza, spokesman). This compact experiment will measure charged particles at low transverse momentum near midrapidity, using a series of $\mathrm{Si}$ pad and strip detectors in two pairs of superconducting coils. We also are planning to use this PHOBOS detector at the BNL AGS in 1996 (G.S.F. Stephans, cospokesman). 


\author{
Laboratory for Nuclear Science \\ Cambridge, Massachusetts 02139 \\ Title of Project: $\quad$ Solar Neutrinos \\ Person in Charge: $\quad$ M. Deutsch \\ Telephone: $\quad$ (617) $253-4289$ \\ Scientific Staff: $\quad$ M. Deutsch \\ FY 1992 Funding: $\quad \$ 29,000$
}

Neutrinos from the Sun are potentially a powerful source of information concerning the solar interior. Neutrinos of different energies arise at different stages of the nuclear reaction chain by which the Sun generates energy. These different stages occur in different regions of the interior, depending on the temperature. A good measurement of the flux and energy distribution of solar neutrinos would therefore be a powerful test of our understanding of solar energy production.

Results of four experiments have been reported in the literature. All report substantially lower counting rates than expected from current astrophysical models of the Sun, but the systematic and statistical uncertainties are still quite large. There is a consensus that the deficiency, if confirmed, is probably not due to a low rate of emission from the Sun, but to a new process that makes the neutrinos less readily detectable when they arrive at the Earth. Confirmation of this process would be an important advance in our understanding of elementary particles.

Because of the very weak interaction of neutrinos with matter, experiments must be carried out deep underground to reduce background due to cosmic-rays, and they require large amounts of material exceptionally free of radioactive impurities. Our experience with such impurities, extending over half a century, allows us to make a significant contribution. We are collaborating with groups from Italy, Russia, and the U.S. in an experiment to detect the neutrinos from one particular, critical step in the solar nuclear cycle-the decay of ${ }^{7} \mathrm{Be}$. The experiment is being prepared in the underground laboratory tunnel under the Gran Sasso d'Italia in central Italy. 
Title of Project: $\quad$ Fast Neutron Cross Section Measurements

Person in Charge: $\quad$ G. F. Knoll

Telephone: (313) 936-0121

Scientific Staff: $\quad$ D. Wehe, M. Christodoulou, N. Tsirliganis', J. Yang

FY 1992 Funding: $\quad \$ 210,000$

Our efforts are concentrated on carrying out accurate measurements of cross sections that describe the interactions of fast neutrons in materials of interest in the design of nuclear energy systems. Cross section data of this type are then evaluated and made available to the user community in the form of computer-based data sets. With the aid of such data, the feasibility of various fission and fusion concepts can be explored and predictions made on the performance to be expected from specific designs. Present experimental efforts are centered on the measurement of yields and angular distributions of secondary neutrons that are produced by the interaction of primary neutrons with an energy of $14 \mathrm{MeV}$. Such neutrons are the principal form in which energy is released in the fusion reaction, and a detailed understanding of their interaction probabilities is vital in the investigation of fusion reactors as well as other systems in which the behavior of fast neutrons is important.

We have constructed a unique facility for these measurements, and it is now in routine operation. Neutrons are producted by a beam of $150 \mathrm{keV}$ deuterons incident on a tritium target. In most of our measurements, the beam is pulsed and time compressed with a klystron buncher to produce bursts of 1 nanosecond duration. The corresponding burst of $14 \mathrm{MeV}$ neutrons then strikes a target of the material of interest, and we employ time-of-flight techniques to measure the energy spectrum of the secondary neutrons produced in the target. Flight paths up to 10 meters are accommodated within a large volume, heavily shielded laboratory. Special efforts have been made to keep the mass of materials in the vicinity of the neutron source as small as possible to minimize the influence of neutrons scattered in nearby structures in order to enhance the accuracy of the data. Spectra are recorded using a movable, heavily shielded fast neutron detector and computer-based time-of-flight data acquisition system.

We have completed initial measurements using an iron target, and measurements with targets of bismuth and niobium are in progress. Additional measurements are scheduled using targets of chromium and nickel, and we are evaluating the feasibility of carrying out similar measurements on more than a dozen other elements of potential interest in fusion reactor designs. 
School of Physics and Astronomy

Minneapolis, Minnesota 55455

Title of Project: Pion- and Proton-Nucleus Interactions at Intermediate Energy

Person in Charge: $\quad$ D. Dehnhard

Telephone: $\quad$ (612) 624-4562

Scientific Staft: $\quad$ J. L. Langenbrunner, M. Palarczyk, M. A. Franey

FY 1992 Funding: $\quad \$ 20 \%, 000$

The forces between free nucleons as well as between mesons and free nucleons are modified in the nuclear medium. These modifications need to be understood in order to describe nuclear properties and reactions in terms of the elementary two-body forces. Our goal is to provide the experimental data needed to test our theoretical understanding of these modifications and of the reaction mechanisms and to use the special properties of the pion-nucleon force to elucidate the nature of nuclear excitations not easily accessible to electromagnetic probes.

In experiments with intermediate energy pions at LAMPF, we have found that the cross section ratios using positive and negative pions for transitions to discrete nuclear states are reproduced quite well utilizing free-scattering amplitudes and our current knowledge of these excitations. However, in a recent elastic scattering experiment on a polarized target, we found that either current models of spindependent transitions need to be revised or that medium modifications are quite significant. As well, our measurement of transitions to the unbound nuclear continuum shows large deviations from predictions based on the free force.

At the Indiana University Cyclotron Facility, we recently measured the first neutron spectra from proton bombardment of ${ }^{4} \mathrm{He}$. These spectra are of great interest for current efforts in understanding mass-4 nuclei and for astrophysical theories of solar flares and mass accretion of neutron stars.

In the near future, we plan to measure pion scattering from polarized ${ }^{3} \mathrm{He}$ nuclei. Because few-nucleon theories now provide reliable wavefunctions for ${ }^{3} \mathrm{He}$, this experiment promises the hest possible test so far of the pion-nucleus interaction. Other plans include an extension of the research to higher energy pions and to kaon-nucleus scattering. 


\section{UNIVERSITY OF MINNESOTA}

School of Physics and Astronomy

Minneapolis, Minnesota 55455

Title of Project: $\quad$ Research in Theoretical Nuclear Physics

Persons in Charge: P. J. Ellis, J. I. Kapusta, L. D. McLerran

Telephone: $\quad$ (612) 624-0506

Scientific Staff: $\quad$ M. Carrington, K. Geiger

FY 1992 Funding: $\quad \$ 320,000$

The goal of our research is to understand the properties of matter at energy densities beyond that in atomic nuclei. Such high density matter exists in neutron stars, it existed early in the Big Bang, and it exists briefly during collisions of massive nuclei at accelerators at Brookhaven and CERN. Advances in our understanding of the physical world are made by comparison between theoretical predictions and experimental observations. Our theoretical calculations are based at the fundamental level on forces described by QCD and electroweak theory. We use the tools of relativistic quantum field theory and quantum statistical mechanics.

There are three fundamental phases of matter. At lower energy densities, matter consists of nucleons, pions, and other hadrons. At a temperature of about $160 \mathrm{MeV}$, there is probably a deconfinement/chiral symmetry restoring transition to quarks and gluons. At the much higher temperature of about $100 \mathrm{Gev}$, there is a transition to a phase where the symmetries of electroweak theory are restored. The lower temperature transition is of utmost importance for the ultrarelativistic heavy-ion collisions to occur at RHIC, while the higher temperature transition certainly occurred in the early universe and could have been the point at which the baryon number of the universe was finally determined.

We are working on a wide variety of problems in these areas; they can be categorized roughly as follows: First, one needs to understand the equilibrium properties of the three phases. Then, one needs to understand the order of the phase transitions, their properties, and their dynamics. The nonequilibrium dynamics of heavy-ion collisions is described in terms of a parton cascade which then must be supplemented with the dynamics of the hadronization transition. Experimental observables can be calculated, such as photon, dilepton, and hadron distributions. The baryon changing processes in the early universe occur in the walls of expanding bubbles and are presently being studied. The dynamics of these expanding bubbles is very close to the problem of the hadronization transition to he studied at RHIC. 
School of Physics and Astronomy

Minneapolis, Minnesota 55455

Title of Project: Nuclear Physics Studies at Intermediate Energies

Person in Charge: $\quad$ N. M. Hintz

Tolephone: $\quad$ (612) 624-5871

Scientific Staff: $\quad$ A. Sethi, V. Starodubsky, M. Franey

FY 1992 Funding: $\quad \$ 122,000$

The research program consists of the study of nuclear structure, nuclear reaction mechanisms, and the nature of the interaction between free nucleons (neutrons and protons) and nucleons in nuclear matter. Most of the experimental work is done at LAMPF or the Indiana University Cyclotron Facility (IUCF). The experiments typically involve directing beams of protons at targets containing the nuclei to be studied. Energetic particles that emerge from the target are detected at the focus of a magnetic spectrometer to provide high precision data on nuclear excitation energies. Considerable theoretical work must be done to extract the nuclear information from the primary data. Various assumptions must be made about the mechanisms of the reaction and the nature of the forces involved.

Our current research is focused on testing the consequences of predictions, based on QCD sum rules, of a reduction of nucleon and meson masses in the nuclear medium. Such a reduction of masses modifies the interaction between nucleons because the ranges and strengths of the meson exchange potentials are altered. We are carrying out new theoretical analyses of current and previous experimental data on intermediate energy (135-800 MeV) proton scattering by nuclei using these modified forces. The data so far have been consistent with these ideas. An experiment is underway to study the quasifree scattering of protons by protons in nuclei to measure directly the effective interaction in nuclear matter. In the future, we will explore both relativistic and nonrelativistic reaction models.

Another part of our research program is the experimental and theoretical study of collective excitations in shape transition nuclei to test predictions of the Interacting Boson Model. We have found that the addition of $\mathrm{g}$-bosons $(\mathrm{L}=4)$ to the model is needed for an explanation of the data. 


\section{UNIVERSITY OF MINNESOTA}

School of Physics and Astronomy

Minneapolis, Minnesota 55455

Title of Project: Interaction of Gold and Other Massive Relativistic Nuclei

Persons in Charge: C. J. Waddington, P. S. Freier

Tolephone:

(612) 6i: 566

FY 1992 Funding: $\quad \$ 84,000$

Studies of the interactions between massive nuclei at high energies have become an exciting field of nuclear physics. New accelurator capabilities now allow these interactions to be studied under controlled conditions rather than only in the cusmic radiation. The high energies available in these interactions result in the creation of large numbers of subatomic particles and lead to a study of the behavior of nuclear matter under conditions of extreme temperature and compression. Under these conditions, it is predicted that the individual nucleons will break up into their constituent quarks and gluons to form a new form of matter, the quark-gluon plasma. Proof of the formation of this plasma would confirm current mouels of nuclear matter. A search for evidence of the formation of such a plasma is the main objective of this program. An added objective is to study the modes by which these energetic nuclei breakup into fragments in these interactions.

In collaboration with colleagues at Lowisiana, Krakow, and Moscow, we are currently studying the interactions of $200 \mathrm{Gev} /$ nurkeon sulfur nuclei accelerated at CERN in emulsion targets, concentrating on those interactions in which the nuclei collide head-on. These are the collisions that are most likely to show evidence for the formation of a plasma. By measuring the emission angles of every produced article in each inteiaction, we obtain a global view of the interactions ihat allows us to search for evidence of any deviations froni normal.

Recently, we also exposed emulsion targets to gold nuclei accelerated at Brookhaven with i1.3 Gev/nucleon. The study of the interactions of these very massive nuclei should explore a new region of nuclear behavior and will be compared with previous studies made at energies an order-ofmagnitude lower. It is anticipated that by next year, CERN should be able to provide similar nuclei at energies of $200 \mathrm{Gev} /$ nucleon. 
Department of Physics and Astronomy

Mississippi State, Mississippi 39762

Title of Project: Gammasphere Software Development

Person in Charge: $\quad$ R. B. Piercey

Telephone: $\quad$ (601) $325-2688$

FY 1992 Funding: $\$ \$ 52,000$

When finished, the Gammasphere will he the most ambitious and most powerful detector array ever constructed for studying detailed nuclear structure. The current Gammasphere data acquisition system design calls for a multicrate, VME frontend coupled to a distributed system of UNIX-based workstations. While development of the hardware elements of the system will require substantial effort, the production of the computer software for Gammasphere may he the most difficult task and has been identified by the Gammasphere Steering Committee as lying on the critical path for completion of the Gammasphere project. The Department of Physics and Astronomy at Mississippi State University is addressing this crucial issue by developing new software techniques and applications for use at the Gammasphere facility.

As the Chairman of the Gammasphere Software Working Group (SWG), we are organizing regular meetings to encourage participation and information exchange among the major nuclear physics laboratories and university research groups. Input from the user community is passed on to the Gammasphere facility director, and specific areas are assigned for further study and research. Current areas being studied by SWG include: data acquisition models and metaphors, graphical user interfaces for the Gammasphere, standard data objects (histograms, multiparameter data, elc.), parallel processing techniques, and analysis of high fold spectra.

In addition to our work with SWG, we are developing a graphical user interface for the histogramming software provideci hy HHIRF. Other work includes the development and prototyping of a universal histogram object (UHO) to provide a level of standardization for histogram data structures. Such a standard, if widely accepted, would help reduce the tedious translation effort required when data are shared among institutions using very different analysis software.

Future work will include the refinement and testing of UHO and the development of consistent standards for multiparameter data. A well documented, general format for histogram und multiparameter data will simplify the sharing of data products from nuclear experiments and result in reduced manpower and computing costs. 


\section{UNIVERSITY OF NEW HAMPSHIRE}

Department of Physics

Durham, New Hampshire 03824

Title of Project: $\quad$ Electromagnetic Studies of Nuclear Structure and Reactions

Persons in charge: J. H. Heisenherg, J. R. Calarco

Telephone: $\quad(603) ; 862-1951$

Scientific Staff: $\quad$ J. F. Dawson, J. Distelbrink, F. W. Hersman, T. Smith

FY 1992 Funding: $\quad \$ 482,000$

Our research program seeks a more complete understanding of the structures and forces of nuclei using reaction experiments and nuclear modeling. We conduct our experiments at electron accelerators to exploit the precision of the electron probe. Our nuclear modeling efforts provide interpretation and guidance by exhibiting the dependence of the reaction observables on the physical description of the nucleus.

We recently finished studies of the low lying discrete states of lead and cerium, as well as a comparison of nuclei in the zirconium region. We separated the various contributions to the responses of the nuclear giant resonances of carbon and oxygen using coincidence measurements performed at Mainz, and more recently, Bates. We are widely recognized for our contributions to nuclear structure in the low lying discrete states and giant resonance regions.

We completed a measurement of proton knockout from oxygen at National Institute for Nuclear Physics and High Energy Physics, Amsterdam (NIKHEF) to determine the proportion of protons that occupies orbitals with higher energy than the ones that are normally occupied. This will help identify the excitation mechanism. More recently, we initiated a measurement of electrons knocking two protons out of the nucleus to further identify these excitation mechanisms. We recently modeled each of these processes to determine the relationship between the measured reaction and the properties of the interaction between nucleons inside the nucleus. Our emphasis is increasingly evolving toward direct coincidence reactions like these.

Our plans for the near future center on preparations for the new continuous beam electron accelerators. We are fabricating a major portion of the large acceptance detector system at CEBAF to extend our studies to higher energies. We have proposed a new detector system for Bates and plan coincidence reactions there with polarized targets. We have approval to pursue studies of the excited states of the proton in Novosibirsk, Russia. 
Department of Physics and Astronomy

Albuquerque, New Mexico 87131

Title of Project: Neutrino Physics

Persons in Charge: B. Dieterle, C. Leavitt

Telephone: (505) 277-2616

Sciontific Staff: R. Reeder

FY 1992 Funding: $\$ \$ 193,000$

We are continuing our focus on the properties of neutrinos through the process of double beta-decay experiment (now completed) and neutrino oscillations and interactions at medium energy.

The experiment on the double beta-decay of ${ }^{100} \mathrm{Mo}$ has been carried out using an enlarged solid state detector array located 4,000 feet underground to eliminate cosmic-ray background. Data-taking is complete, and preliminary results indicate a half-life limit in the range of $10^{22}$ to $10^{23}$ years, which is a significant result.

Our main effort is directed at neutrino oscillations and interactions through collaboration in a new approved experiment at LAMPF, E1173. This experiment uses a relatively large (200 ton) combined Cerenkov and scintillation counter of novel design, which combines the proven technique of Cerenkov light cone reconstruction with the capacity of the scintillator to detect slow particles and to assist in particle identification.

The source of neutrinos in this experiment is the decay of mesons produced in a stopping target in the $800-\mathrm{MeV}$ proton beam at LAMPF. A number of experiments can be carried out, including neutrino and antineutrino oscillations with unprecedented precision, neutrino-electron and neutrino-proton elastic scattering, scattering from carbon, and rare meson decays involving neutrinos.

A critical factor in the design of this detector is the type and concentration of scintillator to be added to the Cerenkov medium (mineral oil). We have been carrying out extensive tests to ensure that light cone reconstruction will be unimpeded, while maximum information can be obtained from the scintillator light output. In addition, we have to insure that the mixture will have long term chemical stahility.

In the future, we will be participating in the construction of the detector, as well as the creation of computer software for the reconstruction of events including both the light cone reconstruction and particle identification. In addition, as the experiment comes on-line, we will contribute to the running of the experiment. 


\section{UNIVERSITY OF NEW MEXICO}

Department of Physics and Astronomy

Albuquerque, New Mexico 87131

Title of Project: Strange Particles and Heavy-Ion Physics

Persons in Charge: D. M. Wolfe, B. Bassalleck

Telephone:

(505) $277-2616$

Scientific Staff: J. Hall

FY 1992 Funding: $\quad \$ 240,000$

We continue to work almost entirely at BNL. We have completed and published the complete results from experiment E811, $\Lambda \rightarrow n+\gamma$. We have collected a sample of events with statistics 75 times greater than the previous world total. Our branching ratio for this decay to all $\Lambda$ decays is $(1.74 \pm 0.16) \times 10^{-3}$. No existing model provides a completely satisfactory description of all the available data on weak radiative hyperon decays. Experiment E814, now complete, was a fixed-target relativistic heavy-ion experiment at $\mathrm{BNL}$. It used $14 \mathrm{Gev} /$ nucleon ${ }^{28} \mathrm{Si}$ beams incident on several different nuclear targets. Many different aspects of the collisions were measured, including transverse energy production, the charged-particle multiplicity production, projectile dissociation, nuclear stopping, low $\mathrm{p}_{\mathrm{t}}$ forward baryons, antiproton and antineutron production, and strangelet production. Strangelets are types of matter with unusually large numbers of strange quarks. The experimental collaboration has published numerous results, among those are that the transverse energy spectra exhibit a thermal shape with rapidity and centrality dependent on the temperature, and evidence for full stopping in very central collisions. It has also demonstrated rescattering effects in heavier nuclei such as $\mathrm{Pb}$. This experiment is being continued with the new Au beams available at AGS. While we are leaving our equipment with this new experiment, E877, none of our personnel will be participating. Additional heavy-ion searches for strangelets are being performed in experiment E886, in which we are active participants. The apparatus and even the beam line are quite different from E814 or E877.

We continue to work on E813, a search for the H-particle. This is a strangeness-2 dibaryon, supposedly bound against strong decay. It may also be regarded as the lightest example of a strangelet. This experiment represented the bulk of our effort during 1992, during which time approximately 40 percent of our desired $10^{12}$ incident kaons were made available. Analysis is being done at the University of New Mexico and Carnegie-Mellon.

Finally, our long term plans include experiment E865, a search for the rare kaon decay $\mathrm{K}^{+} \rightarrow \pi^{+}+\mu^{+}+\mathrm{e}^{*}$. Planning and construction of apparatus, including the data acquisition system, is underway. 
Title of Project: $\quad$ Experimental Studies of Pion-Nucleus Interactions and Nucleon Structure Functions

Person in Charge: G. R. Burleson

Telephone: (505) 646-3831

Scientific Staff: $\quad$ G. S. Kyle, B. K. Park, M. Wang

FY 1992 Funding: $\$ 253,000$

We are studying the interaction of pions with nucleons in nuclei to understand how this interaction is modified by the other nucleons in the nucleus and to improve our understanding of nuclear structure. In particular, we are investigating how pions undergo multiple scattering and absorption in nuclei, what role nucleon-nucleon correlations play in these processes, and what pions can tell us about the spin structure of nuclei. We are also preparing to study the internal spin structure of nucleons, as related to the quarks within them.

Our current research program involves the use of several new or modified systems to which we have made important contributions. We are using the Large Acceptance Detector System at the Paul Scherrer Institute (PSI) to study multiparticle final states resulting from pion absorption in nuclei. The modification of the high energy pion channel (P-Cubed) and the Large Aperture Spectrometer at LAMPF for high resolution work has allowed us to investigate pion elastic and double-chargeexchange (DCX) scattering at energies up to $500 \mathrm{MeV}$. With polarized nuclear targets, we are studying the spin dependence of elastic, charge exchange, and quasi-elastic pion scattering, both at LAMPF and PSI. The DCX results have generally disagreed with most predictions, indicating the importance of other effects hesides simple multiple scattering. The data from polarized targets found an unexpected quenching of spin-flip scattering in certain nuclei, a phenomenon we plan to study further with polarized ${ }^{3} \mathrm{He}$, a nucleus whose structure is fairly well known.

Other future plans include measurement of low energy pion scattering from polarized protons, to investigate the fundamental physics associated with chiral-symmetry breaking and charge-symmetry breaking, and experiments with the new Neutral Meson Spectrometer at LAMPF, which will allow for the first high resolution studies with neutral pions. We also plan to work with the Hermes project at Hadron Elektron Ring Anlage on measurements of the deep inelastic scattering of polarized electrons on polarized nucleons, to study the internal spin structure of the nucleon. 
Department of Physics

Las Cruces, New Mexico 88003

Title of Project: The Theory of Hadronic Systems

Person in Charge: $\quad$ W. R. Gibbs

Telephone: $\quad$ (505) 646-6711

FY 1992 Funding: $\quad \$ 36,000$

While recent progress in high energy physics has led to a candidate for the fundamental theory of the strong interaction, QCD, little benefit has accrued to our understanding of hadronic physics on the nuclear level. In order to make use of this recent advance, we make models of QCD and connect them either directly with nuclear physics observables or with existing theories (primarily models in which the force is described by the exchange of mesons). It is a major objective of this project to study these connections.

To this end, it is necessary to confront the theories involving mesons and those dealing directly with quarks and gluons (the fundamental entities of QCD) with data. Restricting the studies to interactions among neutrons and protons would lead to an inadaquate data set. Because these theories deal with all particles in the hadronic spectrum, the comparison of predictions within many such systems can be made. R.ecent work has involved pi-mesons, K-mesons, and antiprotons interacting with neutrons and protons.

These studies lead to direct results for the nuclear systems. As an example, we consider the case of the failure of "isospin symmetry." Although the neutron-neutron and proton-proton forces are nearly identical (after the electromagnetic interactions have been removed), there appears to remain a small difference. To cite one of several examples, the binding energy of ${ }^{3} \mathrm{He}$ and ${ }^{3} \mathrm{H}$ differs by an amount larger than can be explained hy the electrostatic replusion between the two protons in ${ }^{3} \mathrm{He}$. This smaller binding energy is generally attributed to "mixing" among the mesons being exchanged. This same theory of mixing has been applied to the scattering of pi-mesons from neutrons and protons and has been tested in a different environment.

Future work will continue this direction with the comparison of QCD and mesonic degrees of freedom in the description of nuclear phenomena. 
Title of Project: Study of Relativistic Heavy-Ion Collisions

Person in Charge: $\quad$ M. A. Kramer

Telephone: $\quad$ (212) 650-5576

Scientific Staff: $\quad$ S. J. Lindenbaum

FY 1992 Funding: $\$ 48,000$

The generally accepted theory of nuclear and elementary particle physics is QCD. This project investigates the interactions between quarks by studying relativistic heavy-ion collisions. We use a TPC in a large magnetic field to track and momentum analyze the large number of particles that is produced.

In QCD, the neutrons and protons (nucleons) of "ordinary" matter are each made up of three quarks bound very strongly together by the strong (or nuclear) force. Nuclei of normal matter can be considered to be made of such nucleons. Studying the underlying force is difficult because the theory predicts that "free" quarks will never be seen. In heavy-ion collisions, many secondary particles may be produced, and high values of particle and energy density can be reached. Theory and calculations predict that at sufficiently high particle/energy densities, matter will undergo a phase change; namely, the individual nucleons will "dissolve" and a region of quark-gluon plasma in which the individual quarks will no longer be bound into individual nucleons will be formed. Unfortunately, the theory also predicts that these individual quarks cannot exist once they leave the region of the plasma. Thus, the experiment needs to be able to examine the products of the collision for evidence of such a transition having occurred.

Results have been published on the yield of pions and strange particles with incident silicon ions. The large solid angle accepted allows observations of neutral vee's and particle production over a broad range. Analysis is proceeding on further data taken with incident silicon beams on various targets. A proposal is being developed for the new gold beams. The higher $\mathrm{Z}$ will produce more particle/energy density and make the quark-gluon plasma transition more likely. 
Department of Physics and Astronomy

Buffalo, New York 14260

Title of Project: Study of Relativistic Heavy Ions

Person in Charge: $\quad$ P. L. Jain

Telephone: (716) 636-2538

Scientific Staff: $\quad$ G. Singh, A. Mukopadhyay

FY 1992 Funding: $\quad \$ 100,000$

The goal is to produce and to detect in a baryon-rich environment a new plasma formed from the quarks that make up the nuclei and the gluons that bind the quarks. In our experiment EMU08 at CERN Super Proton Synchrotron (SPS) with ${ }^{16} \mathrm{O}$ and ${ }^{32} \mathrm{~S}$ at $200 \mathrm{AGeV},{ }^{16} \mathrm{O}$ at $60 \mathrm{AGeV}$ and experiment 847 at $\mathrm{BNL}$ with ${ }^{28} \mathrm{Si}$ at $14.5 \mathrm{AGeV}$ in nuclear emulsions, we find energy density $\sim 3$ $4 \mathrm{GeV} / \mathrm{fm}^{3}$ for highest multiplicity events in ${ }^{32} \mathrm{~S}$ and ${ }^{16} \mathrm{O}$ at $200 \mathrm{GeV}$. Multiplicity and angular distributions of shower particles produced in these collisions fit successfully to the predictions of theoretical multistring model VENUS. For central collisions events, an intermittency power law behavior is observed in all beams.

We have been able to produce, detect, analyze, and compare the interactions of other secondary beams, produced in peripheral interactions from ${ }^{32} \mathrm{~S}$ and ${ }^{28} \mathrm{Si}$ projectile at almost the same primary energy as the parent beam, by making use of the unique features of emulsion techniques that serve as a continuous target and detector throughout their entire volume.

For very large impact parameter collisions, events associated with electromagnetic dissociation (ED) were observed where a projectile is excited by the Coulomb field of the target nucleous through the photon exchange into a giant resonance. Experiments at CERN and BNL have shown the increase in ED with energy. For ${ }^{238} \mathrm{U}$ and ${ }^{84} \mathrm{Kr}$ at $\sim 1 \mathrm{AGeV}$ from the Bevalac (LBL), we observed highly excited nuclear composite systems that decay via the emission of intermediate mass fragments, which also give evidence of an intermittency pattern of fluctuations.

We have recently performed an Experiment No: 875 at BNL with massive projectile $\mathrm{Au}$ beam( $-12 \mathrm{AGeV})$, and in 1993-94, we shall use $\mathrm{Pb}$ beam from CERN's SPS (Experiment No: EMU11) and shall try to understand these reactions in the new energy region and shall compare these results with the present data. 
Department of Chemistry

Stony Brook, New York 11794

Title of Project: Nuclear Reaction Studies

Person in Charge: J. M. Alexander

Telephone: (516) 632-7904

Scientific Staff: $\quad$ N. N. Ajitanand, M. T. Magda

FY 1992 Funding: $\quad \$ 230,000$

Our objective is to study the evolution of nuclear properties with increasingly larger amounts of energy and rotational motion. We are trying to help bridge the gap between two energy domains:

(1) the region of temperatures $\leq 6 \mathrm{MeV}$ where composite nuclei are held together by their mean field, and (2) the high energy domain where unbound nuclear clusters may briefly exchange energy before shattering into many particles or fragments. We seek a picture of the variation of nuclear sizes and shapes with temperature and spin. It is in the continuum spectra of particles, fragments, and photons that one must search out the properties of nuclei with temperatures over $1 \mathrm{MeV}$. In reactions between complex nuclei, there is a very brief period $\left(\sim 10^{-22} \mathrm{~s}\right)$ for energy mixing among the nucleons (i.e., thermalization). Then, there is a rather long period $\left(\sim 10^{-21} \mathrm{~s}\right)$ of thermal equilibrium with associated particle evaporation and fission-like decay. Light charged particles have often been observed from both the pre-equilibrium and evaporation-like phases. The framework of statistical mechanics provides a means of using charged-particle emission as a probe of shapes and/or sizes of these hot nuclei $(\mathrm{T} \leq 6 \mathrm{MeV}$ ). Our research program includes both experimental and theoretical efforts in this direction. We have extended the theory to include the role of deformation and have applied the model to observations of particle emission from many reactions. A crucial property of hot nuclei is their lifetime. We have used small angle correlations between ${ }^{2} \mathrm{H}-{ }^{2} \mathrm{H}$ pairs to estimate such decay lifetimes $\left(0.5-1 \times 10^{-21} \mathrm{~s}\right)$. This technique provides a means of tracking the lifetime from slow evaporative decay to faster pre-equilibrium emission. We are using these techniques to compare moderately hot nuclei $(\mathrm{T} \sim 3 \mathrm{MeV})$ to hoth cold nuclei $(\mathrm{T} \sim 0)$ and very hot nuclei $(\mathrm{T}>5 \mathrm{MeV})$. Detailed experiments are being used to try to separate the effects of thermal and rotational motions. 


\section{STATE UNIVERSITY OF NEW YORK AT STONYBROOK}

Department of Physics

Stony Brook, New York 11794

Title of Project: $\quad$ Research in Theoretical Nuclear Physics

Persons in Charge: G. E. Brown, A. D. Jackson, T. T. S. Kuo

Telephone:

(516) $632-8135$

Scientific Staff: $\quad$ C. Adaini, V. Koch, M. Prakash, T. Schaefer, E. Shuryak, J. Verbaarschot, L. Xiong, I. Zahed

FY 1992 Funding: $\quad \$ 793,000$

The Stony Brook nuclear theory program is concentrated in four areas: (1) strong interaction physics, including the physics of hadrons and the consequences of QCD in nuclear systems at finite temperature and high density; (2) nuclear astrophysics; (3) nuclear structure and many-body theory; and (4) nuclear tests of fundamental interactions.

In our studies in strong-interaction physics, we have shown that the masses of vector and scalar mesons go smoothly towards zero at high densities and temperatures. With density, the decrease in mass is sufficiently rapid so that many consequences are observed in nuclear spectroscopy and scattering already in nuclei. The decrease in mass with temperature has the consequence that there is no phase transition from hadrons to quarks and gluons. Indeed, there remains an interesting hadron spectroscopy at high temperatures, which we are working out.

We have shown that a strangeness condensation phase transition occurs in the dense matter reached in the collapse of large stars. This considerably decreases the pressure at high densities and implies that neutron stars are stable only up to a limit, which we estimate to be $1 \frac{1}{2}$ times the mass of our Sun.

Given this, there is a strong possibility that the core of the last nearby supernova, SN1987A, went into a black hole.

Through the use of mean fields, we are investigating spectra of particles emerging from the Brookhaven relativistic heavy-ion experiments. From earlier analyses of experiments with light projectiles, we are predicting results of the gold on gold experiments.

We continue to work out effective interactions to use in nuclei, beginning from the elementary nucleon-nucleon experiment. These effective interactions are being employed in heavy nuclei to explain elementary processes such as double beta decay. 
Department of Earth and Space Sciences

Stony Brook, New York 11794

Title of Project: Research in Nuclear Astrophysics: Stellar Collapse and Supernovae

Persons in Charge: J. M. Lattimer, A. Yahil

Telephone: $\quad$ (516) 632-8227

FY 1992 Funding: $\quad \$ 101,000$

The fundamental objective is to relate nuclear physics to astrophysics by concentrating on the problem of the gravitational collapse of massive stars, thought to lead to Type II supernovae and neutron star formation. This entails development work for (1) the equation of state of hot, dense matter, (2) weak interaction processes in dense matter, and (3) neutrino radiation hydrodynamics. By focusing on the observables of Type II supernovae, chiefly the luminosities and spectra of the emitted neutrinos, dense matter physics could be constrained when simulations are compared to observations of supernovac in our galaxy.

Work on the equation of state focuses on the microscopic compressible liquid drop model for nuclei immersed in a nucleon gas. This equation of state is the first astrophysically useful one that contains adjustable parameters and includes important correlations among parameters, like the specific heats, incompressibility, and symmetry coefficients. This is necessary hecause some parameters have not heen accurately determined hy experiment. The inclusion of these correlations is essential for a correct understanding of the gravitational collapse stage of supernovae. (Similar arguments may apply to the interpretation of heavy-ion collisions.)

Numerical simulations of supernovae using radiation hydrodynamics codes are being carried out. A conventional code is used to investigate the early supernova stages; however, such codes are limited to very small timesteps, and their accuracy in modeling later stages of the explosion, after millions of timesteps, is questionable. This problem is surmounted with an "implicit" code, which will be used to investigate the later explosion stages and whether or not neutrino heating is the explosion mechanism.

Observations of the first few seconds of neutrino emissions from supernovae will shed much light on the explosion mechanism and, possibly, on aspects of physics currently unobtainable in conventional nuclear experiments (e.g., the supra-nuclear symmetry energy and possible phase transitions to meson condensates and/or quark matter). The long-term evolution of neutron stars is also modeled. We have discovered neutrino cooling mechanisms (direct nucleon and hyperon Urca processes) that may cool neutron stars more efficiently than previously believed. Observations of thermal emissions ( $x$ rays) from neutron star surfaces by satellites might elucidate properties of supra-nuclear matter. 
Department of Chemistry and Physics

Norfolk, Virginia 23504

Title of Project: Polarization Experiments with Hadronic and Electromagnetic Probes

Person in charge: V. Punjabi

Telephone: (804) 683-2472

FY 1992 Funding: $\quad \$ 62,000$

Spin physics will be an important part of the research program at CEBAF. A polarized electron beam will be available soon after turn-on, and experiments using this capability have already been approved by the program advisory committee (PAC) 4 and 5. One such experiment is the measurement of the electric form factor of the proton, which is still not precisely measured, and is of a fundamental nature. Its precise characterization is a prerequisite for the interpretation of numerous nuclear physics experiments that will study the effect of the nuclear medium on the elementary properties of the constituent nucleons.

In Hall $\mathrm{A}$ at CEBAF, the hadron high resolution spectrometer will be equipped with a focal plane proton polarimeter (FPP) to measure polarization transfer; this device is to be huilt by outside users. Work in relation with this project will be distributed over several local universities, and Norfolk State is a participant.

Concurrently, a series of experiments to study spin observables in hadron-induced nuclear reaction is being pursued with the synchrotron SATURNE. These experiments involve tensor analyzing power and polarization transfer in the breakup and backward elastic dp interaction, and tensor analyzing power in the breakup of $4.5 \mathrm{GeV}{ }^{6} \mathrm{Li}$. In relation to these measurements, several calibration runs have heen made with the polarimeter POMME, which is of design similar to the planned CEBAF FPP. These measurements have recently been pushed up to $2.4-\mathrm{GeV}$ proton energy, and the preliminary results clearly demonstrate that the polarization transfer experiments proposed at CEBAF will be feasible. 


\section{UNIVERSITY OF NORTH CAROLINA}

Department of Physics and Astronomy

Chapel Hill, North Carolina 27599

Work performed at Triangle Universities Nuclear Laboratory (TUNL)

Title of Project: $\quad$ Studies of Nuclear Processes

Person in Charge: $\quad$ E. J. Ludwig

Telephone: $\quad$ (919) $962-5001$

Scientific Staff: $\quad$ Z. Ayer, A. E. Champagnc, T. B. Clegg, R. K. Das, H. J. Karwowski, W. J. Thompson

FY 1992 Funding: $\$ 350,(0) 0$

Our research emphasizes understanding how forces within nuclei depend on nuclear spins. For this, we mainly use spin-polarized heams of protons and deuterons interacting with various target nuclei. Most of our research is done at Triangle Universities Nuclear Laboratory (TUNL) using the 1()-MV tandem Van de Graaff accelerator and the Low Energy Beam Facility.

Current work includes experimental and theoretical research on tensor forces, which make the lightest nuclei slightly nonspherical and which can he revealed hy measuring how their interactions depend on spin orientation. As part of this research, we study how scattering depends on the structure of the colliding and resulting nuclei, specifically for tritons and helium nuclei ejected in deuteron collisions with nuclei. Other research projects involve the study of the fusion of two deuterons and the scattering of protons from deuterons in order to determine the spin dependence of reactions between the simplest of nuclear systems.

Measurements of the rates of nuclear reactions can provide us with information from which we can learn about the early history of the universe, the evolution of our galaxy, and the structure and evolution of stars. We are developing equipment at TUNL and are using external facilities (at ORNL and at Yale University) to carry out this work. We have recently completed several projects that are relevant to the Big Bang and stellar explosions and are heginning measurements with our new facilities at TUNL.

Recent progress includes completion of and improvements to an atomic heam polarized ion source for TUNL, to produce very intense beams of polarized protons and deuterons needed for many experiments. We have also developed a facility to measure the spin dependence of reactions hetween very light nuclei at the low energies relevant to stellar nucleosynthesis and to nuclear fusion reactors.

Most of our work is part of Ph.D. research projects, with seven University of North Carolina graduate students currently in the research group. Thus, we are contributing to national talent in science and technology. 
Department of Physics

Raleigh, North Carolina 27695

Title of Project: Theoretical Nuclear Reaction and Structure Studies Using Hyperons and Photons

Person in Charge: $\quad$ S. R. Cotanch

Telephone: $\quad$ (919) 515-3316

Scientific Staff: $\quad$ A. Szczepaniak

FY 1992 Funding: $\quad \$ 82,000$

This theoretical research program investigates electromagnetic processes involving the quark substructure of elementary particles as well as the composite nuclear structure of systems of particles. This project emphasizes the electron and photon, for which the basic interaction is understood, as probes of particles containing the strange quark (hyperons) and nuclei composed of one or more hyperons (hypernuclei) and/or excited nucleons (a neutron or proton) called delta particles. The philosophy is to utilize the unique interaction features of these novel systems to perform a consistent, comprehensive theoretical andysis entailing large scale (supercomputer-based) scientific computation. The long-term goal is to desuibe, and therefore understand, data from existing and new accelerators by generating realistic, reliable calculations from a formal framework based on fundamental first principals and exact symmetries of nature.

The current program is a collaborative effort addressing three different but related areas:

(1) relativistic quark models for mesons (pion, kaon, rho, $a_{1}, D$ ) using light-cone dynamics and connected to QCD; (2) chiral Lagrangian covariant analysis of elementary kaon electromagnetic production from proton targets forming lambda, sigma, and excited lambda particles; (3) large scale continuum shell model calculations of medium energy photonuclear nucleon knockout reactions for carbon and oxygen targets. Recent key findings are (1) for all three projects, it is very important to properly incorporate fundamental symmetries especially gauge invariance and for relativistic theories Poincare and crossing symmetry and to comprehensively test the models by computing and analyzing all observables; (2) a simple one-parameter quark model can provide a reasonable description of the static and dynamic properties for the previously mentioned mesons including the pion form factor at high momentum; (3) the limited world low energy kaon photo and electroproduction data for lambda, sigma, and excited lambda (1405) formation and branchiing ratio for saon radiative capture can be described within a unified chiral Lagrangian having phenomenological coupling constants; and (4) using a realistic affective interaction and a large shell model space, including delta isobar excitation, medium energy proton and neutron knockout data can be simultaneously described.

Future work will focus on theory connected to new data anticipated from CEBAF, KEK in Japan, and Uppsala in Sweden. More extensive QCD calculations will be performed for mesons including amplitudes for electromagnetic processes. Explicit gluon degrees of freedom will he included to 
investigate the formation of glueballs and hyhrid mesons having one or more gluons. The medium energy photonuclear project will examine the sensitivity to the short range component of the nucleon interaction and possible nuclear quark degrees of freedom. 
Department of Physics

Raleigh, North Carolina 27695

Title of Project: $\quad$ QCD Quark Model Study of Hadron and Few Hadron Systems

Person in Charge: $\quad$ C.-R. Ji

Telephone: $\quad$ (919) $515-3478$

Scientific Staff: $\quad$ A. Szczepaniak

FY 1992 Funding: $\$ 47,000$

One of the most exciting challenges in nuclear physics is to understand the medium energy internal structure of hadron (e.g., meson and nucleon) and few hadron systems (e.g., deuteron) using the underlying theory of strong interactions, QCD. There are two major reasons for this. First, while the high energy experimental data indicate that hadrons are made of even smaller constituents (i.e., quarks and gluons), the isolated quarks and/or gluons have never been observed experimentally. This implies that there must be an intrinsic confining mechanism of quarks and gluons in QCD. Second, it is not well understood (thus, debated in some cases) whether the medium energy experimental data can be described microscopically by the reactions of quarks and gluons. The key to understand the medium energy internal structure of hadron and few hadron systems is the systematic development of the microscopic quark/gluon model based on QCD and the detailed analysis of precision medium energy experimental data to test the model. This is the major objective of this program.

Research is currently focused on the role that the special relativity plaj's in the motion of quarks and gluons inside the hadron and, specifically, on the mesons in which the constituent quarks can move with the speed close to that of light. Experimental data on the form factors, charge radii, decay constants, quark distribution amplitudes, and mass spectra of pion, kaon, D-meson, r-meson, and axial-vector-meson are compared with the theoretical calculations from the relativistic quark model.

A recent development is the formulation of a simple relativistic quark model based on the idea that the number of cunstituents and the relativistic spin structure of the hadron are well defined on the light-cone of the space and time coordinates. The theoretical predictions on the static properties of mesons are in a good agreement with the available experimental data and the results from the other nonperturbative QCD models such as the QCD sum-rule technique and the lattice QCD simulation. This model is now being further tested and refined to make a direct connection to QCD.

Future work will focus on the precision medium energy measurements at CEBAF. The electromagnetic form factors of pion, kaon, and neutron to be measurepd at CEBAF will generate unambiguous and useful information on the structure of hadrons and reaction mechanisms. 


\section{NORTH CAROLINASTATE UNIVERSITY}

Physics Department

Raleigh, North Carolina 27695

Work Performed at the Triangle Universities Nuclear Laboratory (TUNL)

Title of Project: Studies of Nuclear Structure Using Neutrons and Light Ions

Person in Charge: $\quad$ G. E. Mitchell

Telephone: $\quad$ (919) $515-2521$

Scientific Staff: $\quad$ C. R. Gould, D. G. Haase, E. F. Moore, D. R. Tilley

FY 1992 Funding: $\quad \$ 280,000$

Investigations of Fundamental Symmetries. A major goal of nuclear physics is to study how the symmetries of space and time affect the forces that act between the nucleons in nuclei. At Los Alamos, our work is with the TRIPLE collaboration, and we study parity violation in epithermal neutron resonances in heavy nuclei. Our results in thorium showed that parity violation is a universal resonance phenomenon-all p1/2 resonances showed statistically significant effects. Remarkably, the signs of the effects were all positive-contradicting the expected random distribution in sign. Confirming this correlation in other nuclei and mapping out the mass dependence of the parityviolating signal is the goal of our continuing measurements at Los Alamos. At the Triangle Universities Nuclear Laboratory (TUNL), we have carried out the first measurement of a bound on $\mathrm{T}$-violation in polarized neutron transmission. We used a cryogenically aligned holmium single crystal target to set a bound of 0.1 percent on the T-odd coupling constant in the effective nucleon-nucleon interaction.

Statistical Properties of Nuclear Energy Levels and Tests of Chaos in the Nucleus. The ultrahigh heam energy resolution on the single-ended TUNL accelerator provides unique data. The fluctuation properties of states in ${ }^{26} \mathrm{Al}$ are consistent with random matrix theory from the ground state to the resonance region. These data provided the first experimental test of how a broken symmetry (in this case isospin) affects the fluctuation properties. A very small amount of symmetry breaking seems to restore the fluctuation properties to their form in the complete absence of the symmetry. The present goal is to establish a pure and complete level scheme for ${ }^{30} \mathrm{P}$ to verify this important result.

Nuclear Data Evaluation. 'TUNL is responsible for data evaluation for the nuclei in the mass region $A=3-20$ in accordance with the recommendation to DOE by the Panel on Basic Nuclear Data Compilations of the National Research Council. This project is carried on by H.R. Weller of Duke and D.R. Tilley of North Carolina State University.

Radiative Capture Reactions. The main emphasis of this project is the study of few-nucleon systems utilizing radiative capture experiments with polarized beams as high as $20 \mathrm{MeV}$ extending down to the astrophysical (keV) region. 
Department of Physics and Astronomy

Evanston, Illinois 60208

Title of Project: Strong Interaction Studies with Medium Energy Probes

Person in Charge: K. K. Seth

Telephone: (708) 491-3123

Scientific Staff: $\quad$ D. Dimitriyonnis, M. Sarmiento, R. Soundranayagam

FY 1992 Funding: $\$ 263,000$

This program of experimental research has one central objective: to further the understanding of the strong force that binds quarks and gluons to make the so-called elementary particles, the mesons (such as pions and kaons), and the baryons (such as nucleons, deltas, and hyperons), and is also responsible for the binding of nucleons into nuclei. In pursuit of this goal, we conduct a broadly diversified program of experiments with heams of protons, antiprotons, pions, and electrons at several national laboratories (LAMPF, BATES, NSCAL, and Fermilab) and international laboratories [CERN, National Institute for Nuclear Physics and High Energy Physics-Amsterdam (NIKHEF), Saclay]. The research program has two broadly defined components.

Using the antiproton beams available at Fermilab (2-9 GeV) and CERN (0-2 GeV), we study the properties of light and heavy mesons (qā, $q=$ up, down, strange, or charmed quarks). Our continuing series of experiments at Fermilab (E760) has achieved unprecedented'energy resolution ( 1 part in $\sim 10^{4}$ ) and has provided new insights into the mesons which make the Charmonium family (ci). They have enabled us to investigate the structure of the proton in an entirely new domain (for time-like momentum transfers), and to study the proton-antiproton interaction with very high precision. The experiments are also intended to search for exotic constructs of quarks and gluons, which are predicted to exist but which have not yet been found. In the near future, it is also intended to study the interaction of Charmonium with nuclei.

In our experiments with pion and electron beams, we are currently addressing questions relating to (1) the role of short range nuslear correlations in fion double-charge-exchange reactions, (2) the possible existence of very exotic light nuclei, such as ${ }^{6} \mathrm{H}$ and ${ }^{10} \mathrm{He}$ (earlier we discovered ${ }^{9} \mathrm{He}$ ), (3) identification of the elssive, deeply bound states of the heavy pionic atoms, and (4) the possible change in the properties of the nucleons when they are embedded in the nuclear medium, as manifested in the quar,i-free scattering of electrons. 
Title of Project: Theoretical Studies of Nuclear Structure

Person in Charge: $\quad$ E. R. Marshalek

Telephone: $\quad$ (219) 239-6238

FY 1992 Funding: $\quad \$ 52,000$

At the present stage of progress, our conceptual understanding of nuclear structure is based on a number of phenomenological nuclear models. The more important models can be based more or less successfully on the microscopic shell model of interacting neutrons and protons. One of the aims of the project is to provide a unified conceptual and mathematical foundation for the diversity of models that have been used to describe collective phenomena in atomic nuclei starting from the microscopic shell model. This involves the development of new mathematical tools as well as new models to explain the wealth of accumulating experimental data.

The unifying tool developed by the current project and its predecessors is the mean-field approximation augmented by what is called the boson mapping method, which describes the tendency of nucleons to form collective pairs. This tool is now being applied to understand properties of rapidly rotating atomic nuclei.

One of the recent discoveries is that these nuclei need not always rotate about a principal axis (like a rigid body) but, under certain circumstances, can rotate instead about an oblique axis. Current and future work is centered on delineating the boundaries of this mode of collective motion and on finding experimentally identifiable signatures.

Another recent discovery is that the same tool used to treat collective rotation (cranked mean-field approximation) can also be applied to describe certain types of nonlinear collective oscillations. This approach has just been successfully tested on simple models. In the near future, it will be applied to describe experimentally observed harmonics (multiphonon excitations) of certain collective modes. Of particular interest are the doubly excited gamma vibrations, which are modes occuring in nuclei with permanently deformed shapes. 
Title of Project: Study of Structure of Nuclei with Neutrons and Nuclear Data Measurements for Magnetic Fusion Energy

Persons in Charge: $\quad$ R. O. Lane, S. M. Grimes

Telephone:

(614) 593-1977

Scientific Staff:

T. N. Massey, F. Goeckner

FY 1992 Funding: $\quad \$ 330,000$

Research supported by this grant is in the area of neutron-induced and neutron-producing reactions relevant to the fusion energy program. Measurements include neutron elastic and inelastic differential cross sections as well as neutron-induced, charged-particle-producing reactions. Shell model calculations and/or R-Matrix analyses for the nuclei and reactions studied are also carried out to obtain the maximum anıunt of nuclear structure information from the data.

The ${ }^{9} \mathrm{Be}(\mathrm{n}, 2 \mathrm{n})$ reaction is important for neutron multiplication in fusion reactor breeding blankets. Preliminary combined shell model calculations for the ${ }^{10} \mathrm{Be}$ system and R-matrix calculations of the ${ }^{9} \mathrm{Be}+\mathrm{n}$ cross sections (based on the shell model predictions) have been completed, with the goal of improving the evaluations of the very important ${ }^{9} \mathrm{Be}(\mathrm{n}, 2 \mathrm{n})$ cross section.

As a test of our shell model calculations, the ${ }^{14} \mathrm{C}$ system has been studied. We have completed measurements on the $\mathrm{n}+{ }^{13} \mathrm{C}$ scattering to unbound inelastic levels. The experimental angular distributions have been compared to shell model-based R-Matrix predictions with reasonable agreement. An R-matrix fit to the data is in progress. We have begun a study of the alpha channel in the ${ }^{14} \mathrm{C}$ system by use of the ${ }^{10} \mathrm{Be}(\alpha, \mathrm{n}){ }^{13} \mathrm{C}$ reaction and find a large peak in the $0^{\circ}$ excitation curve. We plan several experiments to elucidate this structure.

To aid in interpreting these measurements and to facilitate predictions of such cross sections, we also are studying nuclear level densities. Calculations have been made to examine the role of the two-body forces in determining level densities and to study level densities for energies above $100 \mathrm{MeV}$.

Experiments have been done to examine the level spectrum at low energies by resolving all possible levels and infer level densities at higher energies through study of Ericson Fluctuations. 
Title of Project: Theory of Electron and Photon-Induced Reactions

\author{
Persons in Charge: $\quad$ D. S. Onley, L. E. Wright
}

Telephone Number: (614) 593-1721

Scientific Staff: $\quad$ Y. Jin, J-K. Zhang

FY 1992 Funding: $\$ \$ 126,000$

We provide theoretical guidance and analysis for investigations of electron and photon-induced reactions of elementary particles and atomic nuclei at medium energy (100-2000 MeV). In this work, we collaborate with experimental groups at electron laboratories throughout the world.

Interpretation of structure for nuclei requires detailed nuclear wavefunctions. We are engaged in calculations of nuclear structure, using a fundamental meson-exchange process to generate mean fields. We study hoth even and odd-A nuclei, and spontaneous deformation and polarization, implicit in the formalism. We have also looked at finite temperature nuclear states.

We are interested in the systematics of quasi-elastic electron scattering from all nuclei and what this reveals about nuclear structure. We study meson production in nuclei, including intermediate resonance states such as the delta and the role of quark structures in these reactions. Our preference is to use relativistic descriptions of all processes, but the possibility that there are simpler, equivalent nonrelativistic descriptions is also included.

New calculations of quasi-free scattering from ${ }^{40} \mathrm{Ca},{ }^{208} \mathrm{~Pb}$, and ${ }^{238} \mathrm{U}$ have shown that the existing ideas of nuclear structure and reactions account for the data very well. This is in contrast to earlier analyses. In photoproduction of pions in the delta region, we find moderate agreement with measurements in ${ }^{16} \mathrm{O}$ and ${ }^{12} \mathrm{C}$, although the possibility of modification of the delta particle in the nuclear medium would improve the agreement. More sensitive tests would require polarized photons, and this experiment is now scheduled. In studying a simple model of quark structure and reactions involving the strange quark, we have found that we are able to account for all measurements of the photoproduction of kaons at low energies, but we are limited by having a nonrelativistic model. 


\section{UNIVERSITY OF OREGON}

Institute of Theoretical Science and Department of Physics

Eugene, Oregon 97403

Title of Project: Relativistic Heavy-Ion Collisions

Person in Charge: $\quad$ R. C. Hwa

Telephone: $\quad$ (503) 346-5202

FY 1992 Funding: $\quad \$ 57,000$

An interesting and promising new development in high energy nuclear physics is the study of fractal properties of collision processes in which any particles are produced. It is an interdisciplinary area of research that applies the techniques of statistical physics and nonlinear systems to the complex problem of multiparticle production when nuclei collide. The goal is to find an efficient way of describing the complicated phenomena of the creation of hundreds, sometimes thousands, of particles so that the universal and important basic features can be exhibited more clearly. In so doing, one hopes to see signs of the creation of a new state of matter, the quark-gluon plasma, which is the ultimate goal of the heavy-ion program.

Recent advances have been made in two directions. One is in the development of a Monte Carlo code, called ECCO, for the simulation of pp, pA, and AA collisions. It contains the dynamics of branching in soft hadronic processes and is able to reproduce the data on intermittency. Universal fractal properties are found when applied to nuclear collisions. The other direction is in the study of second-order phase transition that could possibly take place before hadrons are formed. In the framework of Ginsburg-Landau, the factorial moments are found to possess a distinctive feature that resembles the critical behavior, in that a critical exponent can be determined possessing universal properties.

The results so far obtained offer the exciting possibility that intermittency analysis can serve as an effective method to examine how close relativisitic heavy-ion collisions are to the formation of thermalized quark matter. Seldom in the past could a numerical index be calculated in strong interaction physics that is independent of details, yet can be directly checked by experiments.

Much work remins to be done to complete the study of the critical index for intermittency and to gain a better understanding of the Ginsburg-Landau description of heavy-ion collisions. 
Department of Physics

Corvallis, Oregon 97331

Title of Project: On-Line Nuclear Orientation

Person in Charge: K. S. Krane

Telephone: $\quad$ (503) $737-4569$

FY 1992 Funding: $\quad \$ 78,000$

The research program has as its primary goal the study of nuclear structure through the spectroscopy of excited states and their radiations emitted in decay processes. The primary methods employed are decay spectroscopy of short-lived nuclei produced in heavy-ion reactions, low temperature nuclear orientation, and angular correlations.

Heavy-ion reactions are used to produce a selection of unstable nuclei. These nuclei are characterized by proton and neutron numbers significantly different from those of the stable nuclei found in nature. This results in an extreme instability of the nucleus to radioactive decay to more stable species; decay lifetimes are typically seconds to minutes, compared with hours to days for the radioactive species close to stability. These unstable nuclei are collected in an isotope separator, which selects a species of a particular mass number, and a beam of the selected nuclei is then transported to a helium dilution refrigerator, where they are implanted into a ferromagnetic environment at a temperature below $0.01 \mathrm{~K}$. At these temperatures, the nuclear spins align with the magnetic field of the ferromagnets, and the angular distribution of the radiation can be studied relative to the direction of alignment of the spins. Experiments are being conducted primarily at HHIRF (Oak Ridge).

These studies yield fundamental information on the properties and structure of nuclei. Our particular studies have concentrated on nuclei in the Au-Pt region and on the question of coexisting shapes in the low-lying structure. Our ability to understand nuclei depends on the success of various theoretical models in interpreting the observed structure, and data from these experiments contribute to these efforts, thus advancing our fundamental knowledge about nuclei and the forces responsible for their structure. 
Corvallis, Oregon 97331

Title of Project: $\quad$ Theoretical Studies in Nuclear Physics

Persons in Charge: R. H. Landau, V. A. Madsen

Tolephone: $\quad$ (503) $737-1693$

FY 1992 Funding: $\quad \$ 140,000$

The nuclear theory program has two tracts. One led by $\mathbf{R}$. Landau investigates the interactions of short-lived elementary particles with nucleons and nuclei. The other led by V. Madsen studies the low-lying collective excitations and giant resonances of neutrons and protons in nuclei.

Landau's research aims to use the interactions of elementary particles with nuclei to learn about unknown aspects of nuclear and particle physics. A quark model of the interactions of kaons with nucleons has been developed and tested by studying reactions with protons and heavy nuclei. This field theory model has a more microscopic basis than our previously developed potential models and draws conclusions as to the quark makeup of a number of resonances. We have continued to improve our momentum-space optical potential description of proton elastic scattering from spin $1 / 2$ nuclei and have applied it to the spin observables now being measured in polarized beam-polarized target experiments. We have tried in these projects to make use of and develop computational physics tools. This has been valuable training for our students and has contributed to the development of the field.

Madsen's research is aimed at probing nuclear structure via nuclear reactions. Current research includes determination of the densities of neutrons and protons responsible for transitions in nuclei and studies in which a proton gives its charge (and sometimes its spin) to the nucleus. The resulting densities are applied to systematics of electromagnetic and hadronic transitions. Recent work involves the connection between energy dependence of nuclear optical potentials and the nonlocality of the corresponding time-dependent potentials.

Carrying out these investigations often involves extensive computer calculations. We are aided in these tasks by an IBM RISC System 6000/530 with console and three X stations dedicated to use by the nuclear theory group. 
Title of Project: $\quad$ Studies of Target Fragmentation in Low Energy, Intermediate Energy, Relativistic, and Ultrarelativistic Nuclear Collisions

Person in Charge: W. Loveland

Telephone: $\quad$ (503) $737-7078$

Scientific Staff: $\quad$ A. Srivastava

FY 1992 Funding: $\quad \$ 99,000$

This program involves the study of the properties of the fragments of the target nucleus resulting from nucleus-nucleus collisions at low, intermediate, relativistic, and ultrarelativistic energies. We use either radiochemical techniques or time-of-flight methods to measure these properties. The target fragment properties are compared with models of nuclear reaction mechanisms to test them. Heavy target fragment yields are sensitive also to the competition between fission and particle emission. The observed values of the fragment properties are of practical significance in designing radioactive beam facilities and in assessing cosmic-ray or energetic particles interactions with matter.

Our experimental program has the following general thrusts: (1) we are evaluating new methods of synthesizing new heavy nuclei using radioactive beams; (2) in our intermediate energy research, we are focusing on the interactions of the heaviest projectile nuclei $(\mathrm{Kr}, \mathrm{Xe}, \mathrm{Au}$, and $\mathrm{U})$ with heavier nuclei, studying the evolution of damped collisions and fragmentation with increasing projectile energy; (3) in our studies of relativistic phenomena, we are involved in detailed measurements of spallation events to better understand the energy and momentum transfer in these collisions; and (4) we are further characterizing the properties of multifragmentation in ultrarelativistic nuclear collisions.

We have found large formation cross sections ( - barns) for heavy target residues in many intermediate energy reactions involving heavy target nuclei as part of our study of fission/particle emission competition, along with a decline in the fission cross section. We have found a transition from full to partial damping in $\mathrm{Xe}-\mathrm{Au}$ collisions as the projectile energy increases from 21 to $45 \mathrm{MeV} /$ nucleon.

Future work will focus on correlating the heavy residue properties with other observable features of the reactions through the use of multidetector arrays, heavy-ion storage rings, etc. 
Title of Project: Sudbury Neutrino Observatory

Person in Charge: E. W. Beier

Telephone:

(215) $898-5960$

Scientific Staff: $\quad$ W. Frati

FY 1992 Funding: $\quad \$ 297,000$

The Sudbury Neutrino Observatory is a second-generation solar neutrino experiment that will perform a real-time measurement of the interactions of neutrinos emitted in the beta decay of Boron 8 produced by nuclear fusion reactions deep inside the Sun. The measurements will be sensitive to possible transformations of neutrino type, which is direct evidence that neutrinos have mass. Non-zero mass for neutrinos would constitute a departure from the Standard Model of fundamental particle interactions, which to date can account for all experimental data on fundamental particles. This work is heing performed by a collaboration of physicists from Canada, the U.S., and the United Kingdom. The detector is being constructed in the Creighton Mine of INCO, Ltd., near Sudbury, Ontario, Canada.

The neutrino target is 1 kiloton of heavy water. Electrons produced by neutrino interactions with deuterons or with atomic electrons in the heavy water cause the emission of Cerenkov radiation, which is detected by an array of 9,500 photomultiplier tubes. The position, direction, and energy of the electrons is reconstructed from the time, charge, and geometrical location of the photomultiplier tubes participating in an event.

The funds allocated to the work performed under this grant support the construction of part of the signal precessing electronics for the photomultiplier tubes, the development and management of software for the project, and issues related to calibration of the detector.

Engineering design for the laboratory and detector was completed in 1992, excavation of the laboratory site will be completed in April 1993, and installation of detector components will begin in January 1994. The project is scheduled to turn on in calendar year 1995. 
Title of Project: Theoretical Research in Nuclear Structure and Nuclear Collective Motion

Person in Charge: A. Klein

Telephone:

(215) 898-8150

Scientific Staff: $\quad$ N. R. Walet

FY 1992 Funding: $\quad \$ 147,000$

The major goal of this research is to develop theories that account for the wide range of cooperative or collective behavior observed in medium and heavy nuclei, identifiable in limiting situations as rotations and vibrations of these systems. The major focus is on motions characterized by large deviations from the equilibrium configuration and by small velocities. Examples include large amplitude vibration and fusion reactions.

Several years ago, we completed the formulation of a new method for this problem, the generalized valley method. This serves to identify the propensities of nuclei to follow specified pathways away from equilibrium. This method has been applied successfully to problems of coupled vibrations. One current goal is to apply this theory to problems of increasing complexity and physical interest.

The theory involves the requantization of a classical theory, the latter derived from a many-particle quantum theory. As a second goal of the current work, we have developed and applied a systematic method of including quantum corrections in the dequantization procedure. For the immediate future, we plan to examine the coupling between collective and noncollective degrees of freedom, emphasizing those phenomena that fall under the rubric "Berry Phase."

During the past several years, major efforts have gone into other projects at best indirectly related to the major project described previously. For example, a complete review "Boson Mappings of Lie Algebras with Applications to Nuclear Physics" has appeared in Reviews of Modern Physics. New research stimulated by this work has been carried out and may continue for some time in the future.

In June 1992, a thesis, "Quantization of the Skyrmion," was completed in our group, a work that introduces the Kerman-Klein method of quantization, accompanied by a host of fresh ideas, for the study or this class of models.

Finally, a second thesis project, still in an early stage, also involves the application of the KermanKlein method, this time to the study of core-particle coupling in odd nuclei. The aim here is not only to study the interesting new phenomena at high spin, but also to re-examine some of the famous older problems in this field, such as attenuation of the Coriolis coupling. 
Title of Project: Studies of Relativistic Heavy-Ion Collisions at AGS

Person in Charge: W. E. Cleland

Telephone:

(412) 624-9059

Scientific Staff:

D. E. Kraus, E. M. Takagui, S. A. Voloshin

FY 1992 Funding: $\$ 243,000$

In experiment 814 , a study of both central and peripheral collisions of a $14.5 \mathrm{GeV} / \mathrm{A}$ silicon beam on a variety of nuclear targets has been carried out. The peripheral collisions are of interest to understand in detail the electromagnetic interactions of relativistic heavy ions. The central collisions are used to probe the excitation and subsequent disintegration of hot nuclear matter.

The University of Pittsburgh group has participated in the E814 Collaboration since its approval in 1985. This group has contributed to the experiment in several areas, including the construction and development of the trigger, the forward scintillation counter system, design and installation of the silicon multiplicity detector, and integration of the participant calorimeter into the experiment, including redesign of the monitoring and calibration systems for that detector. The analysis topics of this group include the kinematic reconstruction of peripheral collisions, studies of the multiplicity distributions, including measurements of the two-particle correlation function, and studies of transverse energy deposition in the central region.

Studies of the excitation energy distribution obtained from a full kinematic reconstruction of peripheral events with one proton in the final state (1p events) show very close agreement with the excitation energy distribution expected from low energy $(\gamma, p)$ measurements. These results were published in Physical Review C45, 2427 (1992). A more complete survey of these collisions, including studies of the $1 \mathrm{n}$ and $2 \mathrm{p}$ final states were presented at the Nuclear Physics Conference in Wiesbaden, Germany, in July 1992. Multiplicity studies were published in Physical Review C46, 312 (1992), and preliminary results for the two-particle correlation function were presented in a poster paper at Quark Matter '91. Preliminary results for the transverse energy measurement in the central region wett presented at the Washington APS meeting in April 1992. The analysis of all three of these topics is continuing.

The experimental apparatus is currently being reconfigured as E877, to study central collisions of Au beams. A preliminary run in spring 1992 permitted a first measurement of transverse energy distributions in these collisions, for which preliminary results were also presented at the Wiesbaden conference. 


\section{UNIVERSITY OF PITTSBURGH}

Department of Physics and Astronomy

Pittsburgh, Pennsylvania 15260

Title of Project: $\quad$ Silicon Drift Detector Studies for the STAR SVT

Person in Charge: T. J. Humanic

Telephone Number: (614) 292-4775

FY 1992 Funding: $\quad \$ 60,000$

One of the most exciting new directions in nuclear physics is the study of high energy heavy-ion collisions. In these collisions, nuclear matter might be heated and compressed to a sufficiently high degree that the nucleons melt into a macroscopic sea of constituent quarks and gluons called the quark-gluon plasma. Energies high enough to produce this new state of matter may exist at RHIC presently heing constructed at BNL.

One of the experiments slated to run at RHIC is the Solenoidal Tracker at RHIC (STAR) experiment, which is composed of two major detector systems to detect the particles emitted in these high energy collisions: TPC and Silicon Vertex Tracker (SVT). The current research program at Ohio State University is concentrating on the testing of Silicon Drift Detectors (SDD) which form the basis of the SVT. SDDs are novel devices that detect the two-dimensional position of a particle in a plane of thin silicon by the time it takes to drift electrons produced by the ionizing particle to a highly segmented anode at one end of the detector. We are bench testing SDDs with lasers and radioactive sources. Thus far, we have characterized the two-track resolution of a prototype device and its performance in a magnetic field.

Further work will focus on continuing magnetic measurements and studying temperature effects on the next generation of SDDs to be actually used in the STAR SDD. 
Titie of Project: $\quad$ Medium Energy Spin Physics with Lasers

Person in Charge: $\quad$ G. D. Cates

Telephone: $\quad$ (609) 258-4414

FY 1992 Funding: $\$ 109,000$

Many particles and nuclei possess the intrinsic property of spin, and many fundamental interactions depend upon the orientation (or polarization) of those spins. Lasers can be used in a variety of techniques for producing spin polarization, thus facilitating experiments that would otherwise be impractical. We are currently engaged in two experimental programs utilizing laser polarization techniques. In one experiment, we are studying atomic and nuclear spin interactions in muonic helium. In another experiment, we are studying the origin of the spin of the neutron.

At LAMPF we have been studying various laser techniques for producing polarized muonic helium. In addition to providing a better understanding of spin interactions at the atomic level, we have developed a practical means for producing high polarizations. We are now preparing to study the spin dependence of nuclear muon capture in ${ }^{3} \mathrm{He}$. At SLAC, we are studying the spin structure of the neutron using deep inelastic scattering of polarized electrons from a polarized ${ }^{3} \mathrm{He}$ target. The experiment tests the Bjorken and Ellis-Jaffe polarization sum rules, which are fundamental predictions of QCD.

Recent results include achieving the highest polarizations ever observed in muonic He, 30-50 percent. Also, we have developed a polarized ${ }^{3} \mathrm{He}$ target for use at SLAC, which is based on spin exchange with optically pumped $\mathrm{Rb}$ vapor. An unprecedented polarized target thickness of $7.5 \times 10^{21} \mathrm{~cm}^{-2}$ results from a length of $30 \mathrm{~cm}$ and a pressure of 10 atmospheres (at $20^{\circ} \mathrm{C}$ ).

Future work at LAMPF will focus on the measurement of $g_{P}$, the induced pseudoscalar form factor of the ${ }^{3} \mathrm{He}$ weak nuclear current. At SLAC, we began studying the spin structure of the neutron during the winter of 1992. 
Title of Project: $\quad$ Studies of Yrast and Continuum States in $A=100-200$ Nuclei

Person in Charge: $\quad$ P. J. Daly

Telephone:

(317) 494-5328

Scientific Staff: $\quad$ R. Broda, B. Fornal, Z. W. Grabowski

FY 1992 Funding: $\quad \$ 180,000$

This research program involves nuclear structure investigations by in-beam gamma-ray and electron spectroscopy following reactions induced by heavy ions. Goals of these investigations are to elucidate (1) the properties and structure of certain exotic nuclear species which are of special theoretical interest and (2) the structure of nuclei at very high angular momentum, with emphasis on nuclear superdeformation phenomena.

Most of our experiments are performed in collaboration with Argonne scientists at the Argonne Tandem Linear Accelerator System using the Compton-suppressed Ge spectrometer gamma-ray facility. Recent work has focused on gamma-ray studies of deep inelastic products of heavy-ion collisions; yield measurements for these reactions have demonstrated that yrast states in product nuclei extending over a broad range in $\mathrm{A}$ and $\mathrm{Z}$ are populated quite strongly, offering attractive opportunities for spectroscopic advances. Detailed measurements for the reaction ${ }^{124} \mathrm{Sn}+344 \mathrm{MeV}{ }^{80} \mathrm{Se}$ (15 percent above the barrier) have yielded extensive spectroscopic information about poorly known neutron-excessive nuclei in the neighborhood of $Z=50$.

Other recent work has included a study on long-lived isomers in proton-rich $\mathrm{N} \sim 82$ nuclei using the Argonne Fragment Mass Ánalyzer and a detailed investigation of the modes of populating and deexciting superdeformed bands in mercury nuclei. 
Title of Project: The Study of the Phase Structure of Hadronic Matter by Searching for the Deconfined Quark-Gluon Phase Transition Using 2-TeV Proton-Antiproton Collisions and by Searching for Critical Phenomena in an Exclusive Study of Multifragmentation Using $1 \mathrm{GeV} /$ Nucleon Heavy-Ion Collisions

Persons in Charge: A. S. Hirsch, R. P. Scharenberg, M. L. Tincknell

Tolephone: $\quad$ (317) 494-5390

FY 1992 Funding: $\$ \$ 459,000$

We propose to continue our exploration of the equation of state of strongly interacting matter. Using the 2-TeV proton-antiproton collider at Fermilab, we will study collisions that are capable of producing energy densities about 10 times that of normal nuclear matter. These events can produce large multiplicities of charged particles and may contain evidence that the deconfined phase of strongly interacting matter has been attained. This evidence may be contained in the behavior of the average transverse momentum and in the particle composition as a function of the charged-particle multiplicity.

We also propose to search for critical phenomena in the breakup of large nuclei using the Lawrence Berkeley Bevalac. Beams of gold, lanthanum, and krypton at an energy of $1 \mathrm{GeV} /$ nucleon will hombard targets of carbon and aluminum. The projectile fragments will be detected using a TPC, a multiple sampling ionization detector, and a time-of-flight detector. This will permit a complete reconstruction of the collision on an event-by-event basis. Evidence for critical behavior, indicative of a second-order phase transition, could manifest itself in the behavior of the moments of the observed fragment distribution. 
Department of Chemistry

West Lafayette, Indiana 47907

Title of Project: $\quad$ De-excitation Processes in Nuclear Reactions

Person in Charge: N. T. Porile

Telephone: (317) 494-5329

Scientific Staff: $\quad$ B. Srivastava

FY 1992 Funding: $\quad \$ 190,000$

The aim of this research is to study what happens to nuclei that have absorbed a lot of energy in their interaction with energetic particles. One possible response is the breakup of an excited nucleus into several smaller fragments. Another response, believed to be possible only at the highest available energies, is the formation of a quark-gluon plasma, which may have been the state of matter present in the universe during the initial moments following the Big Bang.

We are analyzing the results of a study of the particles created in the collision of $0.9-\mathrm{TeV}$ protons and antiprotons at Fermilab. We are looking for evidence for a change from normal nuclear matter to quark-gluon plasma by measuring the number and type of particles created in the collision, their energy and direction, and the correlation between these variables.

Multifragmentation is being studied at the Bevalac with the equation-of-state TPC. When used in conjunction with a multiple sampling ionization chamber and a time-of-flight wall, this detector will identify all the charged fragments emitted in a given interaction.

Continuing work on the multifragmentation will be performed during the coming year. The results will be analyzed in terms of a model that views multifragmentation as a "liquid-gas" phase transition. The critical exponents associated with this transition will be extracted from the data. 
Department of Physics

Troy, New York 12180

Title of Project: Theoretical Aspects of Electroweak and Other Interactions in Medium Energy Nuclear Physics

Person in Charge: $\quad$ N. C: Mukhopadhyay

Telephone: $\quad$ (518) 276-6663

FY 1992 Funding: $\quad \$ 75,000$

This project deals with the understanding of the electroweak structure of hadrons, in free state and in nuclear environment, thereby learning about manifestations of color interactions QCD in the nonperturbative domain. A particular focus of the project is the theory of electromagnetic excitation of haryon resonances, an important area of research at CEBAF, particularly in its Hall B program.

As an example, the Delta (1232) resonance is studied in processes involving pion photo- and electroproduction, Compton scattering, and neutrino scattering. From the Compton scattering, one discerns the important role of the Delta in controlling the nucleon magnetic polarizability, recently studied at Illinois and Mainz, and in the chiral perturbation theory. The issue as to whether the Roper (1440) resonance is a hybrid is another example, examined via its electromagnetic properties.

Connected with the baryon structure is the question of meson structure, addressed here in the framework of the dynamic symmetry, successful in molecular and nuclear physics. The group U(4) is used to describe the spatial degrees of freedom of the quark-antiquark color bond. The possible supersymmetric connection between mesonic and baryonic states is also a subject of investigation. In the complex nuclei, possible effects of physics beyond the Standard Model is examined in the context of search for the violation of the time-reversal invariance in muon capture. 


\section{RICE UNIVERSITY}

T. W. Bonner Nuclear Laboratory

Department of Physics

Houston, Texas 77251

Title of Project: Nuclear Structure at Intermediate Energies

Persons in Charge: $\quad$ B. E. Bonner, G. S. Mutchler

Telephone: $\quad$ (713) 5282159

Scientific Staff: $\quad$ S. Ahmad, J. A. Buchanan, J. M. Clement, I. M. Duck, I. Stancu, S. Toshkov

FY 1992 Funding: $\quad \$ 606,000$

Medium energy and nuclear physics has as its unifying theme the understanding of the strong interaction, that remnant of the color force between quarks and gluons, which spills out beyond the perimeter of individual particles and is responsible for, among other things, binding protons and l.eutrons together to form nuclei. At the Bonner Laboratory, we are deeply involved in this quest; our experimental program is focused on elucidating the origins and the consequences of this fundamental force of nature.

Recent examples of experiments that bear directly on our understanding of how particles are formed from the quark fragments of beam particles are our Brookhaven experiments using the polarized proton beam. By measuring the polarization of inclusively produced lambda and sigma particles and correlating this with the beam polarization, we have convincingly demonstrated just how the beam fragments combine with a strange quark from the sea to form these particles.

We are also involved in experiments that measure particles produced in heavy-ion collisions. Postulated signatures for a quark-gluon plasma, the predicted new state of matter, include anomalous production rates and polarization for strange particles. In another attempt to ignite the quark-gluon plasma, we bombarded nuclei with antiprotons. After the annihilation at the nuclear periphery, the forward-boosted products heat the nuclear matter beyond the annihilation site. Data are now being analyzed. We are also planning for RHIC as active members of the Solenoidal Tracker at RHIC Collaboration.

Recently, an experiment reported the almost heretical finding that the spin of the proton is not just the quantum mechanical sum of the spins of the constituent quarks. We mounted an experiment at CERN that will provide the definitive answer to this question. The experiment is timely, critically important, crisply defined, and ideally matched to the capabilities and interests of our group.

We just completed an experiment at Fermilab scattering $500 \mathrm{GeV}$ photons from the quarks and gluons inside nuclei. In an important future experiment, we will use the tagged photon beam at CEBAF to form excited hyperons. By observing their radiative decays, we probe the wavefunction of the constituent quarks. 
Title of Project: Nuclear Physics Research at the University of Richmond

Persons in Charge: $\quad$ M. F. Vineyard, G. P. Gilfoyle, R. W. Major

Tolephone:

(804) 289-8257

FY 1992 Funding: $\quad \$ 43,000$

The goal of this experimental research program is to study the atomic nucleus through scattering experiments using two different types of probes. One of these approaches involves the use of heavy-ion accelerators to investigate the interaction of one nucleus with another. The other technique is to study the electromagnetic response of the nucleus using CEBAF.

The heavy-ion work is focused on fusion reactions in light nuclear systems and the search for resonances in the interaction of one carbon nucleus with another. The fusion experiments have been performed at the ANL Argonne Tandem Line Accelerator System facility and the Florida State University Superconducting Accelerator Laboratory. The resonance experiments were conducted at the University of Pennsylvania's Tandem Accelerator Laboratory.

The projects in electromagnetic nuclear physics are performed as part of the CEBAF Large Acceptance Spectrometer (CLAS) collaboration. Our physics interests are in the photoproduction of mesons from nucleons and nuclear targets. We are involved in two approved proposals to study the photoproduction of low mass strange baryons and mesons. Currently, we are investigating the reasibility of using CLAS to identify etas produced on nuclear targets. Our contribution to the development of CLAS is the design, construction, and initial operation of the drift-chamber gas system. Last year, a prototype gas system was constructed and delivered to CEBAF. Current work includes the design of the final gas system and the development of the computer-control and gas-calibration subsystems. The delivery of the gas system to Hall B at CEBAF is scheduled for January 1995. 


\section{UNIVERSITY OF ROCHESTER}

Department of Physics and Astronomy

Rochester, New York 14627

Title of Project: Theoretical Nuclear Physics

Persons in Charge: J. B. French, D. S. Koltun

Telephone:

(716) $275-4359$

Scientific Staff: $\quad$ M. F. Jiang

FY 1992 Funding: $\quad \$ 156,000$

The general goal of this program is to advance the understanding of the dynamics of atomic nuclei in terms of their constituents, namely, nucleons (protons and neutrons), mesons, and quarks. To some extent, this corresponds to different levels of energies at which experiments may be done: nucleon dynamics dominates at low energies, meson physics becomes more important for higher energy studies (at accelerators such as LAMPF and AGS), and quarks may play a role in nuclei at even higher energies (SLAC, CEBAF, and RHIC).

Present research at the nucleon level concentrates on the spacings and widths of very complex energy level spectra in nuclei. The theory is a new type of statistical mechanics appropriate to small quantum systems such as complex nuclei. Among new results of this work are a sensitive limit on the possibility that nuclear forces exhibit a breaking of time-reversal invariance, a fundamental symmetry studied only with great difficulty; and an effective method for calculating nuclear state densities and smoothed partition functions.

Recent work at the meson level concentrates on nuclear reactions induced by beams of pi or $\mathrm{K}(+)$ mesons. We have produced new theoretical results on the role of meson exchange currents in double charge exchange reactions of pions and in elastic scattering of $\mathrm{K}(+)$ mesons.

Studies at the higher energy level have aimed at understanding how to analyze scattering experiments (e.g., electron scattering) in terms of separating the role of quarks from that of nucleons in nuclei. The techniques range from the use of specific quark models to a more general theory of the inelastic response function. 
Department of Chemistry

Rochester, New York 14627

Title of Project: $\quad$ Studies of Heavy-Ion Reactions and Transuranic Nuclei

Person in Charge: W. U. Schroder

Telephone: $\quad$ (716) $275-4231$

Scientific Staff: J. R. Huizenga, B. Lott, J. T_ke

FY 1992 Funding: $\quad \$ 330,000$

This experimental research program explores the dynamical behavior of hot and compressed nuclear matter and attempts to recreate in the laboratory controlled conditions such as may exist in the core of supernova explosions. Reactions between two complex nuclei at intermediate bombarding energies are used to investigate the mechanisms by which colliding nuclei interact; e.g., how they transfer mass, acquire high temperatures, experience nuclear fusion, and eventually disintegrate. Experiments search for evidence of a complete instantaneous disassembly into multiple small fragments predicted for nuclei heated beyond their stability limit.

In a first experiment of its kind, intermediate energy collisions between very heavy nuclei were studied with full coverage of all light particles, using a combination of 4-pi neutron and charged-particle detectors. In addition, exclusive measurements of associated neutrons were conducted for one of the heaviest available projectile-target systems. The spectacular results demonstrate surprisingly that binary, dissipative reactions prevail for all impact parameters. Of similarly high relevance for the development of reaction theory for the Fermi energy regime is the discovery that kinetic-energy damping in central collisions is incomplete. The observation made from these experiments, that emission patterns of intermediate mass fragments are distinctively different from those of light particles, will be helpful in resolving a long-standing puzzle in reaction studies involving lighter systems.

Progress has also been made in understanding the formation and disintegration process of composite nuclei produced at lower bombarding energies. It was found that discrepancies between fusion-evaporation reactions reported earlier are due to thermal differences in highly excited composite nuclei rather than a result of entrance-channel memory.

Future progress in understanding of reaction mechanisms in the Fermi energy regime is expected from calorimetric studies employing an advanced segmented neutron calorimeter currently under construction, in combination with multi-element charged-particle detectors. 


\section{RUTGERS UNIVERSITY}

Department of Physics and Astronomy

Piscataway, New Jersey 08855

Title of Project: Nuclear Structure Theory Relevant to Modern Probes

Person in Charge: $\quad$ L. Zamick

Telephone: $\quad$ (908) $932-3874$

FY 1992 Funding: $\quad \$ 65,000$

Whereas for many years problems in theoretical nuclear structure have been carried out using nonrelativistic equations, we are finding evidence where the relativistic approach in which a nucleon in the nucleus has a Dirac mass less than the free value leads to large changes. This is especially true for spin excitation rates. The effects of relativity on the spin-orbit and tensor interactions in nuclei are being carefully studied.

I am also studying various aspects of superdeformed states in nuclei such as quasi-molecular resonances, linear chain states, and the surprising appearance of identical bands in neighboring nuclei. I am finding that the deformation is approximately linear in the number of nucleons that is transferred from lower to higher shells.

I am finding relations between the spectra of even-even and neighboring even-odd nuclei in cases other than the above deformed identical bands. For example, in a two-to-one relationship, the excitation energies of double isobaric analogue states in selected even-even nuclei are surprisingly close to double the excitation energies of single analogue states in even-odd nuclei.

My long-standing interest in scissors mode excitations in nuclei from the shell model point of view has led me to develop a linear energy weighted sum rule that shows why the excitation rate to these orbital magnetic dipole states is proportional to the electric quadrupole excitations in the same nucleus.

In support of local experimental research on pion absorption in nuclei, I am examining the problem of why the pion absorption rate, followed by two nucleon emission, does not grow with mass numbers nearly as fast as the number'of quasi-deuteron states in the nucleus. 
Titlo of Project: Development of an Electronic Nuclear Database Environment for Desktop Computers

Person in Charge: $\quad$ C. A. Stone

Tolophono: $\quad$ (408) $924-4940$

FY 1992 Funding: $\quad \$ 55,000$

The U.S. DOF maintains an electronic database of evaluated nuclear structure properties. Information in this database is used to generate reference materials for the nuclear science community, most notably the journal Nuclear Data Sheets, and the book Table of Radioactive Isotopes. These publications provide a valuable resource for the nuclear science community. Our goal is to improve this resource by creating an electronic interface to the electronic nuclear structure properties for desktop computers.

We have developed a novel electronic database manager for the nuclear structure database. The first version of our software, known as MacNuclide, is undergoing beta testing and will be released later this year. A graphical chart of nuclides, an image familiar to all in the nuclear science community, is used to interact with the database. Complex database searches can be formulated and the results displayed as a color chart of nuclides. This image is also used to visualize complex, subtle trends in properties. We are using interapplication communication for scientists to output information to other programs or to read in new information as an ancillary database. This software thus becomes one of several tools in the large nuclear data environment.

Highly interactive level scheme displays will be implemented over the next yoar. Level schemes are used to order and visualize the complex decay processes of excited states and elucidate underlying properties of the nucleus. Functionality built into the first version of the software will evolve into a sophisticated database. When our project is complete, scientists will have the traditional reference materials available as an on-line reference along with a dynamic graphical interface that allows them to adapt the information to challenging experimental and theoretical problems. 
Department of Physics

Stanford, California 94305

Titlo of Project: Nuclear Research Using the Electromagnetic Probe

Person In Charge: $\quad$ Z.-E. Meziani

Telephone: $\quad$ (415) $725-2345$

FY 1992 Funding: $\quad \$ 87,000$

The research carried out in this project addresses two questions.

First, how do the intrinsic propertits of the constituents quarks contribute to the properties of the nucleon? Although we have a good idea on how the momentum and charge of the constituents are distributed, a mystery persists as of the magnetization composition (spin crisis in the parton model). Second, how do the nucleon' electromagnetic properties change in the nuclear medium? Beyond nucleon-nucleon correlations that exist in nuclei, it seems that modifying the nucleon electromagnetic properties in the nuclear medium gives a consistently better description to the data.

We are preparing the E142 experiment at SLAC to measure the spin structure function of the neutron using a longitudinally polarized electron heam and a high pressure polarized ${ }^{3} \mathrm{He}$ target. We have tested successfully a 2-meter-long gas threshold Cerenkov counter at BNL. Four of these Cerenkov counters are ready for the experiment. A conceptual design of the Compton polarimeter that will be used in Hall A at CEBAF for measuring the electron beam polarization to high accuracy has heen completed. This device is of paramount importance for the parity-violation experiments of Hall A at CEBAF hecause it is nondestructive to the electron beam for constant monitoring of its polarization.

New and unique results of separated transverse and longitudinal inclusive response functions have been obtained at $|q|=1 \mathrm{GeV} / \mathrm{c}$ for light nuclei $\left({ }^{3} \mathrm{He}\right.$ and $\left.{ }^{4} \mathrm{He}\right)$. A clear q evolution of these responses is ohserved when compared to the low momentum transfer data and the Coulomb sum rule saturates to the expected value for these light nuclei.

In the near future, our research will focus on performing the E142 experiment and preparing for the E143 experiment at SLAC. 
Title of Project: Spin-Dependent Phenomena in Medium Energy Physics

Person in Charge: $\quad$ P. A. Souder

Telephone: (315) 443-9102

Scientific Staff: $\quad$ R. Hólmes

FY 1992 Funding: $\quad \$ 169,000$

The spin direction of an electron, muon, or nucleus may have a significant effect on nuclear scattering or reactions. Polarizing these particles requires difficult technologies, but some important information about nuclear physics may be obtained only if these spin variables are controlled by the experimenters. Our program addresses this need.

An elusive but fundamental property of the nucleon, the induced pseudoscalar form factor, can be studied by measuring the spin dependence of the muon capture in helium-3. This experiment requires producing polarized muonic helium. Recently, we have developed a novel technique in which the muonic helium is neutralized by collisions with laser polarized $\mathrm{Rb}$ atoms. The resulting polarizations exceed 50 percent. We are presently developing a detector that will enable us to exploit our new method to make an accurate measurement of the induced pseudoscalar form factor.

We also are participating in an experiment at SLAC to measure the spin dependence of deep inelastic scattering of polarized electrons from polarized helium-3. The goal of the experiment is to determine the spin structure of the neutron, i.e., how the quarks of the neutron are aligned relative to the spin of the neutron as a whole, and to test the Bjorken sum rule, which is a fundamental prediction of the theory of QCD.

Finally, we are planning an experiment at CEBAF to use the Hall:A spectrometers to measure the parity-violating asymmetry in the scattering of polarized electrons from hydrogen and helium. This experiment isolates the contsibution of the weak interaction. As a consequence, it will determine, when combined with other data on the nucleon, the contribution of strange quarks to the static properties of the proton such as its charge radius. 
Physics Department

Philadelphia, Pennsylvania 19122

Title of Project: $\quad$ Experimental Investigations in Particle Physics at Intermediate Energies

Person in charge: V. L. Highland

Telephone: $\quad$ (215) 787-8479

Scientific Staff: $\quad$ L. B. Auerbach, S. H. Kettell, K. W. McFarlane

FY 1992 Funding: $\quad \$ 340,000$

Our program attempts to uncover new aspects of the physics of subnuclear particles by searching for discrepancies with the dominant and highly successful theory known as the "Standard Model." Where the theory makes either a very precise or a very sweeping prediction, an experiment can make a crucial test of it.

Our measurement of the pion beta-decay rate was an example of the former type of test, as is our recent measurement of the decay rate of the $\mathrm{K}$ meson into two muons. This result agrees well with the minimum required sy the theory. The main object of the latter experiment (BNL E791) was to search for the decay into a muon and electron. The theory, without convincing reason, flatly prohibits this reaction. Any observation of it would require a fundamental restructuring of theoretical ideas. Our measurement, the most sensitive ever done, still shows the absence of the reaction at the level of about thirty in a trillion.

Similarly, our experiment, Liquid Scintillator Neutrino Detector (LSND), at Los Alamos will look for the transformation of muon-type neutrinos into the electron type. This is not allowed by the Standard Model, but it would explain discrepancies in the flux of neutrinos reaching us from the Sun. The experiment will also measure the scattering cross section of neutrinos on carbon nuclei and on protons. Empirical cross sections are always of interest because calculation may be unreliable. The carbon cross section is needed by those persons calculating supernova bursts. The proton data will give insight into the proton structure. We have also mounted a search for the formation of a particle from six quarks, something expected theoretically but never yet seen.

Our immediate plan is to focus on bringing the large neutrino detector to the operational stage and to then exploit it to the maximum degree possible. We are also helping with the attempt to design a still more sensitive $\mathrm{K}$ decay experiment. 


\section{UNIVERSITY OF TENNESSEE}

Department of Physics

Knoxville, Tennessee 37996

Title of Project: Nuclear' Spectroscopic Studies

Persons in Charge: C. R. Bingham, L. L. Riedinger

Telephone: $\quad$ (615) 974-7802

Scientific Staff: $\quad$ M. W. Guidry, S. P. Sorensen, X. C. He, Z. P. Li, C. H. Yu, B. E. Zimmerman, Y. Di, R. W. Kincaid, J. Zhang

FY 1992 Funding: $\quad \$ 400,000$

The goal of this research is to study the structure of the nucleus by measuring its properties in extreme states. Two extremes are high rotational frequencies and an imbalance of protons and neutrons; the shape of the nucleus is very dependent on the rotational velocity and the nature of the internal excitation in the nucleus. A third extreme is nuclear matter at very high energy densities, a condition previously found only in the first moments of the universe. Our measurements of the properties of these extreme states provide more rigorous tests of the current nuclear structure models.

Our program has been heavily dependent on the use of the HHIRF at Oak Ridge. Our group has been active in the construction and use of a multidetector array to observe particles and gamma-rays from colliding heavy ions to study high spin states of nuclei and an isotope separator facility to study the structure of exotic nuclei at low spins and excitation energies. Nuclei at very high densities are studied using relativistic beams of oxygen and sulfur at CERN.

Observed nuclear properties are interpreted in terms of various nuclear models, incorporating both single nucleon behavior with collective degrees of freedom. One promising recent development in theory is the Fermion Dynamical Symmetry Model, in which our group has played an important role. The theoretical effort is greatly enhanced by eminent visitors to the Joint Institute of Heavy-Ion Research in Oak Ridge, which is partially funded through this contract.

During the next few years we will actively pursue low energy experimentation at Argonne, Indiana, Isolde in CERN and Berieley, and heavy-ion physics at relativistic energies at Brookhaven and CERN. We will also undertake a major developmental program with a view to exploiting new vistas in nuclear physics and astrophysics promised by the Radioactive Ion Beams Facility to begin operating at Oak Ridge in 1995, and by the new massively parallel supercomputers that are presently becoming available to us. 


\section{MIDDLE TENNESSEE STATE UNIVERSITY}

Department of Chemistry and Physics

Murfreesboro, Tennessee 37132

Title of Project: $\quad$ Nuclear Structure Studies via Neutron Interactions

Person in Charge: $\quad$ R. F. Carlton

Telephone: $\quad$ (615) $898-2130$

FY 1992 Funding: $\quad \$ 31,000$

High resolution neutron total cross sections for spin-zero nuclei make possible detailed nuclear structure investigations. Nuclei under investigation include ${ }^{54} \mathrm{Fe},{ }^{122,124} \mathrm{Sn},{ }^{48} \mathrm{Ca},{ }^{88} \mathrm{Sr}$, and ${ }^{90} \mathrm{Zr}$. Their structure is investigated in the framework of a dispersive optical model in which the real part of the optical potential contains contributions derived from the imaginary part of the potential through the dispersion relation.

High resolution neutron cross sections are measured up to $25 \mathrm{MeV}$ at the Oak Ridge Electron Linear Accelerator. Resonance analysis is performed in the $0-2 \mathrm{MeV}$ energy region, using the R-matrix formalism to obtain average scattering functions. High resolution elastic scattering measurements are used in combination with the total cross sections to identify the resonances by spin and parity. This permits the determination of ievel densities and statistical properties for each partial wave.

Comparison to the optical model is made by energy-averaging the scattering function for each partial wave. This program of study has found it possible to characterize the detailed high resolution information with global optical parameters deduced from extensive high-energy scattering data. These investigations may thus be used to provide additional constraints on the parameters of the optical model. The success of the dispersive optical model in previous studies of other neutron-nucleus systems and the present investigations suggest that the model can be the basis for a global description of the neutron-nucleus mean field that covers negative energies, the resonance region, and neutron energies up to $100 \mathrm{MeV}$. 


\section{TENNESSEE TECHNOLOGICAL UNIVERSITY}

Department of Physics

Cookeville, Tennessee 38505

Title of Project: $\quad$ Studies of Fluctuation Processes in Nuclear Collisions

Person in Charge: $\quad$ S. Ayik

Telephone:

(615) $372-3156$

FY 1992 Funding: $\quad \$ 29,000$

One of the recent challenges in nuclear physics is to understand properties of nuclear matter and those of hadronic interactions under extreme conditions of high densities and high temperatures. Heavy-ion collisions provide the only way to produce nuclear matter under these extreme conditions. In recent years, a lot $\mathrm{o}$ ? theoretical progress has been made in understanding and describing such reactions with energies up to a few $\mathrm{GeV}$ per nucleon. In particular, one-body transport models in a semiclassical form have been widely, and quite successfully, used for a simulation of such processes.

Although these one-body transport models provide a good description for the average properties of one-body observables, they fail to describe the dynamics of density fluctuations. As a result, the fluctuations processes such as multifragmentation, dispersion of observables, and multiplicity distributions of produced particles cannot be investigated on the basis of these models. In order to describe fluctuation processes in heavy-ion collisions, we have recently proposed an extension of onebody transport models by incorporating correlations into the equation of motion in a stochastic approximation and in a consistent manner with the fluctuation-dissipation theorem of statistical mechanics. This gives rise to stochastic transport models for describing the evolution of the fluctuating single-particle density in heavy-ion collisions. The major objective of this program has been to develop a method for obtaining numerical solutions of the stochastic transport equation in the semiclassical limit and to carry out various applications to heavy-ion collisions at intermediate energies. In particular, we investigated the influence of dynamical fluctuations on particle production mechanism at below threshold energies.

Future work will focus on a further development of the model by employing an appropriate quantal representation, which provides a better framework than the semiclassical treatment for investigating fragmentation processes. In addition, using a relativistic extension of the model, which is based on the Walecka-type effective field theory, we plan to investigate the effects of dynamical fluctuations on collective flow patterns and particle production mechanisms in high energy collisions. 


\section{TENNESSEE TECHNOLOGICAL UNIVERSITY}

Department of Physics

Cookeville, Tennessee 38505

Title of Project: $\quad$ Structure of Neutron-Rich Nuclei and Rare Electron Capture Processes

Persons in Charge: R. L. Kozub, M. M. Hindi

Telephone:

(615) $372-3483$

FY 1992 Funding: $\$ \$ 76,000$

One goal of this research is to acquire a better understanding of the structure of neutron-rich atomic nuclei. Experimental data on such nuclei are needed in order to provide better tests of theoretical models of nuclear structure. Another goal is to improve measurements of masses of neutrinos, nature's most elusive particles. In order to do this, it is necessary to acquire a better understanding of processes affiliated with neutrino emission, such as the electron capture (EC) process by which some proton-rich nuclei decay.

The research on the structure of neutron-rich nuclei has been centered primarily on the measurement of electromagnetic decay properties. Heavy-ion-induced reactions in reversed-kinematics mode are used to create the nuclei of interest. Experiments are performed using national facilities, such as the Argonne Tandem Linac Accelerator System (ATLAS) at ANL. Energy levels in the neutron-rich isotope potassium-43, which have high angular momenta, have recently been identified.

Studies of rare EC processes are carried out using the photon coincidence spectrometer facility at Tennessee Technological University to detect radiation from nuclei which decay by such a process. $\mathrm{X}$ rays and gamma-rays that are emitted following EC can be detected with this facility. The probability for double K-shell vacancy production in EC decay has been found to be greater than that predicted by prevailing theories for both cerium-139 and iodine-125. A search for evidence of heavy neutrinos in the internal bremsstrahlung EC spectrum of iodine-125 is in progress.

Current plans include using the Fragment Mass Analyzer at ATLAS to search for bound clusters of neutrons and to continue studies of neutron-rich nuclei. Preliminary work has been done on a new search for massive neutrinos, using techniques of both nuclear physics and surface science. 
Title of Project: $\quad$ Nuclear Structure Models: Applications and Developrnent

Person in Charge: $\quad$ P. B. Semmes

Telephone: (615) 372-3145

FY 1992 Funding: $\quad \$ 22,000$

Theoretical models are used to investigate the structure of atomic nuclei through detailed calculations of experimentally observable properties. Assumptions or approximations are required to obtain mathematically tractable models because the nucleons interact strongly through a force that is not well understood, and also because many particles can participate. Consequently, nuclear physics is phenomenological in its approach, and current theories are tested and refined by making detailed comparisons between model calculations and new experimental data. The main goal of this research is to investigate the low energy structure of nuclei through such calculations.

Current research is focused on applying models relevant for both high spin states (yrast and near yrast) and low spin, low energy states in nuclei far from stability. Two general classes of models are employed: particle-core coupling and cranking. The experimental observables that can be addressed include level energies, spins, parities, electromagnetic moments, and transition rates. For example, electromagnetic properties of superdeformed states are currently being studied. Also, rotational states in moderately deformed odd-odd nuclei (with an odd number of protons and neutrons) are being investigated with particular emphasis on perturbations of the energy levels (signature splitting) and the residual proton-neutron interaction.

One recent development is the prediction that magnetic dipole (M1) transitions should be observable in some rotational bands of superdeformed nuclei in the mercury region. Indirect evidence for M1 transitions was found in recent experimental data, and calculations suggest that the M1 gamma-rays should be directly observable with the next generation of detector arrays (e.g., Gammasphere). Such M1 properties would provide new insights into the details of the single particle orbitals at superdeformed shapes.

Future work will include a more systematic study of signature splitting in rotational odd-odd nuclei to clarify the effects of the Coriolis forces, triaxial nuclear shapes, and the residual proton-neutron interaction. 


\section{TENNESSEE TECHNOLOGICAL UNIVERSITY}

Department of Physics

Cookeville, Tennessee 38505

Title of Project: Proton Resonance Spectroscopy

Person in Charge: J. F. Shriner

Telephone: $\quad$ (615) 372-3480

FY 1992 Funding: $\quad \$ 30,000$

The study of chaotic behavior provides a unifying description of many systems across a wide variety of disciplines, including essentially all subfields of physics. In large scale (classical) systems, the appropriate description is generally well understood; however, in very small systems where quantum mechanics is necessary to understand the behavior, a number of unanswered questions about even the correct description of chaos remains. One method of studying chaos in these systems is to examine the statistical behavior of the energy levels. A related question, although also of major interest and importance in its own right, concerns the role and degree of broken symmetries in such systems. The goals of this program are to study such phenomena in nuclear physics via states which can be populated as low energy proton resonances.

We are currently preparing for a series of experiments at Triangle Universities Nuclear Laboratory to study states in ${ }^{30} \mathrm{P}$ via the ${ }^{29} \mathrm{Si}(\mathrm{p}, \gamma)$ reaction. Knowledge of these states will provide appropriate data for a statistical analysis, which should provide new inforniation about both chaos and the degree of isospin breaking in the ${ }^{30} \mathrm{p}$ system. New detectors and electronics have been acquired or ordered, and new software to handle the data acquisition is being developed.

We have also been studying the sensitivity to time-reversal-invariance (TRI) violation of detailed balance measurements in the neighborhood of interfering resonances. A recent study has suggested that such measurements might be especially sensitive. These ideas are being applied to the specific cases of $(p, \alpha)$ and $(\alpha, p)$ reactions. Using previously measured resonance parameters, we have established that the sensitivity of this type of measurement is strongly dependent on the speciíic resonances studied. The next step in this analysis will be to compare the sensitivity of this type of measurement to other methods that have been employed to study TRI violation in nuclear physics. 


\section{UNIVERSITY OF TEXAS ATAUSTIN}

Department of Physics

Austin, Texas 78712

Title of Project: $\quad$ Studies in Medium Energy Physics

Person in Charge: $\quad$ G. W. Hoffmann

Telephone: $\quad$ (512) $471-1769$

Scientific Staff: J. McDonough, L. Ray

FY 1992 Funding: $\quad \$ 255,000$

The research program includes two general types of experiments. The first involves elastic collisions of very fast protons with speeds up to 84 percent of the speed of light with nuclei. This process is reasonably well understood so that careful analysis of the data gives new knowledge of the structure of nuclei and the nuclear force inside nuclei. The second type of experiments study rare decay modes of the electrically neutral kaon, a subatomic particle. Searches for decays which are forbidden in physicists' most comprehensive theory of nature, the Standard Model, permit new tests of this theory anc the chance of a fundamental discovery.

This year, we conducted a high precision measurement of medium energy proton elastic scattering from nuclei. The experiment required the development of many novel techniques and specialized apparatus for carrying out an absolute measurement of the scattering to an accuracy of 1 percent. Careful theoretical analysis of the data will provide unambiguous tests of relativistic ar $\mathrm{d}$ nonrelativistic models of nuclear scattering with unprecedented clarity. Substantial progress was also made in the construction of our superconducting polarized nuclear target for use in hadron-scattering experiments next year. The purpose of these experiments and theoretical analysis is to study the poorly understood spin-dependent components of the nuclear force as they occur inside nuclei, study new aspects of nuclear structure, and search for evidence of relativistic dynamics in nuclei. A new limit of accuracy was completed this year in nur study of rare kaon decays, which tests the Standard Model at roughly 10 times the level of sensitivity of previous measurements. Intensive work has also been done to develop a new experiment that will increase the level of sensitivity to fundamentally new physics yet another factor of 10 or more. Development of this new experiment is expected to continue for another 2 years before new data acquisition commences. 


\section{UNIVERSITY OF TEXAS AT AUSTIN}

Department of Physics

Austin, Texas 78712

Title of Project: Investigations of Pion-Nucleus Interactions

Person in Charge: C. Moore

Tolephone: $\quad$ (512) $471-8877$

Scientific Staff: $\quad$ S. Mordechai

FY 1992 Funding: $\quad \$ 172,000$

This research program investigates properties of nuclei, using pions as a probe. Pions are the least massive particles that interact strongly with protons and neutrons, coalescing momer: arily with the proton (or neutron) to form a resonance called a delta. Positively charged pions $\left(\pi^{+}\right)$are 9 times more likely to form a delta with free protons than are negatively charged pions $\left(\pi^{-}\right)$, and vice versa for free neutrons. In our studies, we can therefore probe whether a given excited state in a nucleus mainly involves the excitations of protons or of neutrons. For instance, we have found a "pure neutron" excitation in ${ }^{13} \mathrm{C}$ and two "pure proton" excitations in ${ }^{15} \mathrm{~N}$.

A unique process possible with pions is to send a $\pi^{+}$into a nucleus and get a $\pi^{*}$ out or vice versa, which is called "double charge exchange." This process is not well understood; we have studied its dependence on target mass, incident pion energy, excitation energy of the final nucleus, and angle of emission of the outgoing pion. Because the flux of available pion beams is small compared to that for other strongly interacting probes, these measurements are tedious and time consuming; but, the data are full of surprises, and new theoretical approaches will be needed to describe many of these results, like two nucleon correlations in nuclei.

The double isovector giant dipole state has been observed for the first time in the $\left(\pi^{-}, \pi^{+}\right)$doublecharge-exchange reaction on ${ }^{13} \mathrm{C},{ }^{27} \mathrm{Al},{ }^{40} \mathrm{Ca},{ }^{56} \mathrm{Fe},{ }^{59} \mathrm{Co}$, and ${ }^{93} \mathrm{Nb}$. The resonances observed in the $\left(\pi^{-}, \pi^{+}\right)$reaction are closely related via Coulomb displacement energy and isospin symmetry to the resonances measured in the inverse $\left(\pi^{+}, \pi^{-}\right)$reaction on the same nuclei. The new observations provide a direct determination of the upper isospin component of the double giant dipole state, which is generally very weak in the $\left(\pi^{+}, \pi^{-}\right)$reaction. The comparison between the double dipole in the two double-charge-exchange modes gives valuable information of the isospin splitting and the Pauliblocking effects for isotensor transitions. 
Department of Physics

Austin, Texas 78712

Title of Project: $\quad$ Medium Energy Measurements of N-N Parameters

Person in Charge: $\quad$ P. J. Riley

Telephone: $\quad$ (512) 471-1152

Scientific Staff: $\quad$ G. Glass, K. H. McNaughton, C. Nguyen

FY 1992 Funding: $\quad \$ 218,000$

The aim of this research is the determination of the nucleon-nucleon amplitudes at medium energies. The required data include both elastic and inelastic scattering experiments and the measurement of polarization and polarization transfer parameters. We have completed a series of $p$-p elastic scattering measurements and believe that the measurements have allowed unique determination of the $\mathrm{I}=1$ elastic phase shifts. The final experiment, that of elastic p-p differential scattering cross section

measurements in the energy range $500-800 \mathrm{MeV}$ at LAMPF, achieved an absolute accuracy of about 1 percent. Recently, we have been carrying on extensive np elastic spin transfer measurements at LAMPF to allow the determination of the elastic isospin-zero $(\mathrm{I}=0)$ phase shifts and amplitudes in the energy range 500-800 MeV. The data are in excellent agreement with new phase shift solutions by Arndt, Bugg, Leluc, and Lehar. They report that with the inclusion of these new data, the solutions are well determined with a well-behaved error matrix for the first time in this energy range.

During the past year, our work has emphasized the fabrication and testing of a large (448 sense wire) cylindrical drift chamber, which will be used in inelastic np--->pp $\left(\pi^{-}\right)$measurements at LAMPF. We believe that measurements of the differential cross section and analyzing power will contribute significantly to the understanding of the $I=0$ nucleon-nucieon interaction and of pion production processes.

Finally, we are participating in a proposal at BNL to study very rare neutral kaon decays (BNL 871). A second-generation detector has been designed and is being fabricated; the first engineering run is scheduled for spring 1993. We expect to devote a large fraction of our efforts to this experiment in the next few years. 
Title of Project: Theoretical Nuclear Physics

Person in Charge: T. Udagawa

Telephone: (512) 471-1984

Scientific Staff: $\quad$ C. Y. Kim

FY 1992 Funding: $\$ \$ 90,000$

Our research program is, broadly, the development and application of low energy nuclear reaction theory and medium energy nuclear theory. An emphasis is placed on realistic analyses of experimental data. Our program thus involves development of computer programs for the extensive numerical calculations, as well as development and testing of underlying theoretical methods.

In the area of low energy nuclear reaction theory, we are working on and with theoretical methods that allow us to calculate cross sections for complete fusion and also various types of incomplete fusion reactions within the framework of direct reaction theory. Recent developments include: (1) the demonsuration that all important components of the total reaction cross sections can be calculated within the single framework of the direct nuclear reaction theory and (2) the determination of the fusion part of the imaginary optical potential from simultaneous analyses of fusion and elastic scattering cross section data.

In medium energy nuclear theory, we use a new powerful method recently developed by us for continuum random-phase-approximation calculations. Currently, we apply the method to study nuclear medium effects on delta states excited in nuclei by means of charge-exchange reactions. The longstanding problem of the observed shift in the spectrum of the charge-exchange reaction has successfully been explained in terms of the nuclear mean field and pion exchange type two-body interaction effects.

Future work will focus on medium energy nuclear physics, particularly the analysis of exclusive cross sections for decay products of the delta measured in coincidence with nuclear ejectiles. This work will also include a study of nuclear medium effects on hyperons created in nuclei. 
Title of Project: Cyclotron Facility

Person in Charge: J. B Natowitz

Tolephone:

(409) 845-1478

FY 1992 Funding: $\quad \$ 965,000$

The Cyclotron Institute operates a locally constructed K500 superconducting cyclotron. This cyclotron is designed to provide 110 to $160 \mathrm{MeV}$ deuterons and 220 to $320 \mathrm{MeV}$ particles, heavy-ion projectiles with energies up to $80 \mathrm{MeV} / \mathrm{A}$ for $\mathrm{A}<40$ and up to $8 \mathrm{MeV} / \mathrm{A}$ at $\mathrm{A}=238$. Using an electroncyclotron resonance ion source coupled to the cyclotron, experiments have been run using beams ranging frem 2.4 MeV/A to $65 \mathrm{MeV} / \mathrm{A}$ and with mass ranging from 2 to 129 . The highest energy per nucleon bean developed to date is $65 \mathrm{MeV} / \mathrm{A}$ deuterons. The highest total energy beam is $2.2 \mathrm{GeV}$ ${ }^{63} \mathrm{Cu}^{19+}$.

A $4 \pi$ neutron multiplicity detecticn system (the ne?tron ball) with associated hodoscope arrays for charged-particle detection is used for the study of medium energy heavy-ion reaction mechanisms. A large solid angle, moderate resolution proton spectrometer has been constructed. It is optimized for use with the $\mathrm{KSOC}$ in investigating $\Delta \mathrm{Tz}=+1$ Gamow-Teller strength with (d, $\left.{ }^{2} \mathrm{He}\right)$ and (n,p) reactions. The Momentum Achromat Recoil Spectrometer, a new design Recoil Mass Spectrometer (RMS), especially suited for studies of inverse reactions at $0^{\circ}$, is used for structure and reaction measurements. An array of $50 \mathrm{BaF}_{2}$ crystals (hexagonal $6.5 \mathrm{~cm}$ face to face and $20 \mathrm{~cm}$ long) is available for high energy photon measurements with good time resolution. The HI-LI particle detector system from ORNL, combined with a 96 detector plastic-CsI ball, provides nearly $4 \pi$ detection efficiency for charged particles. A high effciency superconducting $\beta$ spectrometer constructed at LLNL is in use for studying anomalous positron production in heavy-ion reactions. A large solid angle $\mathrm{K}=400$ multipoledipole-mulipole type spectrometer with high resolution, obtained from Oxford University, England, is currently being installed for spectroscopic studies. A major upgrade to the data acquisition and analysis iacilities is underway. 


\title{
TEXAS A\&M UNIVERSITY
}

\author{
Cyclotron Institute \\ College Station, Texas 77843
}

Title of Project: Nuclear Physics Research

Persons in Charge: C. Gagliardi, J. Natowitz, L. Northcliffe, R. Tribble, R. Schmitt, K. Wolf, S. J. Yennello, D. H. Youngblood

Telephone:

(409) $845-1411$

Sciontific Staff: $\quad$ J. Bronson, H. Dejbakhsh, J. Hagel, Y.-W. Lui, S. Shlomo, R. Wada

FY 1992 Funding: $\$ 652,000$

Fundamental Symmetries. The basic laws governing the nucleon-nucleon interactions in nuclear matter, as well as the subnucleon degrees of freedom, are explored. Measurements of polarization effects in the collisions of few-nucleon systems and a search for rare-decay processes of the positive muon (MEGA Collaboration) are presently underway, as well as a search for missing strength in nuclear modes of oscillation where spin-up and spin-down particles oscillate out of phase. Radiative cantare in few-nucleon systems will be explored using polarized deuteron beams.

Nuclear Structure. Nuclear structure studies at Texas A\&M are carried out using a variety of projectiles. Extensive studies of isoscalar giant resonances (collective vibrations involving most of the particles in a nucleus) are canied out. In addition to providing precise measurements of the nuclear compressibility, these experiments focus on searches for other nuclear vibrational modes and on measurements of the electromagnetic and particle decay of the resonances. Properties of nuclei far from stability are determined using the new magnetic spectrometers, Momentum Achromat Recoil Spectrometer and multipole-dipole-multipole. The structure of both neutron-rich and neutron-deficient nuclei will be determined by in beam ray spectroscopy.

Heavy-Ion Reactions. Intermediate energy heavy-ion projectiles are being used to produce highly excited or highly rotating nuclei. The earliest stages of the nuclear collisions are characterized through obsurvations of high energy gamma-rays and pions produced in the initial interactions. Measurements of nucleon and light-cluster emission are used to follow the excited system as it moves toward equilibrium. Observations of emitted particles and fragments are employed to probe the detailed characteristics of the equilibrated nuclei and establish their properties and details of their de-excitation modes. Both dynamically and statistically controlled de-excitation processes are explored. Projectile breakup and other reaction studies having important astrophysical applications are also studied. 
Title of Project: Photon Detector for MEGA

Persons in Charge: C. A. Gagliardi, R. E. Tribble

Telephone:

(409) $845-1411$

Scientific Staff: $\quad$ X. L. Tu

FY 1992 Funding: $\quad \$ 138,000$

One of the major challenges of particle and nuclear physics today is to understand the extent to which the minimal Standard Model successfully describes the interactions among quarks, leptons, and gluons and to determine the modifications needed to construct a complete theory of the elementary particles and their interactions. The MEGA experiment-with its goals of searching for the decay of the muon into an electron and a gamma-ray with a sensitivity of 6 parts in $10^{13}$ and of determining the Michel rho parameter in normal muon decay to 0.00075 -represents a major contribution to this effort. The decay experiment is a search for a process strictly forbidden by the Standard Model but is permitted in virtually every extension that has been proposed. Observing a non-zero branching ratio would have enormous consequences. Within the Standard Model with its maximal parity violation, the rho parameter is exactly 0.75 . Models with right-handed weak currents permit rho to deviate from this result. A measurement of rho to 0.00075 is sufficient to search for a 2 percent admixture of a righthanded W-boson in the normal left-handed W-boson.

The MEGA collaboration has been working on development and construction of the detector systems for these experiments for the past several years. Our group, together with our collaborators from the University of Houston, is responsible for the photon detector sysiem. It is a collection of three independent, concentric cylindrical pair spectrometers located within a superconducting solenoid magnet. Each pair spectrometer includes two $\mathrm{Pb}$ converter foils, an multi-wire proportional counter (MWPC) to identify the $\mathrm{Pb}$ layer where the conversion occurred, drift chambers to track the produced electron-positron pairs, and scintillators to determine the conversion time. The scintillators and MWPC also provide the information used by the first-stage trigger, while the drift chambers provide the information used by the second-stage trigger. We have now completed the detector development phase and constructed two of the three pair spectrometers. At present, they are in use at LAMPF for the initial physics data run. This run will include the rho measurement and will be sensitive to a muon to e-gamma branching ratio of less than 1 part in $10^{11}$. Meanwhile, the third-pair spectrometer is under construction. It will be installed at LAMPF this coming winter. Our run next summer will allow us to reach a sensitivity of 1 part in $10^{12}$, and a subsequent run will achieve our ultimate sensitivity. 


\section{TEXAS A\&M UNIVERSITY}

Department of Chemistry

College Station, Texas 77843

Title of Project: Pion Correlations and Calorimeter Design for High Energy Heavy-Ion Collisions

Person in Charge: $\quad$ K. L. Wolf

Telephone:

(409) $845-1411$

Scientific Staff: $\quad$ A. D. Chacon, R. K. Choudhury, M. Murray

FY 1992 Funding: $\$ \$ 165,000$

The properties of nuclear matter produced in high energy heavy-ion collisions provide the focus for this program. The production of compressed matter at densities similar to those in neutron stars should be possible with the current generation of accelerators. Higher energy colliding beams of heavy ions may allow future experiments to probe the properties of matter corresponding to the early universe. The aim of the research here is to perform experiments and develop instrumentation suitable for the investigation of the properties of nuclear matter at high density and high temperature.

Experiments currently focus on particle correlations from systems produced with heavy ions accelerated by the LBL Bevalac. Energies of 0.8 to $2.1 \mathrm{GeV} /$ nucleon and ions ranging in mass from ${ }^{40} \mathrm{Ca}$ to ${ }^{197} \mathrm{Au}$ are used with the method of identical particle interferometry to determine the sizes and shapes of reaction zones.

The research will be extended to higher energies at CERN with the lead beam upgrade in 1994. Identical particle interferometry for pions, kaons, protons, and antiprotons will be pursued within the NA44 collaboration. The development of instrumentation for present and future experiments includes sampling calorimetry with optical fiber readout and high resolution time-of-flight detectors. 
Department of Physics and Astronomy

Valparaiso, Indiana 46383

Title of Project: High Sensitivity Tests of the Standard Model for Electroweak Interactions

Person in Charge: $\quad$ D. D. Koetke

Telephone: (219) 464-5377

Scientific Staff: $\quad$ R. W. Manweiler, T. D. S. Stanislaus

FY 1992 Funding: $\quad \$ 145,000$

While the Standard Model, the present theory for weak and electromagnetic interactions, agrees remarkably well with experimental measurements, it is known to contain ad hoc assumptions whose origins are not understood from first principles. Unlike quarks, leptons from one family have never been observed to mix with those from another family, each apparently being separately conserved in nature. Consequently, for unknown reasons, the decay of a muon into an electron and a photon (with no neutrinos) is assumed to be strictly forbidden. Moreover, all neutrinos in the minimal Standard Model are assumed to be exactly massless, thereby preventing the spontaneous transition of an electron neutrino to a muon neutrino (neutrino oscillations).

These assumed features of the minimal Standard Model are among those being studied in high precision experiments carried out by this research program at LAMPF.

MEGA is an experiment to search for the muon decay to an electron and a photon (gamma). The final state branching ratio $(e+$ gamma $) /(e+$ neutrino + neutrino $)$ will be measured to approximately $10^{-13}$, or 2 orders of magnitude better than the present world limit. The collaboration has designed and successfully constructed and tested a set of nearly massless, cylindrical, high resolution, multiwire proportional chambers to detect the electron and a multilayer pair spectrometer, all in a $15 \mathrm{kG}$ superconducting solenoidal magnetic field. In 1992, we collected data at the design muon decay rate of $500 \mathrm{MHz}$ instantaneous. Valparaiso University is responsible for much of the analysis software for the experiment. Observation of this decay, or achieving this branching ratio limit, will significantly help to discriminate between various extensions to the minimal Standard Model.

Using the same apparatus, we will measure the Michel rho parameter for muon decays to a precision between 3 and 10 times better than the present measurement. This experiment will test the form of the fundamental electroweak interaction. Data for this experiment will be taken in 1992.

Neutrino studies will he done with the Large Scintillation Neutrino Detector, at LAMPF. This experiment is in the final phases of construction and will take data in 1993. In addition to the search for neutrino oscillations, numerous additional neutrino and neutrino-nucleus experiments will be done with this same apparatus. 


\section{VANDERBILT UNIVERSITY}

Department of Physics and Astronomy

Nashville, Tennessee 37235

Titlo of Project: $\quad$ Expẹirimental Nuclear Physics

Person in Charge: J. H. Hamilton

Telephone: (615) 322-2456

Scientific Staff: J. Kormicki, W. C. Ma, A. V. Ramayya, X. Zhao

FY 1992 Funding: $\quad \$ 164,000$

The program seeks to understand the behavior of nuclear matter under extreme conditions which do not occur in nuclei found in nature. These exotic nuclei have many fewer or more neutrons than stable elements and are produced in our work either in the collision of two heavy nuclei or the spontaneous fission of actinide nuclei. Studies of nuclei under these extreme conditions are yielding new insights and understandings into the behavior of nuclear matter. These studies are carried out at Oak Ridge and ANL which have special facilities like the University Isotope Separator-Oak Ridge (UNISOR) and Fragment Mass Analyzer. The group has led in the design and securing funds to build a new generation Recoil Mass Spectrometer (RMS) which can identify the $\mathrm{Z}$ and mass of reaction products. This RMS will open up studies of even more exotic nuclei still farther from stability with production cross sections up to 1,000 times smaller than presently accessible to study and so will be the centerpiece detector for the new radioactive ion beam facility now underway at ORNL. This new RMS will open up studies of presently inaccessible heavier nuclei including new $\mathrm{N}$ equal $\mathrm{Z}$ nuclei. These nuclei will probe new regions of nuclear matter with much stronger Coulomb repulsion that can alter presently used nuclear mean fields. Our research explores the variations in nuclear shapes with changes in neutron and proton numbers and has revealed the importance of gaps in the neutron and proton single-particle energies for deformed shapes. Evidence for a new structure with deformation intermediate between normal and superdeformed shapes was discovered in 1992. Recently, we have shown spontaneous fission (SF) can yield considerable information on high spin states in neutron-rich nuclei. We did the first SF of 242-Pu and plan studies of other exotic SF. 


\section{VANDERBILT UNIVERSITY}

Department of Physics and Astronomy

Nashville, Tennessee 37235

Title of Project: Intermediate and Relativistic Heavy-Ion Experimental Nuclear Physics

Person in Charge: $\quad$ C. F. Maguire

Telephone Number: (615) 322-2634

Scientific Staff: $\quad$ R. Tezkratt

FY 1992 Funding: $\quad \$ 150,000$

The program of intermediate and relativistic heavy-ion experimental nuclear physics at Vanderbilt University has as its unifying goal a better understanding of nuclear matter pushed to its extreme limits. The intermediate energy phase of this research is in the domain of heavy-ion beams with energies from 10 to $50 \mathrm{MeV}$ /nucleon which broadly encornpasses the Fermi velocity of nucleons in the nucleus. Collisions of these heavy-ion beams with fixed targets often produce fused, compound nuclei at such relatively high temperatures, compression, and angular momentum that a cohesive, selfcontained nucleus is barely sustainable. Indeed, this state of nuclear matter represents an ideal testing ground for the nuclear equation of state, the fundamental relationship between energy, temperature, and density for normal nuclear matter.

The relativistic heavy-ion program of research is even more ambitious. Its aim, to be accomplished using the RHIC accelerator now under construction at BNL, is the identified highest priority in heavyion nuclear physics: the formation and study of the quark-gluon plasma. This is a new phase of matter having been postulated to have existed for no more than a few microseconds after the Big $\mathrm{Bang}$, and more recently having been predicted to be recreated in the $100 \mathrm{GeV} /$ nucleon $\mathrm{Au}+\mathrm{Au}$ collisions made possible by RHIC. The discovery and understanding of the quark-gluon plasma would certainly rank among the most important achievements of science since the founding of quantum physics early this century.

For the past 2 years, we at Vanderbilt University have been very active members of what is now called the PHENIX Collaboration, one of the two major detectors approved for the inception of the RHIC experimental program slated for 1997. That collaboration has assigned to us the vitally important task of developing an integrated computer simulation program which will mirror as realistically as possible the experimental response of all the dozen different subsystems in what may ultimately be a $\$ 50$ million detector project. In this, the first ytar of our DOE sponsored grant, we have in fact supervised a subgroup of some 50 members of the collaboration and have coordinated the initial release of the complex computer program called PISA (PHENIX Integrated Simulation Application). The simulation effort will be carried out under our direction over the next several years and will evolve into a cornerstone of the whole PHENIX experimental data analysis system for unraveling the mysteries of the quark-gluon plasma.

The intermediate energy research will be carried out at the Texas A\&M cyclotron, and this constitutes an enhancement of the program in fusion studies which we have been carrying out with exclusive experiments during the past several years, first at ANL and more recently with the Heavy-Ion Light 
Ion (HILI) detector apparatus at ORNL. We have been funded to build a near full solid angle charged-particle detector, the "CsI Ball," to complement HILI and which is now being fabricated.

Research in this area will be carried out over the next several years in conjunction with members of the Oak Ridge staff, the Texas A\&M Cyclotron Institute, and the Autonomous University of Mexico. 
Department of Physics and Astronomy

Nashville, Tennessee 37235

Title of Project: Microscopic Heavy-Ion Theory

Persons in Chargo: V. E. Oberacker, A. S. Umar

Telephone:

(615) $322-2828$

FY 1992 Funding: $\$ \$ 64,000$

In many subfields of physics (e.g., atomic, col densed matter, nuclear, and particle physics), theoretical physicists face the same basic challenge: how to describe the features of many-particle systems at the quantum level in terms of more basic constituent particles. The goal of nuclear theory, in particular, is to describe individual nuclei and nuclear reactions in terms of strongly interacting constituent particles (such as protons and neutrons, hadrons and mesons, quarks and gluons). The necessary theoretical framework depends on the relative energy of the particles. Because of their inherent complexity, quantum mechanical many-body systems usually require a large computational effort (supercomputers and sophisticated visualization tools).

Our research involves theoretical studies that are strongly tied to experimental programs at several U.S. laboratory facilities. More specifically, we study low energy nuclear structure and reactions in terms of ; ull three-dimensional static and time-dependent Hartree-Fock calculations. Our goal is to predict t2. properties of new exotic nuclei far off the stability line which will be produced at the Radioactive Ion Beam Facility at ORNL. These studies are vital for both nuclear structure and astrophysics. At intermediate energies, we investigate the dynamics of muons in the Coulomb field of fissioning nuclei; the main goal here is to learn about the nature of nuclear dissipation in large amplitude collective motion. This project is related to experimental studies at LAMPF. In connection with RHIC at Brookhaven, we study nuclear collisions in terms of a dynamical string-parton model, and we investigate the nonperturbative electromagnetic production of lepton pairs which is the dominant background process in the search of the quark-gluon plasma, a new form of matter whose identification is the principal goal of RHIC. 
Department of Physics

Charlottesville, Virginia 22901

Titlo of Project: Polarization Degrees of Freedom in Electronuclear Coincidence Reactions

Person in Charge: $\quad$ R. W. Lourie

Telephone:

(804) 924-6809

Scientific Staff: $\quad$ S. Van Verst

FY 1992 Funding: $\$ \$ 83,000$

We have built a proton polarimeter for the one hundred inch proton spectrometer (OHIPS) spectrometer at the MIT-Bates Linear Accelerator Center. It will be used for determining the polarization of protons produced in electron-initiated nuclear reactions. This provides an entirely new capability for studying such reactions and, indeed, the structure of the proton itself. This results from the fact that subtle aspects of the physics produce a preferred direction for the spin and this information is lost if one averages over all possible spin directions as current detection systems do. Our polarimeter will operate over the full range of proton energies accessible at Bates, from 100$800 \mathrm{MeV}$.

A primary goal of this research is to achieve a more thorough understanding of the proton structure. In recent times, it has been revealed that the proton itself is made of even smaller objects, the socalled quarks. A very fundamental question is, therefore, how are these quarks arranged inside the proton? In simple terms, is the proton round or is it deformed? Although the deviation from sphericity is expected to be quite slight, it can have a large influence on the polarization of the proton after one scatters electrors from it. This question directly addresses the nature of the force between the quarks because different forces will produce different equilibrium shapes (wavefunctions).

The proton focal plane polarimeter will also be used for studies of the most elementary nuclear system, the deuteron, comprised of one proton and one neutron. This system is of fundamental interest in nuclear physics because, due to its relative simplicity, one can make the most direct connection between experiment and truly microscopic theoretical calculations. Elucidating the structure of this two-hody system is essential for our understanding of more complex systems. Possible extensions include measurement of deuteron polarization in electron-induced deuteron knockout from the few-body systems. Here, the goal would he to obtain a more detailed understanding of the two-hody correlations that lead to two nucleon ejection. 


\section{UNIVERSITY OF VIRGINIA}

Department of Physics

Charlottesville, Virginia 22901

Title of Project: Electron Interactions with Nuclei

Porsons in Charge: J. S. McCarthy, D. B. Day, D. C. Crabb

Telephone:

(804) 924-6783

Scientific Staff: $\quad$ O. Rondon, J. Mitchell, J. P. Chen

FY 1992 Funding: $\quad \$ 526,000$

Photons and electrons are two electromagnetic probes essential for testing and discriminating between the two models of the nucleus. The traditional model describes the nucleus as being composed of nucleons and mesons. In recent years, a description of the nucleus based on QCD has become recognized as more correct. The QCD model describes the nucleus in terms of quarks, which are more fundaiiental particles than the nucleons. The electromagnetic probe is particularly adept at studying the basic nuclear force. The aim of our research is to study the nuclear interaction at short distances, where the nucleon/meson description eventually gives way to the underlying quark description of the nucleons.

Increased emphasis has been placed on using the spin degrees of freedom in lepton-nucleus interactions to extract new information. These measurements are being pursued at CERN and SLAC-and represent a significant portion of our proposed experimental program at CEBAF. The following list summarizes the ongoing research program including experiments, equipment development, and new initiatives.

(1) The construction of high power polarized targets for the experimental programs at CEBAF and SLAC is well underway.

(2) The measurement of the deep inelastic spin structure functions of the proton and neutron continues at CERN. This involves the scattering of $100-200 \mathrm{GeV}$ polarized muons from both proton and deuteron targets. This will be the first data on the neutron, which is essential in clarifying the difficulties in understanding the spin constituents in the nucleon which arose from the European Muon Collaboration experiment.

(3) A new experiment to complete a definitive measurement of both spin structure functions, $g_{l}(x)$ and $g_{2}(x)$, has been approved hy SLAC. The experiment will use the polarized $\mathrm{NH}_{3}$ and $\mathrm{ND}_{3}$ targets being built at Virginia. Future measurements in the low $x$ region will require facility modifications in order to bring $50-\mathrm{GeV}$ polarized electrons into End Station A.

(4) A proposal to measure parity-violating elastic and inelastic scattering will be submitted to SLAC in the next month. The proposed experiment will be very sensitive to the possible role of strange quarks in the proton.

(5) A proposal to measure deep inelastic scattering from nuclei at $x>1$ has been submitted to SLAC (E145). This experiment includes an effort to measure color transparency in (e,e'p) at high momentum transfers. 
Department of Physics

Charlottesville, Virginia 22901

Title of Project: $\quad$ Experiments on the Nuclear Interactions of Pions and Electrons

Persons in Charge: R. C. Minehart, K. O. H. Ziock

Telephone: $\quad$ (804) 924-6785

Scientific Staff: $\quad$ L. C. Smith, B. Wright, W. Stephens

FY 1992 Funding: $\quad \$ 344,000$

Our program is primarily oriented towards: (1) the study of pion interactions in the lightest nuclei, (2) the study of some fundamental properties of particles, and (3) the study of the internal structure of nuclei and nucleons using energetic electron probes.

We have measured pion absorption in deuterium and the helium isotopes, most recently at the Clinton P. Anderson Meson Facility (LAMPF) using $500 \mathrm{MeV}$ pions incident on a liquid helium target, with a detector system consisting of a magnetic spectrometer in coincidence with an array of large scintillators capable of detecting both charged particles and neutrons. Preliminary results show no density dependent effects in two-nucleon pion absorption.

At LAMPF; we are collaborating on the following experiments: (1) measurement of threshold production of neutral pions in the interaction of charged pions with protons, in order to determine low energy properties of the pi-pi interaction, (2) the MEGA experiment to search for neutrinoless decay of a muon into an electron and a gamma-ray with a limit of detection of one part in $10^{13}$, and (3) a new measurement of the rho parameter in muon decay. Data-taking for the first was completed this summer, and data-taking for the other two is just starting. At the Paul Scherrer Institute (PSI) in Switzerland, we are working on a new

experiment to make a precise measurement of the pion beta decay rate. 'At Virginia, we continue to search for free quarks in ordinary matter, using a magnetic suspension technique to test for fraction residual charge on small steel balls.

At the SLAC laboratory, we will collaborate on an experiment to study the spin structure of the proton. For the longer term future, we are preparing a set of experiments to study the electromagnetic excitation of nucleon resonances at CEBAF. 
Department of Physics

Charlottesville, Virginia 22901

Title of Project: Nuclear Studies with Intermediate Energy Probes

Person in Charge: $\quad$ B. E. Norum

Tolephone: (804) 924-6789

FY 1992 Funding: $\quad \$ 130,000$

The pion plays a fundamental role in our understanding of atomic nuclei; it is the particle that mediates the nuclear force. Consequently, an understanding of the production and interaction of pions is crucial to our understanding of nuclear physics. The study of the electromagnetic production of pions is a principal focus of this program. A second focus is the study of nuclear structure using complementary information provided by different probes. In particular, we are studying both neutron and proton densities in nuclei using a combination of electron and proton scattering measurements.

We recently completed a study of threshold electroproduction of neutral pions, a process that should be precisely calculable starting from very basic assumptions. Previous photoproduction measurements had suggested a large discrepancy between theory and experiment. Our measurements, as well as reanalyses of the photoproduction data, indicated that no great discrepancy existed but that our understanding of this very basic process was incomplete. Continued studies are being pursued at both the Bates Linear Accelerator Center and National Institute for Nuclear Physics and High Energy Physics-Amsterdam (NIKHEF).

The mechanism by which pion's are produced in the interaction of photons with nuclei is also being studied. Measurements of the pheto-production of pions from ${ }^{13} \mathrm{C},{ }^{14} \mathrm{C}$, and ${ }^{15} \mathrm{~N}$ are currently underway at the Saskatchewan Accelerator Laboratory (Saskatoon, Canada). These studies focus on the role of the delta-resonance in the production process.

We are currently completing the analysis of both electron- and proton-scattering measurements of the calcium isotopes; $\mathrm{Ca}-40,42,44$. These measurements are yielding the most precise picture yet of both the proton and neutron densities of such nuclei. Similar measurements of ${ }^{14} \mathrm{C}$ are being pursued. 
Title of Project: Photon Interactions with Nucleons and Nuclei

Persons in Charge: $\quad$ R. M. Sealock, S. T. Thornton

Telephone: $\quad(804)$ 924-6568

Scientific Staff: $\quad$ S. D. Hoblit

FY 1992 Funding: $\quad \$ 106,000$

High energy photons and electrons can be used to probe the atomic nucleus and its constituent protons and neutrons at very short distance scales through the electromagnetic interaction. This interaction is relatively weak and therefore doesn't greatly disturb the system being examined. Measurements at short distances are required to clarify the nature of the nuclear force and to understand the transition from a description in terms of the exchange of mesons to one based on QCD.

The majority of our research is conducted at the Laser Electron Gamma Source (LEGS) at the National Synchrotron Light Source at BNL. We also contribute to the design and construction of experimental equipment for CEBAF and have a program of instrumentation development. LEGS research uses polarized photons to investigate the structure of the proton and few body nuclei. At CEBAF, we are part of the CEBAF Large Acceptance Spectrometer (CLAS) Collaboration and have responsibility for constructing various beamline instrumentation. Our instrumentation research investigates novel arrangements of waveshifting optical fibers and scintillators for the detection of neutral particles. These instruments will have applications to basic research in nuclear physics, e.g., neutron polarimetry, as well as to medical or industrial imaging with radiation.

Recent research at LEGS has shown that polarization measurements can be sensitive to an E2 component in delta excitation but that theoretical calculations of the dominant $\mathrm{M} 1$ component will have to be improved. $: \checkmark$ 'e have recently demonstrated that our scintillator/waveshifting fiber detector has the potential for submillimeter position resolution for low energy gamma-rays.

In the very near future, we will carry out more extensive measurements at LEGS of the deformation, if any, of the delta. Such a deformation would be a direct indication of a tensor component in the nuclear force. Other planned experiments at LEGS include studies of nucleon-nucleon correlations in nuclei. An approved experiment using the CLAS detector at CEBAF to study the momentum transfer dependence of delta excitation in nuclei is expected to be run in late 1994. 


\section{VIRGINIA POLYTECHNIC INSTITUTE AND STATE UNIVERSITY}

Department of Physics

Blacksburg, Virginia 24061

Title of Project: Partial Wave Analyses of Hadron Reactions Below $2 \mathrm{GeV}$

Porsons in Charge: R. A. Arndt, L. D. Roper

Telephone:

(703) 231-6544

Scientific Staff: $\quad$ R. Workman

FY 1992 Funding: $\quad \$ 152,000$

The main effort of our group is to correlate the vast amount of experimental information on strong interactions below $2 \mathrm{GeV}$ which has been gathered over the last 30 years at laboratories throughout the world into partial-wave representations. This process reduces the tens of thousands of measured data into physically meaningful scattering amplitudes which can then be used as input to disentangle more complicated, i.e., nuclear, processes. The primary reactions we study'are nucleon-nucleon, pion-nucleon, and kaon-nucleon, which are supplemented with pion-production processes using either pion or protor beams. The scattering amplitudes satisfy generally accepted theoretical concepts, such as conservation of mass/energy and angular momentum. The theoretical parameters are adjusted to fit the measured elasti. scattering data; the varied parameters are the partial-wave amplitudes, which are relared to the probability for scattering in a particular quantized angular momentum state. The problem reduces to a modeling exercise in which tens of thousands of experimental data points are fitt. 2 with 100-150 varisble parameters through a nonlinear representation. The process is very computer intensive and is continually evolving due to the ongoing process of data collection around the world. Our solutions, the scattering database, and the solutions of our principal competitors are made available to the physics community through a package of interactive computer programs and databases known collectively as Scattering Analysis Interactive Dial-In (SAID). This package can be arcessed by TELNET to VPI \& SU computers or obtained on a DEC-VAX computer backup tape. About 300 such tapes have been sent to laboratories around the world, but direct access is eliminating the need to send backup tapes. SAID is becoming the standard repository for the experimental understanding of the most basic hadronic reactions. 
Department of Physics

Blacksburg, Virginia 24061

Title of Project: Theoretical Studies in Few-Body Intermediaie Energy Physics

Person in Charge: T. Mizutani

Telephone: (703) 231-6544

FY 1992 Funding: $\$ \$ 4,000$

We have studied the semileptonic decays of heavy mesons within the relativistic quark model taking into account the confinement of light quarks. The form factors characterizing heavy-heavy and heavylight transitions were calculated. It was shown that the form factors of the heavy-heavy transitions in the heavy quark limit are expressed in terms of the universal function being dependent on the product of four velocities of initial and final states in accordance with the Isgur-Wise symmetry. The form factors of the heavy-light transitions are found to be monopole functions in this limit. The electron spectra and decay rates were also calculated. We have also studied the baryon form factors at low energies. Within the use of this approximation, the major low energy characteristics of baryons such as magnetic moments, electromagnetic radii, and form factors, strong form factors which are needed for description of nucleon-nucleon scattering, were calculated. We are presently calculating the meson production on nucleon and the electric and magnetic polarizabilities of the proton and neutron within the quark confinement model. 
Chemistry Department

St. Louis, Missouri 63130

Titie of Project:- "Studies of Complex Fragment Emission in Heavy-Ion Reactions

Persons in Charge: R. J. Charity, L. G. Sobotka

Tolephone: (314) 935-6578

FY 1992 Funding: $\quad \$ 117,000$

Our work involves both the study of low energy nuclear phenomenon and the study of intermediate energy heavy-ion nuclear reactions. We are interested in the dynamics of fission, which has been the premier example of large amplitude collective motion for more than 50 years, as well as the parameters that control this process when nuclei have unusual neutron-to-proton ratios. The study of both the dynamics of fission and the fission of exotic nuclear species at low energy is related to the study of the decay of nuclei that initially are highly excited. Our work in the intermediate energy domain has two foci. On the one hand, we desire to learn about the properties of nuclear matter under abnormal conditions, in this energy domain, predominately low densities. This purpose runs abreast of the second, which is the study of the relevant reaction mechanisms. The two objectives are inexorably linked because our experimental laboratory for studying nuclear matter properties is a dynamic one. We are forced to ask how nuclear matter properties; such as phase transitions, are reflected in the dynamics of the reactions. We are therefore compelled to study the reaction mechanisms themselves and thus the response characteristics of finite, perhaps nonequilibrium, strongly interacting systems.

This effort makes use of accelerators in the U.S. (primarily at ORNL and Michigan State University) and abroad (both in France and Germany). We have developed several devices: these include the Dwarf Ball and Wall and the MINIWALL for studies of complex intermediate energy reactions, the channel enhancer/selector of low energy spectroscopy, and a new design for an $\mathbf{x}$-ray detector for jeveloping an atomic clock for nuclear processes. The latter we plan to use to study the dynamics of near last chance fission and $\alpha$-decay. 
Chemistry Department

St. Louis, Missouri 63130

Titlo of Project: Investigations of Nuclear Structure and Nuclear Reactions Induced by Complex Projectiles

Person In Charge: $\quad$ D. G. Sarantites

Tolophono:

(314) 935-6504

Scientific Staff: $\quad$ J. Barreto, J. Elson, P.-F. Hua, J. Hood, A. Kirov, Z. Majka, N. G. Nicolis, D. W. Stracener

FY 1992 Funding: $\quad \$ 272,000$

The aims of this project are (1) to study the structure and shapes of hot, highly excited nuclei at high spin, (2) to study unusual behavior of nuclei produced in heavy-ion-induced reactions by combining atomic and nuclear spectroscopic methods, and (3) to investigate the mechanisms of heavy-ion reactions at low and intermediate energies. For these purposes, we develop and utilize unique and novel instrumentation.

We have been searching for large deformations in nuclei in the mass 80 region. There, superdeformation is induced only by centrifugal stretching and shell stabilization. We have employed total spectroscopy to study the excitation energy and spin dependence of the population of different rotational bands in ${ }^{82} \mathrm{Sr}$. We found an unexpected difference in the proton energy spectra and in excitation energy when the yrast and the ground bands are populated at high spin. We have utilized the atomic clock method for determining the lifetimes of subbarrier emission of $\alpha$ particles. These are obtained from the fraction of alphas that follows $\mathrm{x}$ ray emission from the initial compound nucleus. We have reinvestigated previous claims for the existence of entrance channel effects in the fusion of symmetric and asymmetric projectile-target combinations that produce compound nuclei at the same excitation energy ( $50 \mathrm{MeV})$. Such effects appeared to violate the Bohr independence hypothesis. We find that in the decay of ${ }^{164} \mathrm{Yb}^{*}$ (formed by ${ }^{16} \mathrm{O}+{ }^{148} \mathrm{Sm}$ and ${ }^{64} \mathrm{Ni}+{ }^{100} \mathrm{Mo}$ ) by $\alpha \times n$ emission, the observed differences are due to the breakup of ${ }^{16} \mathrm{O}$ into ${ }^{12} \mathrm{C}+\alpha$ prior to fusion. This prevents compound nucleus formation at the same excitation energy. For the xn exit channels, the mapping from orbital to residue spin to $\gamma$-ray multiplicity and coincidence fold is responsible for the purported entrance channel effects. We have studied the statistical emission of deuterons and tritons from highly excited compound nuclei. We find that pertinent transmissions coefficients should be obtained by excluding the large contribution from direct reactions in the optical model absorption cross section. Future work will concentrate on the quantitative interpretation of the previously mentioned findings and in new experiments to further elucidate our results. 


\section{UNIVERSITY OF WASHINGTON}

Institute for Nuclear Theory

Seattle, Washington 98195

Title of Project: Institute for Nuclear Theory

Persons in Charge: W. Haxton, E. Henley

Tolephone:

(206) 685-3971

Scientific Staff: $\quad$ G. Bertsch, T. Hatsuda, D. Harley, A. Horing, M. Luo, S. Pollock

FY 1992 Funding: $\$ 1,500,000$

The purpose of the Institute for Nuclear Theory (INT) is to bring together a br. ad range of physicists, primarily theorists, to exchange ideas and collaborate on chosen topics at the forefront of nuclear physics. The INT scientific staff (postdoctoral candidates, 5-year fellows, and permanent staff) also pursues its own research, largely independent of the visitor program.

During FY 1992, INT organized and oversaw three programs and one workshop. The first program (9/30/91-1/31/92) was on mesons and fields in nuclei. It concentrated on the origins of model r. Lronic Lagrangians and their applications in nuclear physics. Topics ranged from the domain of low energy nuclear physics (nuclear structure) to high energy, deep inelastic scattering, with special attention given to physics at contemporary and future meson factories, heavy-ion colliders, and electron accelerators. Two mini-workshops, one at the beginning of November and the other at the beginning of January, raised issues that set the stage for seminars and discussions for the following period. The first of these was on pions in nuclei and led to exchanges of ideas between meson-factory and heavy-ion theorists that dealt with common interests in meson dynamics up to energies of about $1 \mathrm{GeV}$. Following this workshop, the program concentrated on more specific issues of meson dynamics and on quantum hadrodynamics. The January workshop was on hadrons in nuclei. Theories of baryon structure and how one might explore medium modifications of excited mesons and baryons and their couplings were extensively discussed during the workshop and throughout the remainder of the program.

The second program (2/1/92-5/31/92) was on fundamental interactions in nuclei. It concentrated on aspects of symmetry relevant to nuclear physics. Considerable attention was focused on neutrino physics, CP/T violation, P-violation (particularly recent studies of neutron spin precession in nuclear transmission experiments), and chirality. The third program focused on strangeness in relativistic heavy-ion collisions, critical discussion of strangeness as a signature for quark-gluon-plasma formation, and the physics of hypernuclei (production mechanisms, structure, and decays). The discussions on strangeness and relativistic heavy-ion interactions focused on stability of strange quark matter, including dibaryons; weak decays of strangelets and multistrange hypernuclei; estimates of formation cross sections for multistrange systems in high energy heavy-ion collisions; and theoretical techniques for calculating strangeness production. The hypernuclear structure discussion includes: the $\Lambda$ and $\Sigma$ hyperon single-particle central and spin-orbit potentials, spin dependence of the residual $\Lambda \mathrm{N}$ interactions, the origin and role of three-body $\Lambda \mathrm{NN}$ forces, and the potential impact of high resolution experiments at CEBAF. 
Our first stand-alone workshop, on the mechanism of core-collapse supernovac, was held 1 week in February. Approximately 35 physicists participated. The topics covered included the status of hydrodynamic calcuations, the role of the nuclear equation of state, relevant weak interaction physics, and constraints on dirac neutrino masses and related particle physics.

Our FY 1993 planned programs are as follows: (1) Microscopic Nuclear Structure, September 8 to December 18, 1993; (2) Nuclear Physics in Atoms and Molecules, February 15 to May 31, 1993; and (3) Phenomenology of Lattice QCD, June 21 to September 3, 1993. 
Nuclear Physics Laboratory

Seattle, Washington 98195

Title of Project: Tandem and Superconducting Booster Accelerator Facility

Person in Charge: $\quad$ D. W. Storm

Telephone: $\quad$ (206) $543-4080$

Scientific Staff: $\quad$ T. A. Trainor, W. G. Weitkamp

FY 1992 Funding: $\quad \$ 1,175,000$

The Tandem/Superconducting Booster Accelerator Facility uses an FN tandem accelerator to inject a superconducting booster that provides an equivalent DC acceleration of up to $26 \mathrm{MV}$. The tandem operates from 0.5 to $9 \mathrm{MV}$ and can also act as a stand-alone accelerator. The superconducting booster consists of 38 quarter-wave resonators made of copper plated with lead. The resonators are held at liquid helium temperature where the lead is a superconductor. The first experiment using the booster occurred in 1987, and the machine has been running successfully since. The booster increases the energy of proton beams from 18 to $33 \mathrm{MeV}$ and the energy of heavy ions with mass of up to about 60 by about a factor of 4 , increasing, for example, oxygen from $60 \mathrm{MeV}$ to $225 \mathrm{MeV}$.

Five negative-ion sources feed the accelerator. These are a direct extraction ion source for gaseous elements such as hydrogen and oxygen, two sputter ion sources for solid elements, a helium ion source, and a polarized hydrogen ion source. The polarized source is of the crossed-beams type in which neutral polarized hydrogen atoms collide with neutral cesium atoms to produce polarized negative hydrogen ions.

The laboratory possesses a (primarily VAX) computer network. This network includes a VAX 780/11, five microVAX 3200/GPX, six microVAX 3100, and one microVAX 2000 computers. Three of the microVAX 3200 computers are used for CAMAC-based data acquisition, and one of the $3200 \mathrm{~s}$ is used for accelerator control. In addition, we have a DEC 3100 and two HP 9000/710 workstations running UNIX.

General purpose experimental equipment at the laboratory includes a large solid angle magnetic momentum filter/spectrograph with an 8-m isochronous flight path, an electrostatic deflector for the separation of heavy-ion fusion recoils from beam particles, and a variety of Compton-suppressed gamma-ray spectrometers. 


\section{UNIVERSITYOF WASHINGTON}

Nuclear Physics Laboratory

Seattle, Washington 98195

Title of Project: Heavy-Ion Research

Persons in Charge: R. Vandenbosch, K. A. Snover

Telephone: $\quad$ (206) $543-4080$

Scientific Staff: $\quad$ M. Kaplan, D. Prindle, D. Wells, D. Ye

FY 1992 Funding: $\$ 1,785,000$ (funding for this and the following two projects)

The fusion of two heavy nuclei at energies near or below the Coulomb interaction barrier is governed by quantum mechanical barrier penetration. Previous studies have shown that the probability for subbarrier fusion is much higher than one would expect from one-dimensional barrier penetration. This is attributed to coupling of other degrees of freedom to the relative motion degree of freedom. Previous work in our laboratory has shown the importance of shape degrees of freedom in enhancing subbarrier fusion. Further work is in progress to see if the observed enhancement can be attributed solely to the known shapes of target and projectile nuclei or whether other dynamic shape changes are important.

The propagation of one piece of nuclear matter through another piece of nuclear matter is a complex and not well-understood subject. Does nuclear matter dissolve completely when propagating through another nucleus, or does it propagate as clusters? One way to study this is to look at the yield of complex nuclei (deuterons, tritons, alphas) in the forward direction and compare this with the yield of single nucleons. We are studying particle yields for reactions induced by heavy ions whose velocity is varied from well below the Fermi velocity up to the Fermi velocity. Sizable yields of complex particles are observed, and we are presently trying to interpret these yields relative to single-nucleon yields.

The giant dipole resonance gamma decay following heavy-ion fusion is used to study highly excited hot nuclei. New information on spin and temperature induced deformation and, in light nuclei, the isospin purity of the highly excited nucleus can be obtained. We have demonstrated liquid drop mode!-like, rotation-induced spherical-to-oblate shape changes at high temperature $\mathrm{T} \sim 2 \mathrm{MeV}$ and moderate angular momentum. Recently, we have also obtained evidence for the oblate-to-triaxial shape changes as is expected at high angular momentum. A good theoretical understanding has been achieved, including the role of thermal shape fluctuations. We also search for the previously postulated persistence of large deformation in the fusion of heavy near-mass symmetric ions.

In light nuclei, we have demonstrated the restoration of isospin symmetric compound nuclear reactions at high excitation energies. We measured several nuclei to test the hypothesis of a constant, energy and mass-independent, isospin violating spreading width. 


\section{UNIVERSITY OF WASHINGTON}

Nuclear Physics Laboratory

Seattle, Washington 98195

Title of Project: Fundamental Symmetries

Person in Charge: $\quad$ E. G. Adelberger

Tolephone: $\quad$ (206) $543-4080$

Scientific Staff: $\quad$ L. DẻBraeckeleer, B. R. Heckel, P. V. Magnus, H. E. Swanson

Nuclei provide important microscopic laboratories for studies of fundamental interactions because (1) nuclei participate in all the fundamental forces, (2) we have powerful and precise techniques for studying nuclear symmetries, and (3) the wide variety of nuclear states provides filters that can amplify very small symmetry violations and isolate particular aspects of the interactions under study.

The weak interaction between nucleons, which can be detected only because it violates mirror reflection symmetry (parity), is particularly interesting because it probes the quark structures of hadrons and is currently the only practical way to see Z-zero exchange between quarks. We are preparing an experiment to measure the parity-violating interactions of neutrons with liquid helium and liquid hydrogen. These measurements should allow us to determine the component of the weak nuclear force that is sensitive to the neutral current interaction between quarks.

We are now conducting experiments to make precision tests of the vector-current conservation (VCC), second-class currents (SCC), and time-reversal invariance in nuclear beta decay. Our goal is to test, for the first time, the VCC and SCC symmetries down to the level of the expected isospin-violating effects and to test for time-reversal'violation in the induced weak currents (weak interaction effects not present between bare quarks, but produced by the str' nng interaction among the quarks).

We are investigating physics beyond the Standard Model by using highly sensitive torsion ${ }^{2}$ lances to search for new macroscopic interactions predicted by a variety of theoretical considerations (dilatation symmetry, quantum gravity, CP-violating axions, etc.), and we have already set stringent upper limits on such phenomena. We use a balance that rotates in the Earth's field to search for interactions with ranges greater than 1 meter. The results from this instrument have ruled out a proposed "fifth force," and a "quantum gravity" theory that predicted antiprotons should fall with an acceleration appreciably different from that of ordinary matter. A new instrument consisting of a stationary balance surrounded by a rotating 3 tonne source is being used to search for exotic forces with ranges down to $1 \mathrm{~cm}$.

The experimental studies involve work on accelerators, at reactors, and with nonaccelerator devices. The development of new techniques and instruments is emphasized because of the crucial role they play in our research. 


\section{UNIVERSITY OF WASHINGTON}

Nuclear Physics Laboratory

Seattle, Washington 98195

Titlo of Project: Relativistic Heavy Ions and High Energy Nuclear Physics

Persons in Charge: J. G. Cramer, C. E. Hyde-Wright, D. W. Storm, T. A. Trainor

Telephone: $\quad$ (206) $543-4080$

Scientific Staff: $\quad$ I. Halpern, D. Prindle, X. Zhu

We are using very energetic heavy ions to study the behavior of nuclei and their constituents at e"tremes of energy density. This work offers the possibility of producing a completely new state of matter, a quark-gluon plasma in which quarks are not confined. In CERN experiment NA35, $200 \mathrm{GeV} /$ nucleon sulfur ions are brought into collision with targets of $\mathrm{S}, \mathrm{Cu}, \mathrm{Ag}$, and $\mathrm{Au}$. 'The collision's many secondary particles (about 200) are principally pi and K mesons, which are trackéd using a TPC and analyzed for momentum and particle type. The high multiplicity of particles in a single event is a rich source of information on the character of the collisions. We employ pion interferometry to examine the size and conformation of the collision "fireball." The strange particles produced in the collision are analyzed to provide information on the energy density and baryon population of the collision. In addition to analyzing data from experiment NA35 (runs completed in 1991-1992) we are participating in the design and construction of CERN experiment NA49 (planned for 1994) and of the BNL/RHIC STAR detector system (planned for 1997).

Photoproduction of positive pions on nuclei has been measured using the tagger facility at Saskatchewan Accelerator Laboratory. We have studied the A dependence of the cross section, to obtain information about the mean free path for pions in nuclear matter. Similarly, a CEBAF photokaon production experiment on $\mathrm{He}(3 \& 4)$ and $\mathrm{C}$ will study kaon production via the (dominant) quasifree hyperon knockout mechanism. The integrated kaon yield in nuclei tests our understanding of the elementary operator. The ratio of positive to neutral kaons measures the contribution of (chargeexchange) final-state interactions.

We have measured, at SLAC, elastic and inelastic electron scattering from the proton and deuteron and, at Bates, polarized inelastic electron scattering from deuterium with coincident detection of the the recoil neutron polarization. These experiments isolate the electric and magnetic form factors of the neutron, as a function of momentum transfer. A proposed SLAC experiment would measure high energy, high momentum transfer elastic photon scattering from the proton. Dimensional counting arguments predict a scaling law at fixed scattering angle for the cross section as a function of incident photon energy. Verification of this scaling law tests the perturbative QCD prediction that the cross section is dominated by proton configurations with just three (current) quarks. 
Department of Physics

Seattle, Washington 98195

Title of Project: Theoretical Nuclear Physics

Person in Charge: $\quad$ G. A. Miller

Telephone:

(206) 543-2995

Scientific Staff: $\quad$ M. Alberg, S. Bass, J. Cooperstein, T. Hatsuda, W. C. Haxton, E. M. Henley, S. Huang, W. Köpf, W. Lin, M. Lutz, G. A. Miller, L. Wilets

FY 1992 Funding: $\quad \$ 597,000$

The objective of nuclear theory is to provide a fundamental understanding of nuclei and to apply this knowledge to problems in related subfields. Our group is known for examining many areas: hadronic structure, fundamental symmetries, nuclear structure, high energy probes, and nuclear astrophysics.

Our efforts to understand nuclei center on QCD. QCD sum rules are used to describe static nucleon properties, parity-violating meson-nucleon coupling constants, and the density and temperature dependence of properties of nuclear hadrons. The QCD prediction that the nuclear optical potential may vanish for certain high momentum transfer reactions (color transparency) is being investigated. Exact numerical solutions of QCD using lattice gauge theory are sought. The confinement property is modeled in the QCD Lagrangian by approximating the long-range, non-Abelian part of the gluonic self-interactions and also maintaining chiral symmetry (chromodielectric model).

Tests of fundamental symmetries involve studies of nuclear anapole moments (caused by weak corrections to electromagnetic vertices), time-reversal violation in nuclear beta decays and nonleptonic hyperon decays, rho-omega mixing, nuclear structure effects on atomic parity violation, and neutrino properties in double beta decay.

The nuclear astrophysics involves solar model and particle physics solutions to the solar neutrino puzzle, stellar cooling as a test of new particle physics, and core-collapse supernova mechanisms.

There has been much recent progress. The chromodielectric model of the pion was derived and will be extended to other hadrons and their interactions. Rho-omega mixing was identified as strong enough to explain an old problem in understanding binding energy differences between mirror nuclei. A new method to compute the energy dependence of color transparency effects was developed and will be used to analyze and plan a diverse set of experiments. New restrictions on axion models and on neutrino oscillations were obtained from nuclear astrophysics. Effective ways to measure the amount of nucleonic strange quarks were developed. 
Department of Physics and Astronomy

Detroit, Michigan 48202

Title of Project: Relativistic Heavy-Ion Research

Person in Charge: T. M. Cormier

Telephone: (313) $577-2720$

Scientific Staff: $\quad$ R. Bellwied, S. Bennett, J. Hall, Q. Li, C. Pruneau

FY 1992 Funding: $\$ \$ 150,000$

Our research group uses relativistic heavy-ion beams from the BNL AGS. Gold beams, up to 2.3 $\mathrm{TeV} / \mathrm{c}$, interacting with heavy targets such as lead, produce potentially new forms of nuclear matter at unprecedented densities and temperatures.

At AGS, we are collaborators in the series of experiments E814, E877. This series of experiments uses a large forward spectrometer in conjunction with nearly $4 \pi$ calorimetry to measure the characteristics of particle spectra produced in central relativistic nucleus-nucleus collisions.

Our research group has been mainly interested in studying the flux of relatively low energy particles observed at large angles in a kinematic domain where multiple scattering in the hot dense matter of the collision zone is the dominant source of particles. These multiple scattered particles reveal features of the degree of thermal equilibration of the intermediate state. In the same experiments, our research group studies the general spatial distribution of the outward energy flow from the collision. Using the $4 \pi$ calorimetry of the E877 Experiment, we are particulary interested in observing possible coherent flow patterns suggestive of hydrodynamic processes in central Gold on Gold collisions.

In a parallel effort at AGS, we are participating in the development of experiment E864. This experiment is a large acceptance forward angle spectrometer of great sensitivity designed to look specifically for heavy exotic objects which might be produced in relativistic Gold on Gold collisions. The likelihood that such objects will be produced is greatly influenced by the production of a quarkgluon plasma in an intermediate state of the collision. Thus, in addition to providing a general search for exotic heavy objects, this experiment will also provide a platform for a very sensitive search for phenomena associated with the production of the quark-gluon plasma.

Finally, our group participates in the preparation for the STAR Experiment at RHIC. In this collaboration, we presently have project management responsibility for the silicon vertex tracker, a device based on the relatively new technology of silicon drift detectors. An aggressive research and development program is underway with the goal of delivering a silicon tracking device for use in the first collisions at RHIC in 1997. 
Department of Physics

Williamsburg, Virginia 23187

Title of Project: $\quad$ Topics in Theoretical Physics

Person in Charge: F. Gross

Telephone:

(804) 221-3516

Scientific Staff: $\quad$ J. Milana, A. Stadler, Y. Surya, R. Kahler

FY 1992 Funding: $\$ \$ 92,000$

Nuclear matter is composed of quarks, antiquarks, and gluons, but it is still not known how to describe its structure in terms of these constituents. The objective of this research program is to construct models for the forces that bind these constituents together and to use these models to calculate the structure of the simplest nuclear and subnuclear systems, including the pion, the nucleon, and "few body" nuclei (nuclei with only 2-4 nucleons). The goal is to find the correct forces by comparing the predicted results with experiment.

Recently, a new model of mesons (assumed to be quark-antiquark bound states) has been developed. The model has a linear confining interaction which is both covariant and consistent with chiral symmetry, and it automatically gives a zero-mass pion in the chiral limit, even if the linear part of the confining interaction is a pure scalar. The model is currently being used to predict the properties of the meson spectrum. In a second work, a new covariant calculation of pion-nucleon scattering has been completed. The model is so simple that it can be incorporated consistently into a relativistic meson theory of nuclear forces.

Work is beginning on two new problems. Meson exchange forces are being calculated from a chiral bag model, which should give a simple picture of how such forces are modified by the structure of the nucleon. The structure of the ${ }^{3} \mathrm{H}$ bound state will be calculated from a fully covariant meson exchange formalism, and the size and importance of relativistic effects will be studied. 
Department of Physics

Madison, Wisconsin 53706

Title of Project: Development of a Hydrogen and Deuterium Polarized Gas Target for Applications in Storage Rings

Person in Charge: W. Haeberli

Telephone: $\quad$ (608) 262-0009

FY 1992 Funding: $\quad \$ 132,000$

The project concerns a new method to study the spin dependence of nuclear reactions. A low pressure target of spin-polarized hydrogen or deuterium will be placed in a storage ring, where the intense circulating beam of charged particles (electrons, protons, antiprotons) circulating in the ring interacts with the polarized larget. This technique will yield results much superior to conventional solid polarized targets, provided it is possible to achieve a target thickness of about $10^{14}$ polarized atoms $/ \mathrm{cm}^{2}$ with a target polarization above 80 percent. Because the density of polarized gas produced in free heams of atoms is about 100 times too low, in the present project, the atoms are injected into narrow, long vessels (storage cells) into which the free beam is injected.

The project has been focused on the development of wall coatings suitable to prevent depolarization of the atoms during the roughly 1,000 wall collisions they suffer while in the cell. The wall coatings should be resistant to radiation damage and should permit operation of the storage cell at low temperatures because cooling the cell increases the target thickness.

A number of wall coatings has been discovered which give excellent polarization retention down to $80 \mathrm{~K}$ absolute temperature. A recent test of a polarized hydrogen target cell in a proton storage ring was performed, which shows that for an aluminum cell coated with a high temperature version of Teflon, more than 90 percent of the polarization is retained after 500 wall collisions. A test with circulating proton beams up to $1 \mathrm{~mA}$ showed no deterioration of the target polarization after 100 hours of bombardment.

A pilot experiment is in preparation for installation of a target in the Indiana Storage Ring to demonstrate the feasibility of spin correlation measurements by this technique. Methods developed during the present projects will have further applications as the polarimeter of a target High Energy Resolution Array (HERA) to be installed in the electron ring. 
Department of Physics and Astronomy

Laramie, Wyoming 82071

Titie of Project: Muonic Hydrogen Production and Kaonic Atoms

Person ir. Charge: $\quad$ R. Kunselman

Tolephone: (307) 766-6150

FY 1992 Funding: $\$ 31,000$

The research program has two main areas of interest with exotic atoms. The first is with muonic hydrogen isotopes in vacuum, and the second is with kaonic atoms.

The muonium production method has been extended to a study of muonic hydrogen, muonic deutcrium, and muonic tritium in a vacuum. A proton, deuteron, or triton is captured by a negative muon in a solid ice target and drifts into a vacuum region where production rates and precision energies of interest in fusion and quantum electrodynamics are studied.

The efforts on kaonic atoms are to measure the atomic $x$ rays from atoms formed with the negatively chargci kaons. $T \cdot x$ rays are studied to determine nuclear distributions, to determine kaon-nuclear interaction parameters, and to test energy calculations. The $x$ rays from sigmonic atoms are produced as a byproduct from kaon capture and are studied to determine the sigma-minus magnetic moment and mass. This effort is developing as the KAON factory at the Tri-University Meson Facility (TRIUMF) is developing. The first emphasis will be to measure the energy and line width of the $2 p-1$ s transition of kaonic hydrogen which is directly related to the fundamental kaon-nucleon scattering length. 
YALE UNIVERSITY

A.W. Wright Nuclear Structure Laboratory

New Haven, Connecticut 06511

Thlo of Project: $\quad$ ESTU Tandem Accelerator

Person in Chargo: $\quad$ H. R. Hyder

Tolephone: $\quad$ (203) $432-3090$

FY 1992 Funding: $\quad \$ 1,525,000$

The ESTU tandem accelerator operates at terminal voltages between 4 and $21 \mathrm{MV}$ to provide beams ranging from protons to gold at energies from 4 to $350 \mathrm{MeV}$ or higher. These beams are used by Yale scientific staff and visiting groups from universities and national laboratories. The tandem runs on a 24-hour 5-day schedule and during the 12-month period ending in August 1992 was at voltage for 5,180 hours. Beam was available for 4,071 hours and on target for 3,220 hours.

A new negative ion injector, including an inflector magnet with $90^{\circ}$ analysis and a mass resolution of $\Delta M / M=1 / 250$, a high intensity Hé source, a sputter source, and enhanced vacuum and computer controls, is scheduled for installation in fall 1992. New equipment for the high voltage terminal, including two foil strippers and a recirculating gas stripper followed by an electrostatic quadrupole charge-state separator, is undergoing bench tests prior to installation. The PC-based computer control system has been upgraded with a faster processor and more powerful software and will be enhanced with a VME link to the new terminal equipment. These changes will lead to higher energies and intensities of heavy-ion beams by the use of double stripping.

The major ancillary equipment associated with the ESTU accelerator includes (1) a large solid-angle (12.6 msr) Enge split-pole magnetic spectrometer which was assembled and commissioned in parallel with the accelerator, (2) a comprehensive, integrated array of gamma-ray and x-ray detectors, including 5 (23 percent) Ge detectors with BGO Compton-suppression shields, 5 low energy photon spectrometers, an array of 38 BGO detectors operating as a multiplicity filter, one large ( 72 percent) Ge detector and a 4-crystal Compton polarimeter, (3) a segmented hemispherical array of 35 neutron detectors with $\gamma$-n discrimination, and (4) a large scattering chamber with a fast arm for out-of-beam studies of delayed activities, with half-lives as short as $500 \mathrm{~ms}$. 


\section{YALE UNIVERSITY}

\section{A.W. Wrigint Nuclear Structure Laboratory}

New Haven, Connecticut 06511

Titie of Project: $\quad$ Structure of Exotic Nuclei

\section{Porson in Chargo: $\quad$ C. J. Lister}

Tolephono: (203) 432-3090

\section{Scientific Staff: $\quad$ P. Chowdhury, S. J. Freeman}

FY 1992 Funding: $\$ \$ 1,545,000$ (For this and the following three projects)

The study of exotic nuclei at the limits of stability is fundamental to the development of a universal description of all bound nuclear systems. Understanding the response of nuclei to nonequilibrium conditions can extend our knowledge of the nuclear state far beyond the stable and near-stable isotopes which presently surround us on Earth and is relevant to the hostile environments in the universe both now and at earlier times. The limits of stability are diverse and can be explored when creating nuclei with unusual neutron-to-proton ratios, when creating nuclei with high internal excitation energy or high angular momentum, or when creating very heavy nuclei. Each forefront area reveals a unique competition between classical destabilizing forces and quantum mechanical binding forces. However, only when these diverse areas of research are considered together does a universal picture of nuclear hehavior evolve.

We are working in several of these areas at Yale. We have been studying the proton evaporation from hot nuclei and have just completed the development of a silicon calorimeter which should allow the study of changes in nuclear structure with excitation energy at unprecedented precision. With the Yale theory group, we have been istudying and modeling nuclei at high angular momentum in order to investigate the microscopic origin of collective motion. We have also been studying nuclear isomerism at high spin. Finally, we have commenced studies of very heavy nuclei, in order to search for a predicted, but as yet unobserved, region of oblate deformed nuclei in the actinides. All these projects are continuing, hoth at Yàle and at laboratories in the U.S., Canada, and England. 
A.W. Wright Nuclear Structure Laboratory

New Haven, Connecticut 06511

Title of Project: Nuclear Astrophysics

Persons in Charge: (A) P. D. Parker, (B) M. Gai

Tolephone: $\quad$ (203) $432-3090$

(A) The current focus of our nuclear astrophysics program is on the study of explosive nucleosynthesis which occurs on time scales of the order of seconds or less and which must therefore include nuclear reactions involving radioactive nuclei which cannot decay during the time scale characteristic of the explosion. Our studies involve the determination of the rates of those reactions through measurements of the energies and partial widths of the relevant resonances.

A recent example of these nuclear spectroscopy measurements includes our study of the transition from the HotCNO cycle to the rp-process (a process of rapid-proton capture through successively heavier nuclei to the Fe-Ni peak). This transition involves nuclear reactions leading from the ${ }^{14} \mathrm{O}$ and ${ }^{15} \mathrm{O}$ nuclei in the HotCNO cycle to ${ }^{20} \mathrm{Na}$ at the start of the rp-process and includes the following reactions ${ }^{14} \mathrm{O}(\alpha, p){ }^{17} \mathrm{~F},{ }^{17} \mathrm{~F}(\mathrm{p}, \gamma){ }^{18} \mathrm{Ne},{ }^{15} \mathrm{O}(\alpha, \gamma){ }^{19} \mathrm{Ne},{ }^{19} \mathrm{Ne}(\mathrm{p}, \gamma){ }^{20} \mathrm{Na}$, etc., whose compound nuclear resonances we have measured using complementary light and heavy-ion reactions such as ${ }^{16} \mathrm{O}\left({ }^{3} \mathrm{He}, \mathrm{n}\right){ }^{18} \mathrm{Ne},{ }^{20} \mathrm{Ne}(\mathrm{p}, \mathrm{t}){ }^{18} \mathrm{Ne},{ }^{12} \mathrm{C}\left({ }^{12} \mathrm{C},{ }^{6} \mathrm{He}\right){ }^{18} \mathrm{Ne},{ }^{19} \mathrm{~F}\left({ }^{3} \mathrm{He}, \mathrm{t}\right){ }^{19} \mathrm{Ne}(\alpha){ }^{15} \mathrm{O},{ }^{20} \mathrm{Ne}\left({ }^{3} \mathrm{He}, \mathrm{t}\right){ }^{20} \mathrm{Na},{ }^{12} \mathrm{C}\left({ }^{14} \mathrm{~N},{ }^{6} \mathrm{He}\right){ }^{20} \mathrm{Na}$, etc.

(B) Massive stars burn hydrogen to form helium and after a short period begin burning helium to form first carbon and then oxygen. The nucleosynthesis of carbon and oxygen serves as the doorway for producing the heavy elements found in the universe. The fusing of three alpha-particles to form ${ }^{12} \mathrm{C}$ is well understood, but the rate of formation of ${ }^{16} \mathrm{O}$ via the ${ }^{12} \mathrm{C}(\alpha, \gamma){ }^{16} \mathrm{O}$ reaction is still an open question. We are currently studying this fusion process by measuring its time-reversed process, the disintegration of ${ }^{16} \mathrm{O}^{\circ}$ into $\alpha+{ }^{12} \mathrm{C}$. In this experiment, the radioactive decay of ${ }^{16} \mathrm{~N}$ is used to form (with a branching ratio of $10^{-5}$ ) the excited ${ }^{16} \mathrm{O}$ nucleus whose decay into ${ }^{12} \mathrm{C}+\alpha$ we then measure to determine the rate of the ${ }^{12} \mathrm{C}(\alpha, \gamma)^{16} \mathrm{O}$ reaction. 


\section{YALE UNIVERSITY}

A.W. Wright Nuclear Structure Laboratory

New Haven, Connecticut 06511

Title of Project: Relativistic Heavy-Ion Collisions

Person in Charge: $\quad$ B. S. Kumar

Telephone: $\quad$ (203) $432-3090$

Scientific Staff: $\quad$ A. Chikanian, G. Diebold

The main goal in studies of relativistic heavy-ion collisions is the creation and investigation of hot and dense states of nuclear matter and quark matter. Several experiments have been underway both at the BNL AGS facility on Long Island, and CERN in Europe. Our own group has been actively involved in the program at BNL on several experiments, E814, E878, E886, E813, E864, and PHENIX. Of these, the first has been completed, and the next three have collected data. E864 is presently being reviewed for funding, and PHENIX is being designed and is expected to be ready to use heavy-ion beams from the new RHIC facility.

Our program has thus far focused on characterizing the systems created in heavy-ion collisions through studies of the spectra of the incident and produced particles. In particular, we have studied the distributions in rapidity and transverse momentum of protons, antiprotons, and light nuclei. Our studies have demonstrated that at energies of $15 \mathrm{GeV}$ per nucleon, one can indeed create states of . nuclear matter at high baryon density and temperatures. The conditions created are close to what have been predicted to be needed for the creation of novel states of matter such as quark matter. In particular, baryon-rich environments are expected to be conducive for the production of cold quark matter, or strangelets. In experiment E864, we intend to search, with unprecedented sensitivity, for such objects. We hope this will open up new avenues for the investigation of QCD, the fundamental theory of strong interactions. The PHENIX experiment proposes, with beams of much higher energy, to create similar matter at higher temperatures, the quark-gluon plasma. Such matter is believed to have existed in the early universe. For all of these experiments, we have constructed or will construct detector systems, and will develop schemes for the analysis of data. Our program involves the active participation of several undergraduate and graduate students. 
A.W. Wright Nuclear Structure Laboratory

New Haven, Connecticut 06511

\section{Title of Project: $\quad$ (A) APEX, (B) CEBAF}

Persons in Charge: J. S. Greenberg, C. J. Lister, M. Gai

Telephone:

(203) 432-3090, (203) 432-3090

\section{Sciontific Staff: $\quad$ A. Chishti, P. Chowdhury}

(A) Our discovery, in collaboration with the Gesellschaft fur Schwerionenforschung, Darmstadt (GSI) laboratory in Darmstadt, of mono-energetic pairs of electrons and positrons emitted when superheavy atomic systems are formed in collisions, has presented an interesting puzzle whose explanation may involve new fundamental phenomena, including the production of new objects whose decay could produce positrons with the characteristics observed. Recently, our attempts to form such an object directly by combining electrons and positrons at the mass suggested by the heavy-ion scattering experiments have met with negative results. The source appears to be associated with the heavy-ion complex, possibly through the exceptionally large electric fields generated in the collisions. Using the superior qualities of the uranium beam being produced at the Argonne Tandem Line Accelerator System at ANL and a new comprehensive spectrometer called Argonne Positron-Electron Experiment (APEX), we are presently pursuing new experiments in collaboration with groups from ANL and five other universities to further study the anomalous pair production in the superheavy atomic systems. These experiments have a number of advantages over those we carried out at GSI and should provide new information that could resolve the origin of the anomalous positron puzzle.

(B) The study of the structure of nuclei and, in particular, the study of broken reflection symmetry exhibited by enhanced electric dipole transitions and parity doublets in nuclei leads us to look at the structure of hadrons. We find that the excited nucleon (proton or neutron) as well as the excited delta exhibit broken reflection symmetry and parity doublets. We have proposed to measure electromagnetic properties of the nucleon at CEBAF, and 500 hours of beam time were awarded for this experiment to be performed with the $\mathrm{N}^{*}$ collaboration of Hall $B$ of CEBAF. 


\section{YALE UNIVERSITY}

Department of Physics

New Haven, Connecticut 06511

Title of Project: Nuclear Theory

Person in Charge: F. Iachello

Telophone: $\quad$ (203) 432-6944

Scientlfic Staff: $\quad$ Y. Alhassid, D. Kusnezov, Y. Devi, N. Manini

FY 1992 Funding: $\$ 394,000$

The research program of this group centers on the use of algebraic methods to describe physical phenomena.

The major effort is in the application of algebraic methods to nuclear structure physics. This group has developed a model, the interacting boson model, capable of describing properties of medium mass and heavy nuclei with an accuracy better than 90 percent. In recent years, this model has been extended to treat nuclei under extreme conditions, such as nuclei far from the line of stability and nuclei with large angular momenta. Another important aspect investigated in the last few years has been that of the chaotic properties of the model and its relevance to the study of chaos in quantum mechanical systems.

This group is now applying similar algebraic methods to the study of the structure of the nucleon in terms of quarks and gluons. This part of the program will be particularly relevant to the analysis of the experimental data which will be taken at the CEBAF facility presently under construction in Virginia.

Another line of research deals with the combination of algebraic methods for structure with algebraic methods for scattering. Using these methods, it has been possible to treat collisions between complex systems, such as those between heavy ions and between molecules. 
Physics Department

New Haven, Connecticut 06511

Title of Project: $\quad$ Measurements of Rare Composite Objects and Searches for New Forms of Matter in Relativistic Heavy-Ion Collisions

Person in Charge: J. Sandweiss

Telephone: $\quad$ (203) 432-3358

Scientific Staff: $\quad$ F. S. Rotondo, R. Majka, E. Wolin

FY 1992 Funding: $\$ 128,000$

The primary goal of our research program is the exploitation of the unique collision environment provided by the interactions of high energy ( $12 \mathrm{GeV} / \mathrm{c}$ per nucleon or greater) heavy-ion collisions to search for new forms or states of matter. A secondary but essential goal is to use the highly sensitive search experiments themselves to learn more about the nature of the collisions. The two goals are symbiotic because an understanding of the collision dynamics is necessary for evaluating the significance, and indeed for determining the requirements, of the search experiments.

The high energy heavy-ion collisions in the fixed-target geometry (at the BNL AGS, with gas jet target at RHIC, or in the fixed-target heavy-ion beam experiments at CERN) provide "large" volumes of hot, dense nuclear matter with high strangeness content and high baryon content. There is no other way of producing new systems with large strangeness in the laboratory. Several such high strangeness systems have been hypothesized. The best known are the strange quark matter systems (strangelets) which might in fact be the true ground state of hadronic matter. The discovery of strange quark matter would have major technological, astrophysical, and theoretical consequences.

As noted above, our experiments study, with high sensitivity, a number of interesting processes known to occur in the collisions. Among these are the production of various light nuclei produced by coalescence out of the fireball of hadrons created in central heavy-ion collisions. Another process of considerable interest is the production of antimatter in the collisions. The high sensitivity of these experiments is needed for significant studies of these topics.

In the past, our research program has made use of an existing experiment at AGS (E814). First, searches have been carried out and published using toe E814 apparatus. We are now in the construction phase of a major new experiment for the i:US E864, which will improve the sensitivity and experimental reach by many orders of magnitude over E814. The ultimate sensitivity will be a few times.

Finally, we are studying a future experiment using a gas jet target at RHIC, and reusing much of the E-864 apparatus, which would further extend the reach of these studies. 
Department of Physics

New Haven, Connecticut 06520

Title of Project: Medium Energy Elementary Particle Physics

Person in Chargo: V. W. Hughes

Telephone: $\quad$ (203) 432-3819

Scientific Staff: $\quad$ M. Boutemeur, D. Ciskowski, S. Dhawan, X. Fei, M. Janousch, W. Schwarz

FY 1992 Funding: $\quad \$ 646,000$

There are two project objectives: (1) Precision studies of the simple muonium atom $\left(\mu^{+} e^{-}\right)$to test the fundamental theory of quantum electrodynamics and the unified electroweak theory - two aspects of the current standard theory of particle physics. In addition, our experiments measure fundamental properties of the muon-its mass and magnetic moment-and the fine structure constant $\alpha$. It is generally agreed that the present standard theory is incomplete, so it is important to test it sensitively to determine its limits and to look for clues to a better understanding.

During the past 3 years at LAMPF, we have completed a measurement of the Lamb shift and fine structure interval in the excited $n=2$ state of muonium and also the most sensitive search to date for the spontaneous conversion of muonium to antimuonium, a muon-number violating process which is not allowed in the standard theory. This latter result places a limit on the left-right symmetric theory which is a proposed extension of the standard theory.

At present, we are starting an experiment at LAMPF to measure the hyperfine structure interval and Zeeman effect in the ground state of muonium to ultrahigh precision and thus determine $\mu_{\mu}$ and $m_{\mu}$ to 50 parts per billion and the fine structure constant $\alpha$ to $25 \mathrm{ppb}$.

(2) Measurement of the spin-dependent structure functions of the proton and neutron and hence test important sum rules for the neutron and proton separately and also the Bjorken polarization sum rule which is fundamentally based on the assumptions of QCD.

A major experiment is underway at CERN-NA47 (with V.W. Hughes as spokesman) to determine the spin-dependent structure functions of the proton and neutron via measurement of the spin-dependent asymmetries in the scattering of high energy (100-200 GeV) polarized muons by polarized protons and deuterons. Experimental checkout and initial data-taking took place in 1991, and further data-taking occurred for a six-month run in 1992 with a polarized deuteron target.

A major anomaly was discovered several years ago (with Yale participation) in a similar experiment at CERN with a polarized proton target. A polarization sum rule for the proton was violated with the implication that the quark spins do not contribute to the proton spin. An enormous explosion of theoretical papers followed. Our new CERN experiment should contribute importantly to extension of our knowledge and clarification of our understanding of the internal spin structure of the nucleon. The entire field of experiments to measure spin-dependent structure functions was initiated by our Yale group in experiments at SLAC in the 1970s. 


\section{Glossary of Nuclear Physics Terminology}


A

Accolerator

AGS

Alpha Particle

Amu

ANL

Antimatter

Antiparticle

Atom

Baryon

Beta Decay
The atomic mass number of a nucleus. $A=N+Z$, where $N$ is the number of neutrons and $\mathrm{Z}$ is the number of protons.

A device which increases the speed and energy of nuclear particles, and is a source of beams of nuclei for nuclear physics experiments.

Alternating Gradient Synchrotron. A circular accelerator at Brookhaven National Laboratory which accelerates nuclei to high energies.

The nucleus of the Helium atom with $A=4$.

Atomic mass unit, defined as $1 / 12$ of the mass of an atom of Carbon with $\mathrm{A}=$ 12.

Argonne National Laboratory, located southwest of Chicago, Illinois.

Matter composed of antiparticles.

The partner of each fundamental particle, but with charge-like properties opposite to the particles. Particles and their antiparticles can annihilate to produce gamma-ray energy.

The smallest unit of a chemical element, consisting of a nucleus surrounded by electrons.

Any strongly-interacting particle composed of three quarks, including the proton and neutron.

The manifestation of the weak interaction in nuclei, in which a neutron converts into a proton, or vice versa.

Bismuth germanate, a scintillator material used to detect gamma rays. 
BNL

CEBAF

CERN

Cross Section

Delta

Deuteron

Electromagnetic

Force

Electron

Electron Volt (eV)

Electroweak Force

Equation of State
Brookhaven National Laboratory, located on Long Island, New York.

Continuous Electron Beam Accelerator Facility, located at Newport News, Virginia, under construction.

(Originally Centre European pour Recherche Nucleaire, now European Laboratory for Particle Physics.) A nuclear and high-energy physics facility located near Geneva, Switzerland.

The effective collision area for the interaction between a nucleus and a projectile, measuring the probability of a reaction.

The first excited state of the nucleon.

The simplest composite nucleus, consisting of a neutron bound to a proton.

The long-range force associated with electricity and magnetism, of intermediate strength between weak and strong force. The photon propagates the electromagnetic force.

An elementary particle much lighter than a nucleon. Electrons surrounding a nucleus determine the atom's chemical properties.

An energy unit equal to the energy gained by an electron accelerated by a potential difference of 1 Volt.

The combination of the electromagnetic and weak forces that unifies their description.

The description of how a material's properties depend on temperature and pressure.

A state of a system obtained by adding energy to its lowest (ground) state. 
Fm (Formi)

Fission

FNAL

Form Factor

Fusion

Gamma Ray

GeV

Gluon

Gravitation

Ground State

GSI

Hadron

Heavy lon
Abbreviation for femtometer, $10^{-15}$ meter, which is about the diameter of a nucleon.

The splitting of a nucleus into two or more pieces.

Fermi National Accelerator Laboratory, a high energy physics laboratory, located at Batavia, Illinois.

A factor in collision equations that summarizes the internal properties of a particle.

The joining of two nuclei to make a heavier nucleus.

An energetic photon emitted in the decay of excited states and unstable particles.

An energy unit equal to $10^{9} \mathrm{eV}$, formerly called $\mathrm{BeV}$ (billion electron volts).

The particle that propagates the force between quarks, but which is probably not observable in isolation.

The weakest of the four fundamental forces, giving weight to matter and governing astronomical motions.

The lowest energy state of a system.

A heavy-ion accelerator laboratory near Darmstadt, West Germany.

Any particle interacting by the strong force, such as mesons, nucleons, and quarks.

An atomic nucleus containing more than four nucleons. 
Hypernucleus

Isospin

Isotopes

Kaon

Lambda

LAMPF

LANL

LBL

Lepton

Meson

Mov

Muon
A nucleus in which one or more nucleons is replaced by a hadron with nonzero strangeness, such as a lambda.

A quantum number which distinguishes different charge states of the same particle. For example, the neutron and proton are (approximately) different isospin states of the nucleon.

Nuclei with the different neutron numbers but the same proton number, so that their nuclear properties differ but their chemical properties are nearly the same.

A meson with mass between that of the pion and nucleon, containing a strange quark.

A strange hadron which decays by the weak force into a nucleon and a pion.

Clinton P. Anderson Meson Physics Facility (also known as Los Alamos Meson Physics! Facility), located at Los Alamos, New Mexico.

Los Alamos National Laboratory, located at Los Alamos, New Mexico.

Lawrence Berkeley Laboratory, located in Berkeley, California.

One of the following particles neutrinos, electrons, muons, and tau particles, and their antiparticles. They interact by the electroweak force.

Any combination of quark and antiquark, for example, the pion.

An energy unit, equal to $10^{6} \mathrm{eV}$.

A lepton having properties similar to the electron but about 200 times more massive.

The number of neutrons in a nucleus. 
Neutrinos

Noutren

Nuclear Mattor

Nucleon

Nuclous

ORNL

Party

Paull Principlo

Photon

Picosecond

Plon

Polarization

Postron
Neutral particles interacting through the weak force and occurring in beta decay.

neutral baryon interacting mainly by the strong force and forming a major constituent of nuclei.

Matter composed mainly of nucleons.

A proton or neutron.

The central core of an atom, composed mainly of nucleons.

Jak Ridge National Laboratory, located in Oak Ridge, Tennessee.

Ine behavior of a system under mirror reflection is its parity. Systems interacting by strong and electromagnetic forces have mirror symmetry (conserve parity), but weak forces do. not conserve parity.

A rule that restricts the quantum numbers of 'Jentical particles. Because of it the properties of nuclei and atoms depend strongly on the number of nucleons and electrons.

Electromagnetic radiation, such as gamma rays, $\mathrm{X}$ rays, light, microwaves, and radio waves. Photons propagates the electromagnetic force.

A time unit equal to $10^{-12}$ second.

A strongly-interacting meson consisting of a quark-antiquark pair. Often used to describe the long-range part of the nuclear force.

The preferred orientation of the spins of an assembly of nuclei or atoms.

The positively-charged antiparticle of the electron. 
Proton

QCD

Quantum

Mechanics

Quantum Number

Quarks

Resonance

RHIC

Shell Model

Spectrometer
A charged baryon, the nucleus of the hydrogen atom, interacting by the strong and electromagnetic forces, and forming a major constituent of nuclei.

Quantum Chromodynamics. A theory of the interactions between quarks and gluons which may form the basis for the description of nuclear force properties.

A mathematical framework for describing interactions at the molecular level and smaller. At these levels energy exchanges between particles occur in discrete units called quanta.

A number representing a discrete property of a quantum mechanical system. Quantum numbers usually combine by simple rules. Examples are electric charge, spin, parity, strangeness.

Elementary particles interacting through exchange of gluons. They compose mesons, nucleons, and other baryons, but they probably cannot appear as free particles.

Rolativistic Particle A particle moving at a speed close to that of light. Under this condition it should be described by the theory of relativity.

A transient state of a nucleus, often observed as a peak in the cross section of a reaction as a function of energy, associated with the formation of a fairly stable intermediate state of a collision.

Relativistic Heavy Ion Collider, a colliding-beam accelerator facility under construction at Brookhaven National Laboratory. At RHIC the projectile and target nuclei are both accelerated to near the speed of light before they collide.

A theory of nuclear structure in which each nucleon is treated as moving under the average influence of the others, resulting in shell-like structures.

A device which spreads out the energies or momenta of particles so that they can be separately measured. 
Spin The angular momentum of a particle, related to its behavior under rotation of the observer.

Strangeness

A quantum number of elementary particles. Most hadrons in common nuclear matter have strangeness of zero.

Strong Force

The strongest of the fundamental forces. It provides most of the binding of nuclei.

Structure Function A measure of the response of a system to forces exerted by particles. Usually expressed in terms of their energy and the impulse given to the system.

Superconductor

A material with almost negligible resistance to the conduction of electricity. Usually a material at temperatures near absolute zero.

Symmetry

Regularity in the appearance of a system when it is described from an alternate viewpoint. Examples are parity (mirror description), spin (rotated reference frame), or time-reversal (time reflection).

Temperature

A measure of the random internal kinetic energy of a system. In nuclei, temperatures are usually indicated in $\mathrm{MeV}$, where the unit of $1 \mathrm{MeV}=12$ billion degrees Celsius.

Time Reversal

The symmetry property of a system relating to how it would appear if time could be run backwards.

TPC

Time Projection Chamber. A detection system using electric fields and the collection of charge as a function of time to measure the trajectories and characteristics of charged particles.

Trtium A hydrogen atom with two neutrons and a proton in its nucleus.

Triton The nucleus of tritium. 


\section{GLOSSARY}

Vortox An interaction region from which observed particles emerge.

Virtual Particle

W Boson

Weak Force

$\mathbf{Z}$

Z Boson
A particle which is created then annihilated during the course of an interaction.

A fundamental particle that mediates the weak interaction.

The fundamental force that describes, for example, nuclear beta decay. Its strength is between that of electromagnetism and gravity.

The number of protons in a nucleus. Also equal to the number of electrons in the neutral atom.

A fundamental particle mediating the weak interaction. 


\section{Index of \\ Investigators}




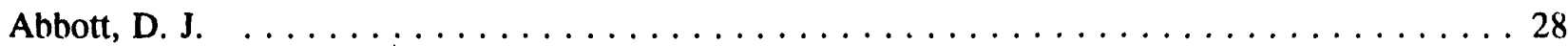

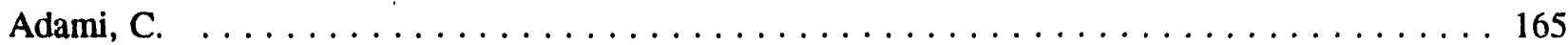

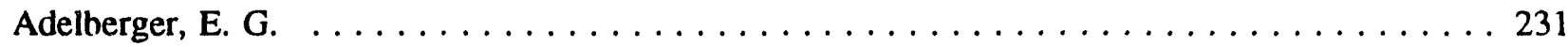

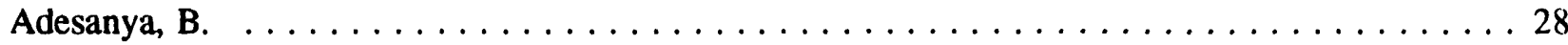

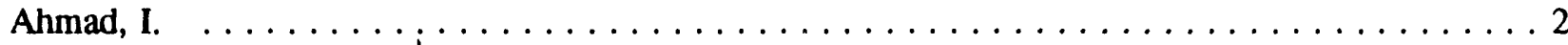

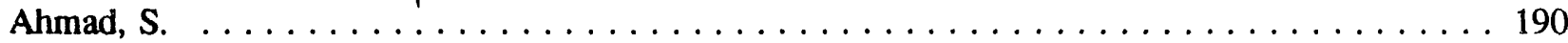

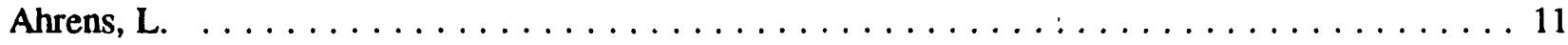

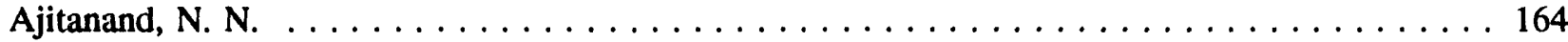

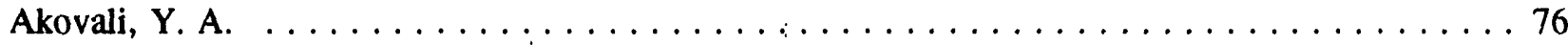

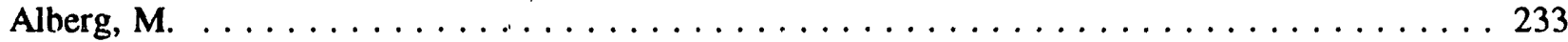

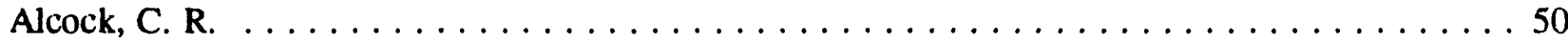

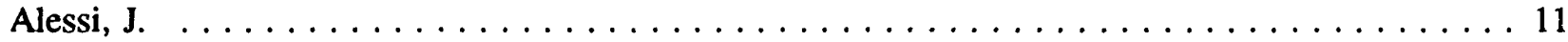

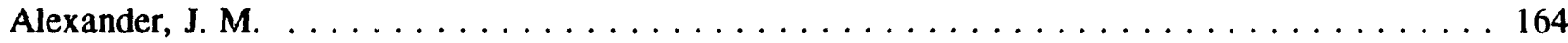

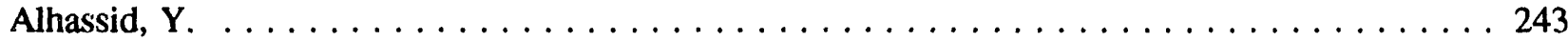

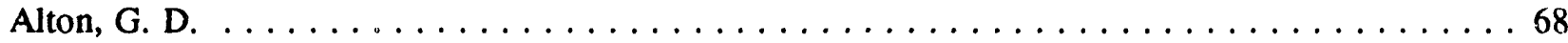

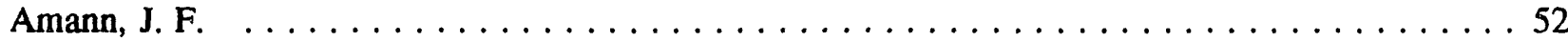

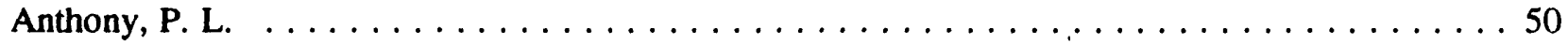

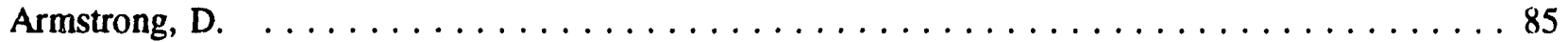

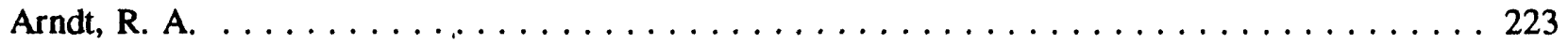

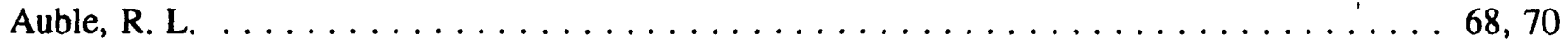

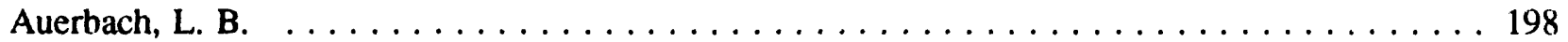

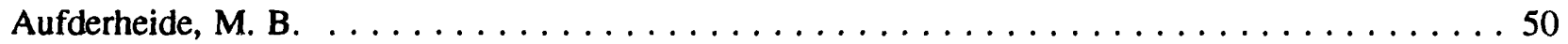

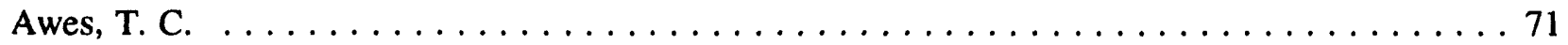

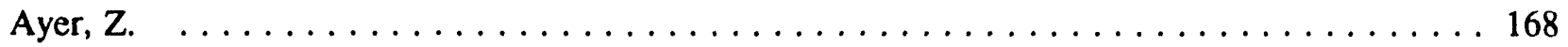

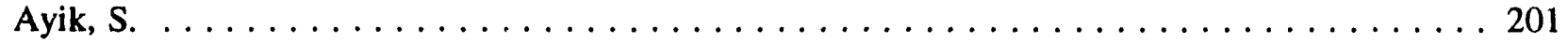

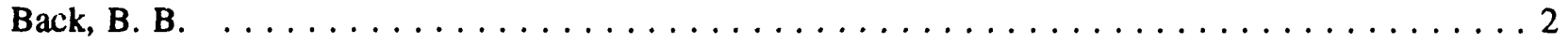

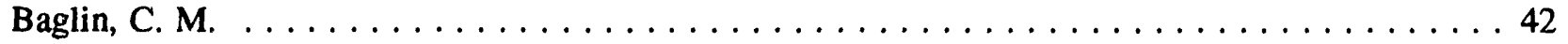

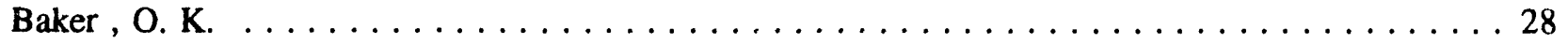

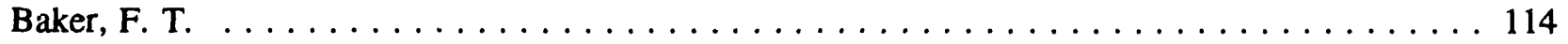

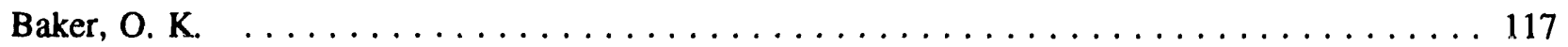

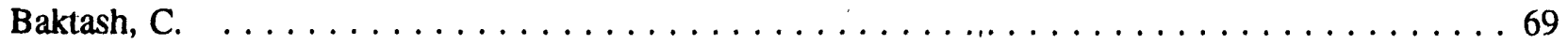

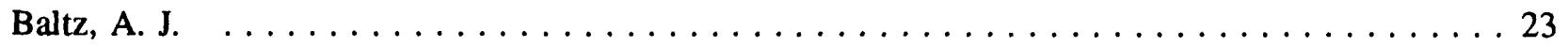

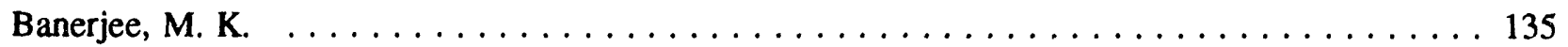

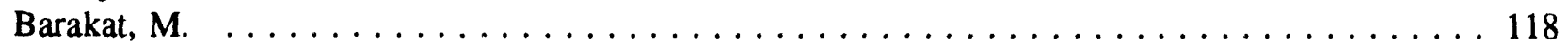

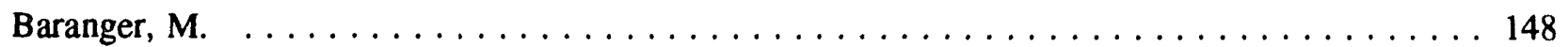

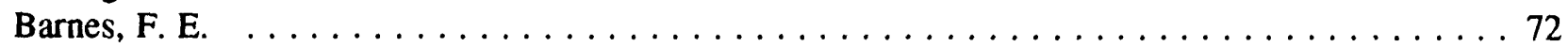

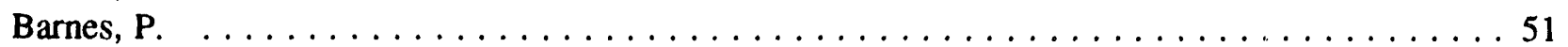

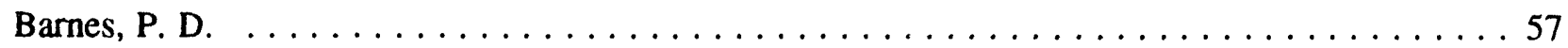

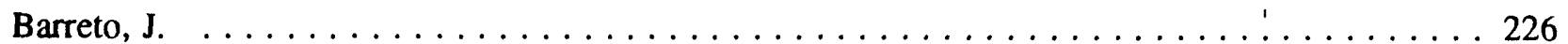

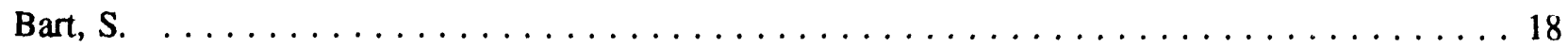

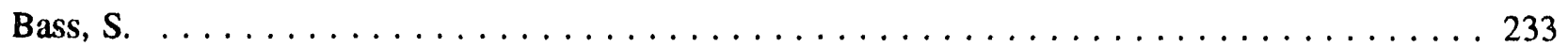

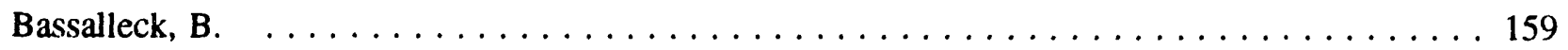

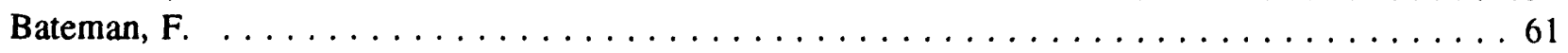

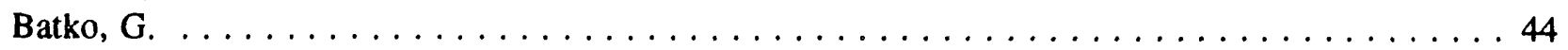

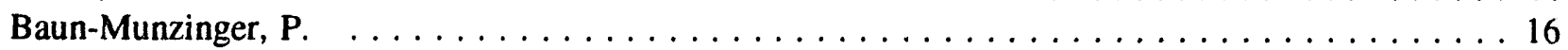

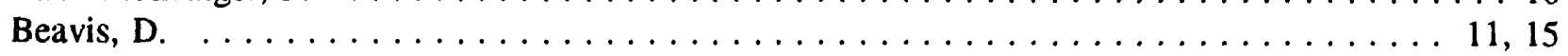

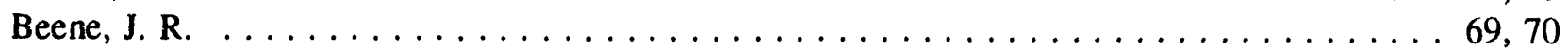

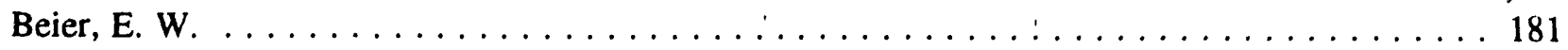

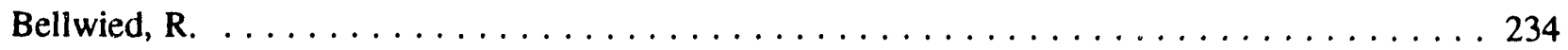




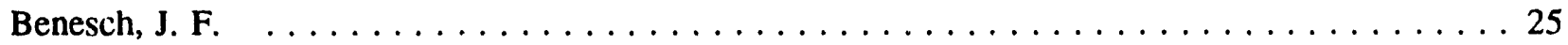

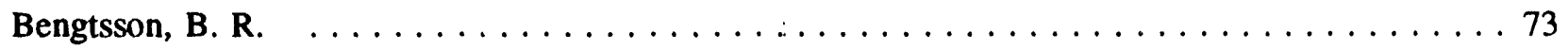

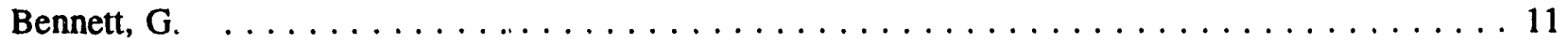

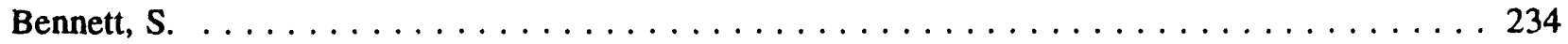

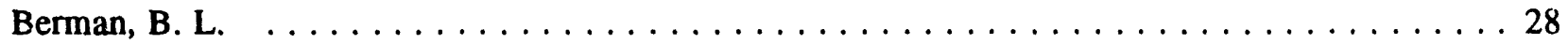

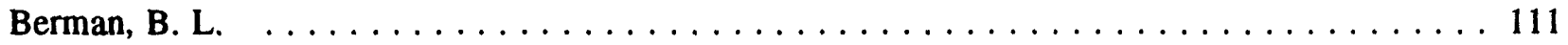

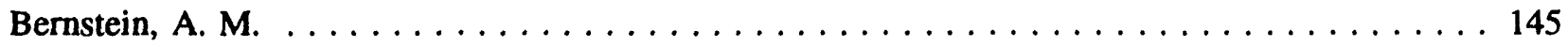

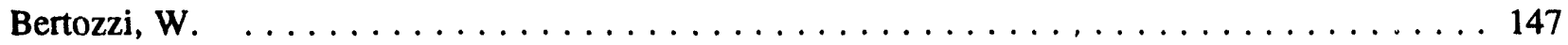

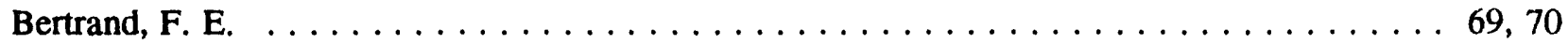

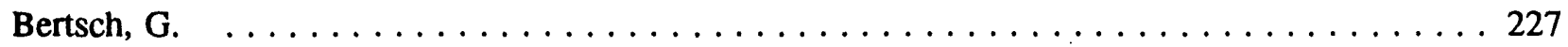

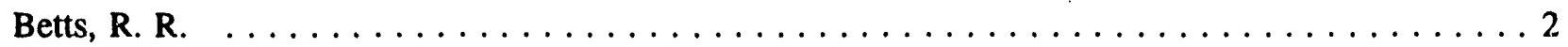

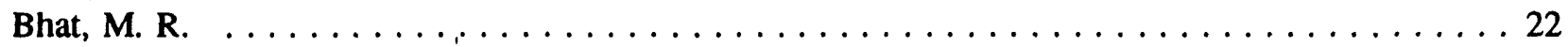

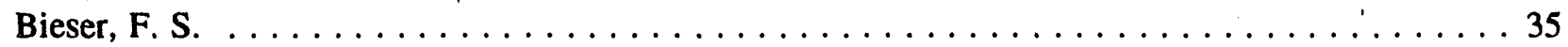

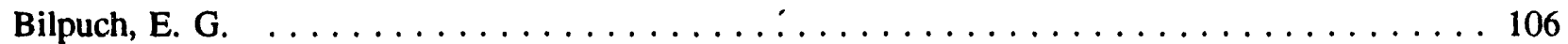

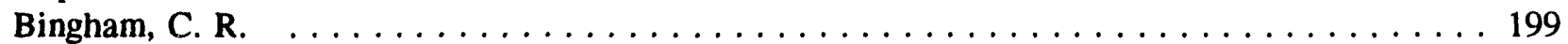

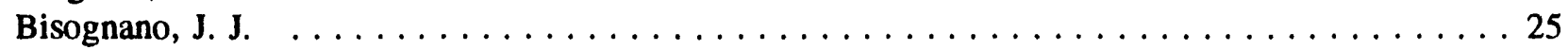

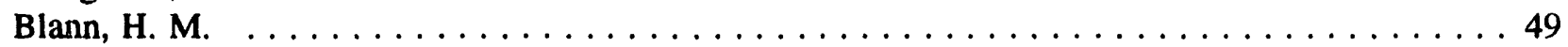

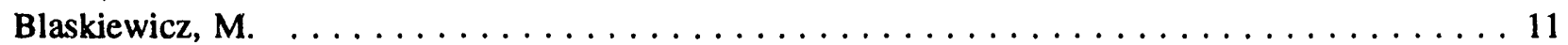

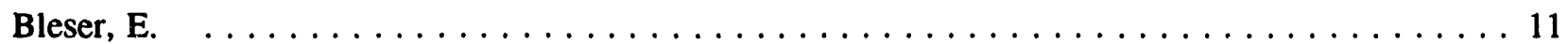

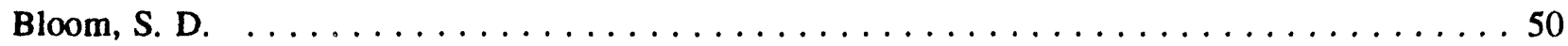

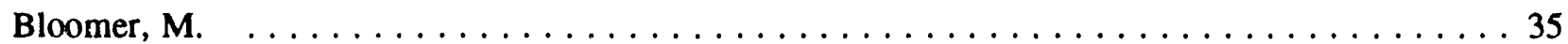

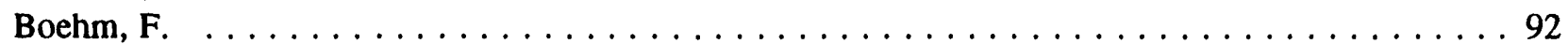

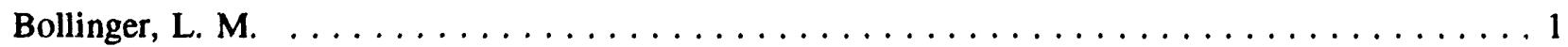

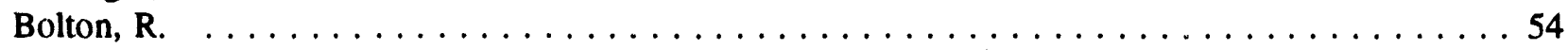

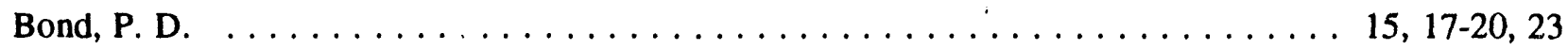

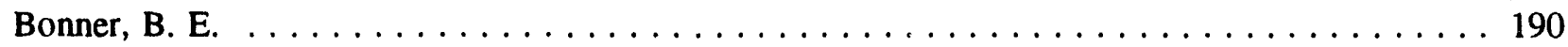

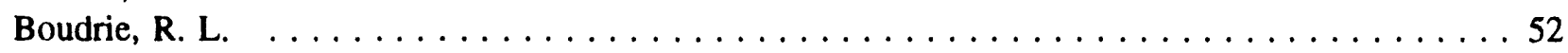

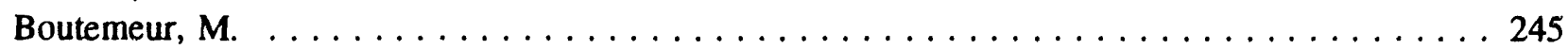

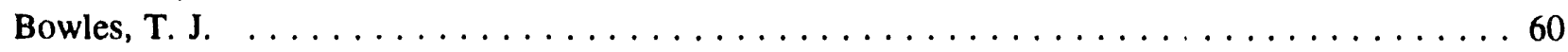

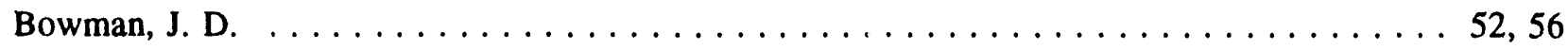

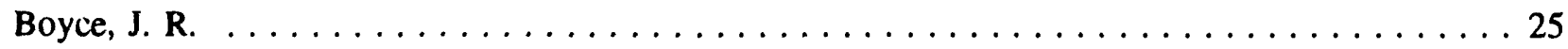

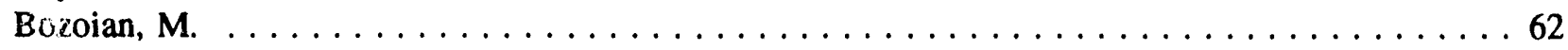

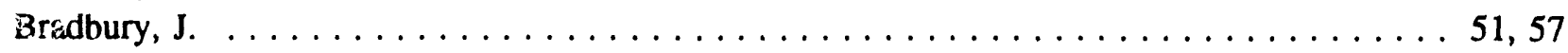

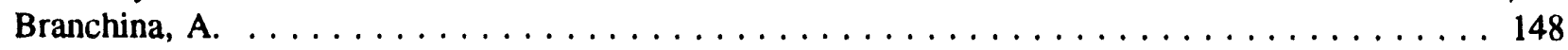

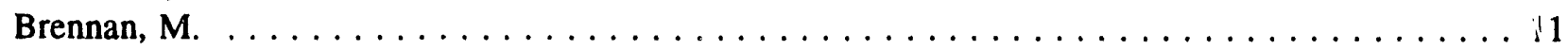

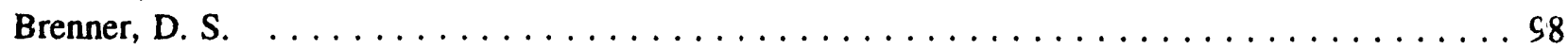

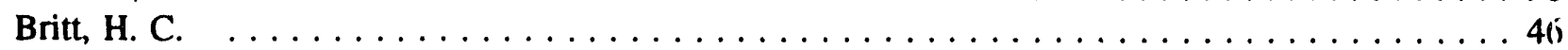

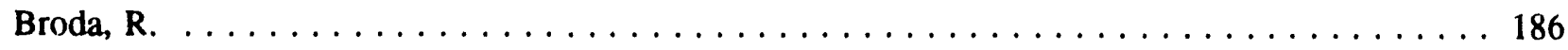

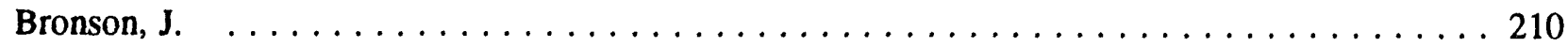

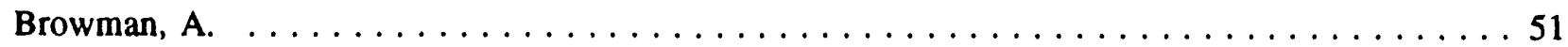

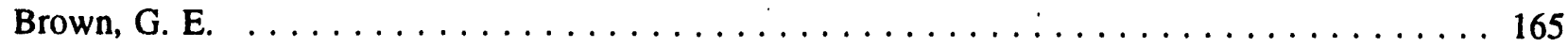

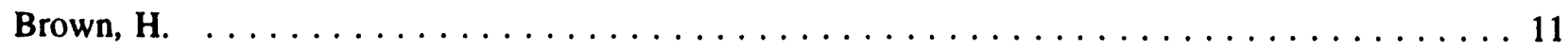

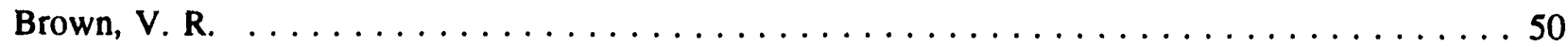

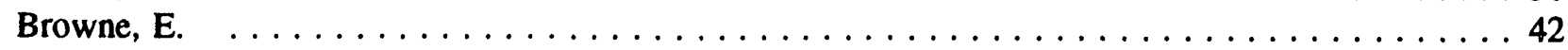

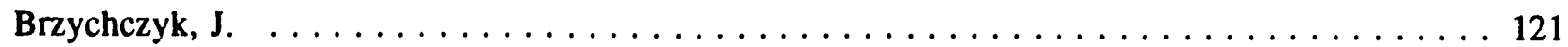

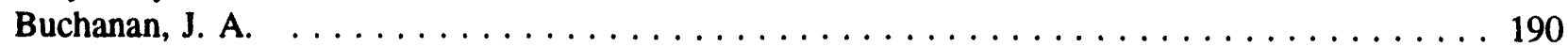

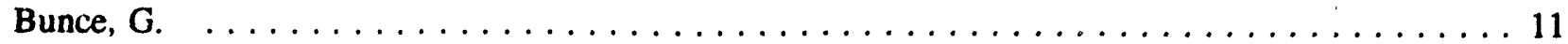

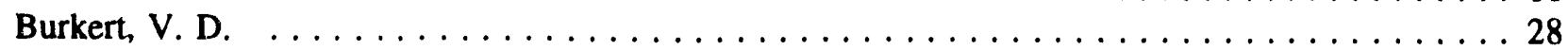

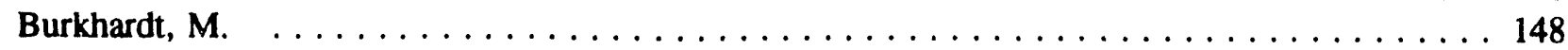

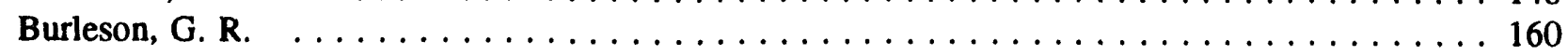




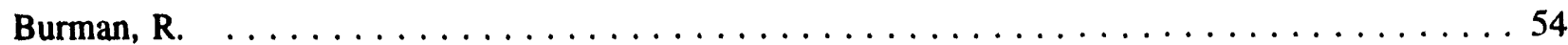

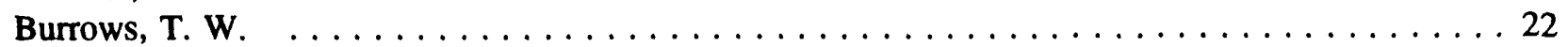

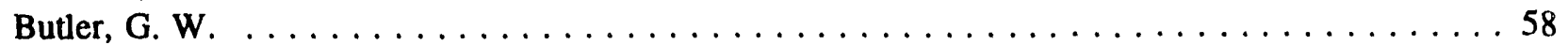

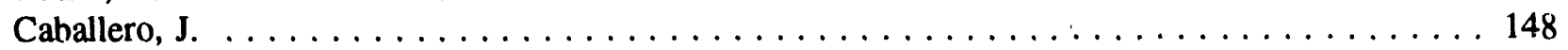

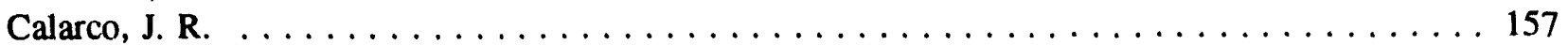

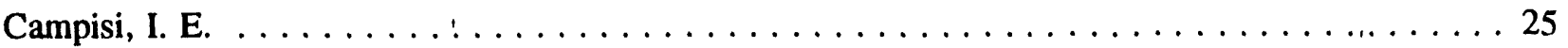

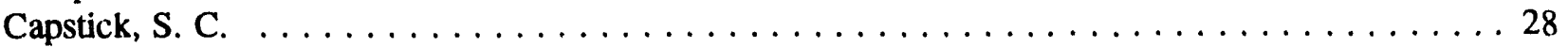

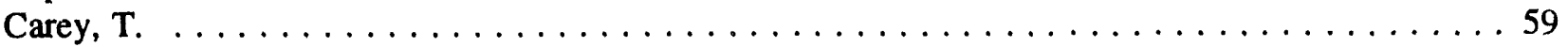

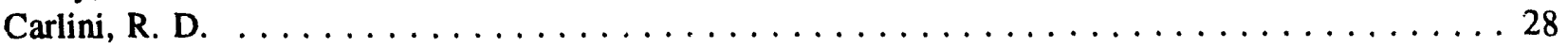

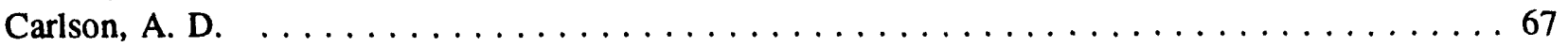

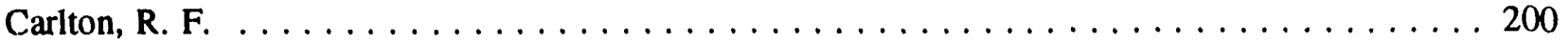

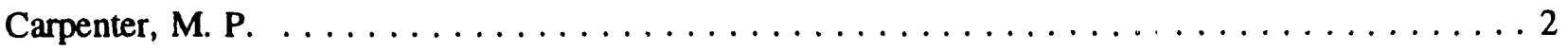

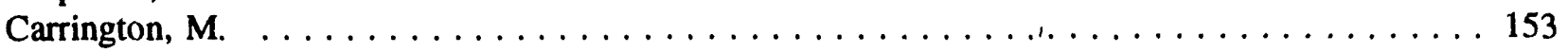

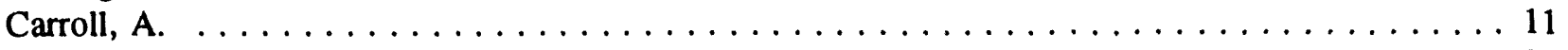

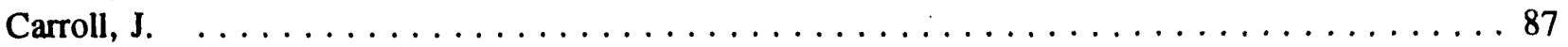

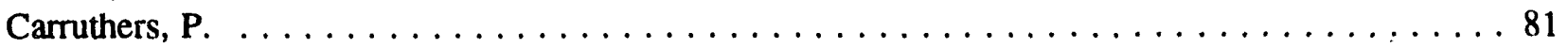

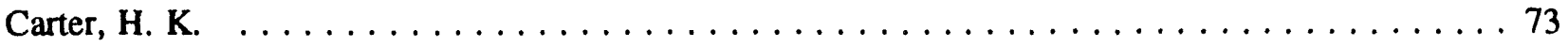

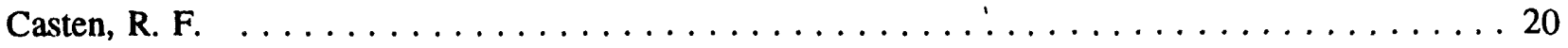

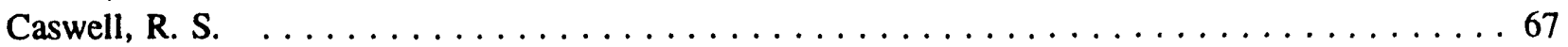

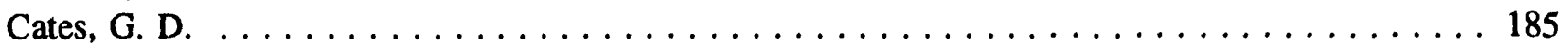

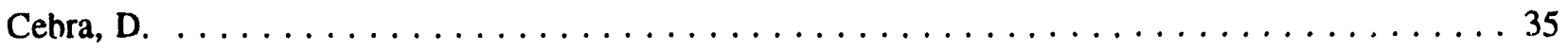

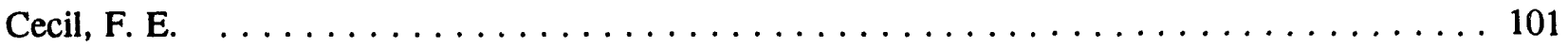

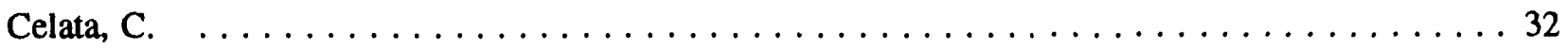

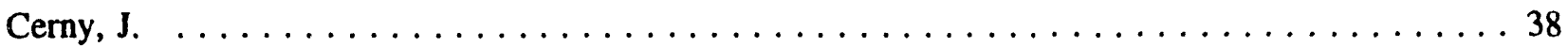

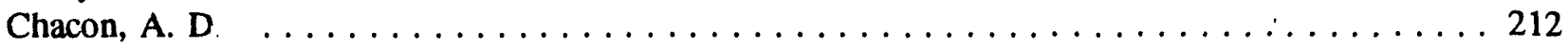

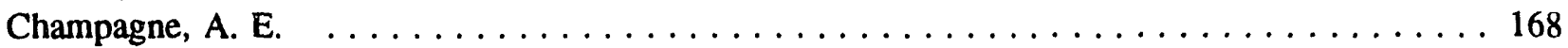

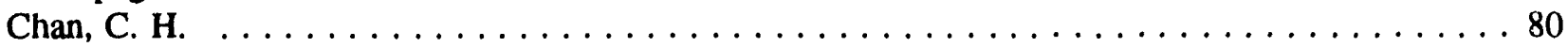

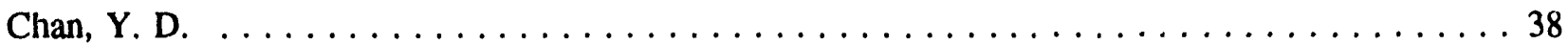

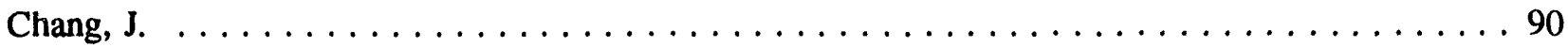

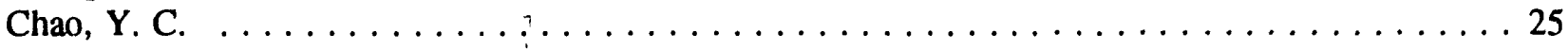

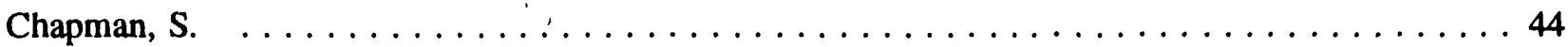

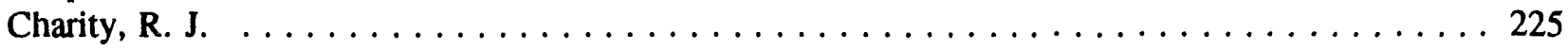

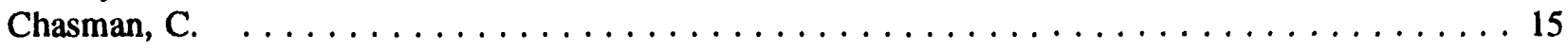

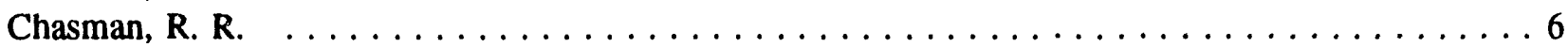

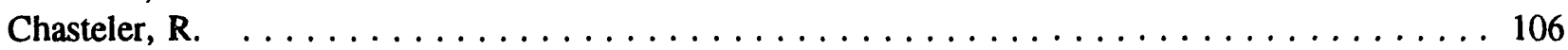

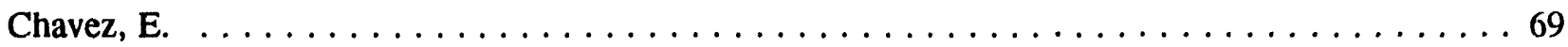

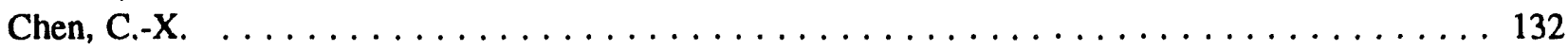

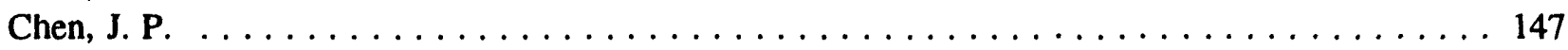

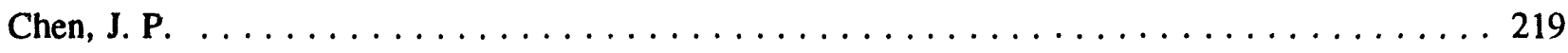

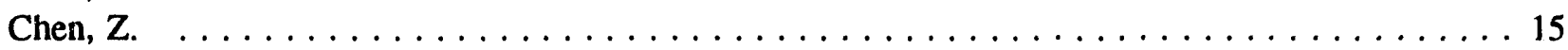

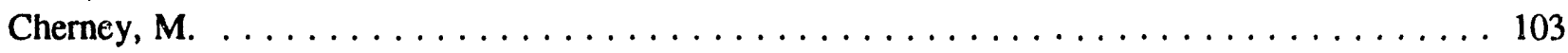

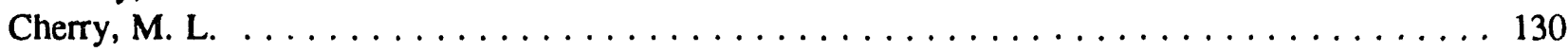

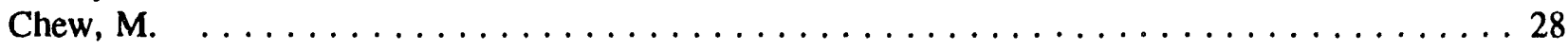

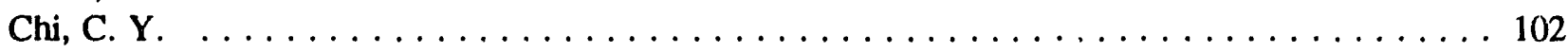

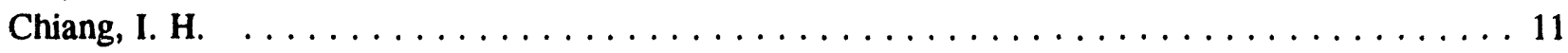

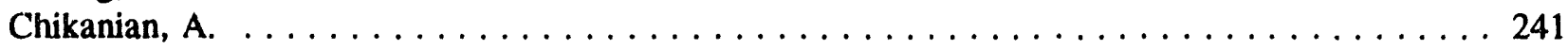

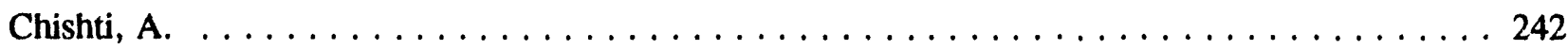

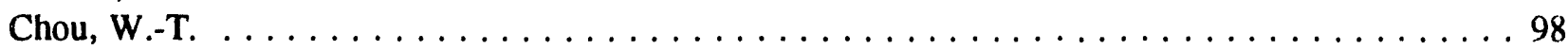

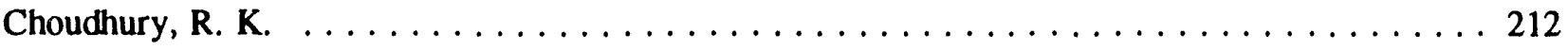


Cho'vdhury, P.

Chrien, R. E.

Christie, W.

Christodoulou, $M$.

Chu, S.-Y.

$\mathrm{Chu}, \mathrm{W}$.

Chu, Y. Y.

Ciskowski, D.

Clajus, $\mathrm{M}$.

Clark, D. J.

Clark, M. W.

Clegg, T. B.

Cleland, W. E.

Clement, J. M.

Coester, F.

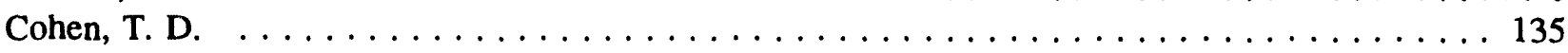

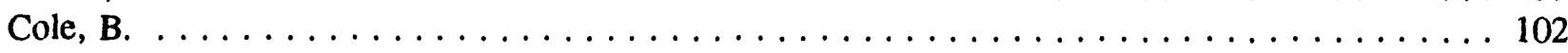

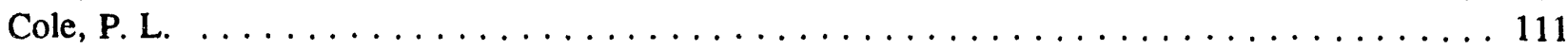

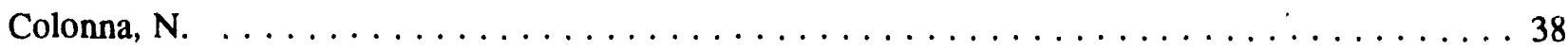

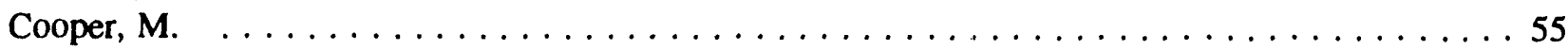

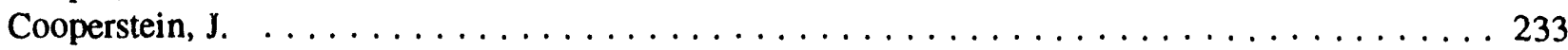

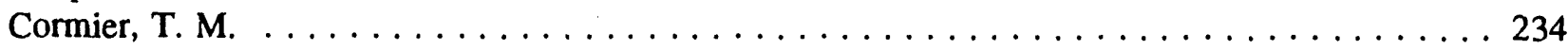

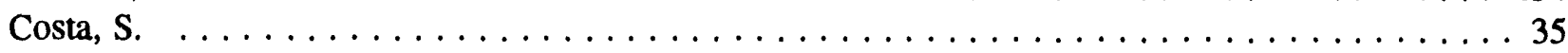

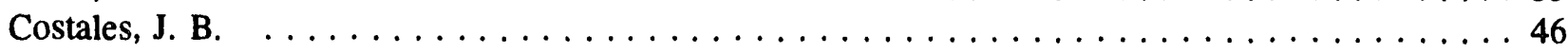

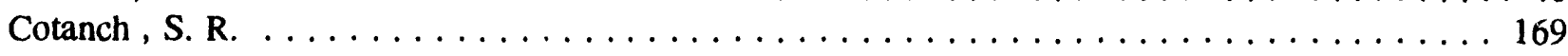

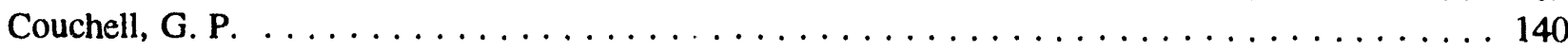

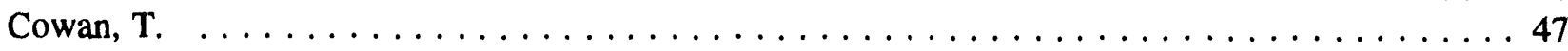

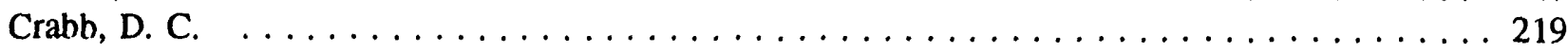

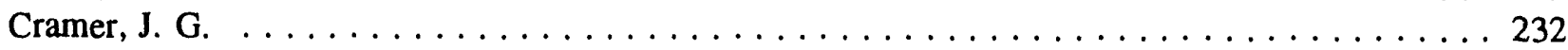

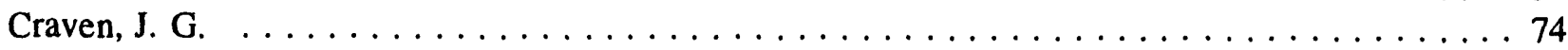

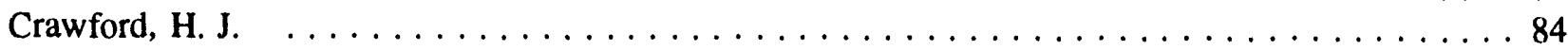

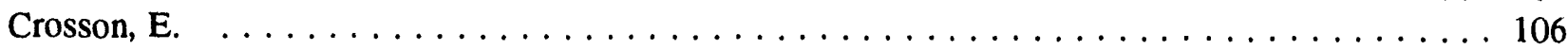

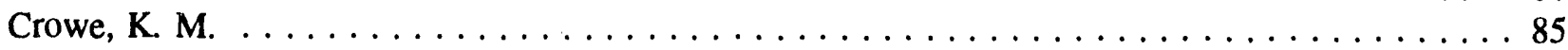

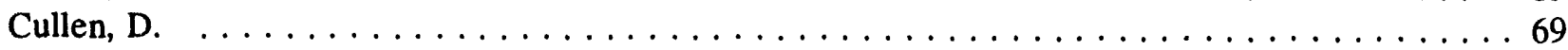

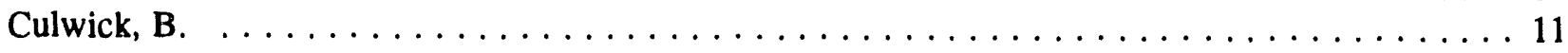

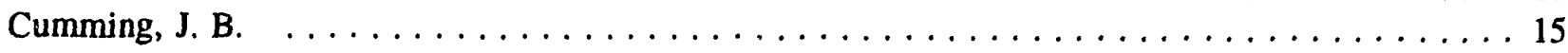

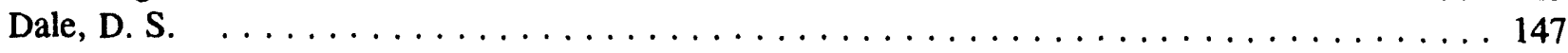

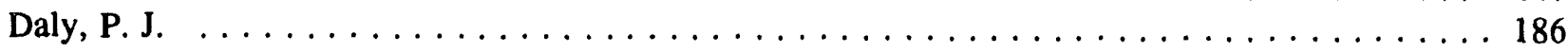

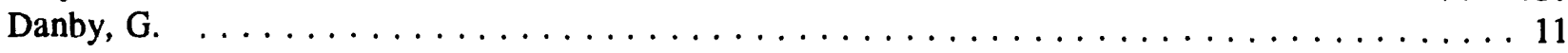

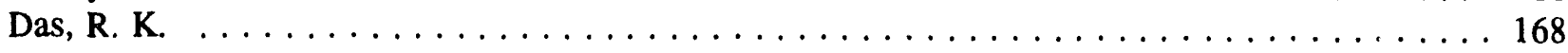

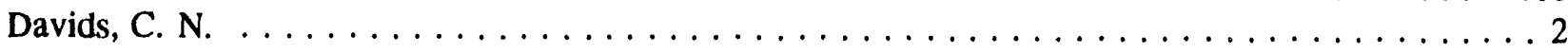

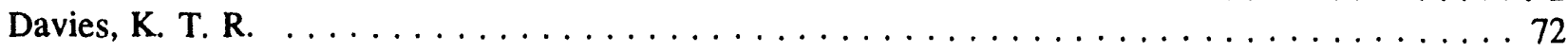

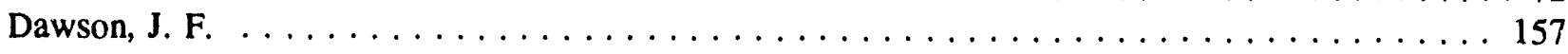

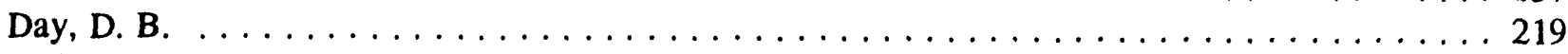

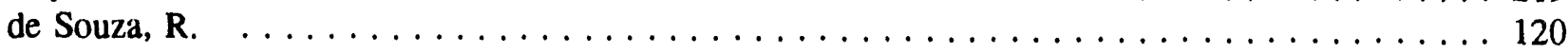

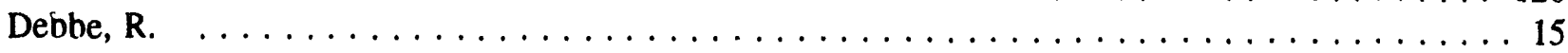

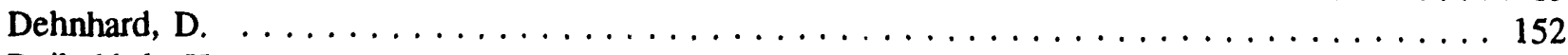

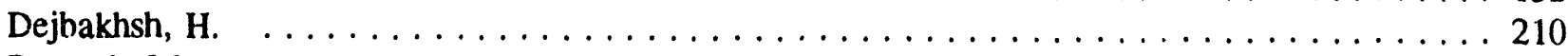

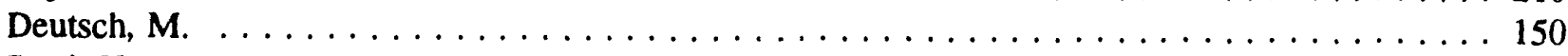

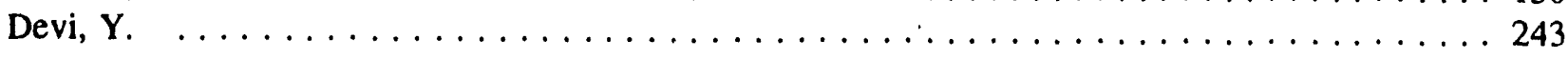




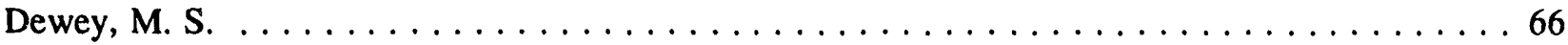

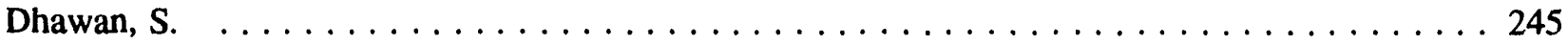

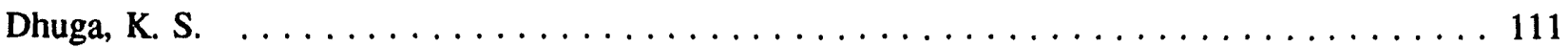

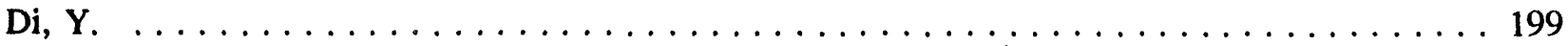

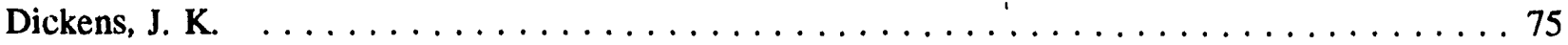

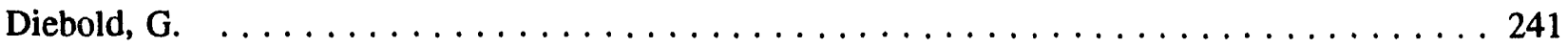

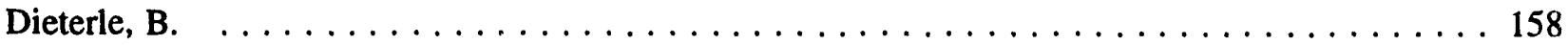

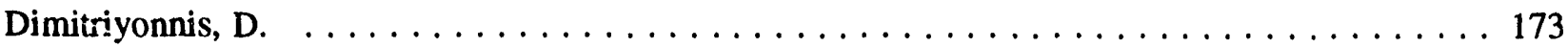

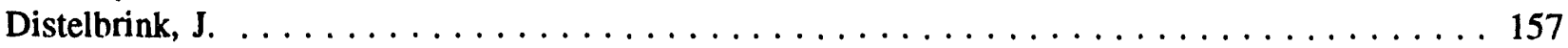

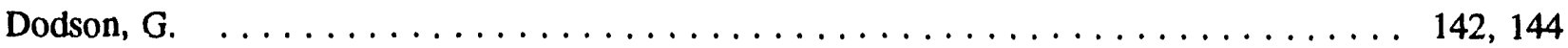

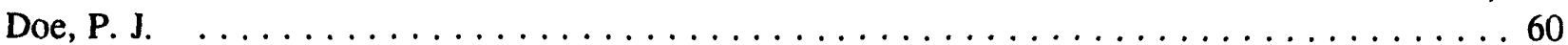

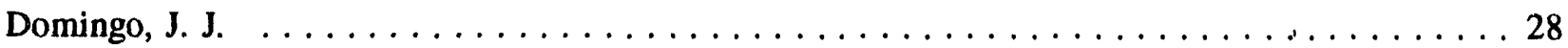

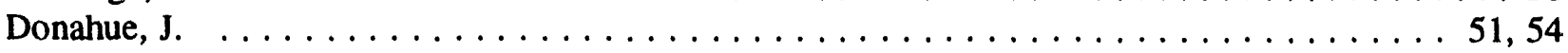

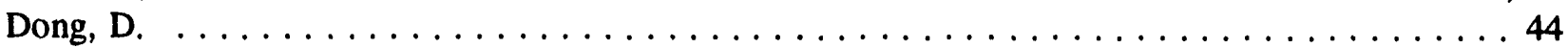

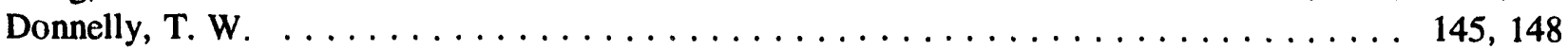

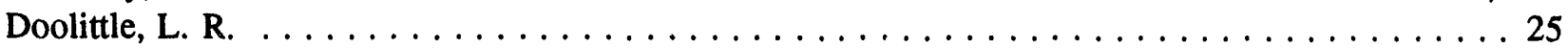

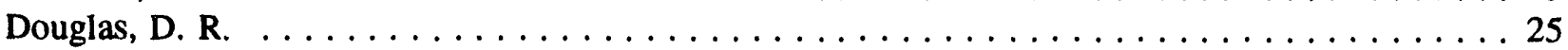

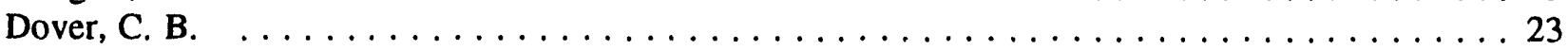

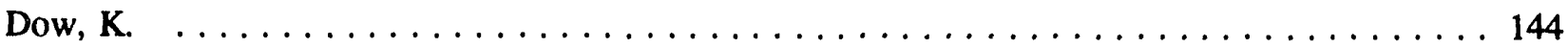

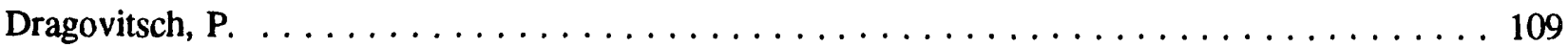

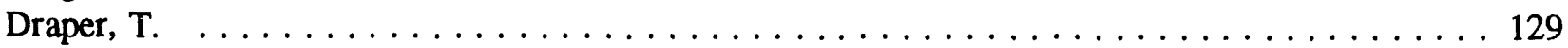

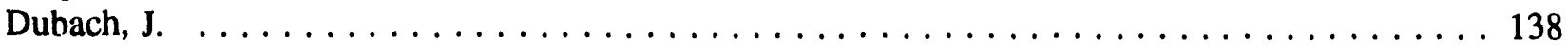

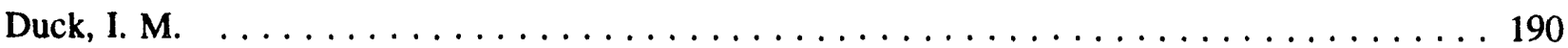

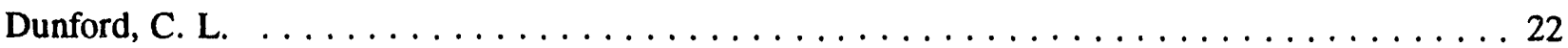

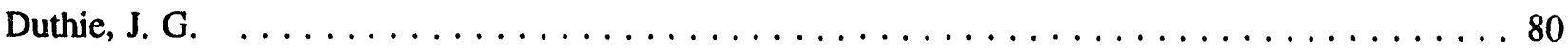

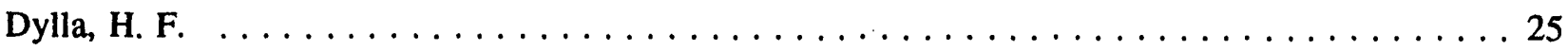

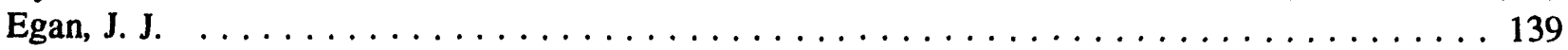

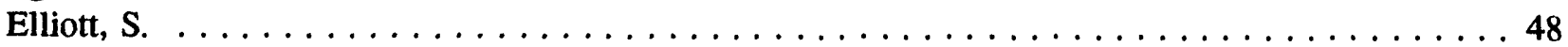

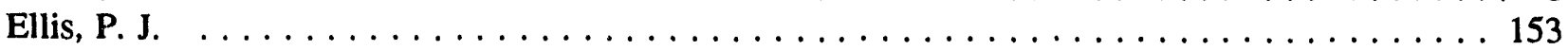

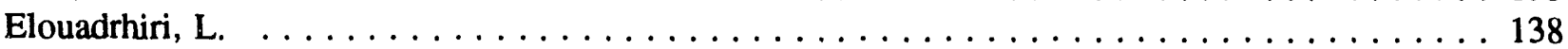

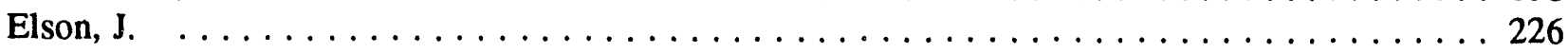

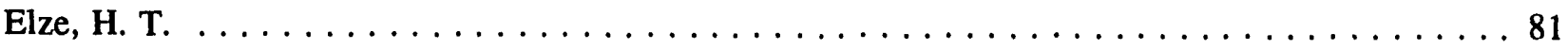

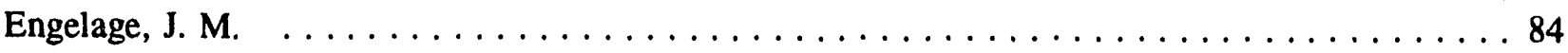

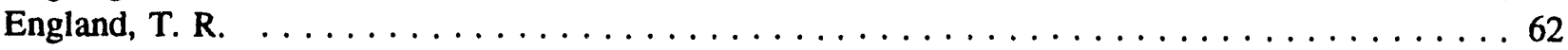

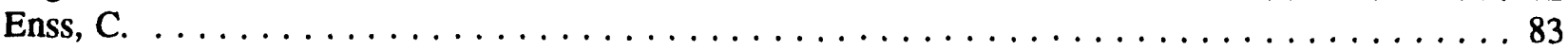

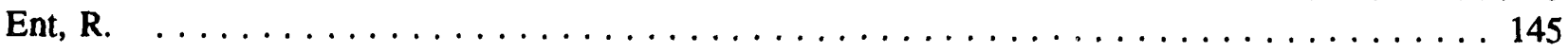

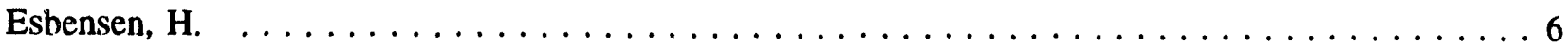

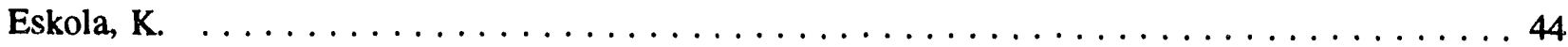

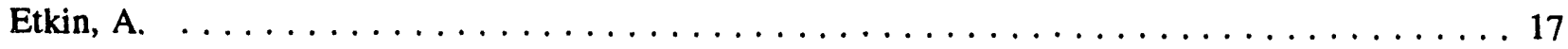

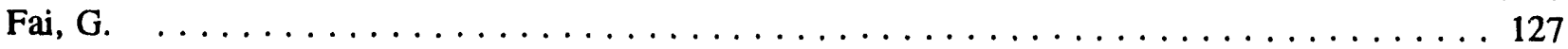

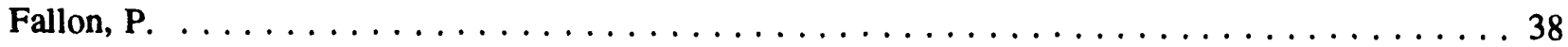

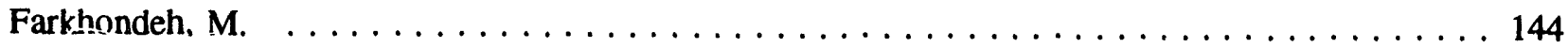

Ferlerspiel, F. J. $\ldots \ldots \ldots \ldots \ldots \ldots \ldots \ldots \ldots \ldots \ldots \ldots \ldots \ldots \ldots \ldots \ldots \ldots \ldots \ldots$

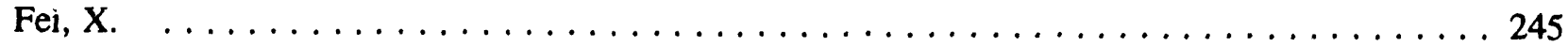

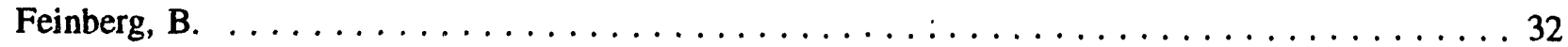

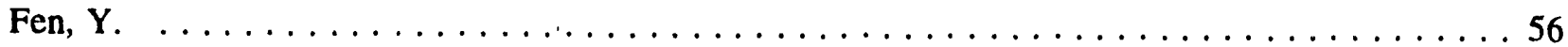

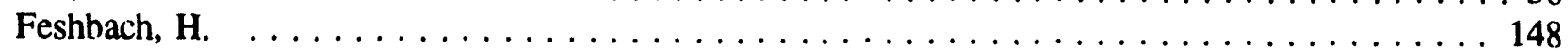

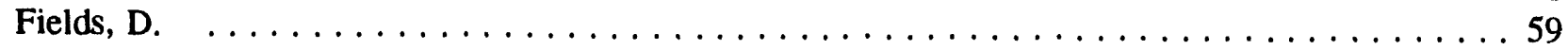

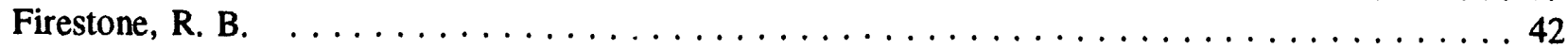




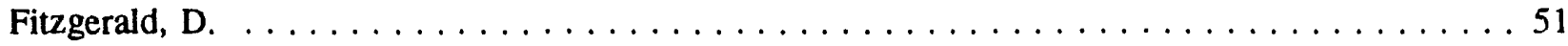

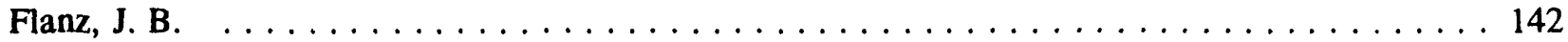

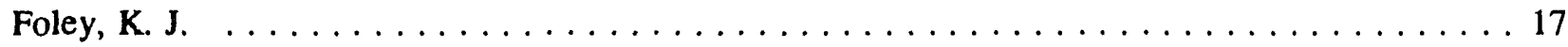

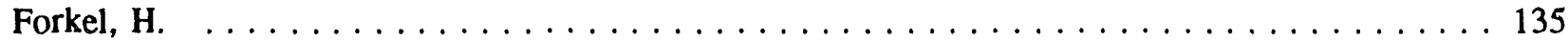

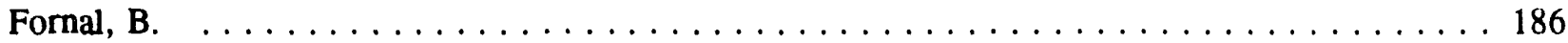

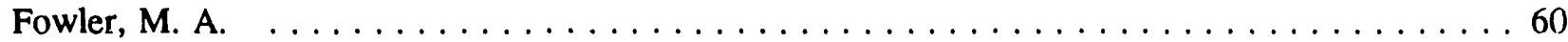

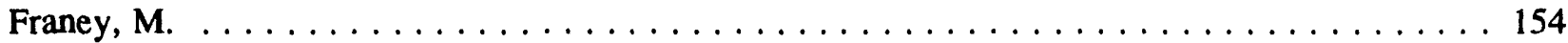

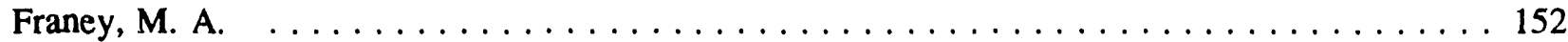

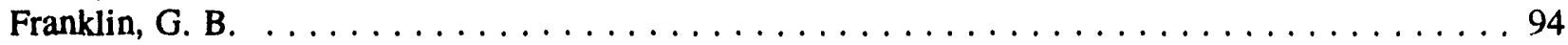

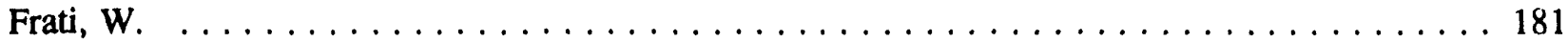

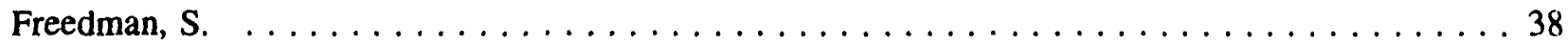

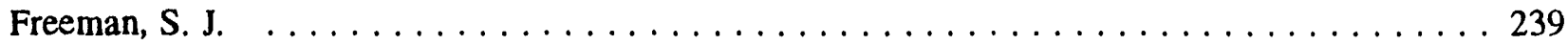

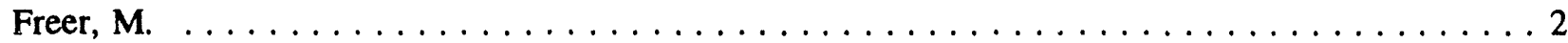

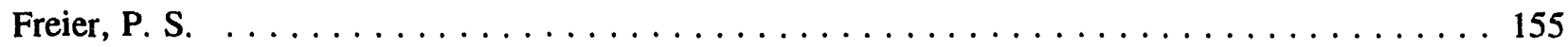

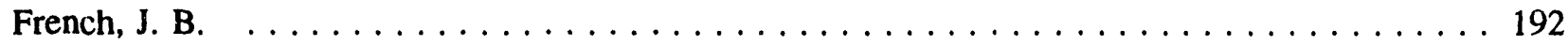

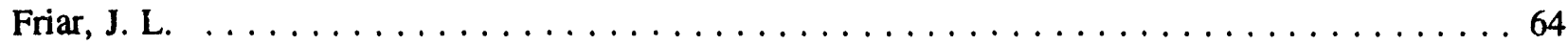

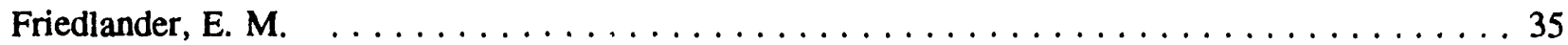

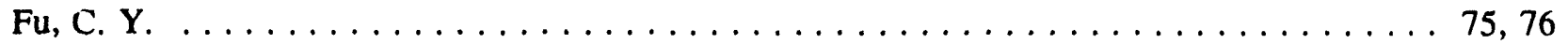

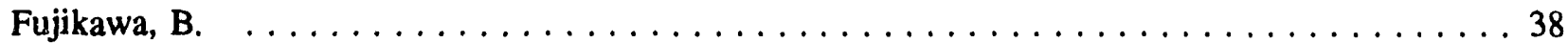

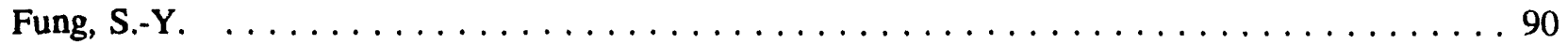

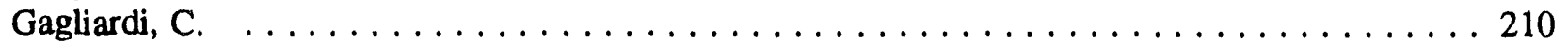

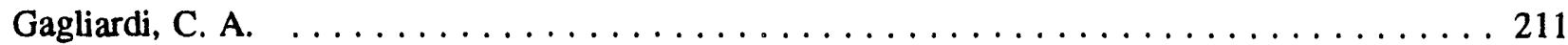

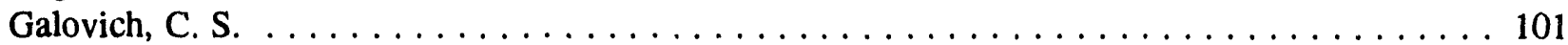

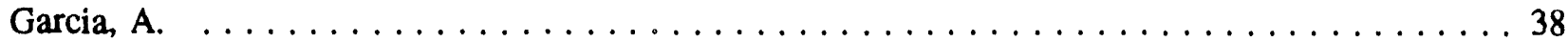

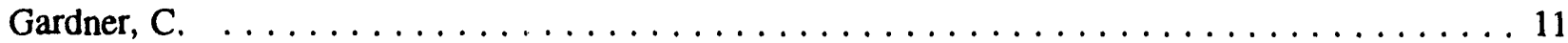

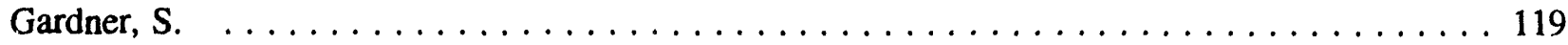

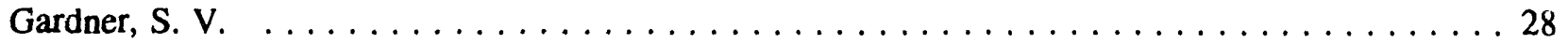

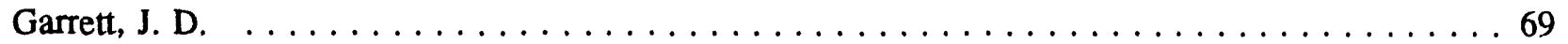

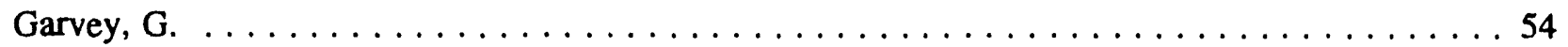

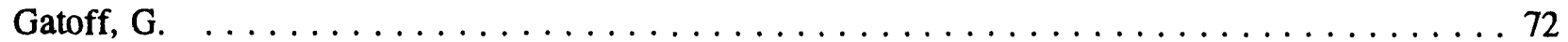

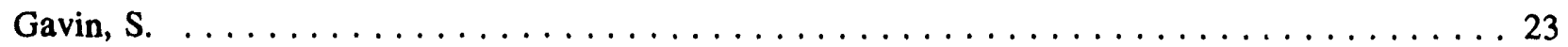

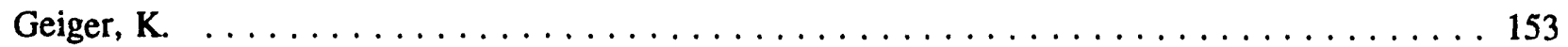

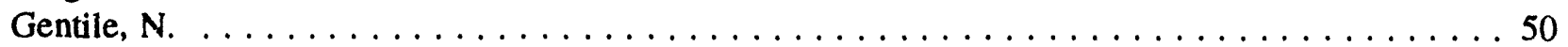

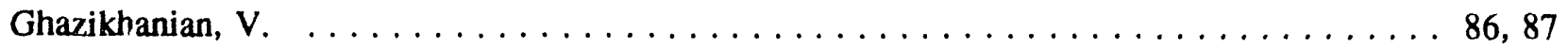

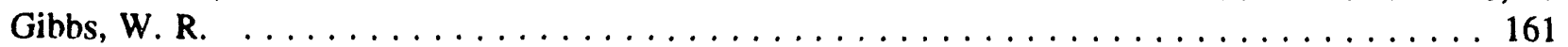

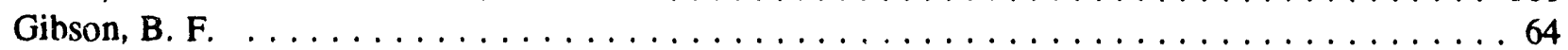

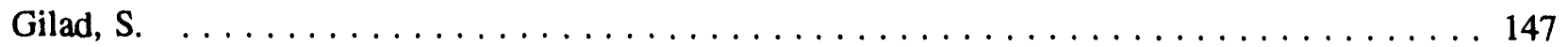

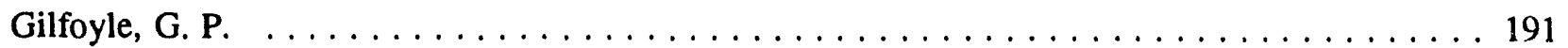

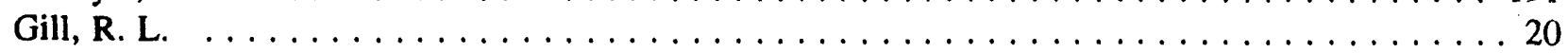

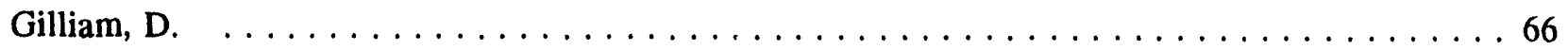

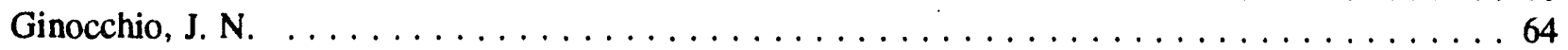

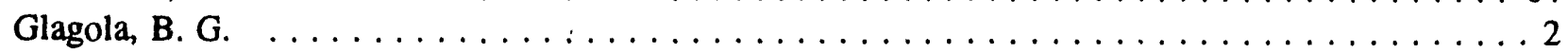

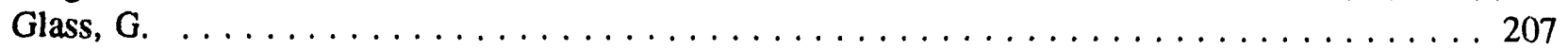

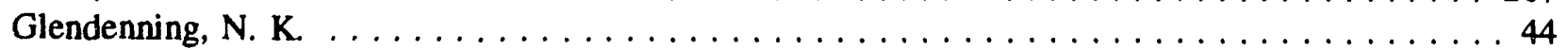

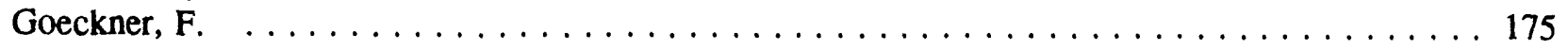

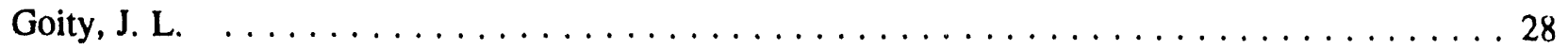

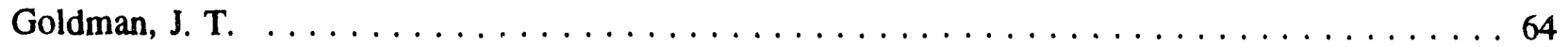

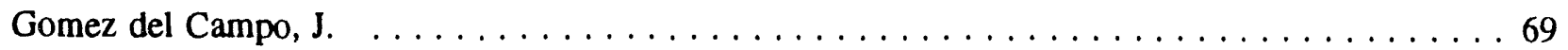

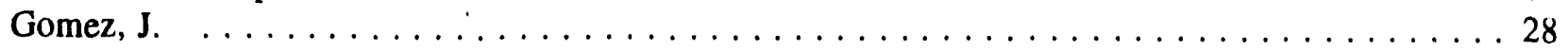

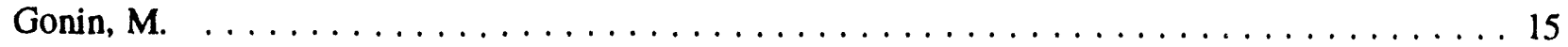




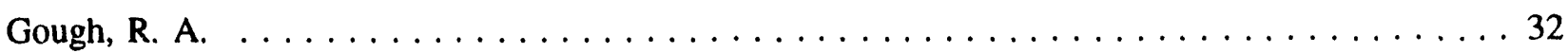

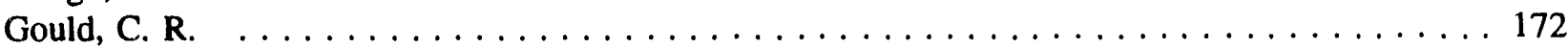

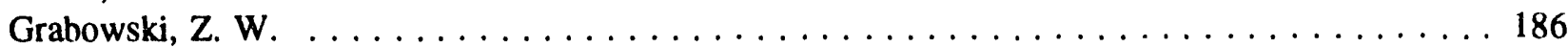

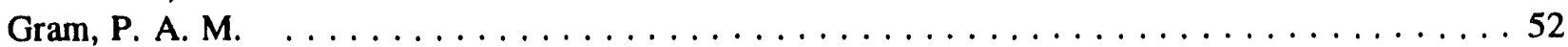

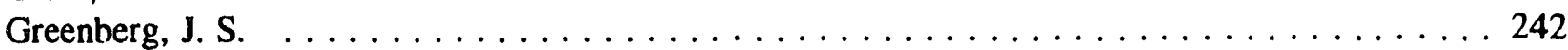

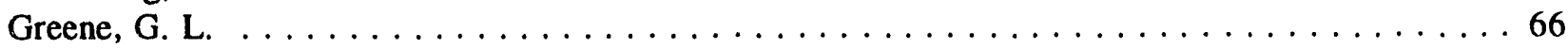

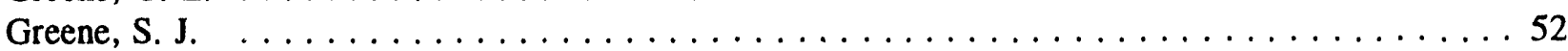

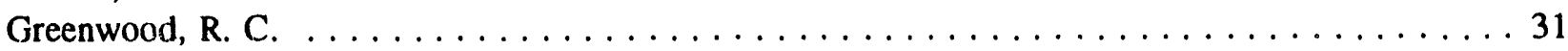

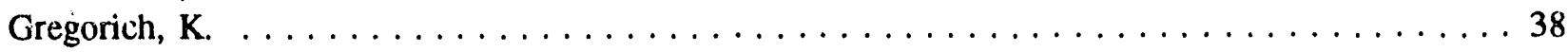

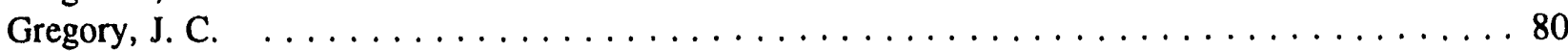

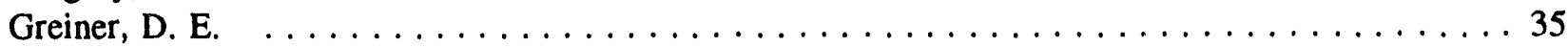

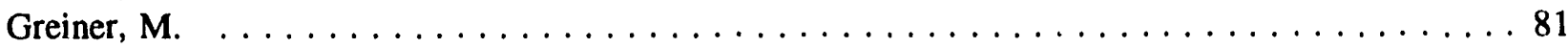

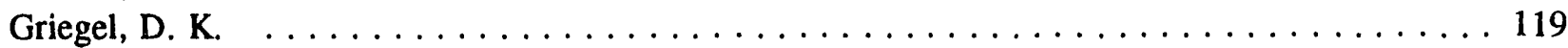

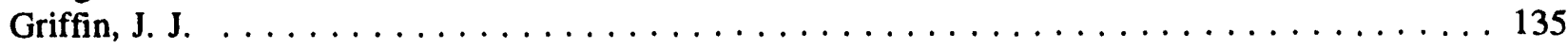

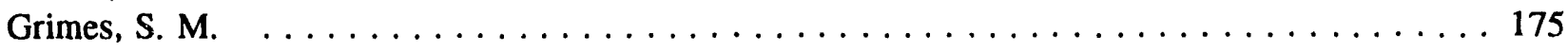

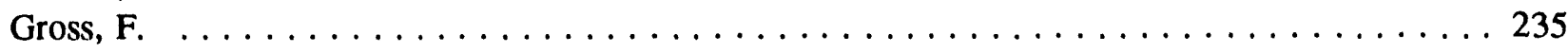

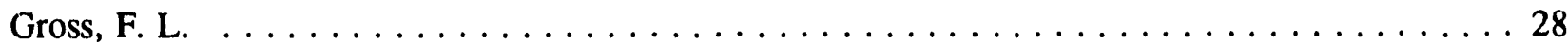

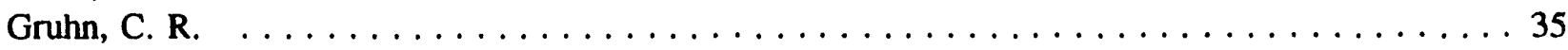

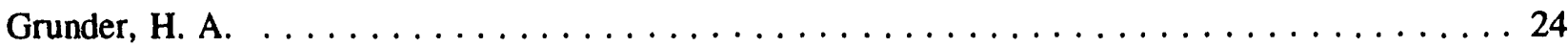

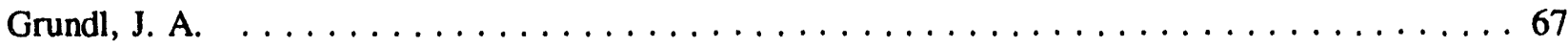

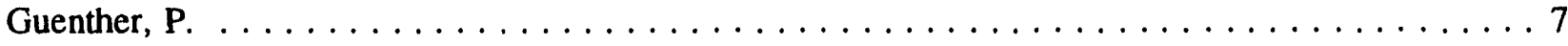

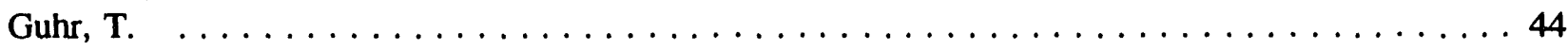

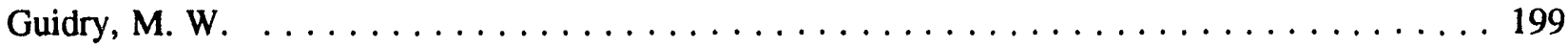

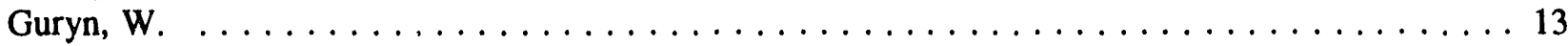

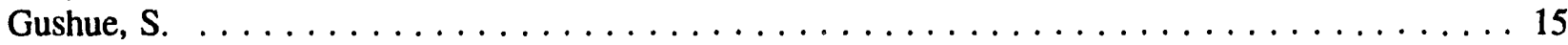

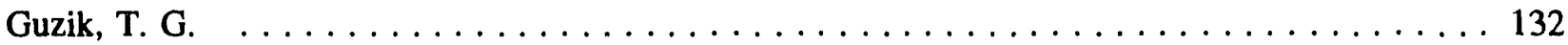

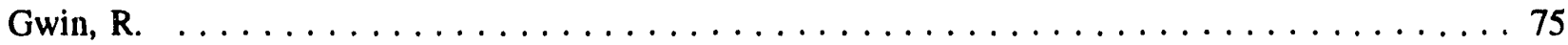

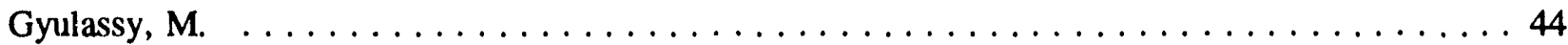

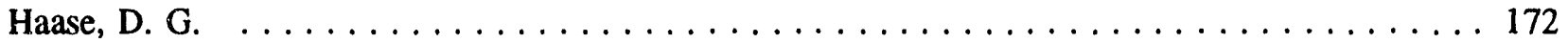

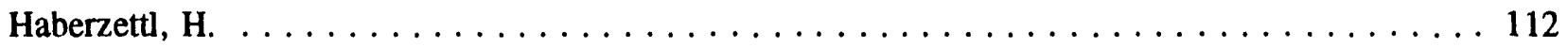

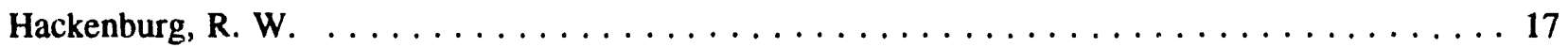

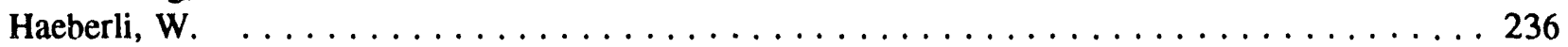

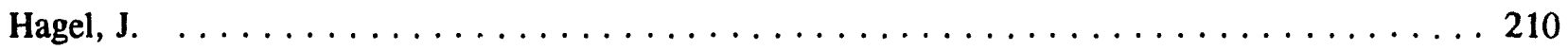

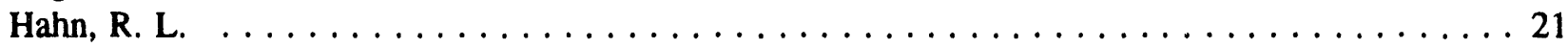

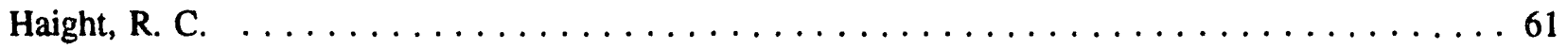

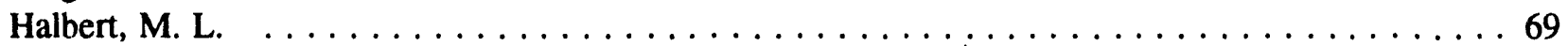

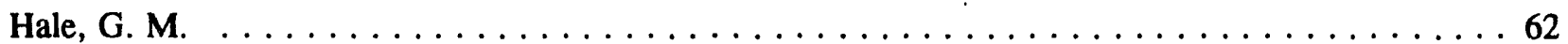

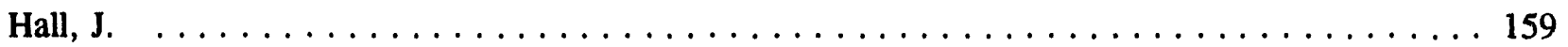

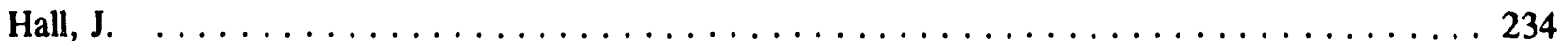

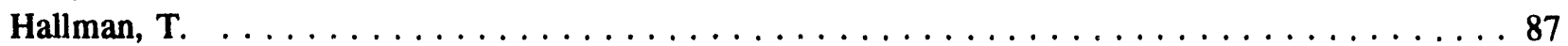

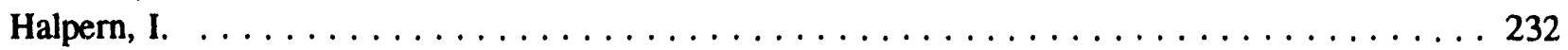

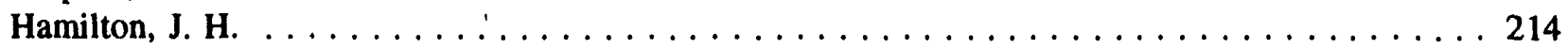

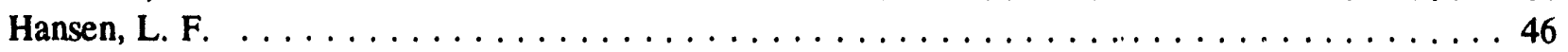

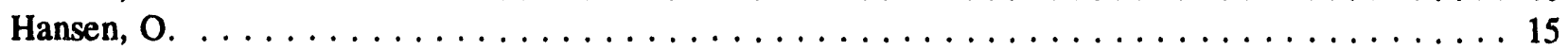

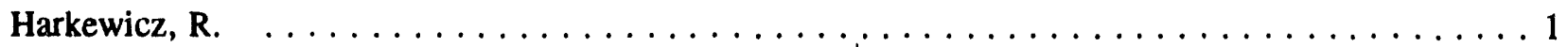

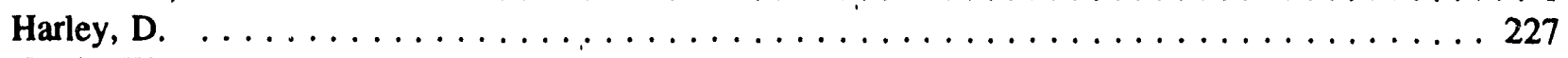

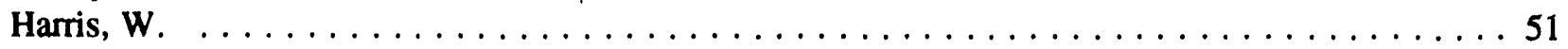

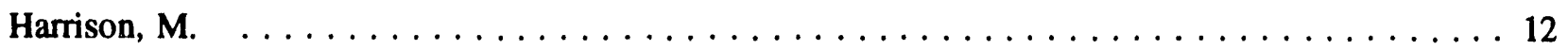

Hartline, B. ................................ 24 


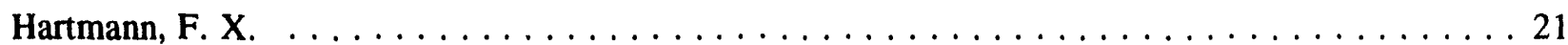

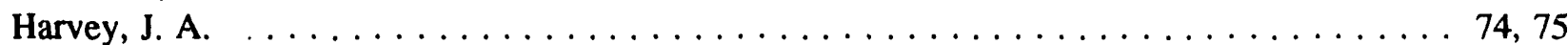

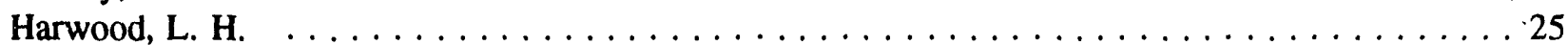

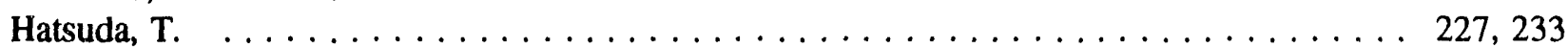

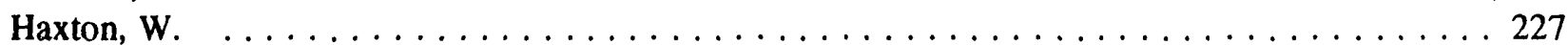

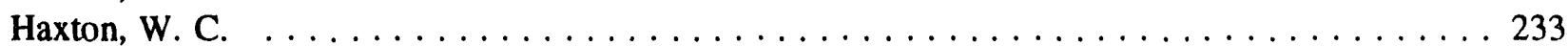

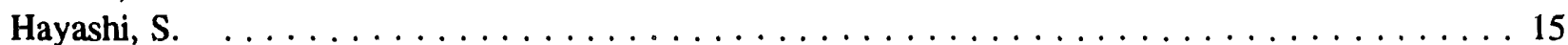

He, X. C. . . . . . . . . . . . . . . . . . . . . . . . . . . . . . . 199

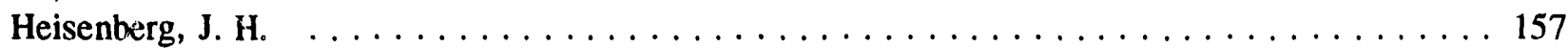

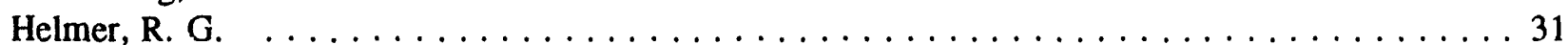

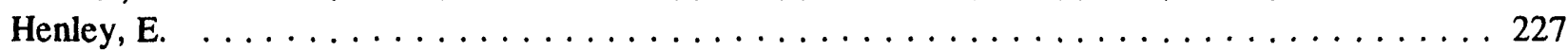

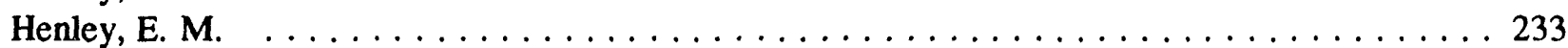

Henning, W. F. $\ldots \ldots \ldots \ldots \ldots \ldots \ldots \ldots \ldots \ldots \ldots \ldots \ldots \ldots \ldots \ldots \ldots \ldots, 2,4,6$

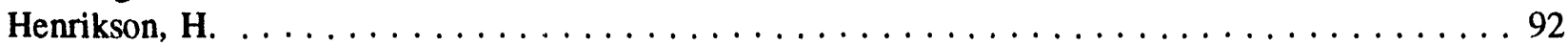

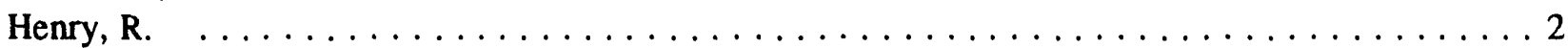

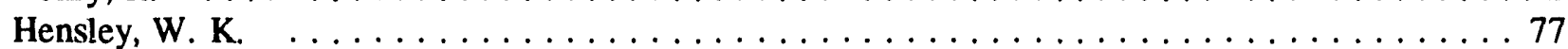

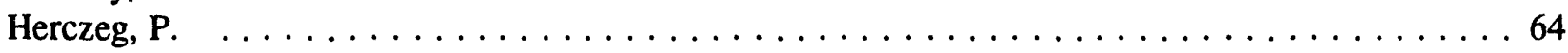

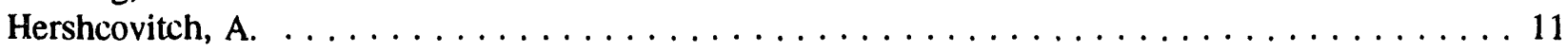

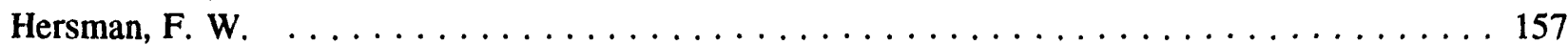

Hetrick, D. M. $\ldots \ldots \ldots \ldots \ldots \ldots \ldots \ldots \ldots \ldots \ldots \ldots \ldots \ldots \ldots \ldots \ldots \ldots \ldots$

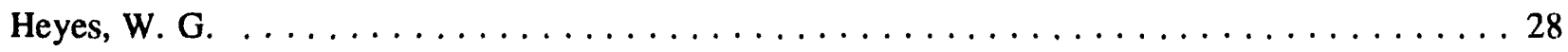

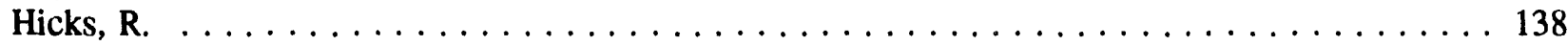

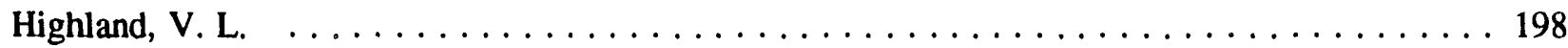

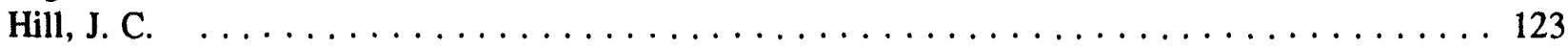

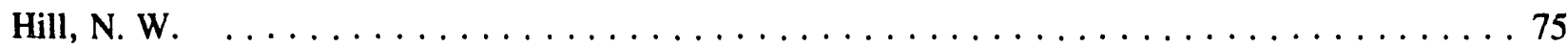

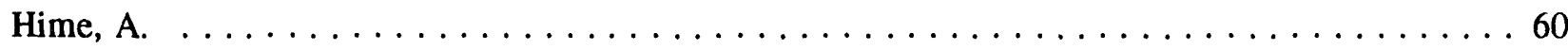

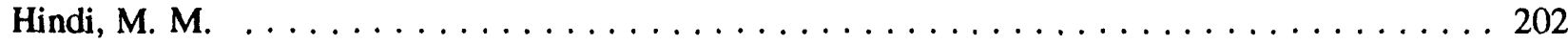

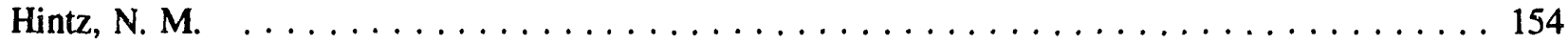

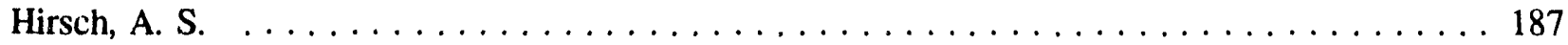

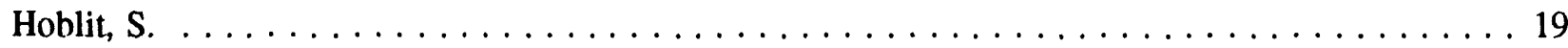

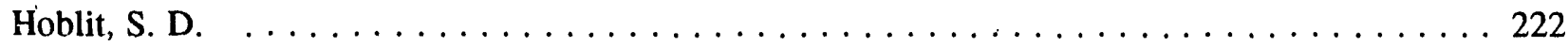

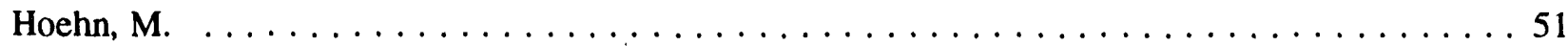

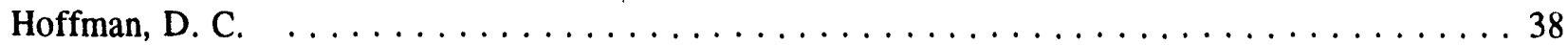

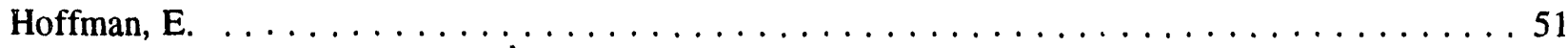

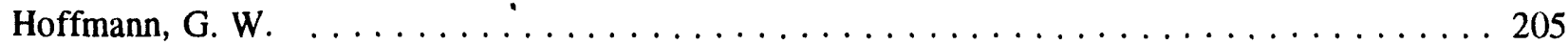

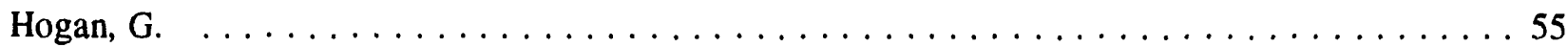

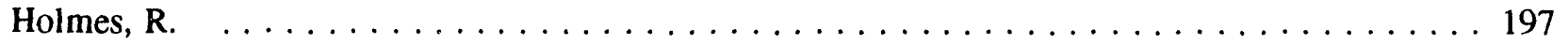

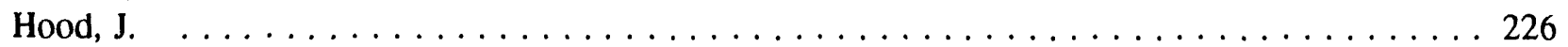

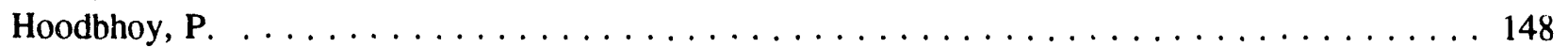

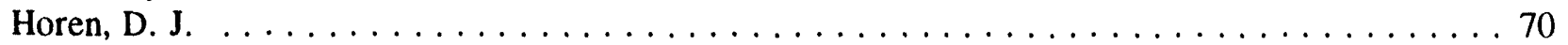

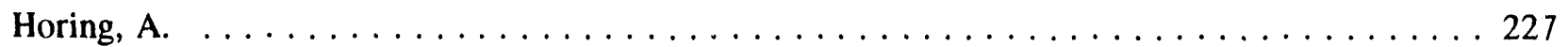

Horowitz, C. J. . . . . . . . . . . . . . . . . . . . . . . . . . . . . . . . . 119

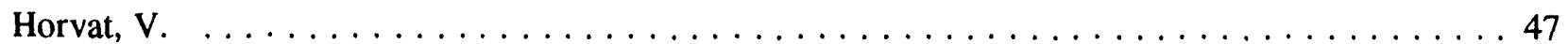

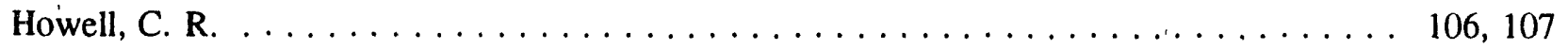

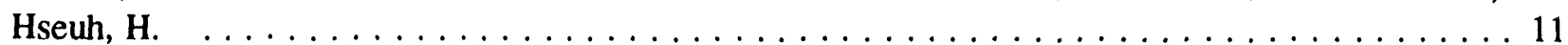

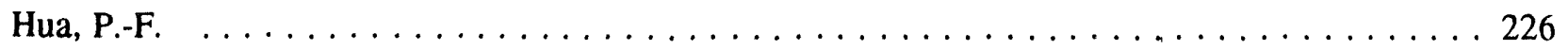

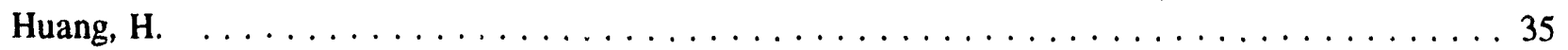

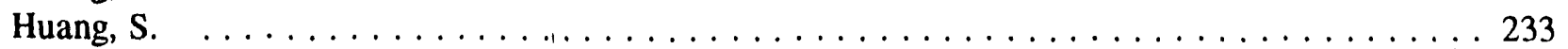

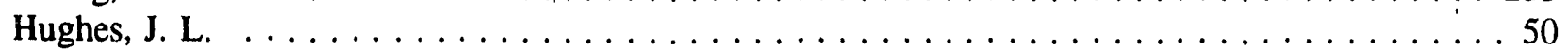

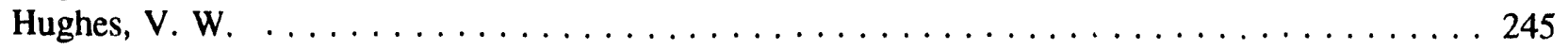


Huizenga, J. R. . . . . . . . . . . . . . . . . . . . . . . . . . . . . . 193

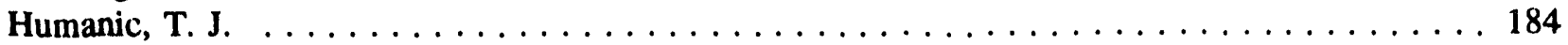

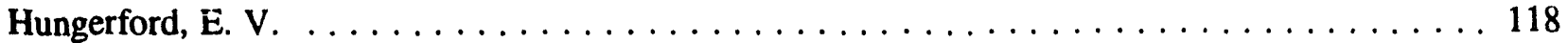

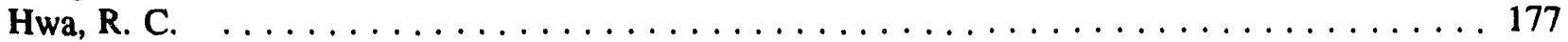

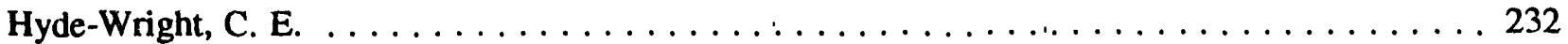

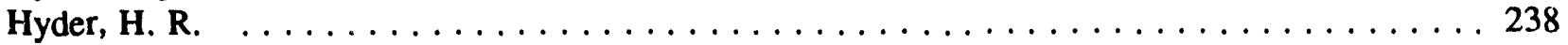

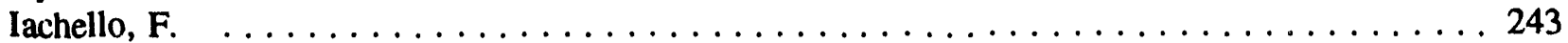

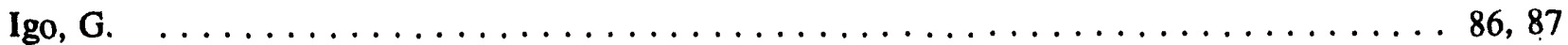

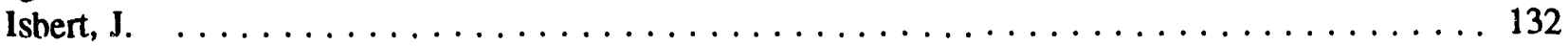

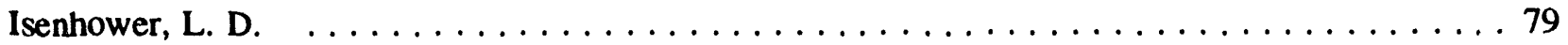

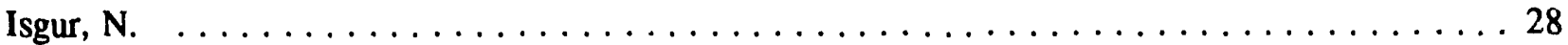

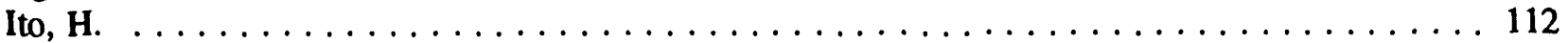

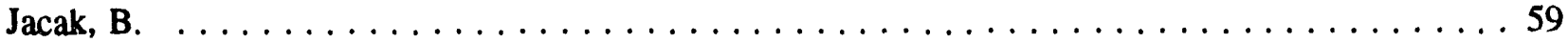

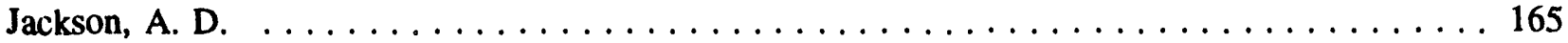

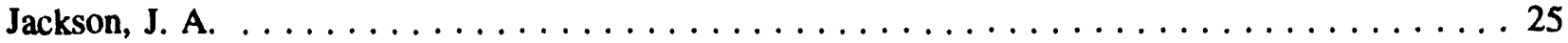

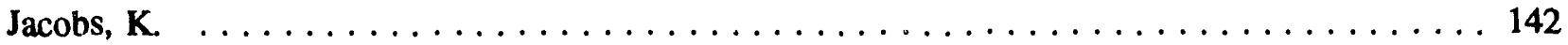

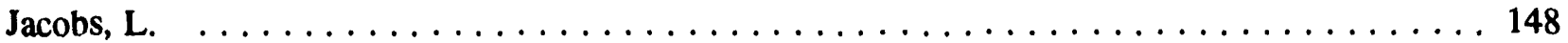

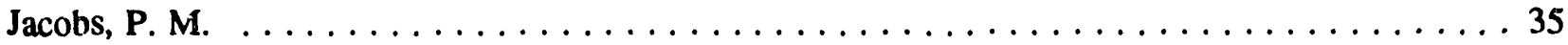

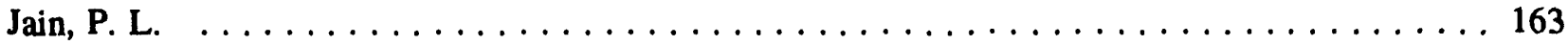

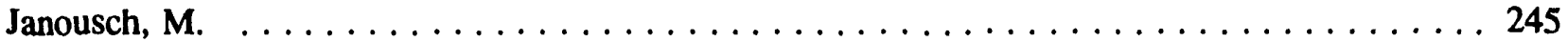

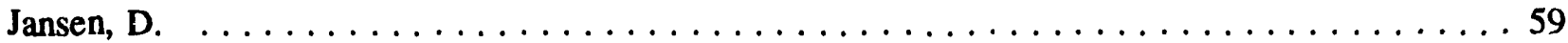

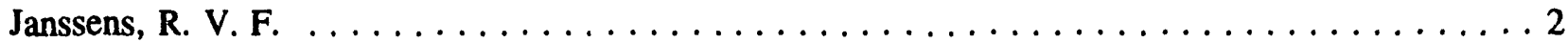

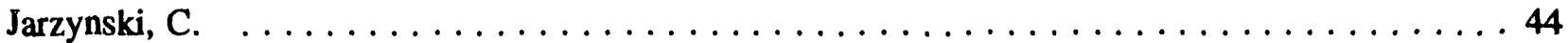

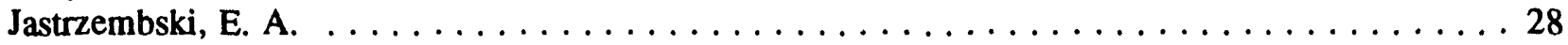

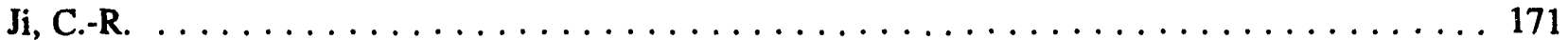

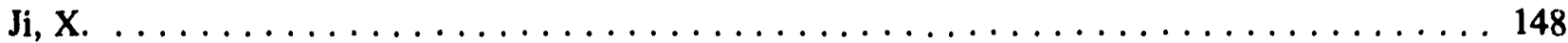

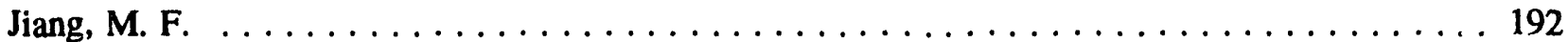

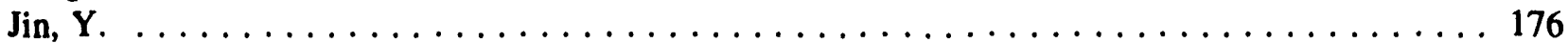

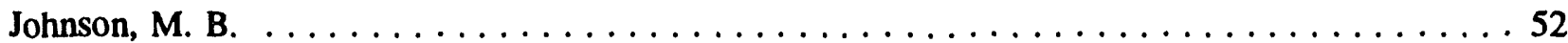

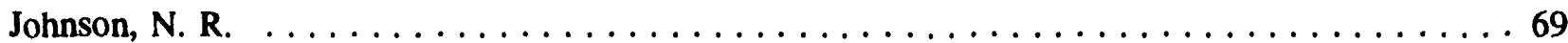

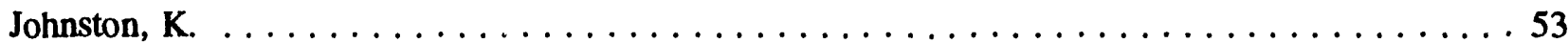

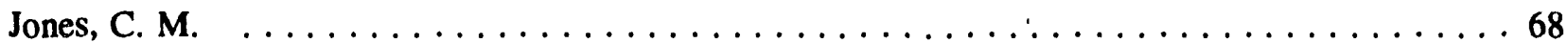

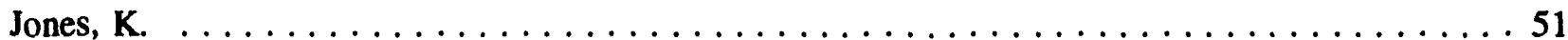

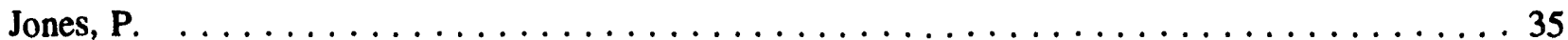

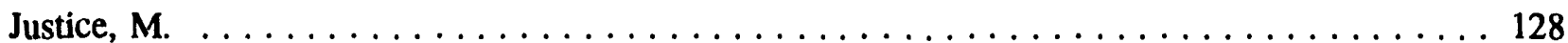

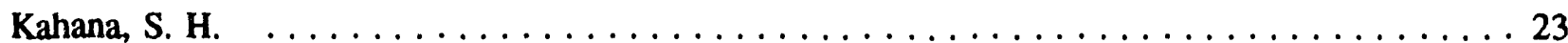

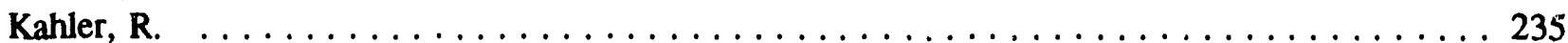

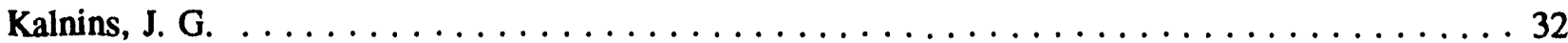

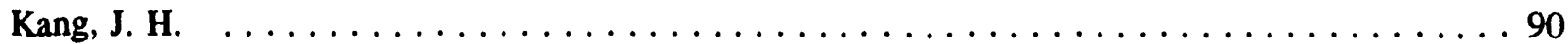

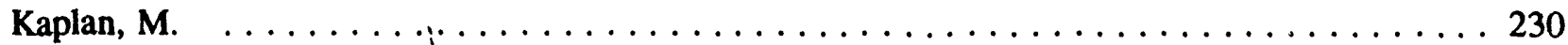

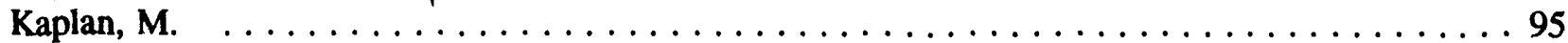

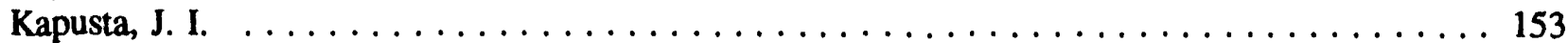

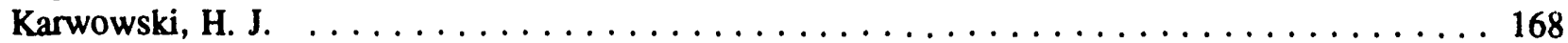

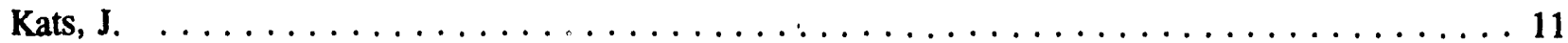

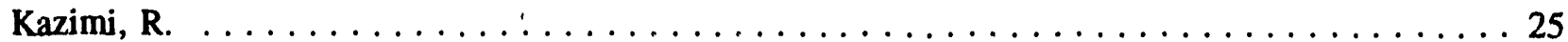

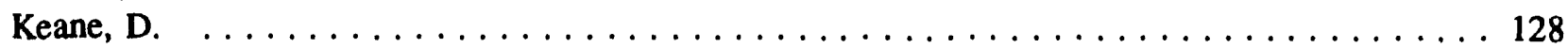

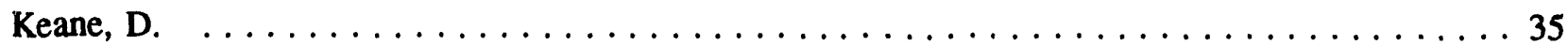

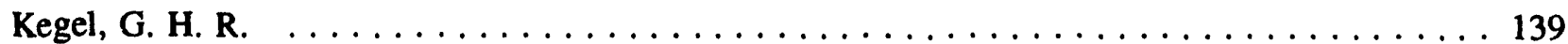

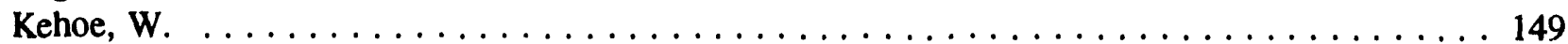




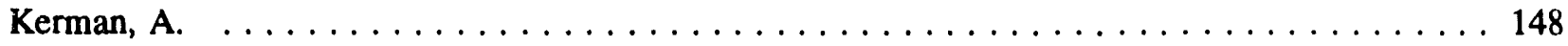

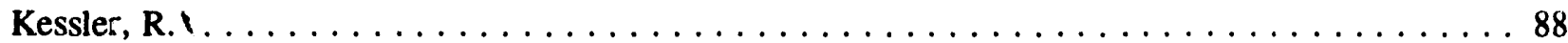

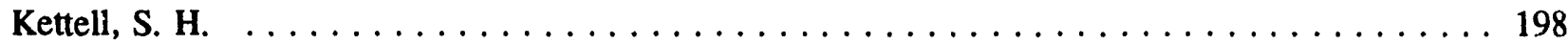

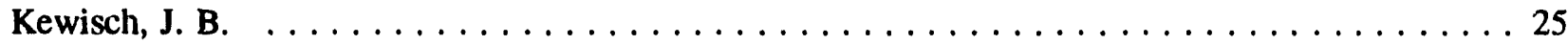

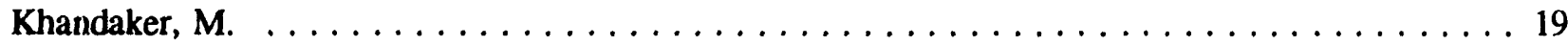

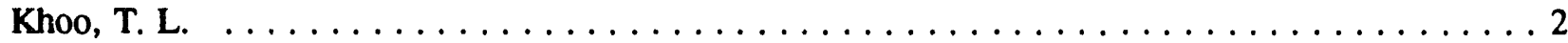

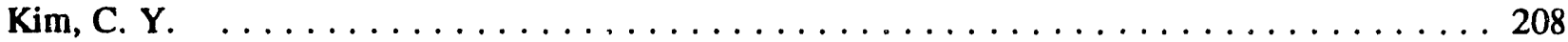

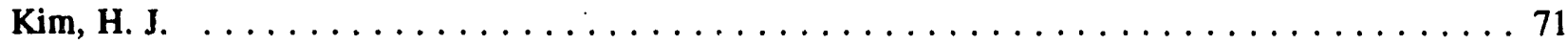

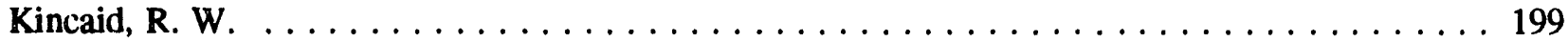

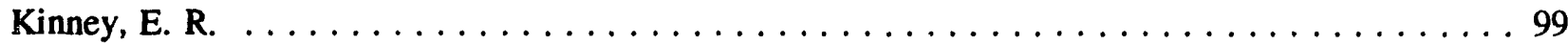

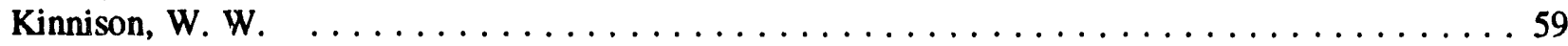

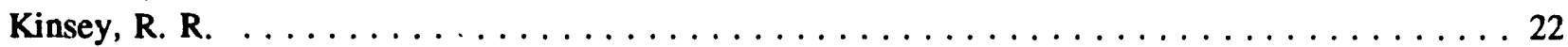

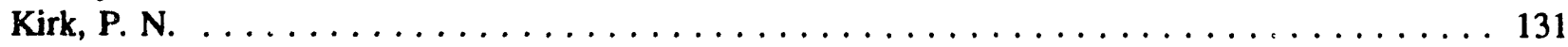

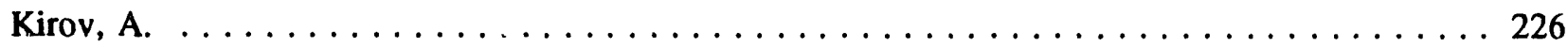

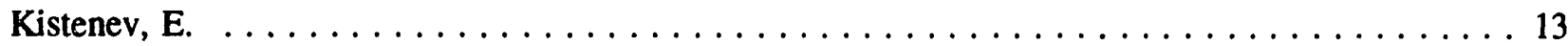

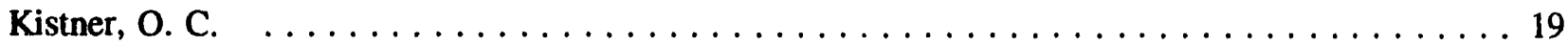

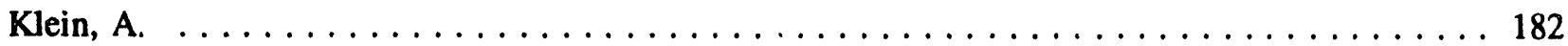

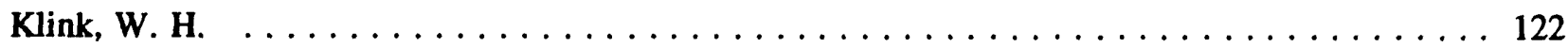

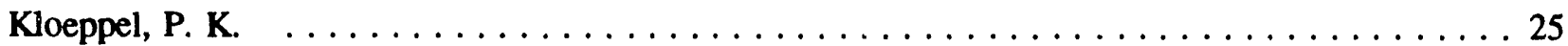

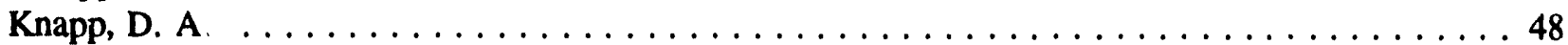

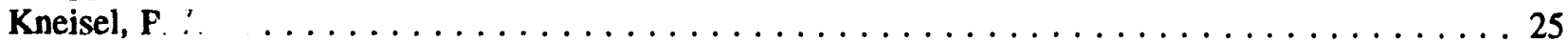

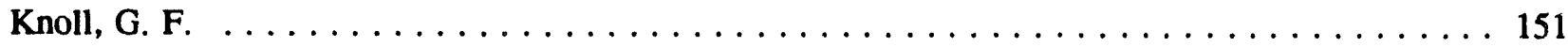

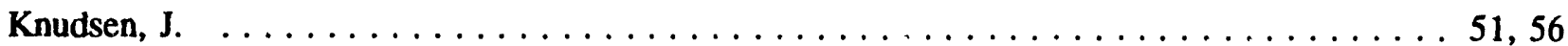

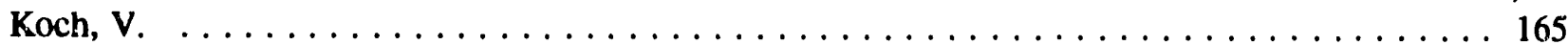

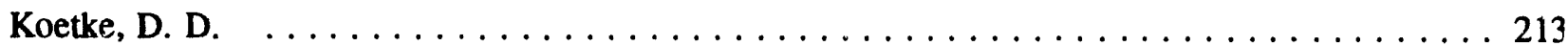

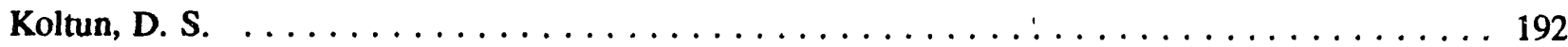

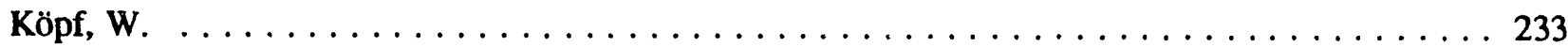

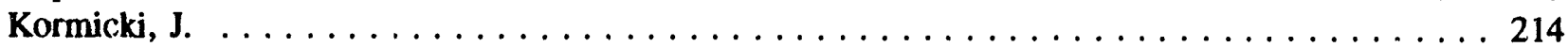

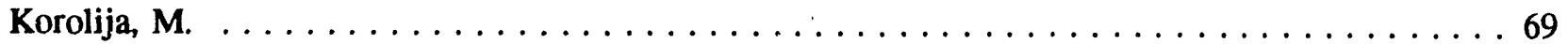

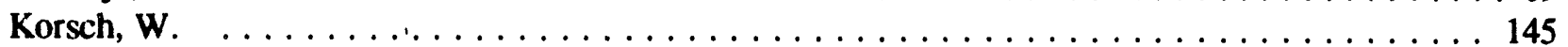

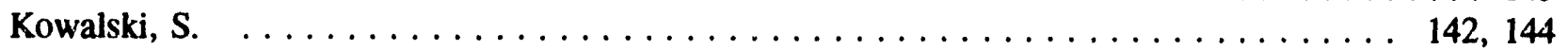

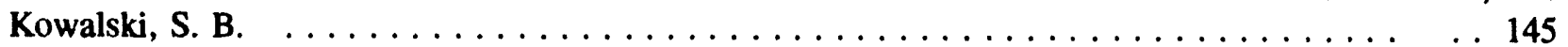

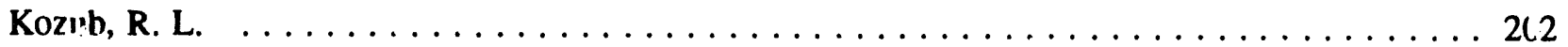

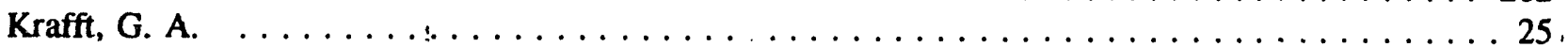

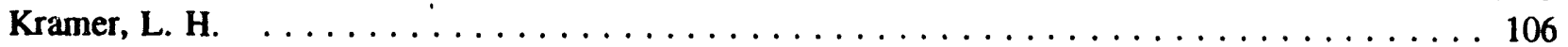

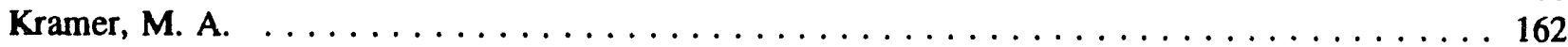

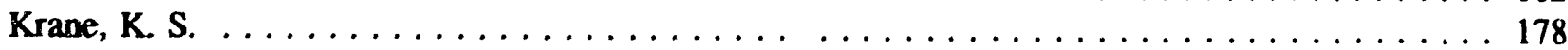

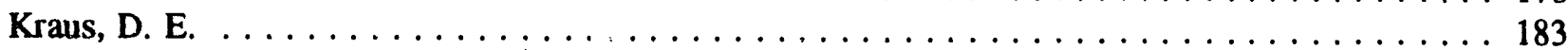

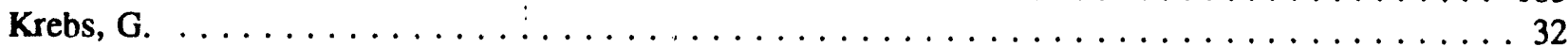

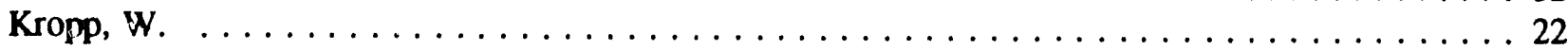

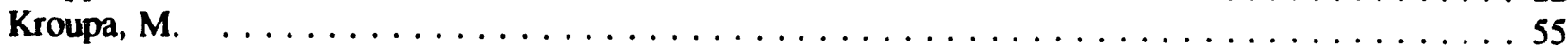

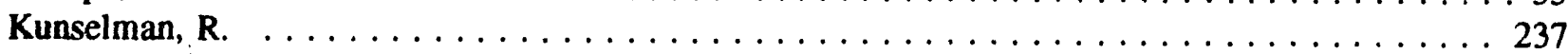

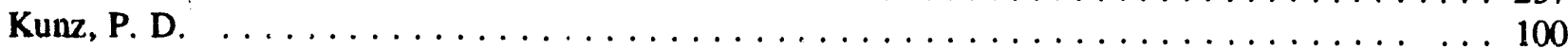

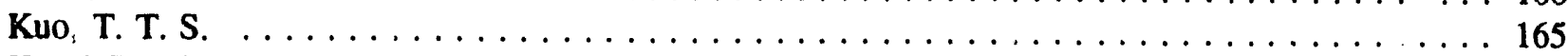

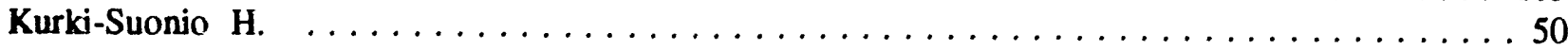

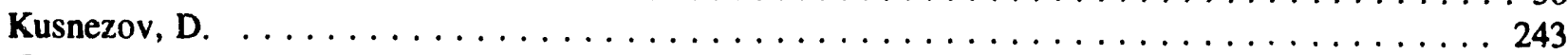

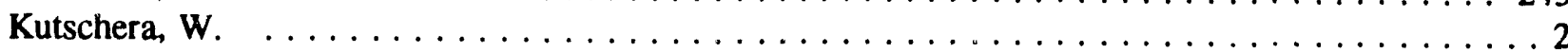

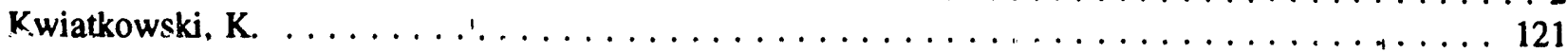

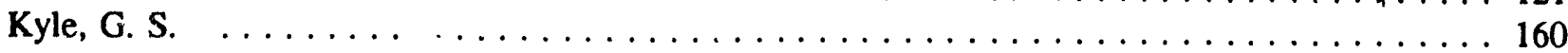

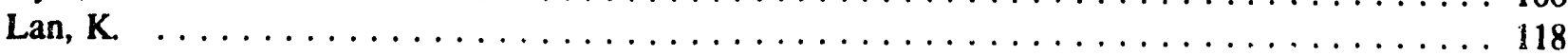


Landau, R. H. . . . . . . . . . . . . . . . . . . . . . . 179

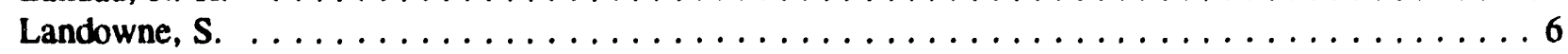

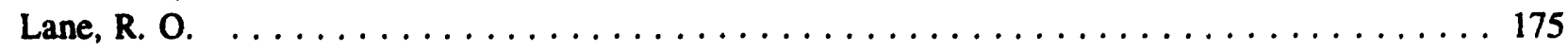

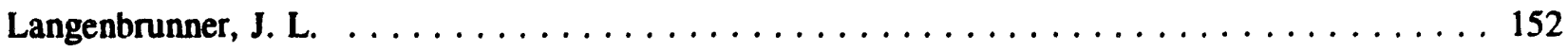

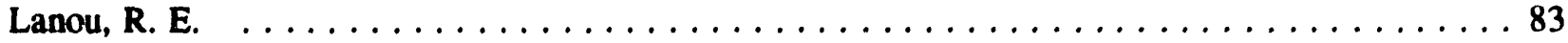

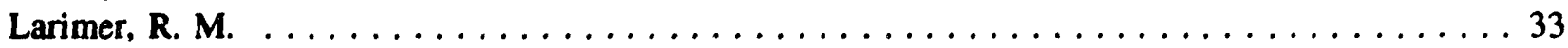

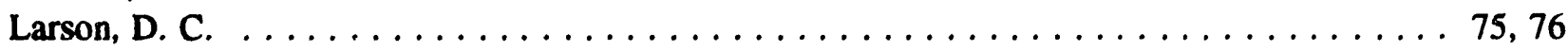

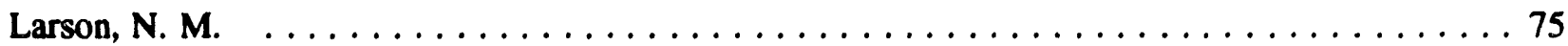

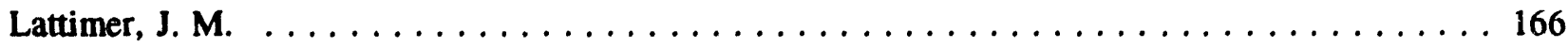

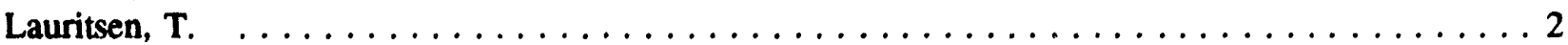

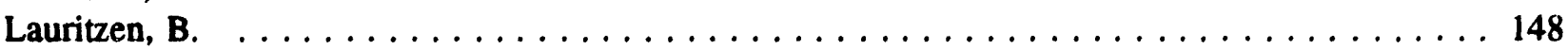

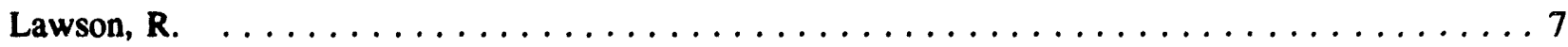

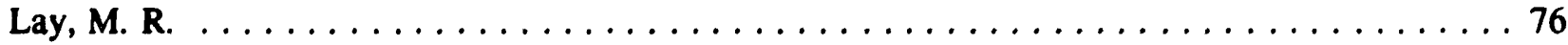

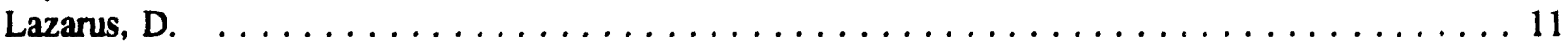

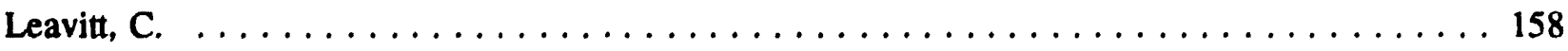

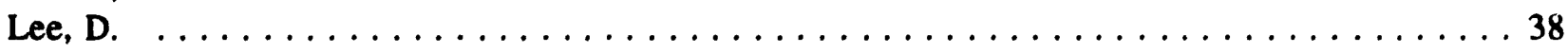

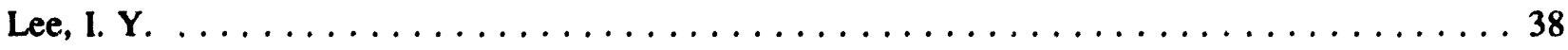

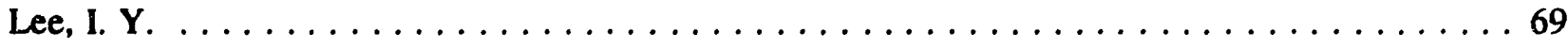

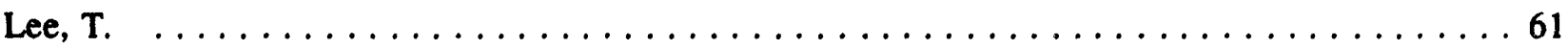

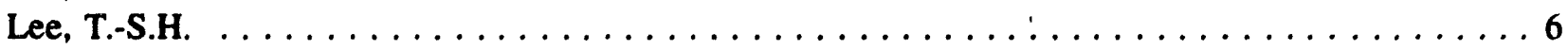

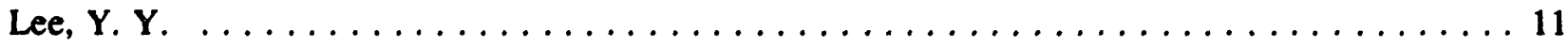

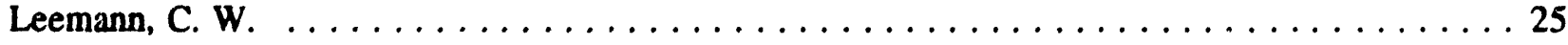

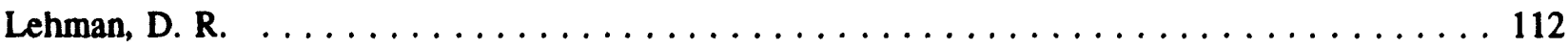

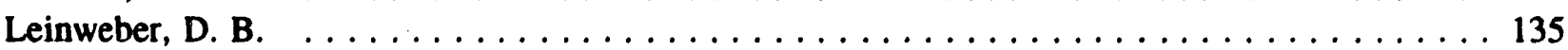

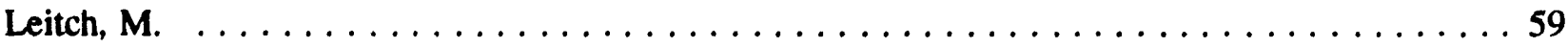

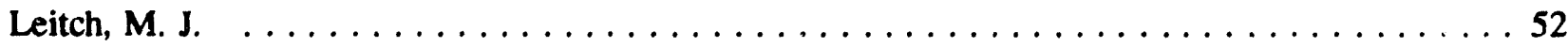

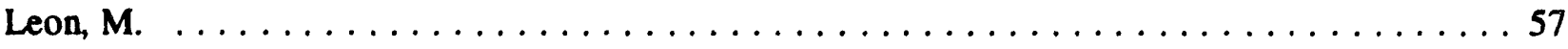

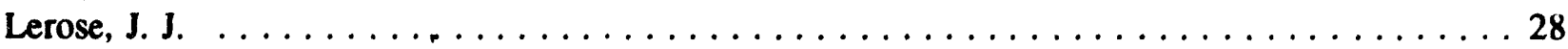

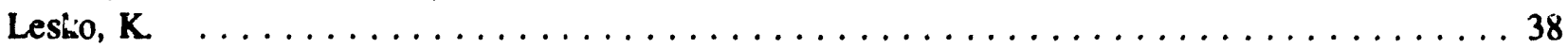

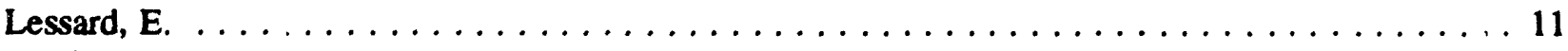

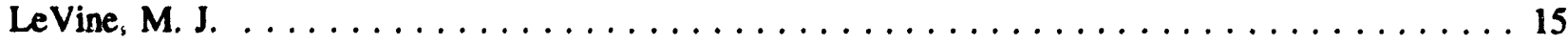

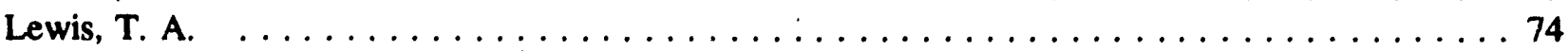

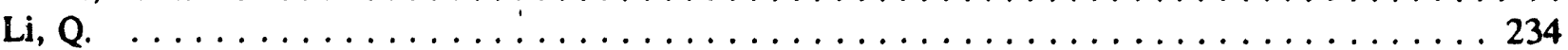

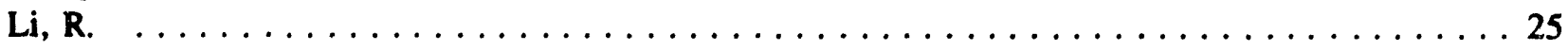

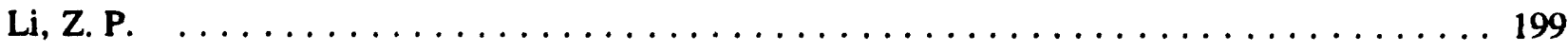

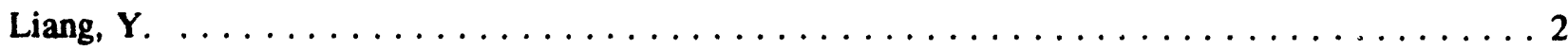

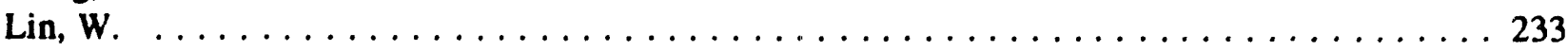

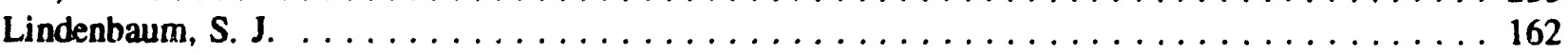

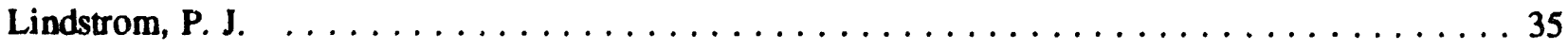

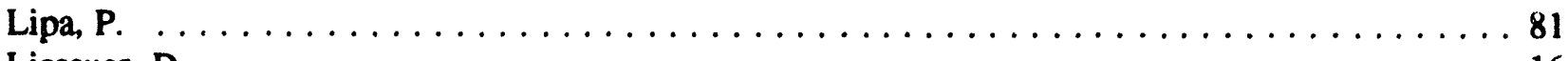

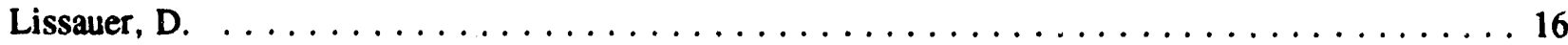

Lister, C. J. . . . . . . . . . . . . . . . . . . . . . . . . . . . . 239, 242

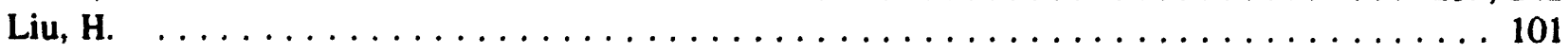

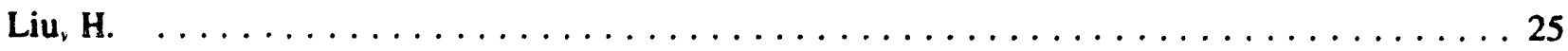

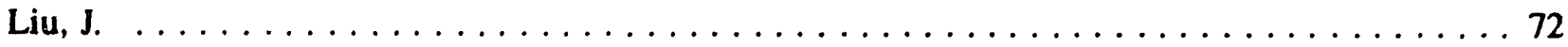

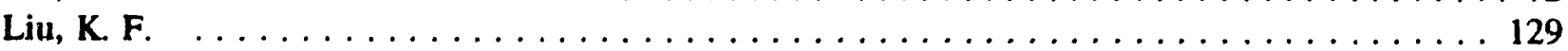

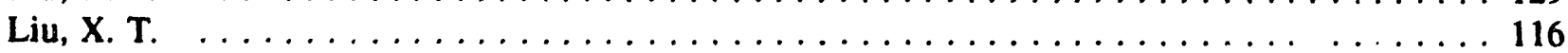

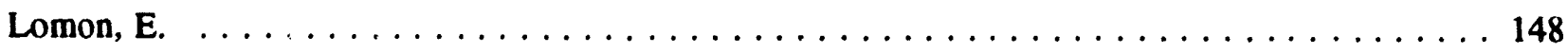

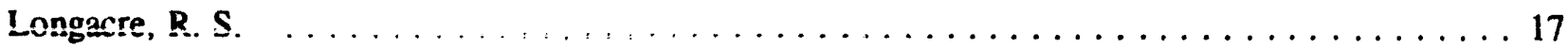


Lothrop, F.

Lott, $B$.

Louis, W.

Lourie, R. W.

Love, W. A.

Loveland, W.

Lowenstein, D.

Lu, H. J.

Luccio, A.

Ludewigt, B.

Ludlam, T.

Ludlam, T. W.

Ludwig, E. J.

Lui, Y.-W.

Luo, $M$.

Luo, $\mathbf{M}$.

Lutz, $M$.

Lyneis, C. M

Ma, W. C.

Macchevelli, A.

MacDonald, W. M.

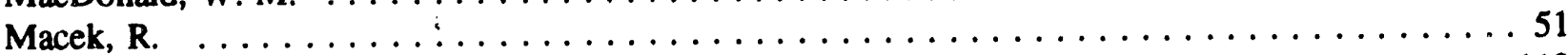

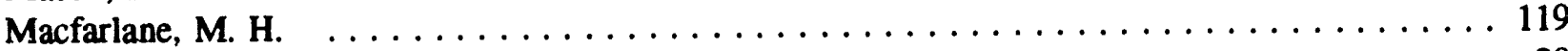

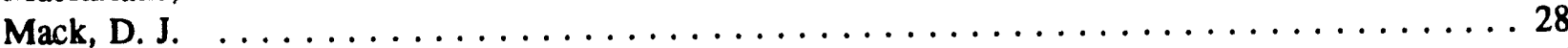

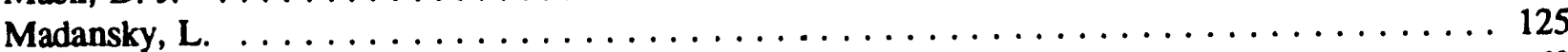

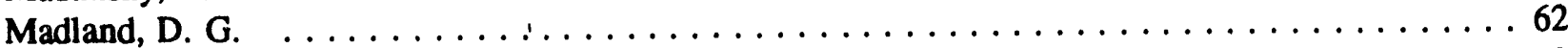

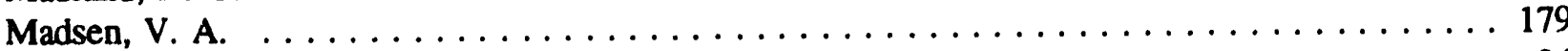

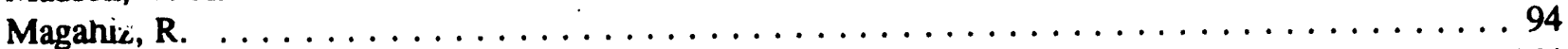

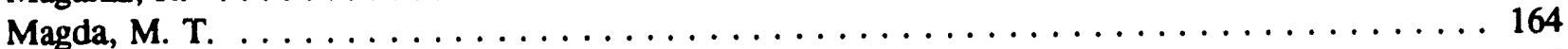

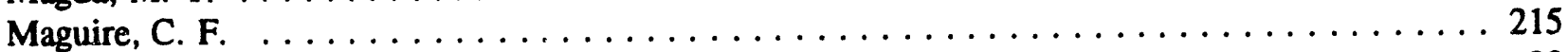

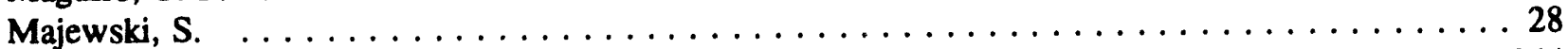

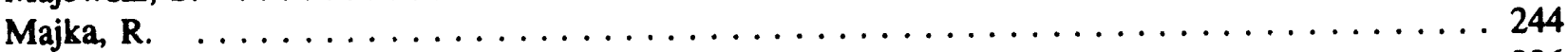

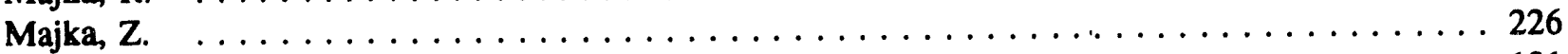

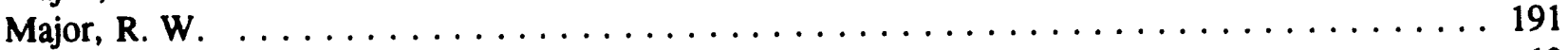

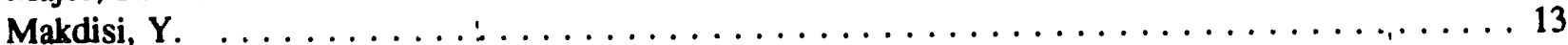

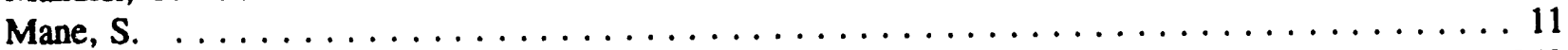

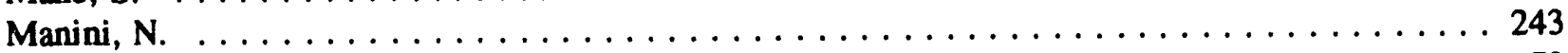

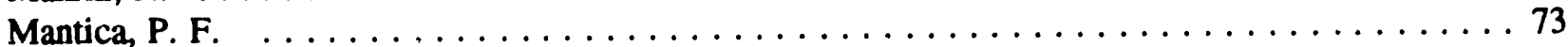

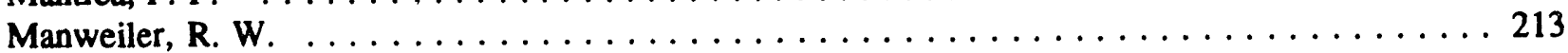

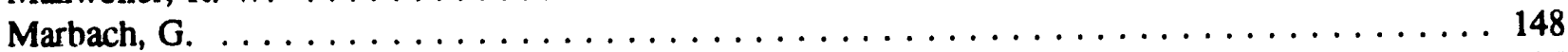

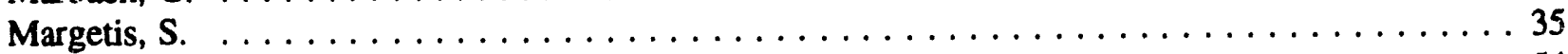

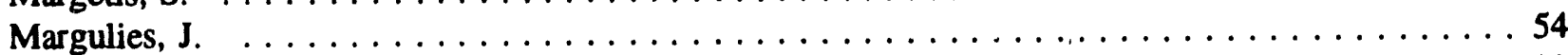

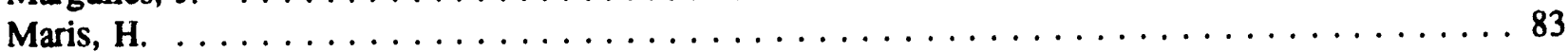

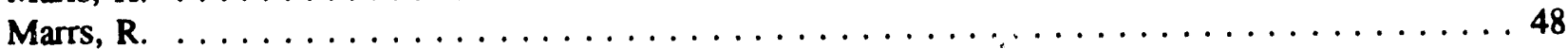

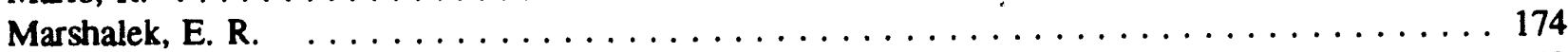

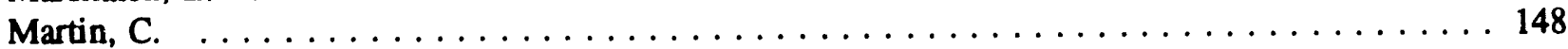

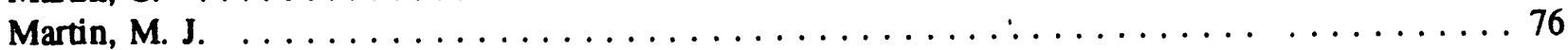

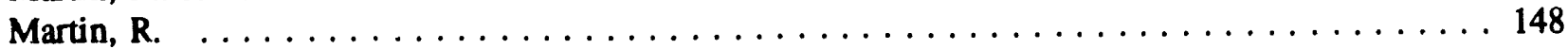

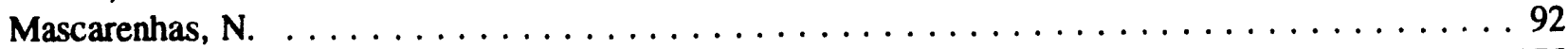

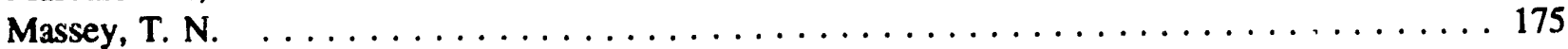




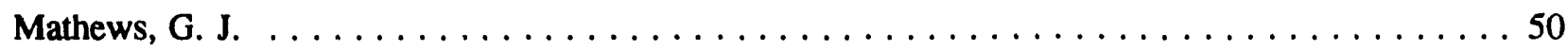

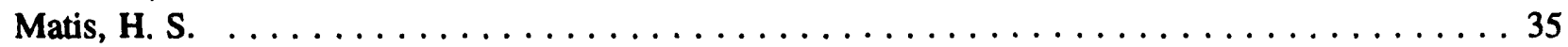

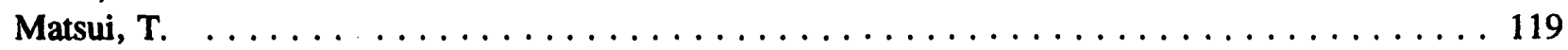

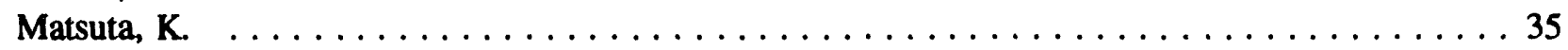

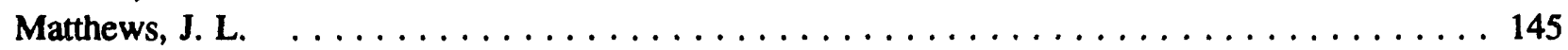

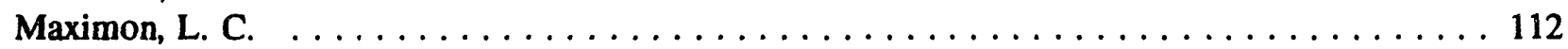

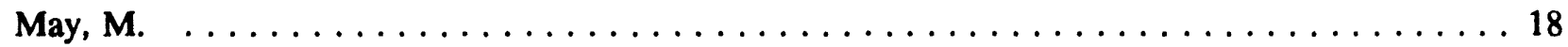

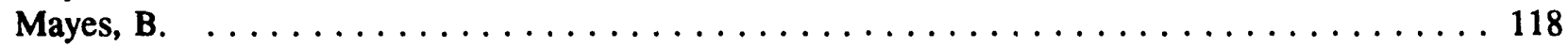

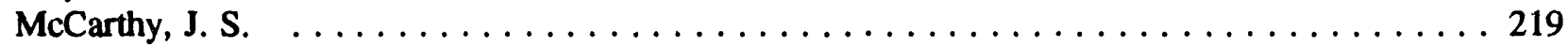

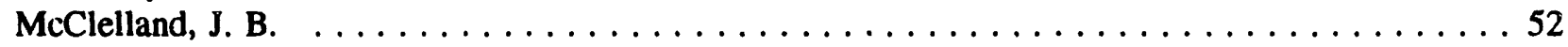

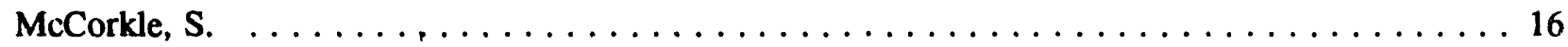

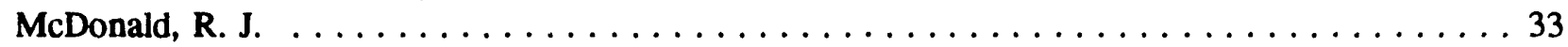

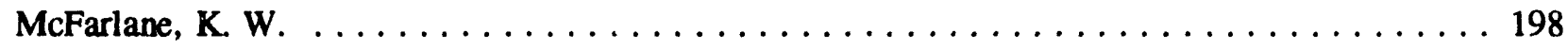

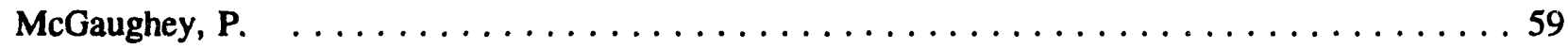

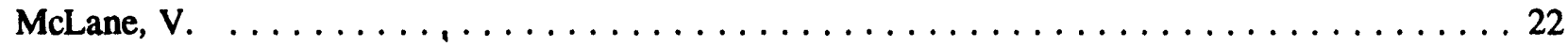

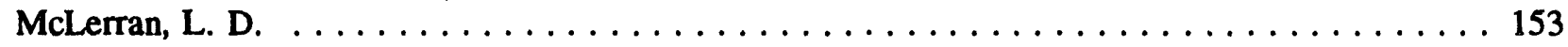

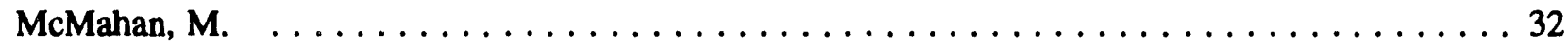

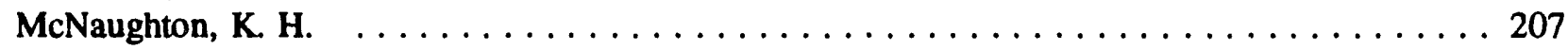

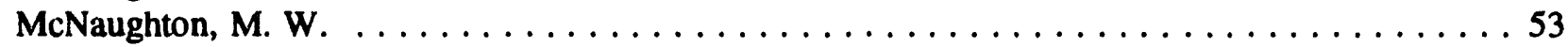

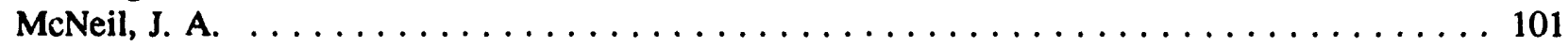

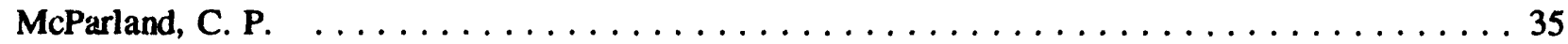

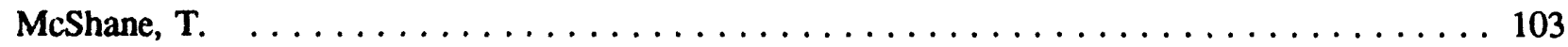

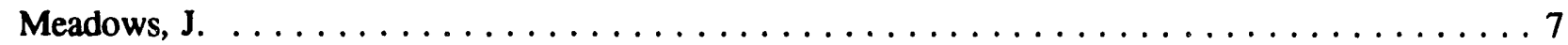

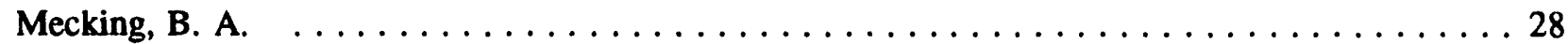

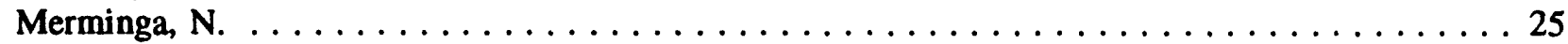

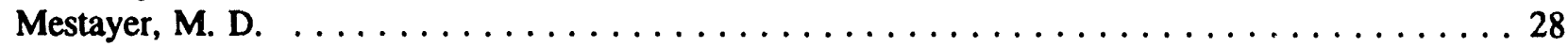

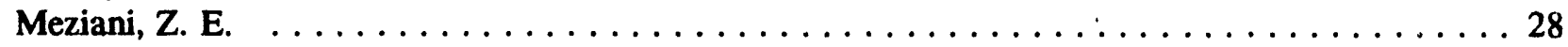

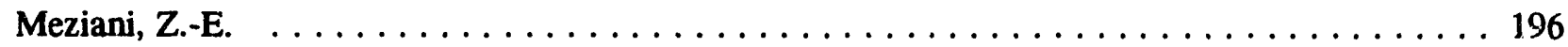

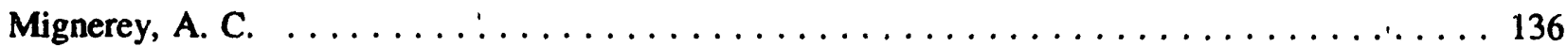

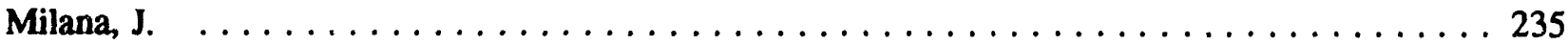

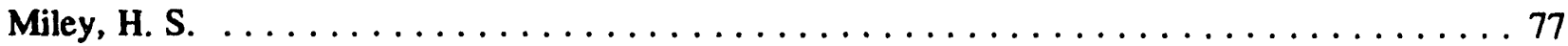

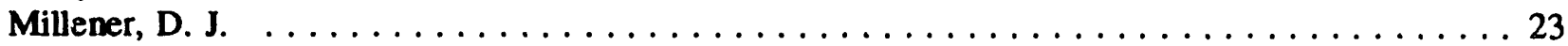

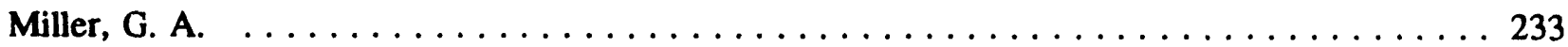

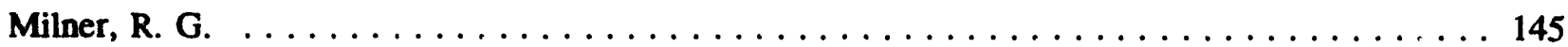

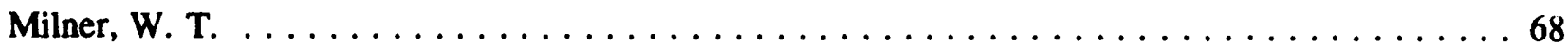

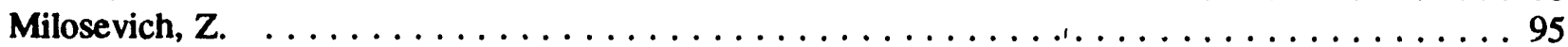

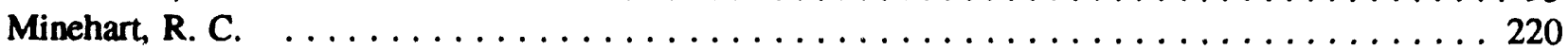

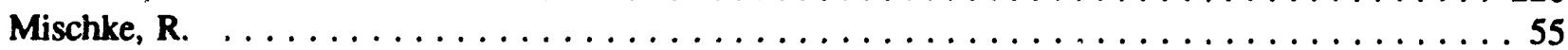

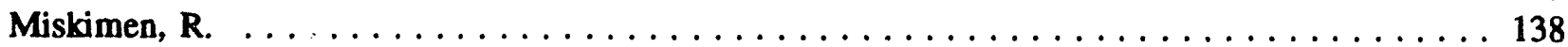

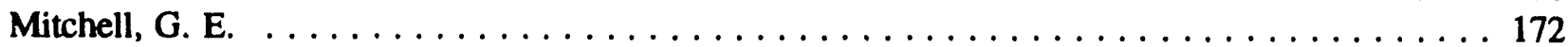

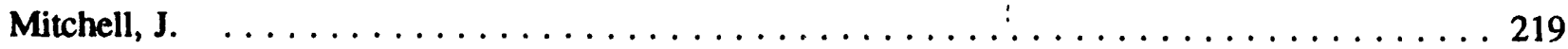

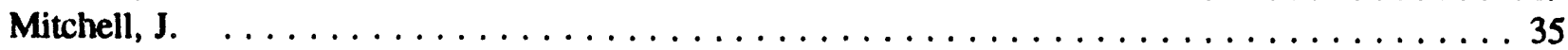

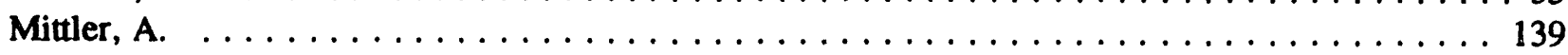

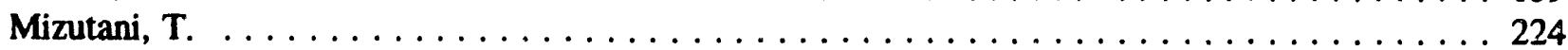

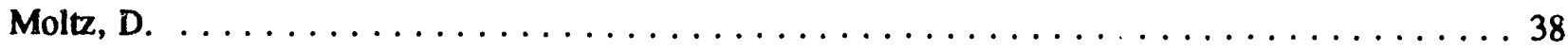

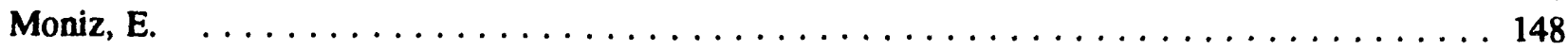

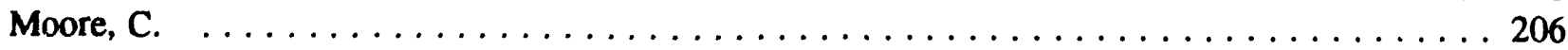

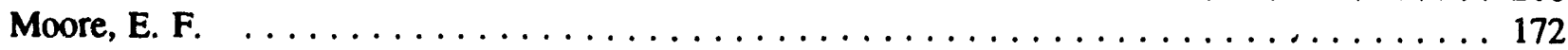

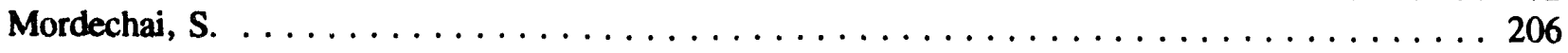




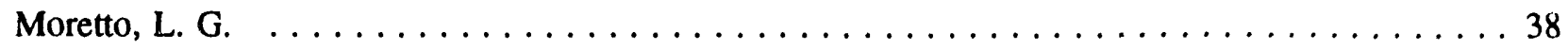

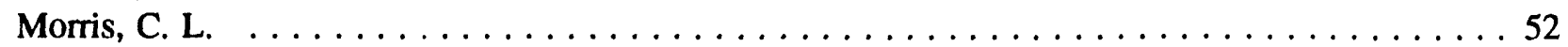

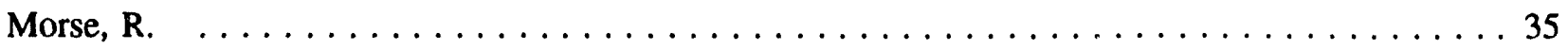

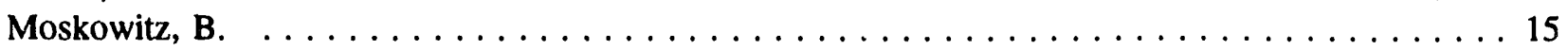

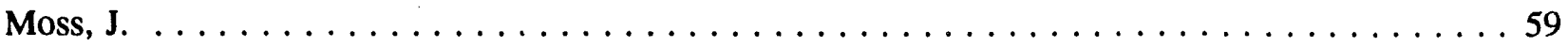

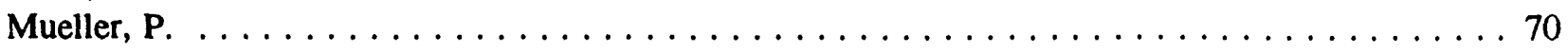

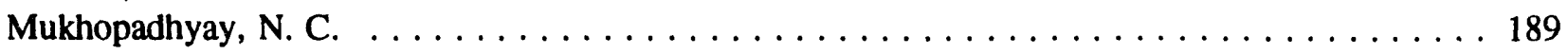

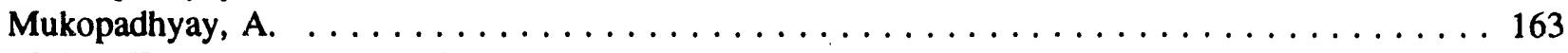

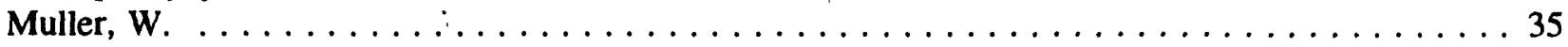

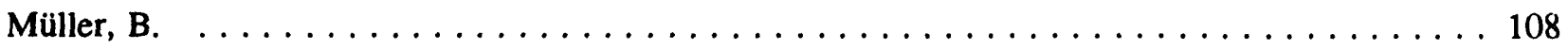

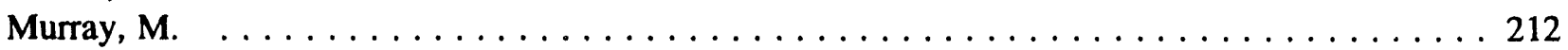

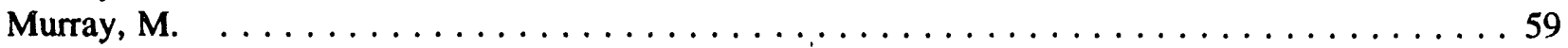

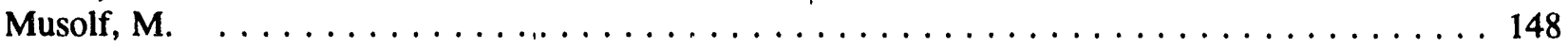

Mutchler, G. S. . . . . . . . . . . . . . . . . . . . . . . . . . . . . . . . . 190

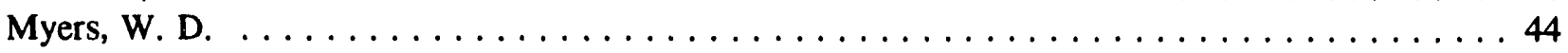

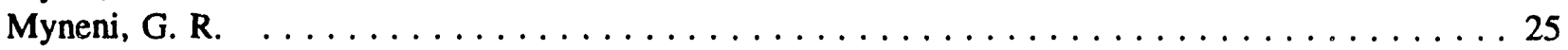

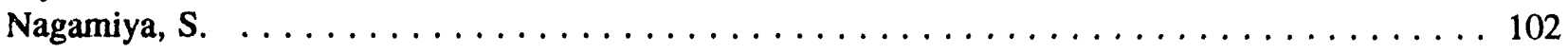

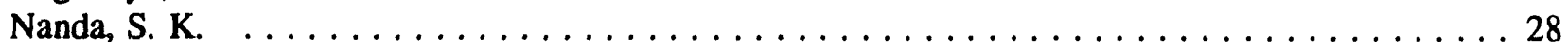

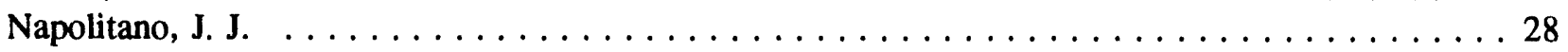

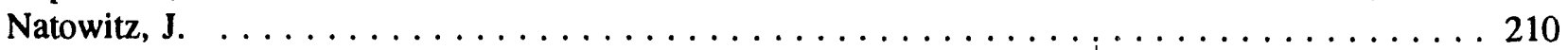

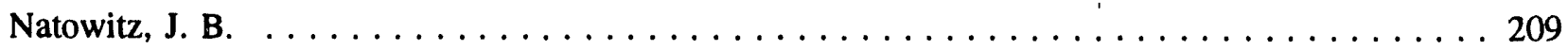

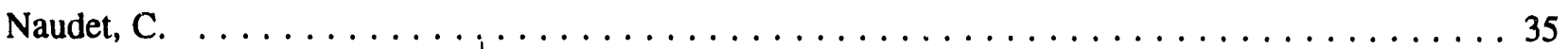

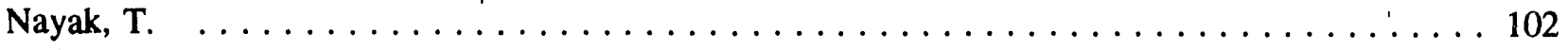

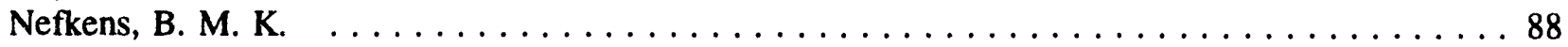

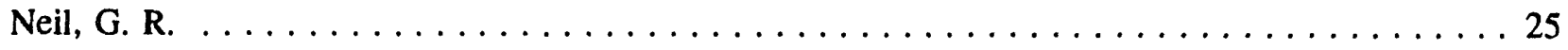

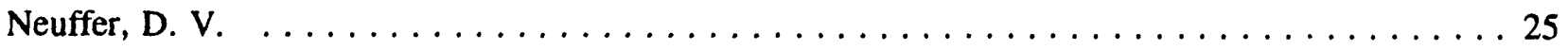

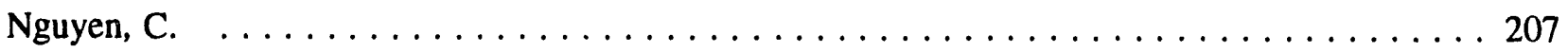

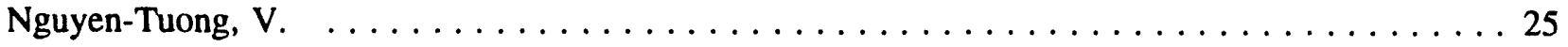

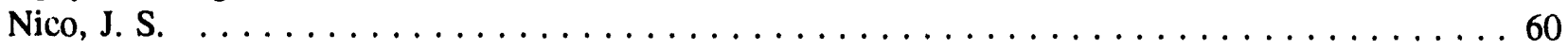

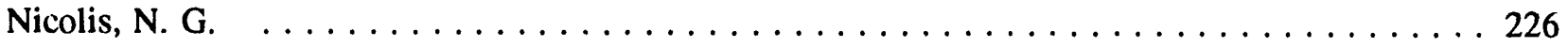

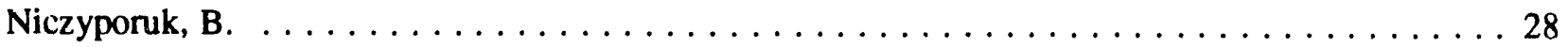

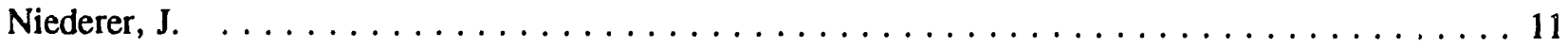

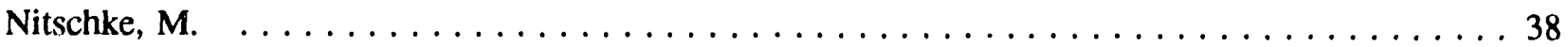

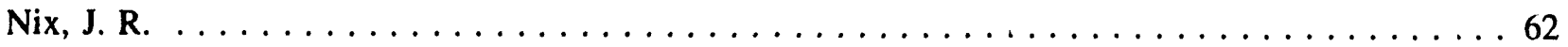

Nolen, J. A. $\ldots \ldots \ldots \ldots \ldots \ldots \ldots \ldots \ldots \ldots \ldots \ldots \ldots \ldots \ldots \ldots \ldots \ldots \ldots \ldots \ldots \ldots$

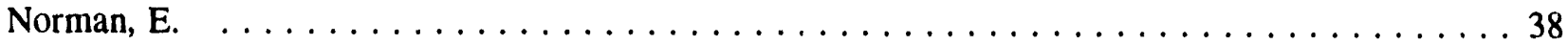

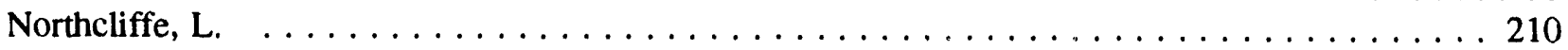

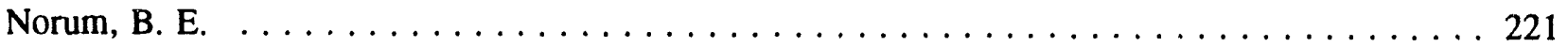

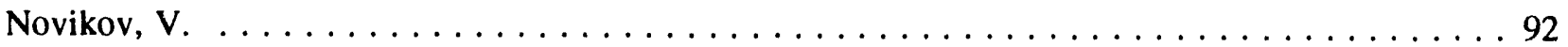

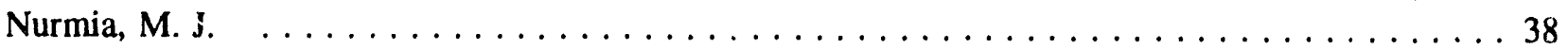

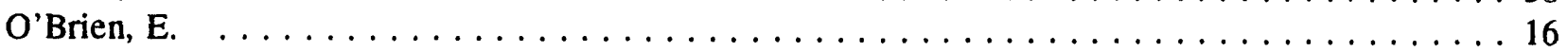

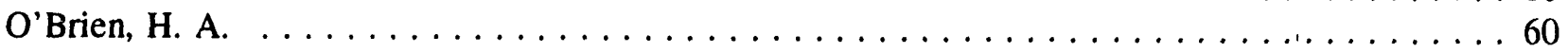

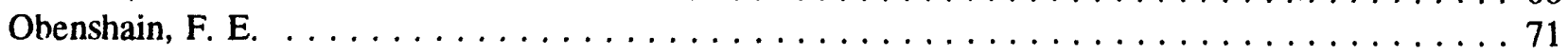

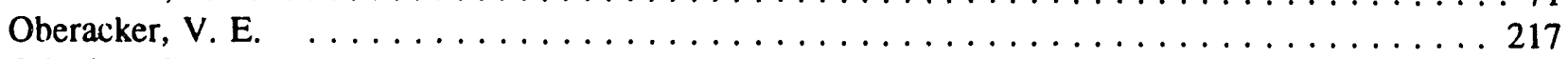

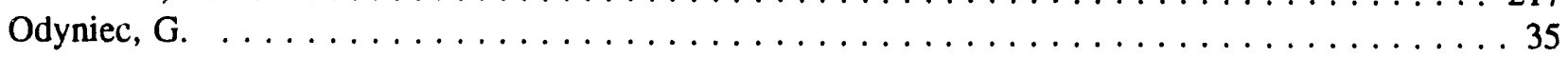

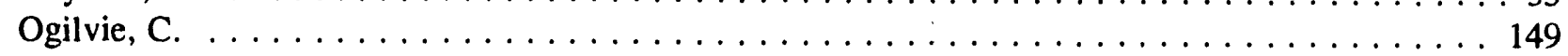

Oleszczuk, . $\ldots \ldots \ldots \ldots \ldots \ldots \ldots \ldots \ldots \ldots \ldots \ldots \ldots \ldots \ldots \ldots$

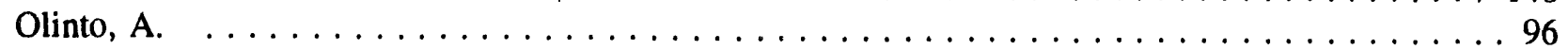

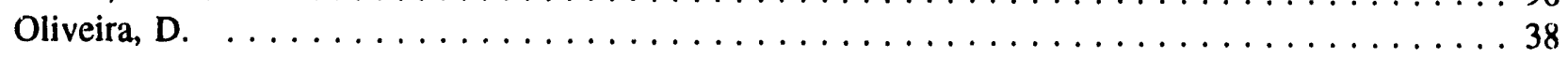

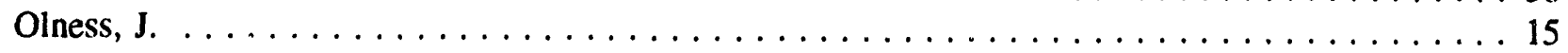




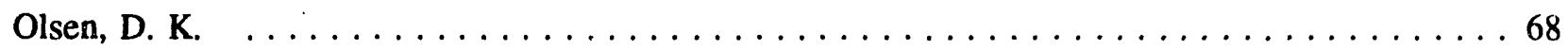

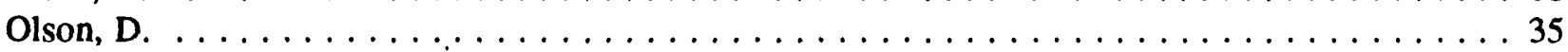

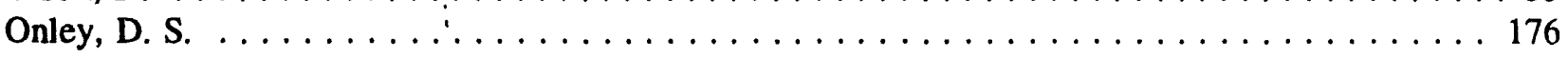

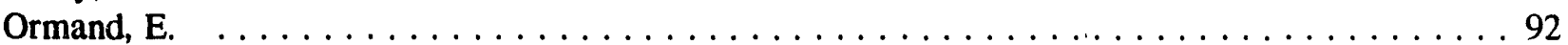

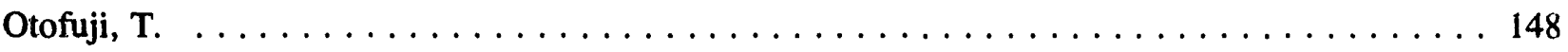

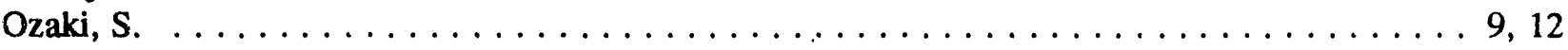

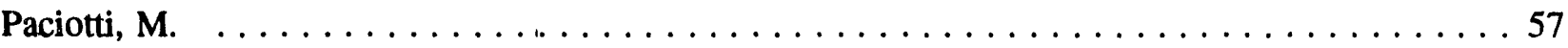

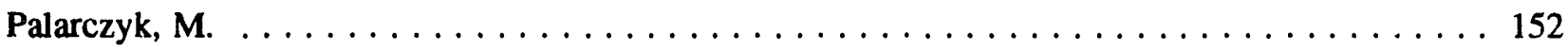

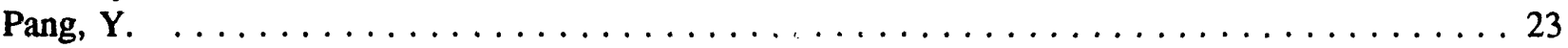

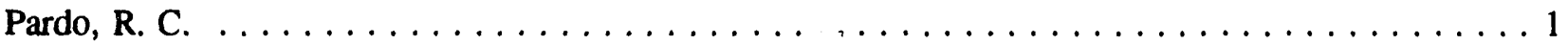

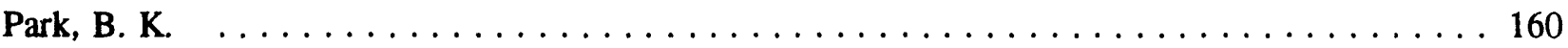

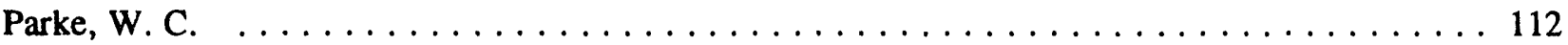

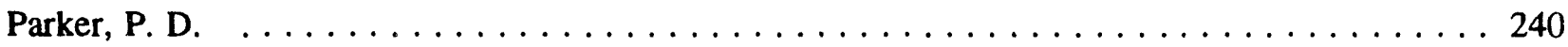

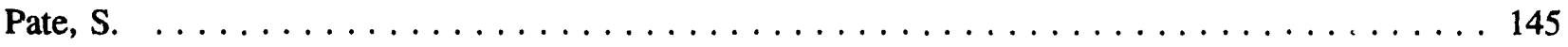

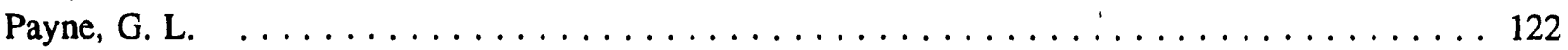

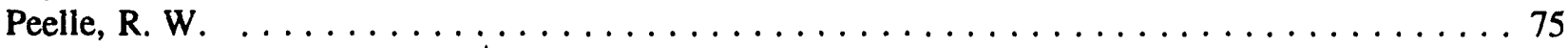

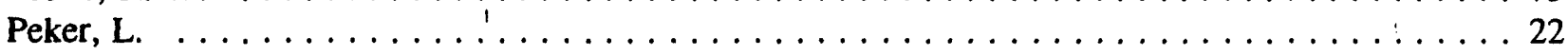

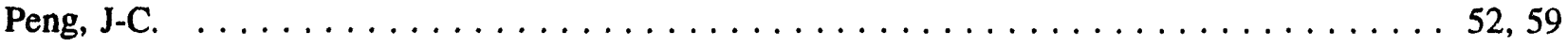

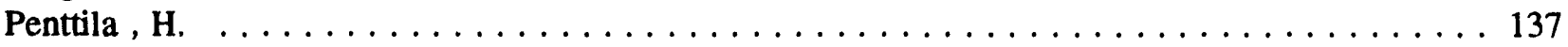

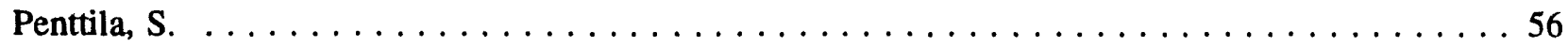

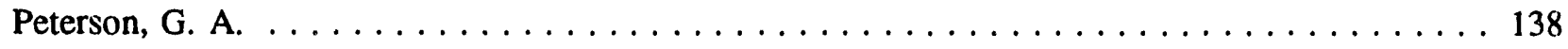

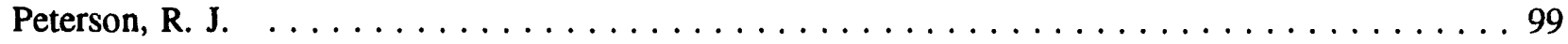

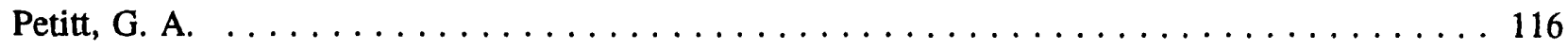

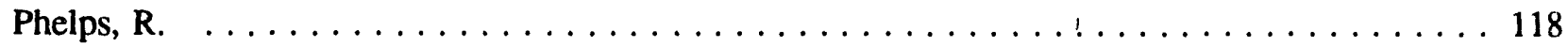

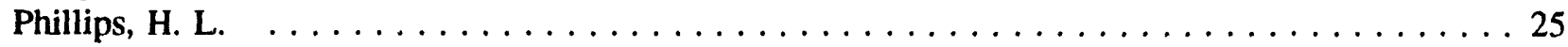

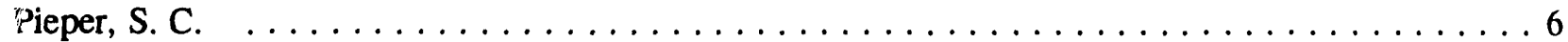

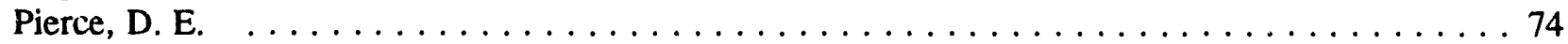

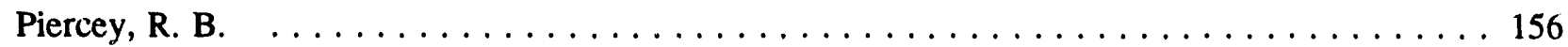

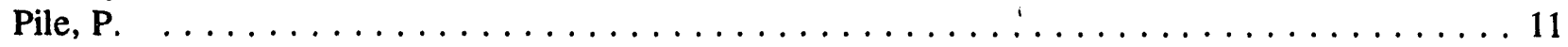

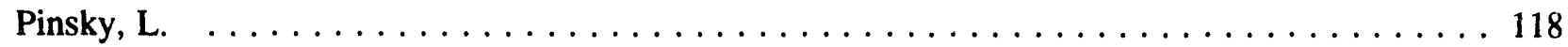

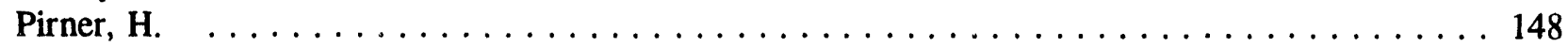

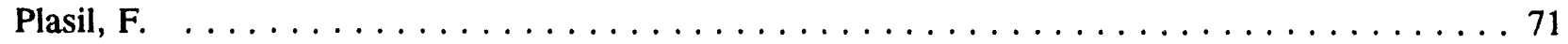

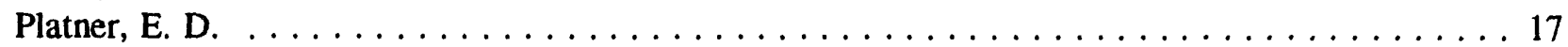

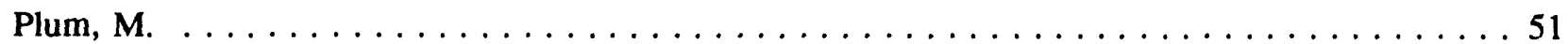

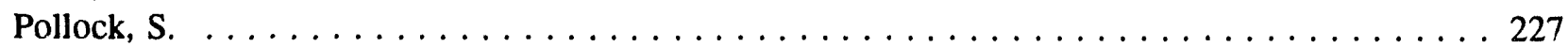

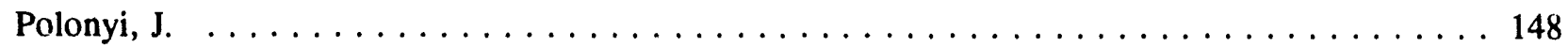

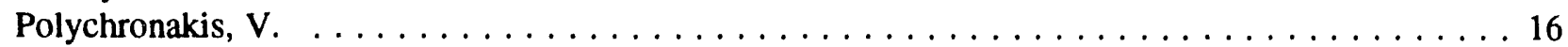

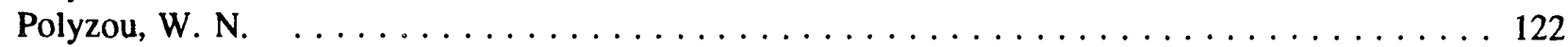

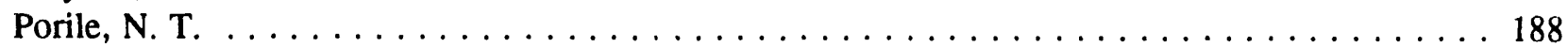

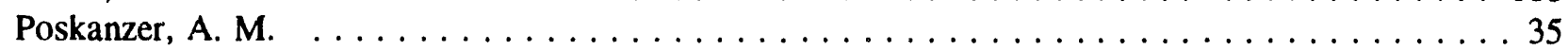

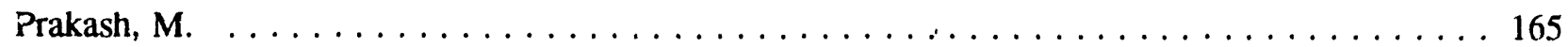

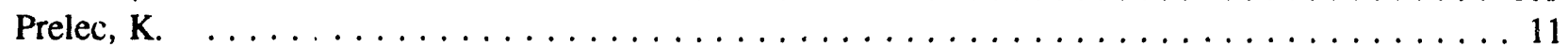

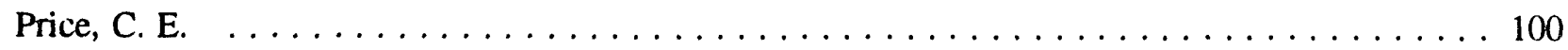

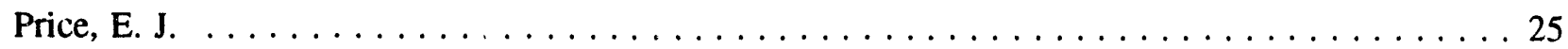

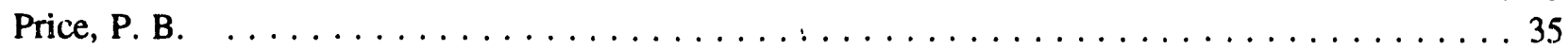

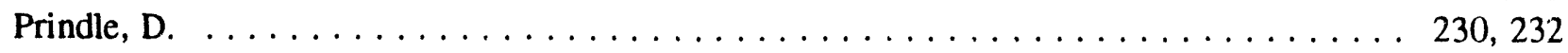

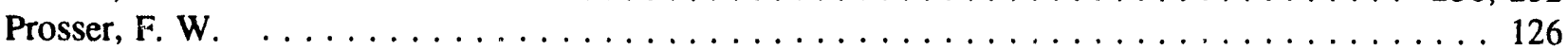

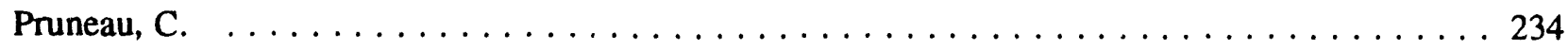

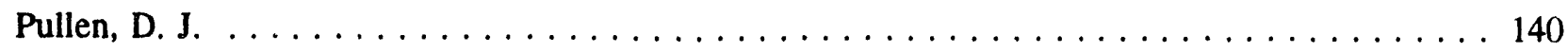




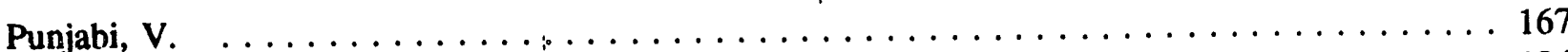

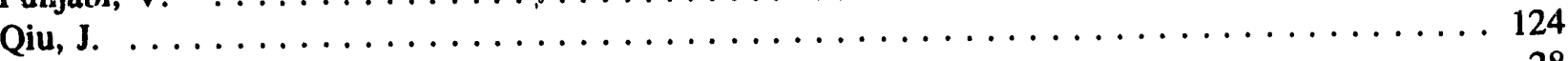

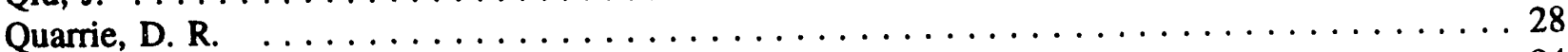

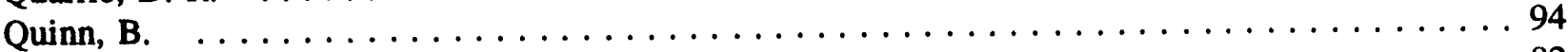

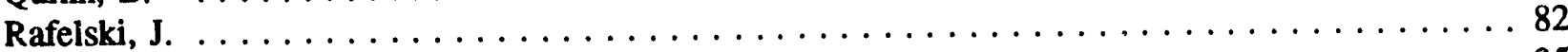

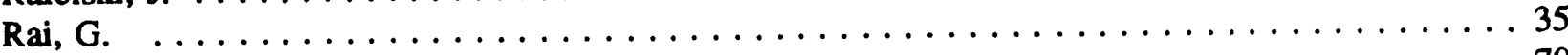

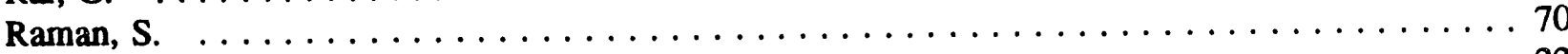

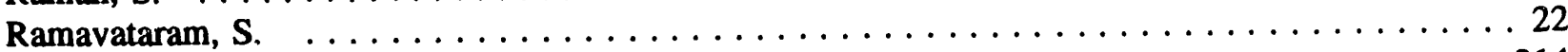

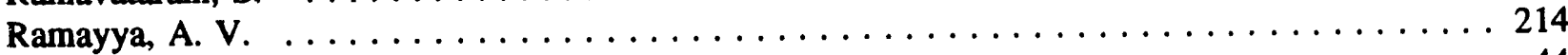

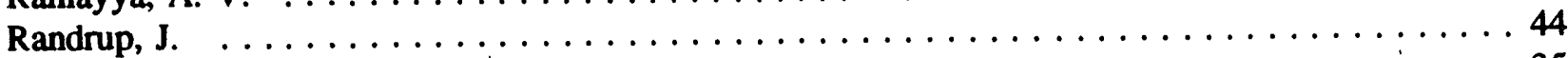

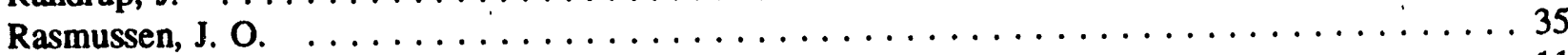

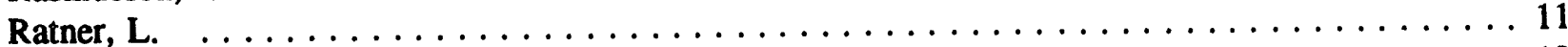

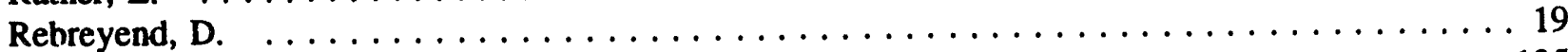

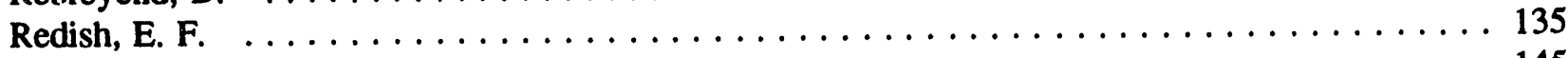

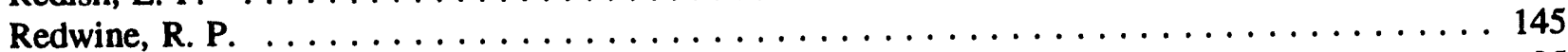

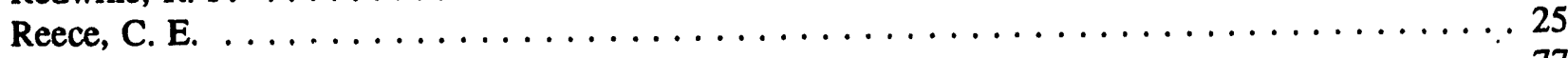

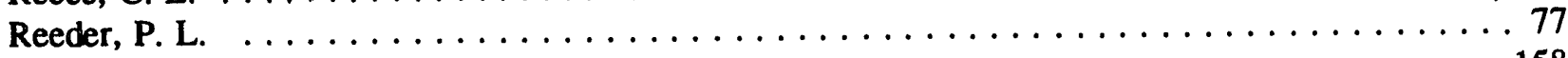

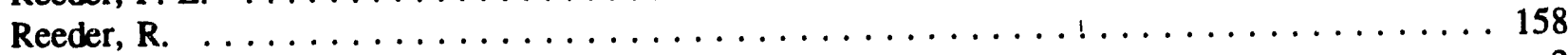

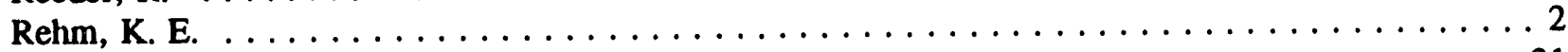

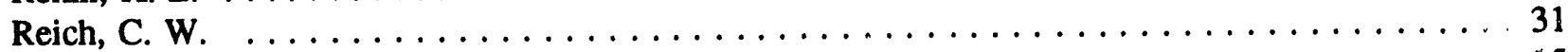

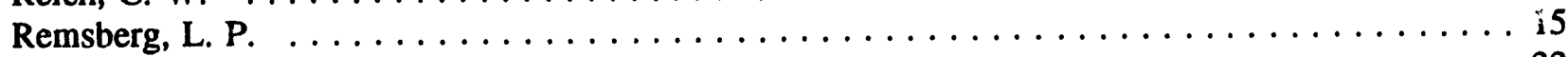

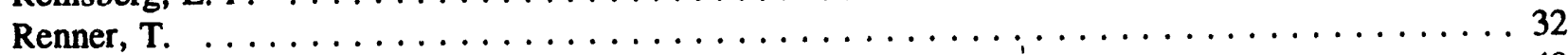

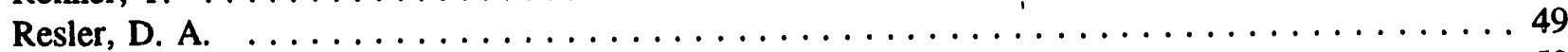

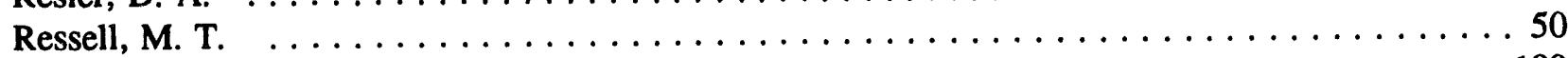

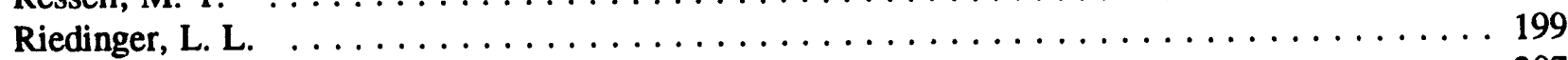

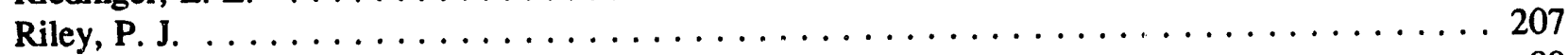

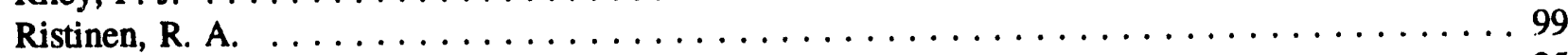

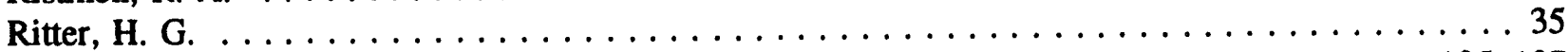

Roberson, N. R. . . . . . . . . . . . . . . . . . . . . . . . . . 105-107

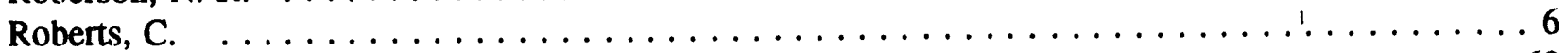

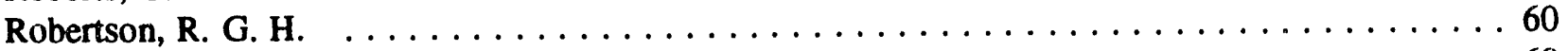

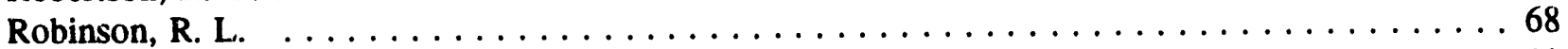

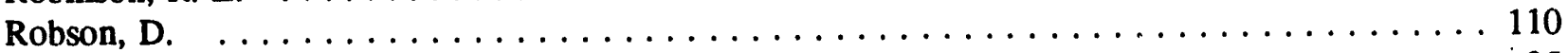

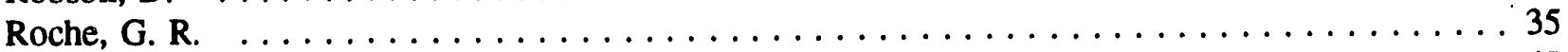

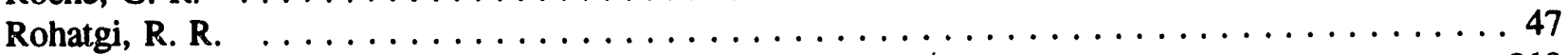

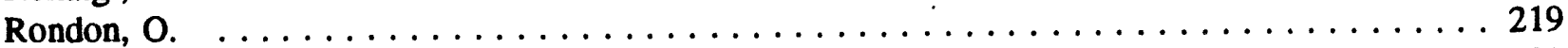

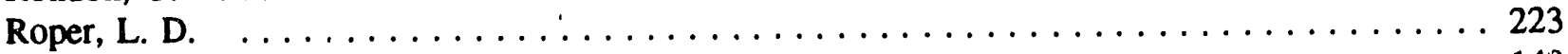

Rosenberg, L. . . . . . . . . . . . . . . . . . . . . . . . . . 149

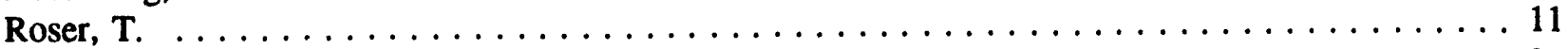

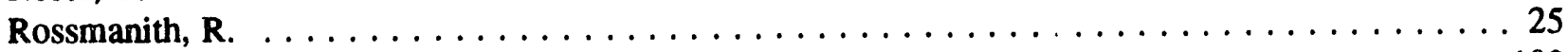

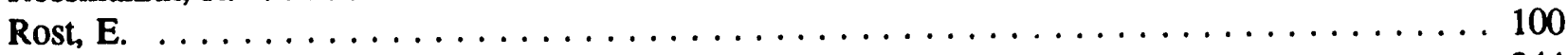

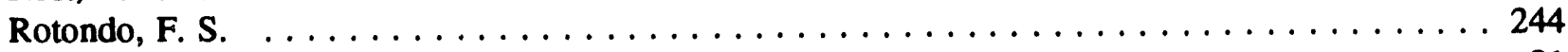

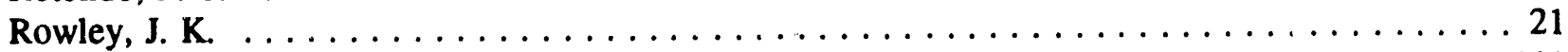

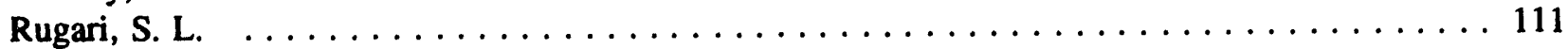

Ryan, J. . . . . . . . . . . . . . . . . . . . . . . . . . 149

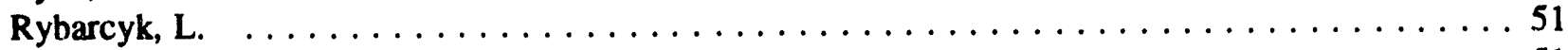

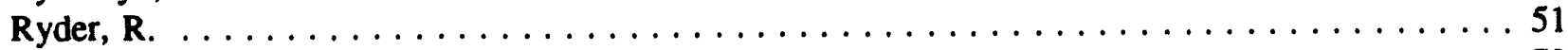

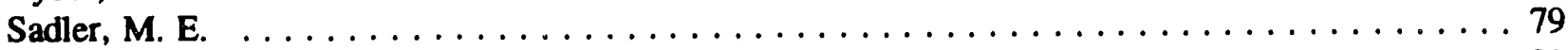

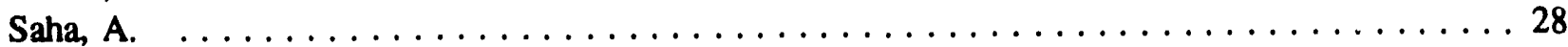

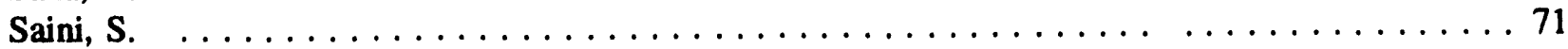




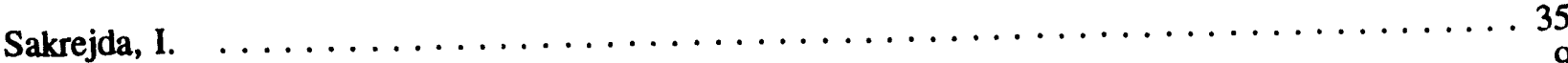

Samios, N. P.

Sandberg, $V$.

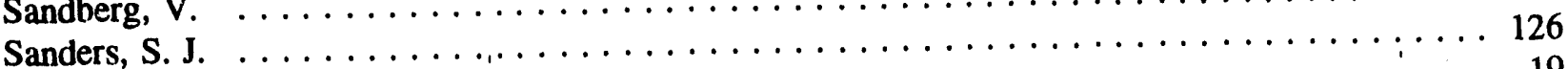

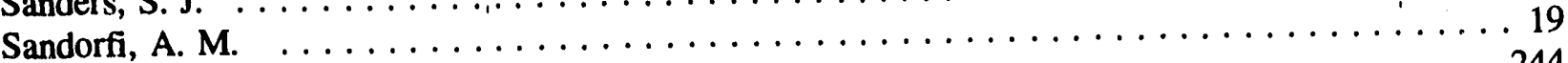

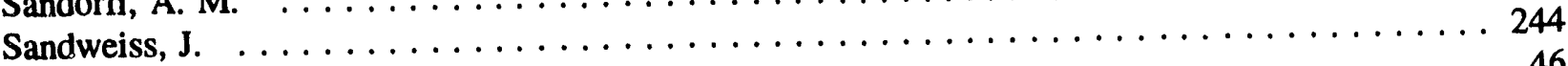

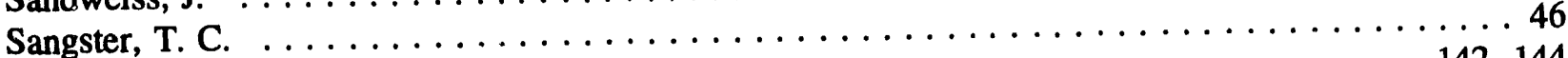

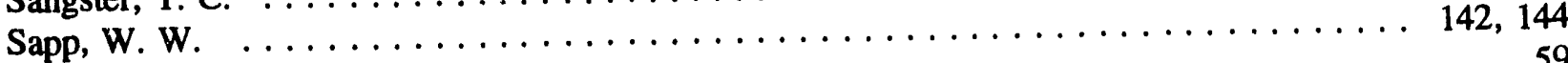

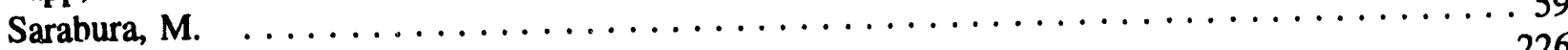

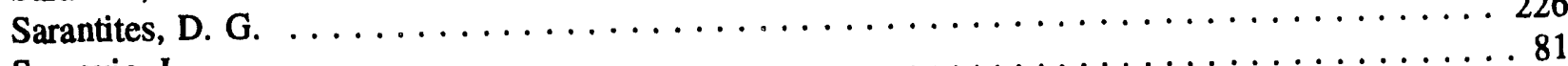

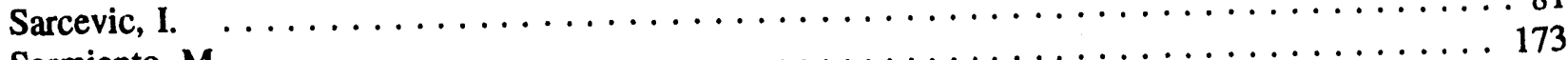

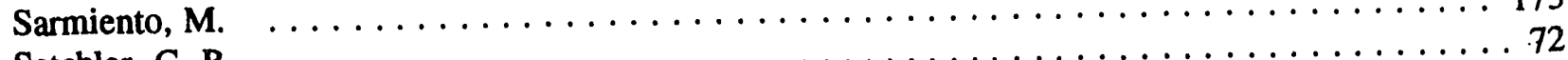

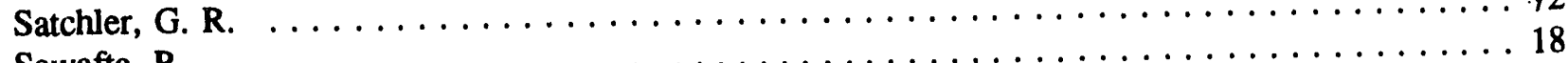

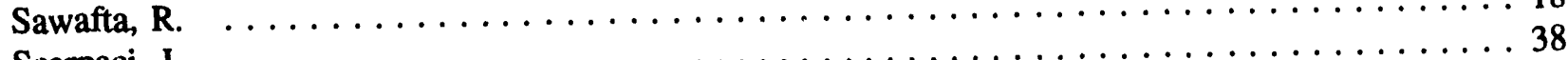

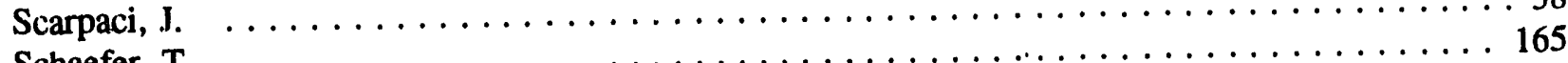

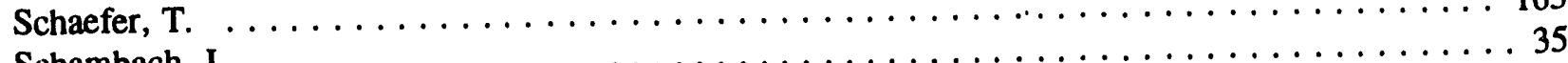

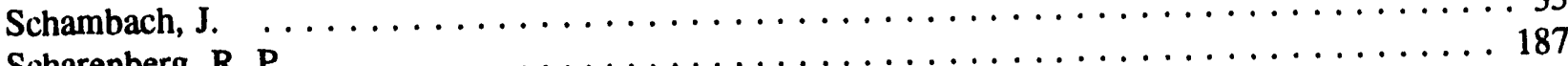

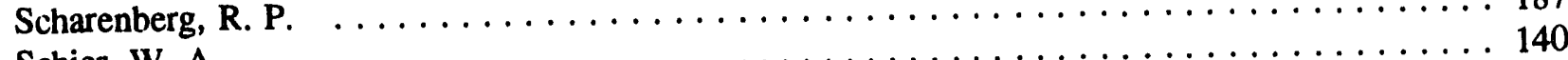

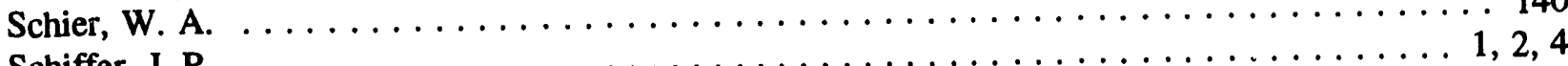

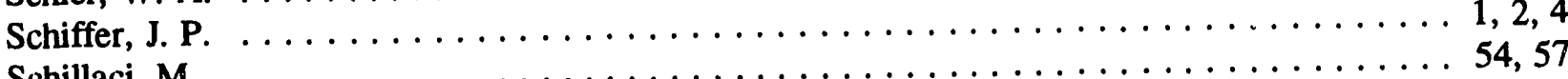

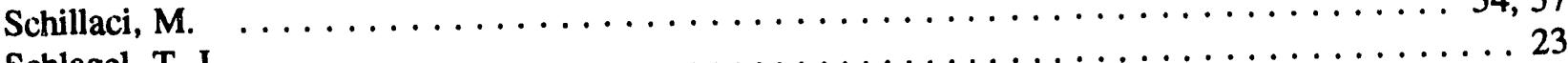

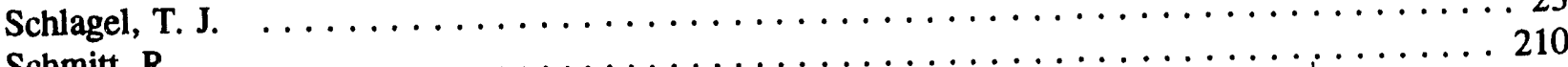

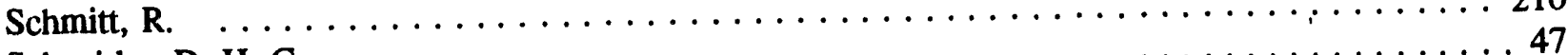

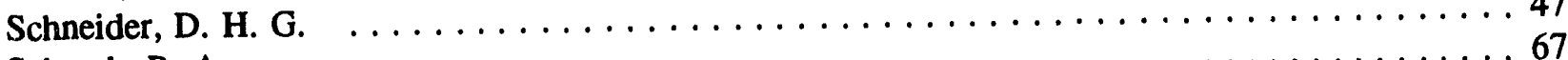

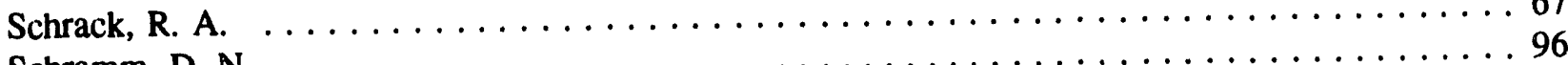

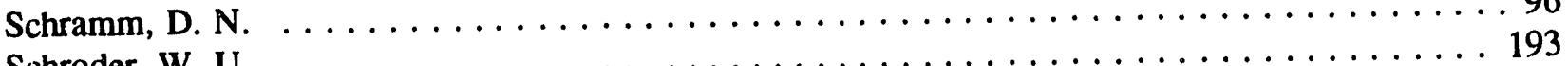

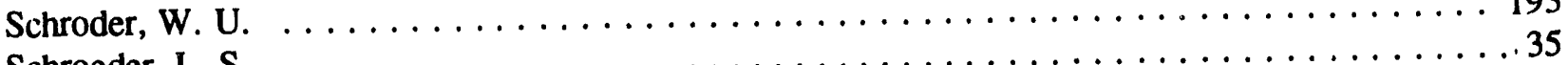

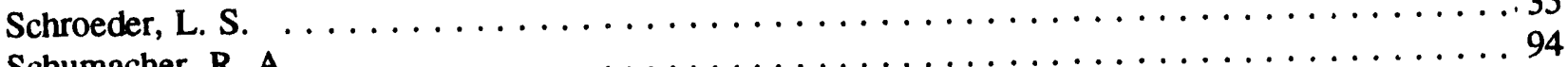

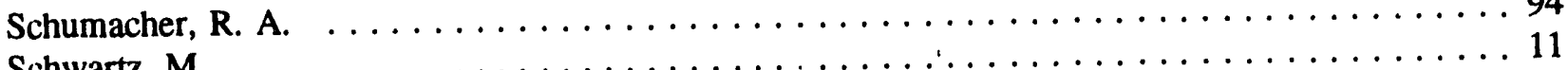

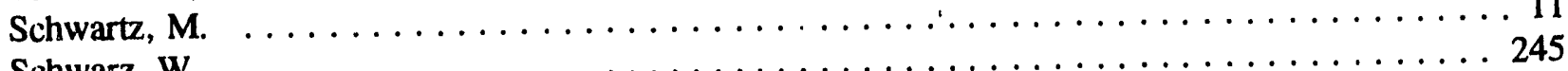

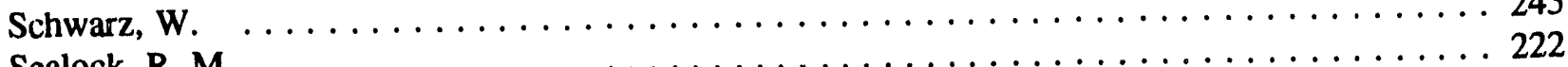

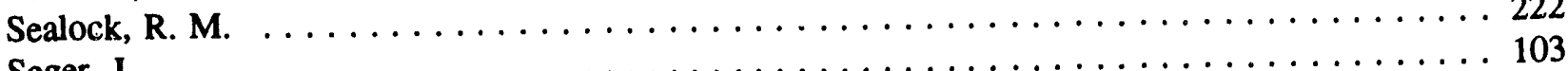

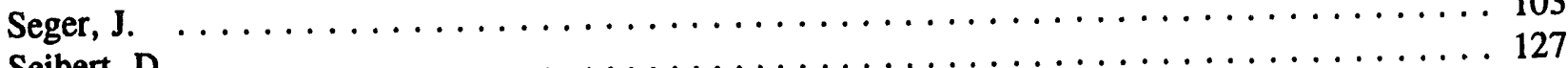

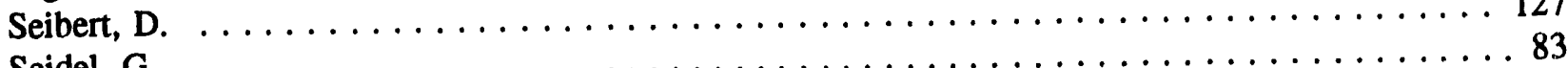

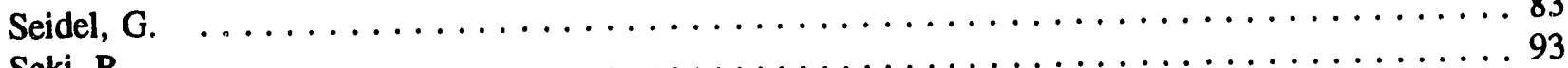

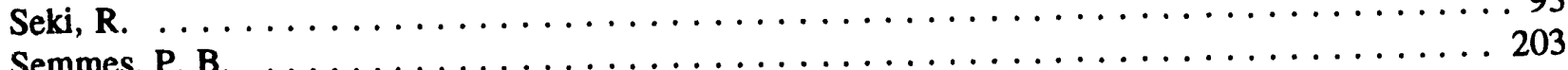

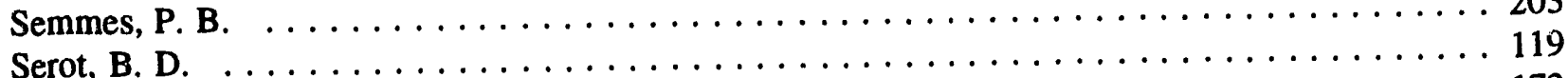

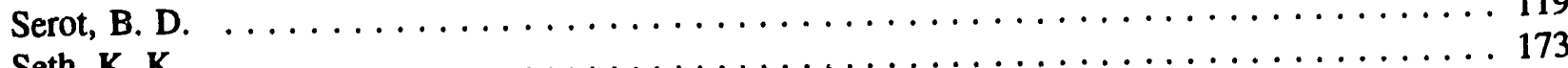

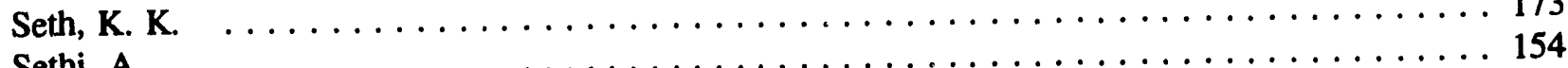

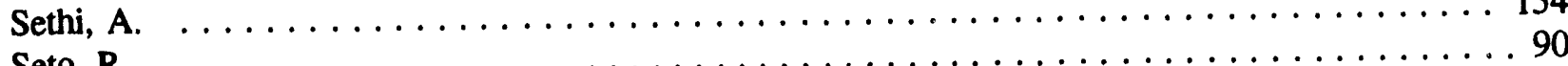

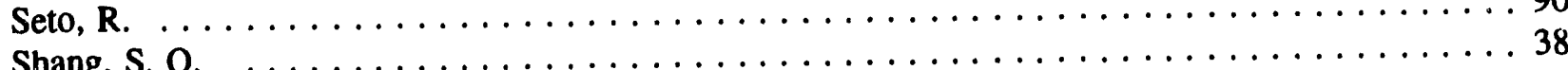

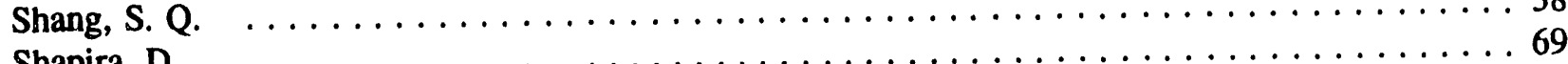

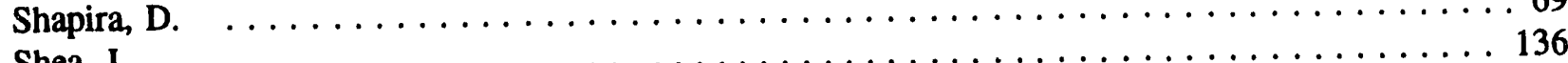

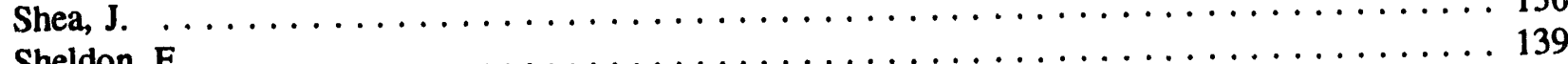

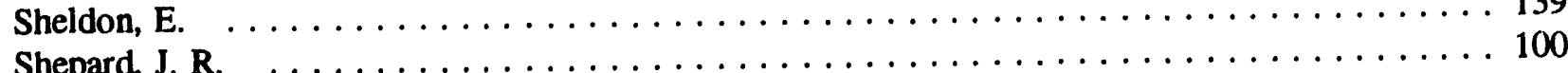

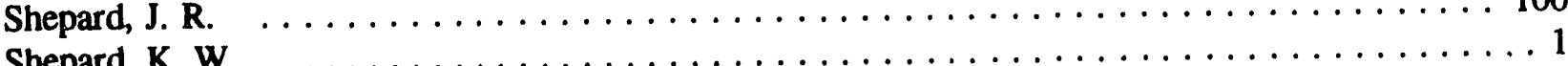

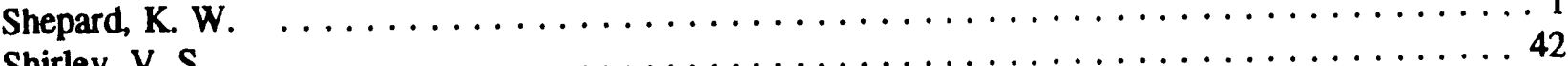

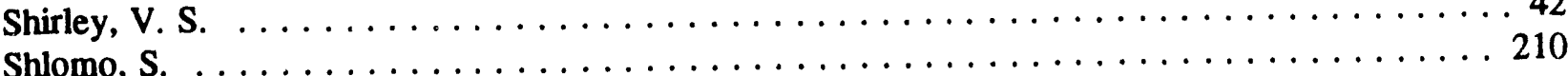




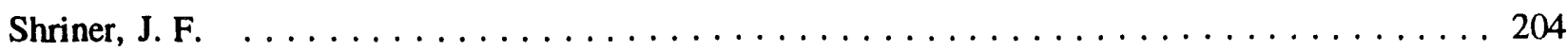

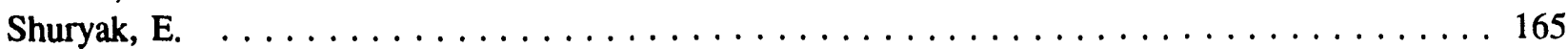

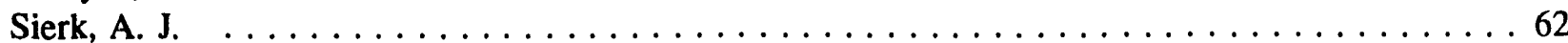

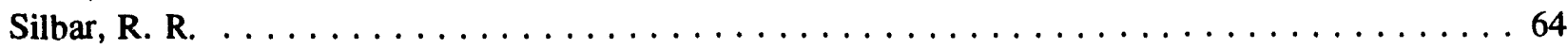

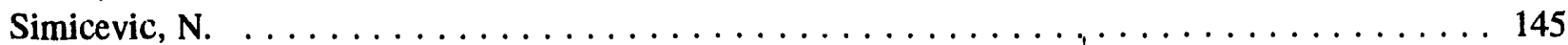

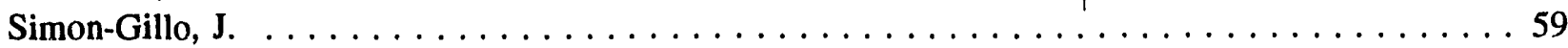

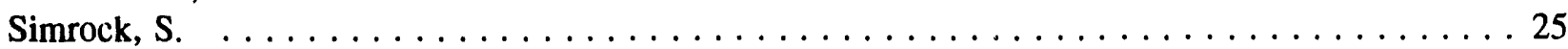

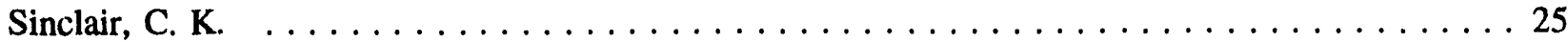

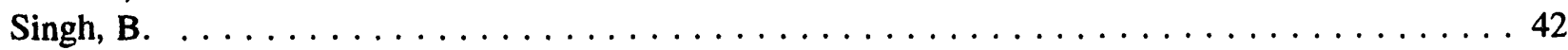

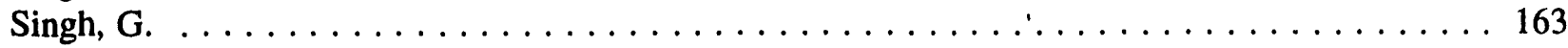

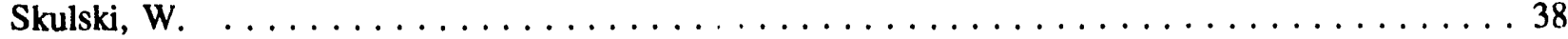

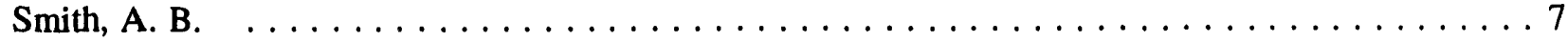

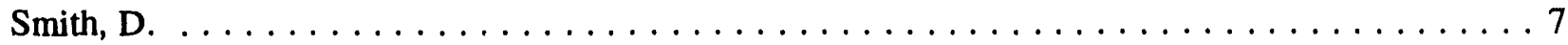

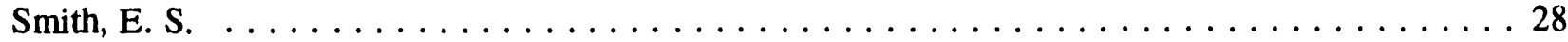

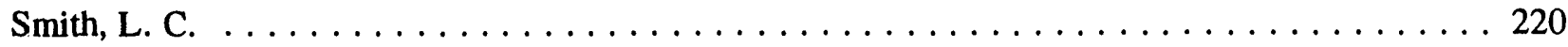

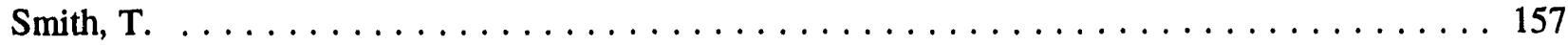

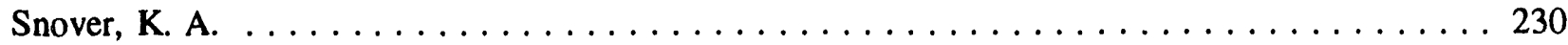

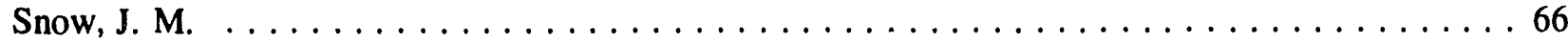

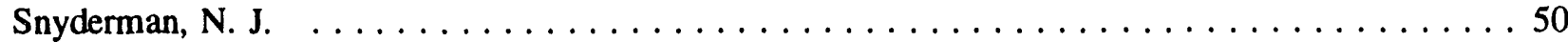

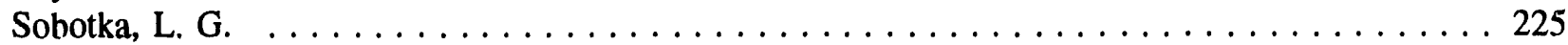

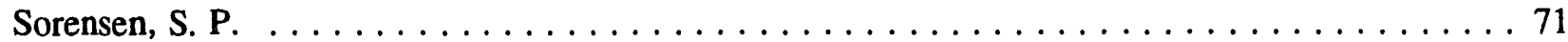

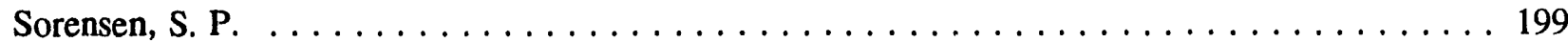

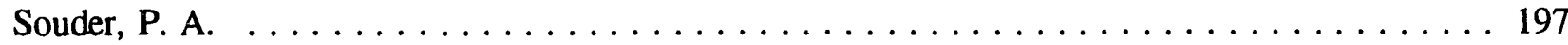

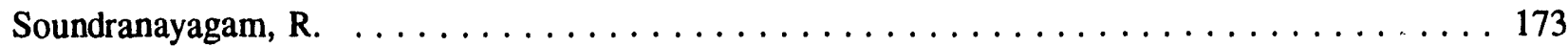

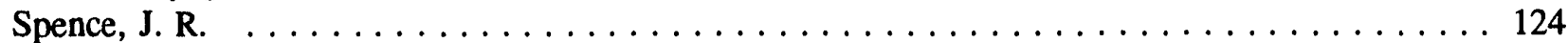

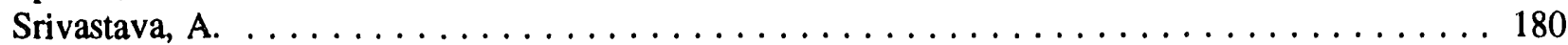

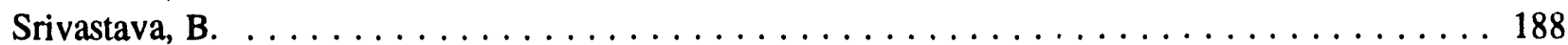

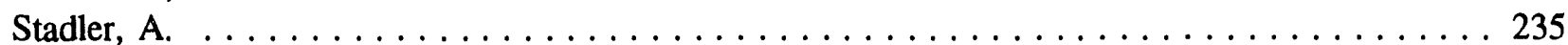

Stancu, I. . . . . . . . . . . . . . . . . . . . . . . . . . . . . . . . . . 190

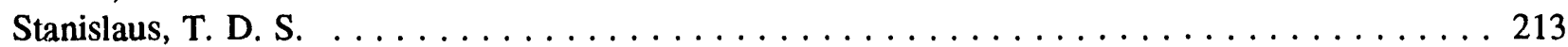

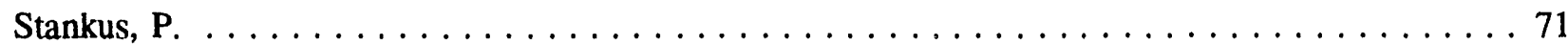

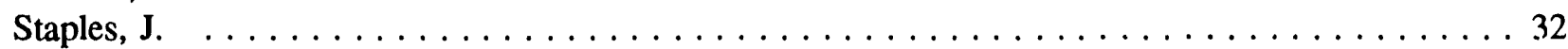

Starodubsky, V. . . . . . . . . . . . . . . . . . . . . . . . . . 154

Steadman, S. . . . . . . . . . . . . . . . . . . . . . . . . . . . 149

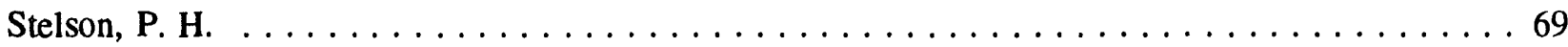

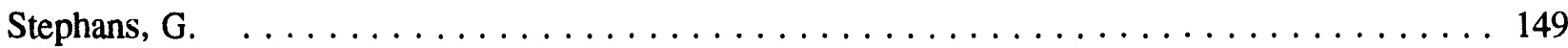

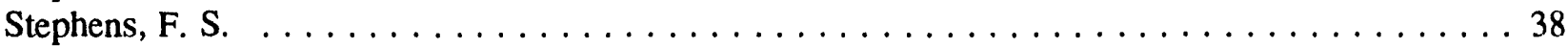

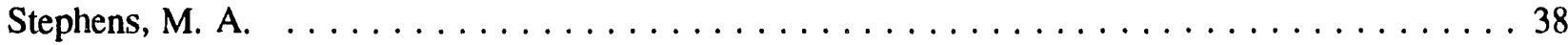

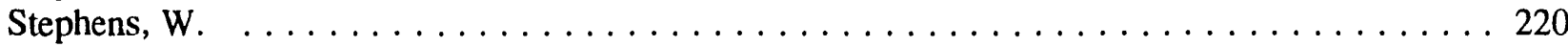

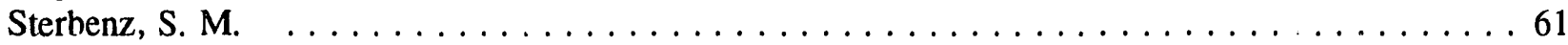

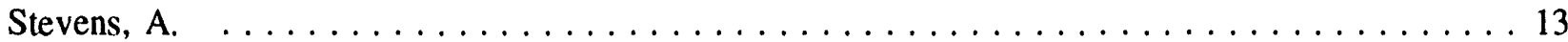

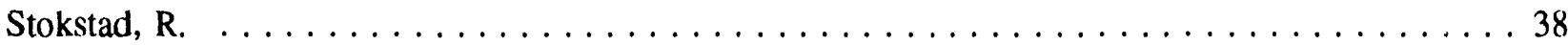

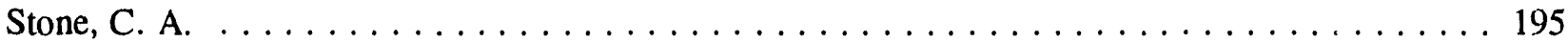

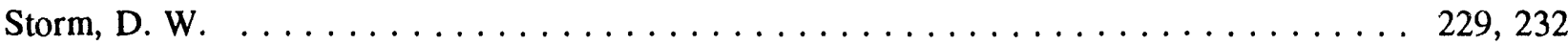

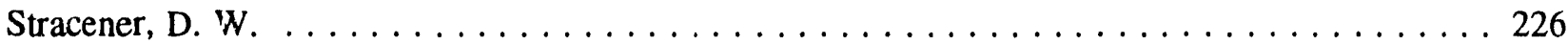

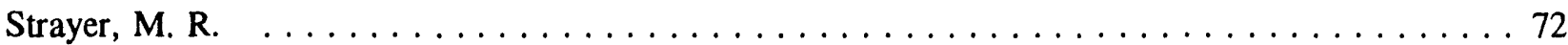

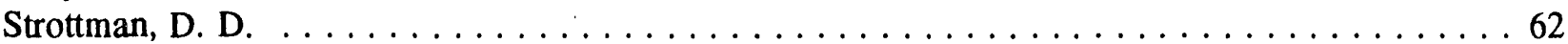

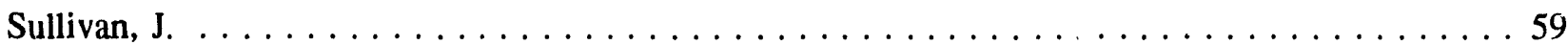

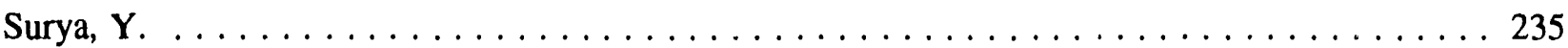

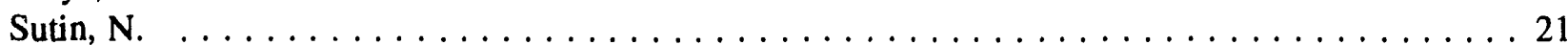




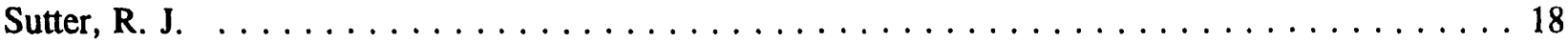

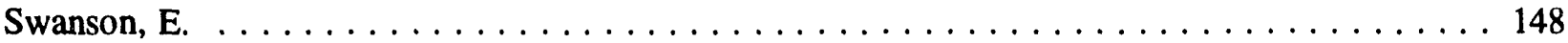

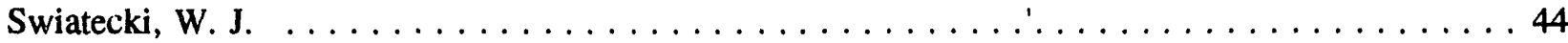

Symons, T. J. M. $\ldots \ldots \ldots \ldots \ldots \ldots \ldots \ldots \ldots \ldots \ldots \ldots \ldots \ldots \ldots \ldots \ldots \ldots \ldots \ldots \ldots \ldots \ldots \ldots \ldots \ldots, 35,38,42$

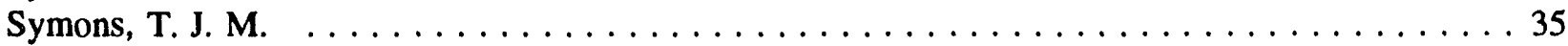

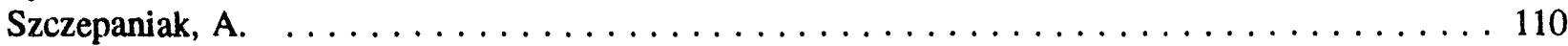

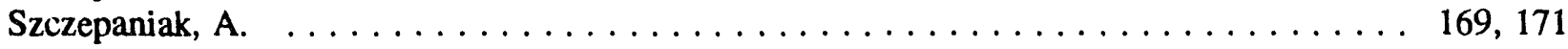

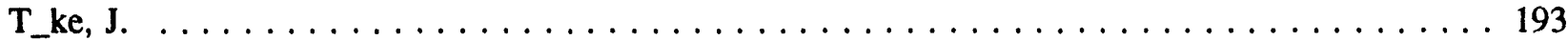

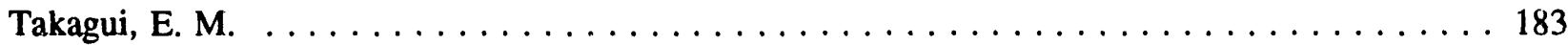

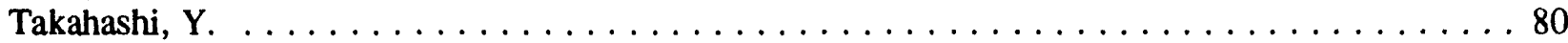

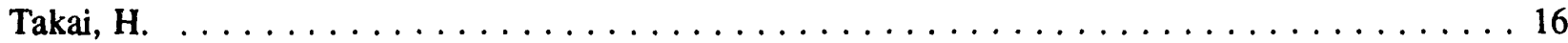

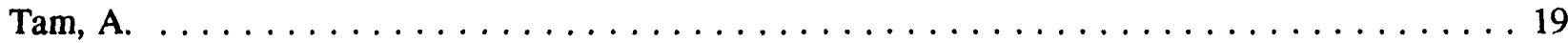

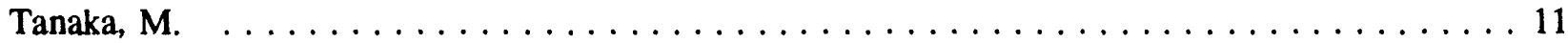

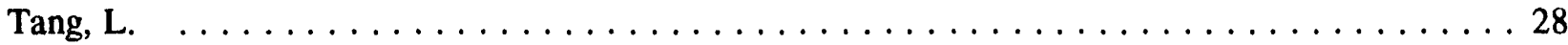

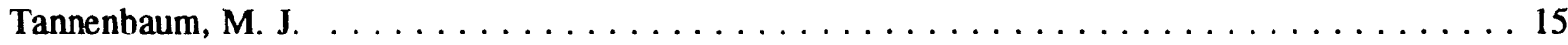

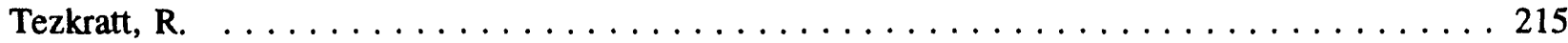

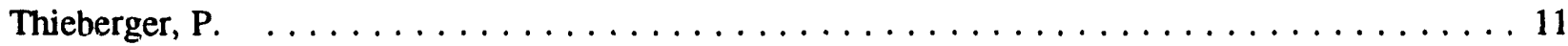

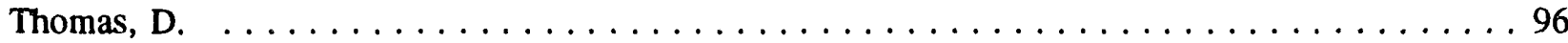

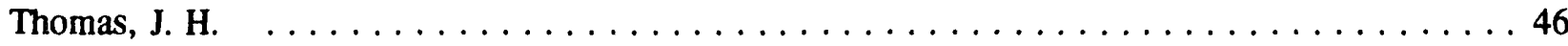

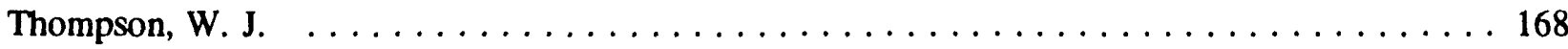

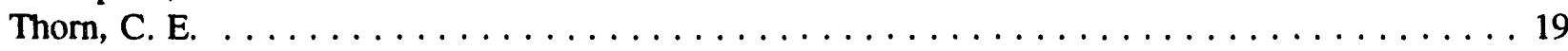

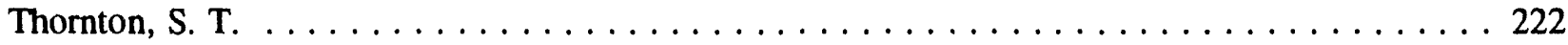

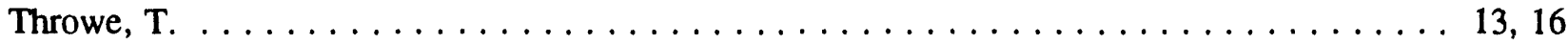

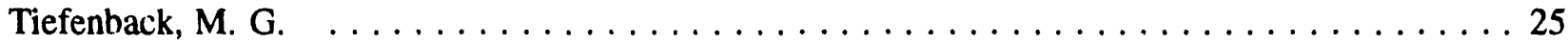

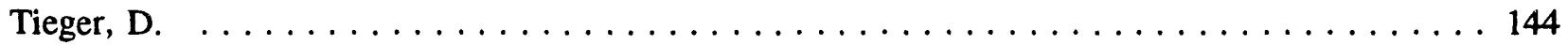

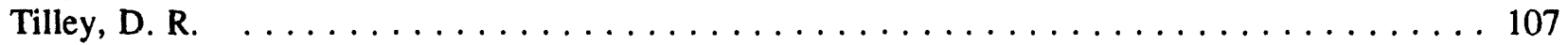

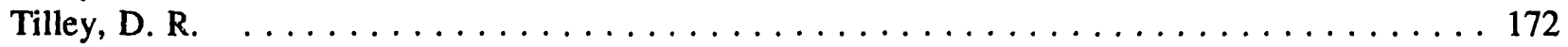

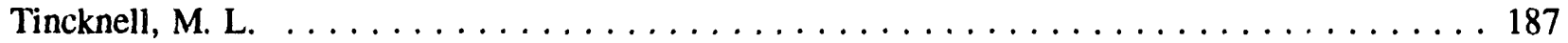

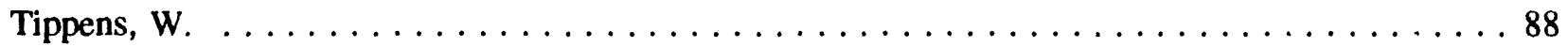

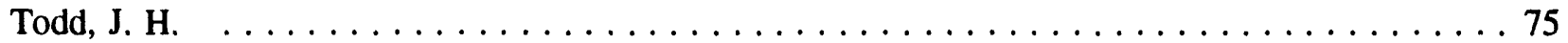

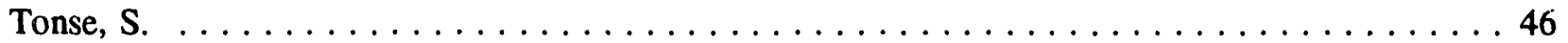

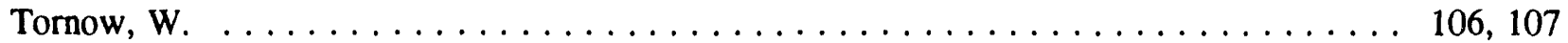

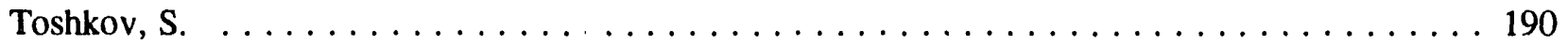

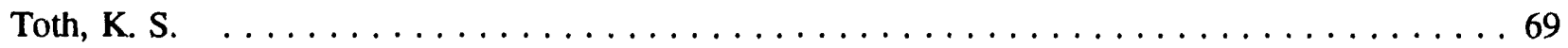

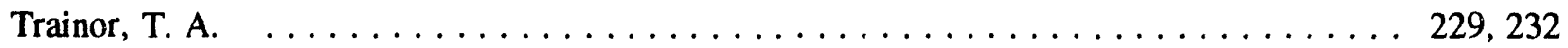

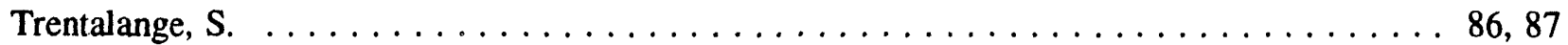

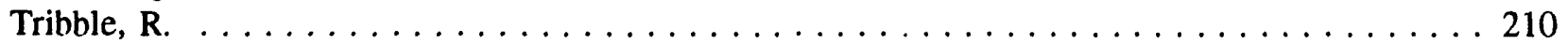

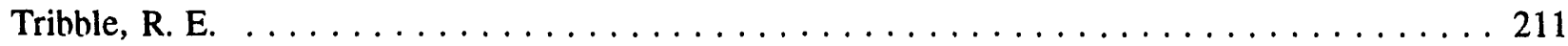

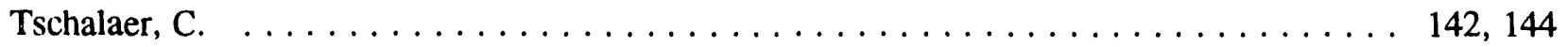

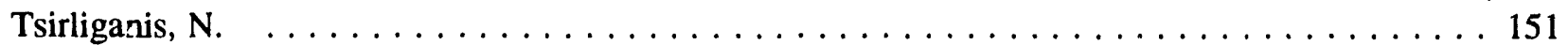

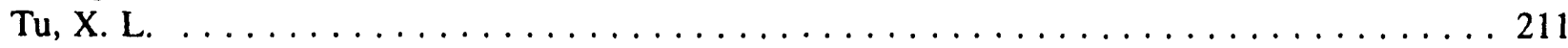

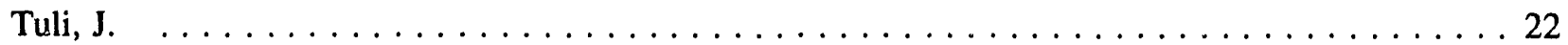

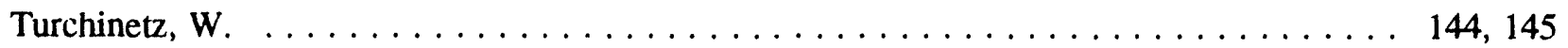

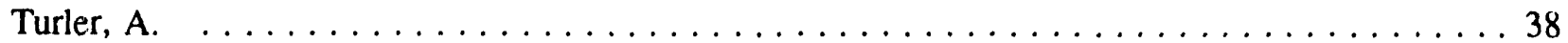

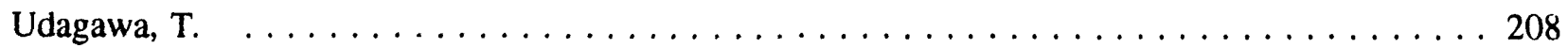

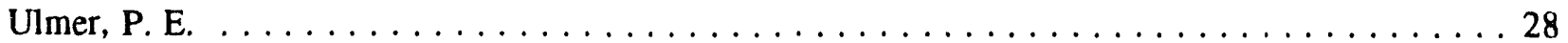

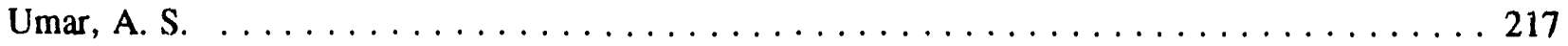

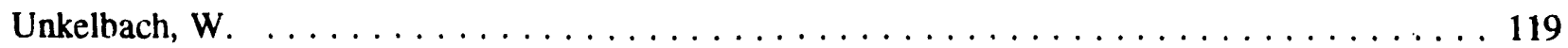

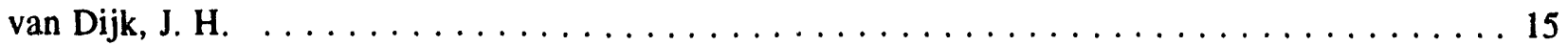


van Dyck, $O$. 51,57

van Hecke, $H$.

Van Dalen, G. J

Van Verst, $S$.

vanAsselt, W.

Vandenbosch, $\mathbf{R}$.

Vardaci, E.

Vary, J. P.

Verbaarschot, J.

Verdier, R.

Videbaek, F.

Vieira, D.

Vieira, D. J.

Vineyard, M. F.

Viola, V. E.

Vlahovic, B.

Vogel, P.

Wada, $\mathbf{R}$.

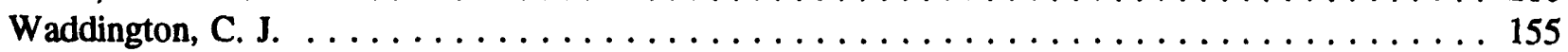

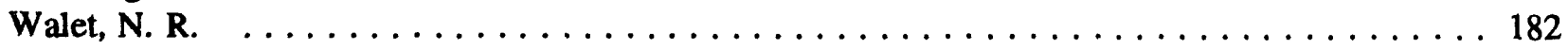

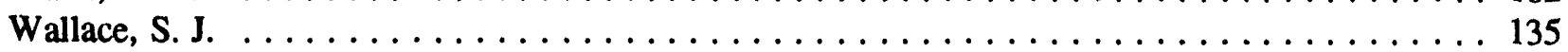

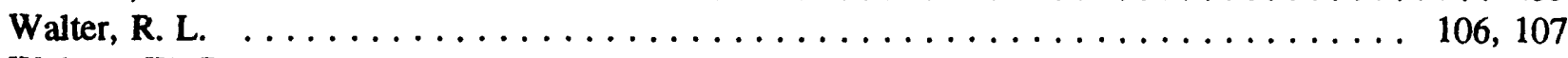

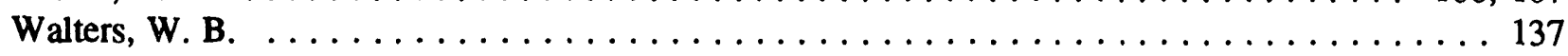

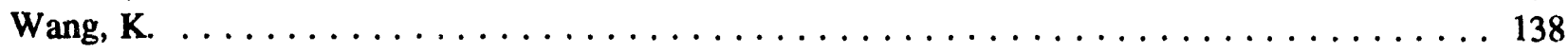

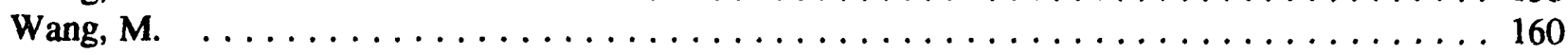

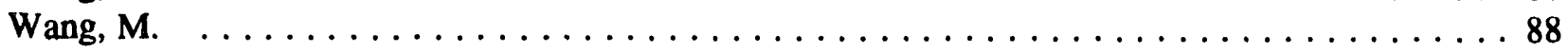

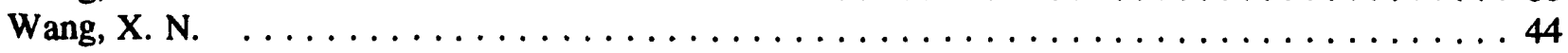

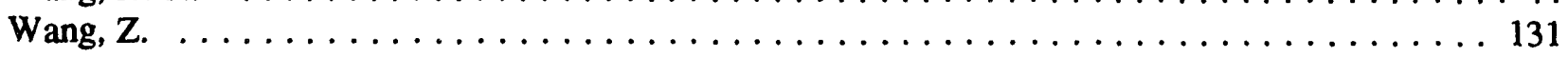

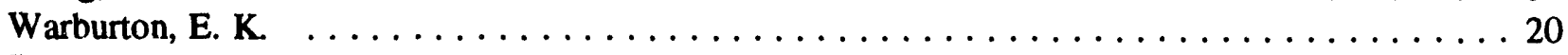

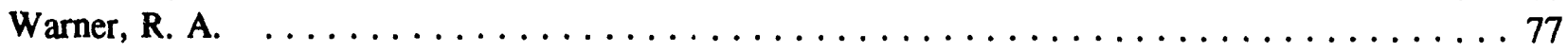

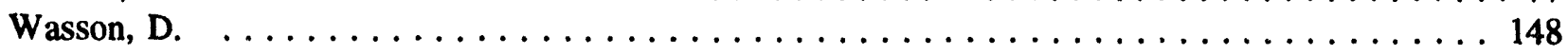

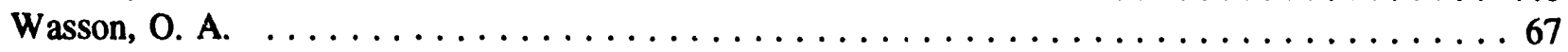

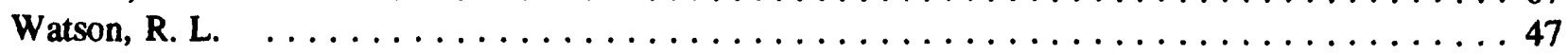

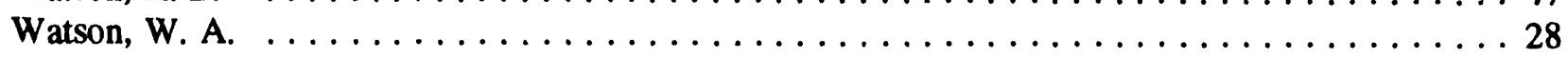

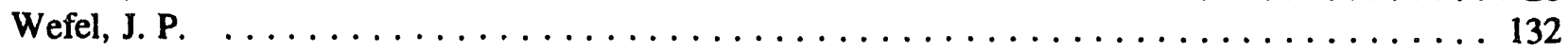

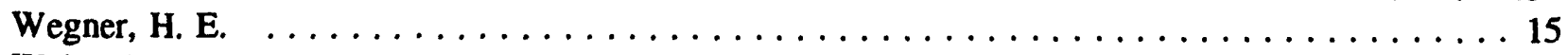

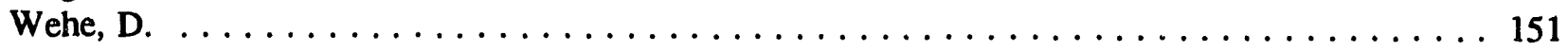

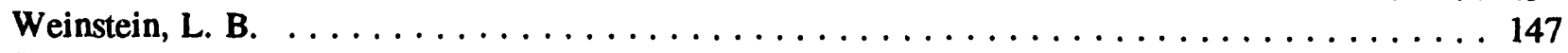

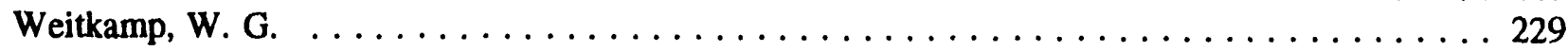

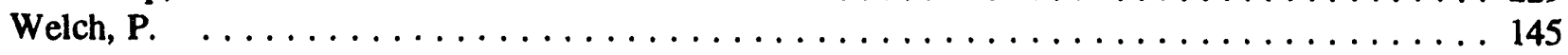

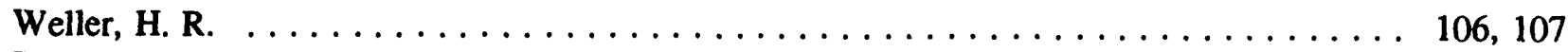

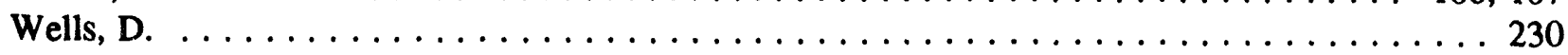

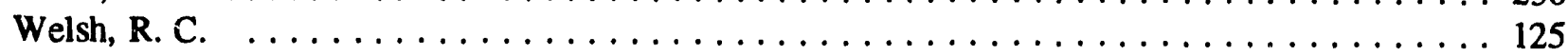

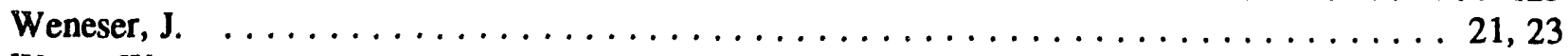

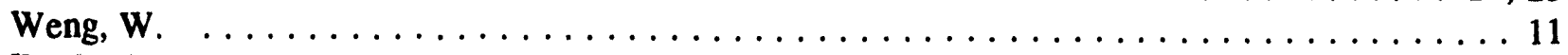

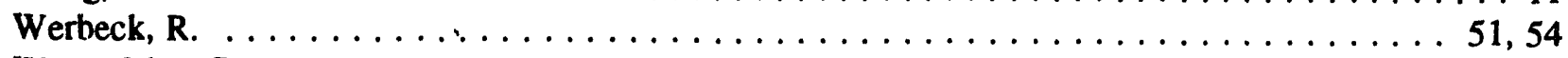

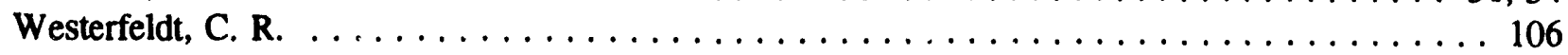

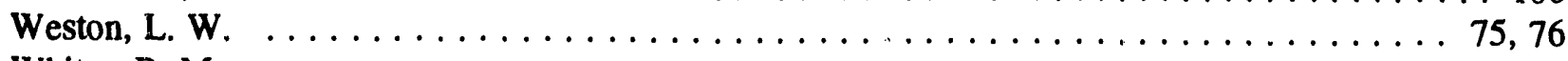

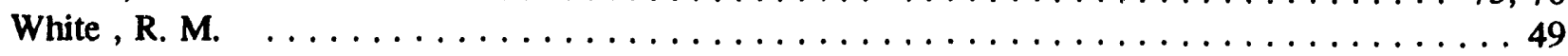


White, D. H.

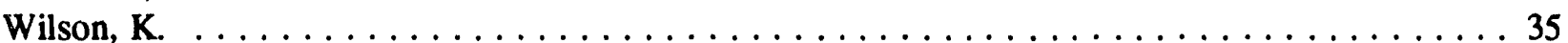

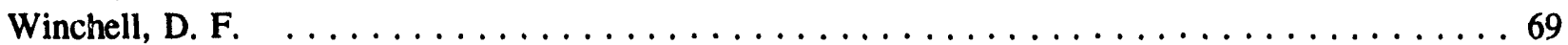

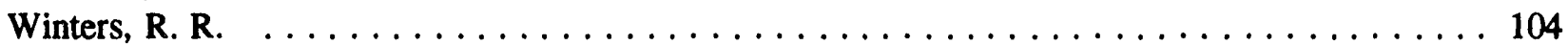

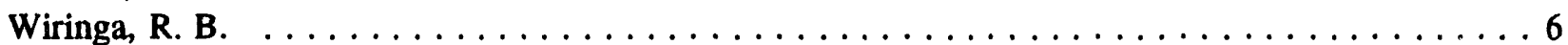

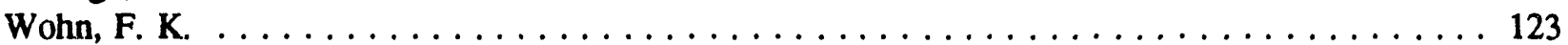

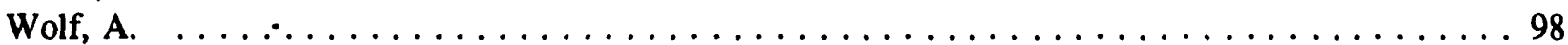

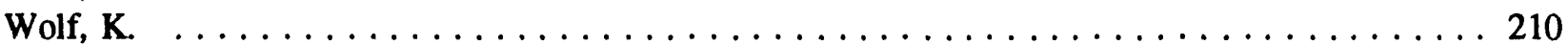

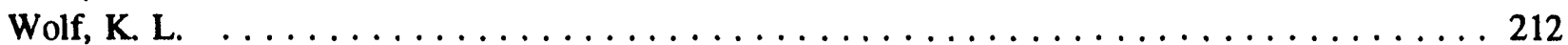

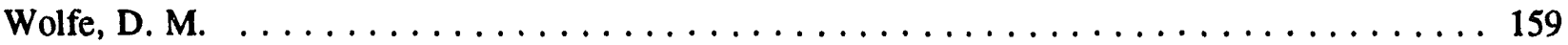

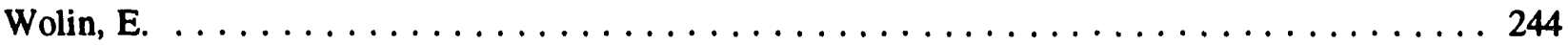

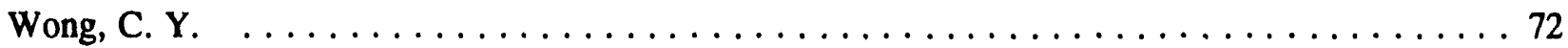

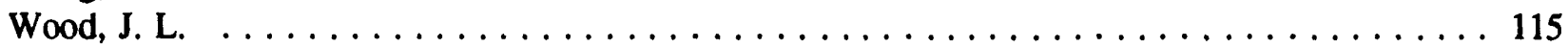

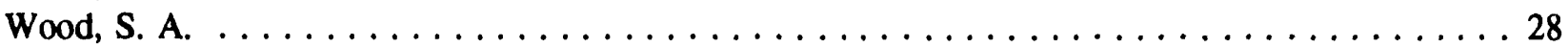

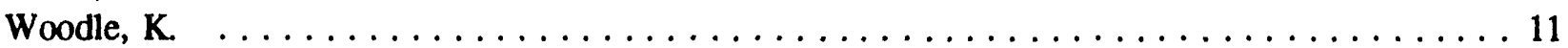

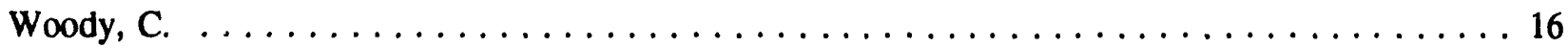

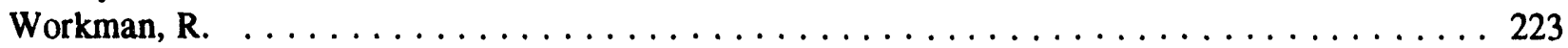

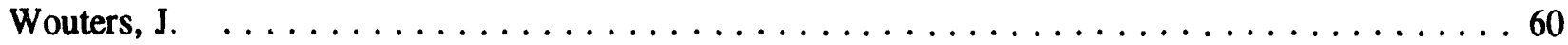

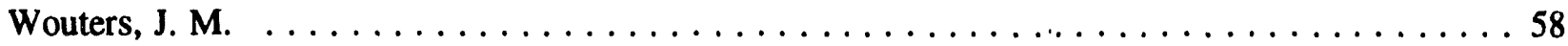

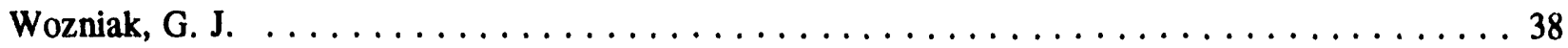

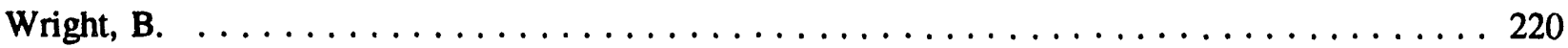

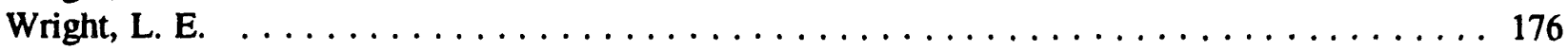

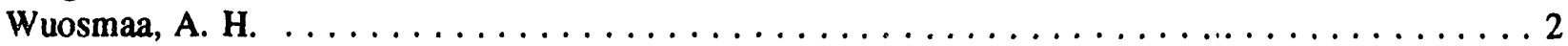

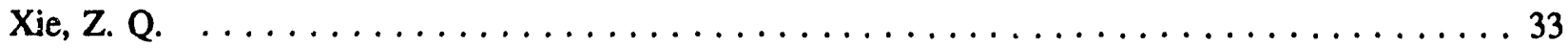

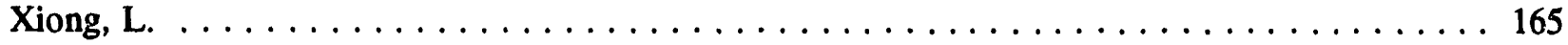

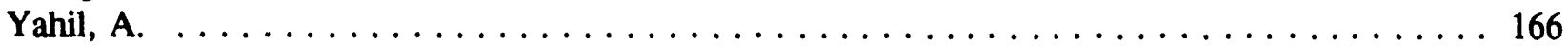

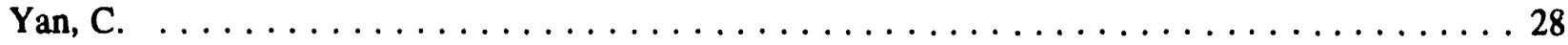

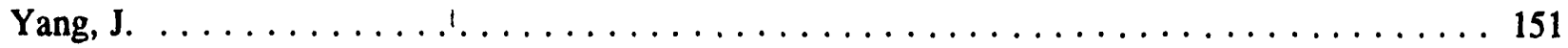

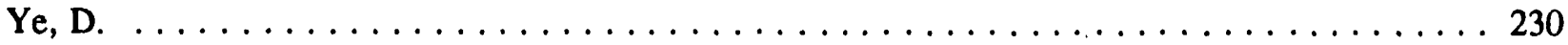

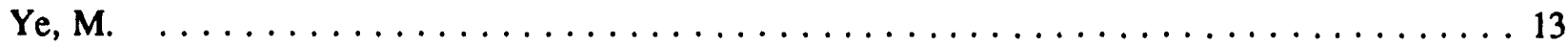

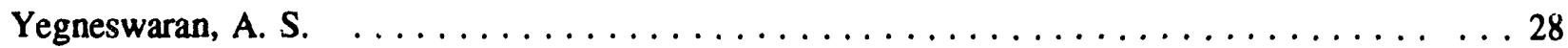

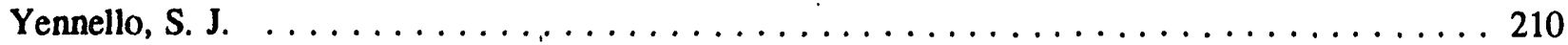

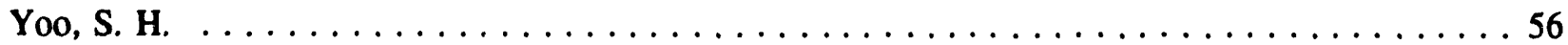

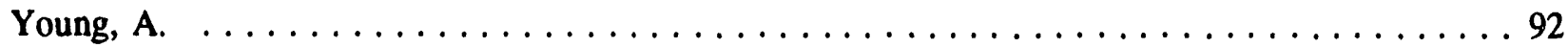

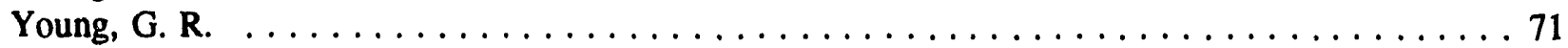

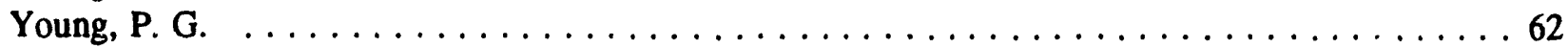




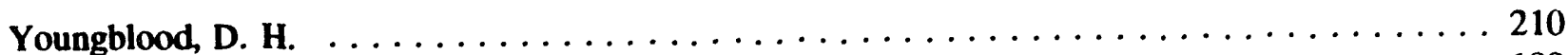

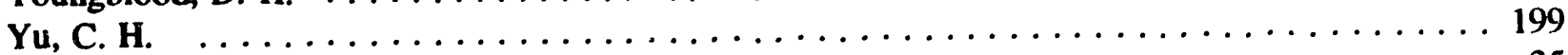

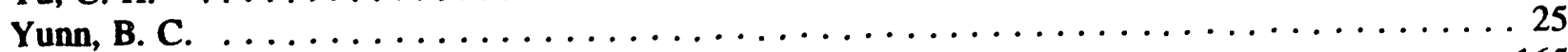

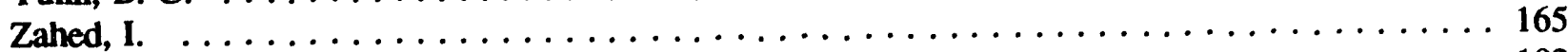

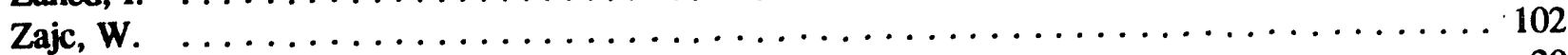

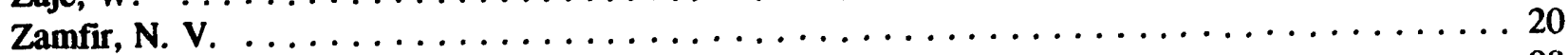

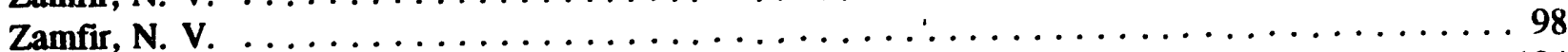

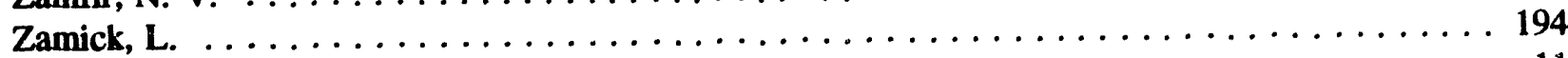

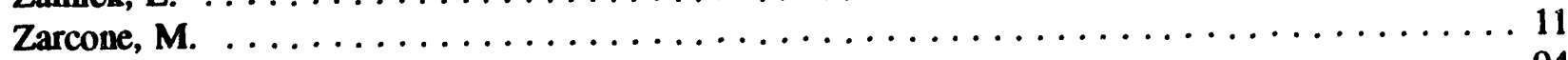

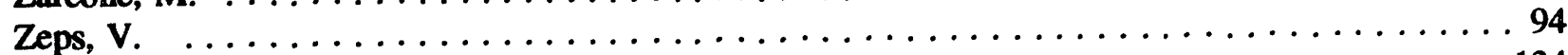

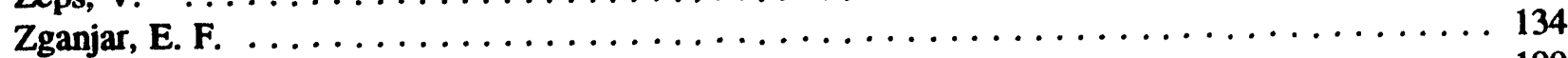

Zhang, J. ...................................... 199

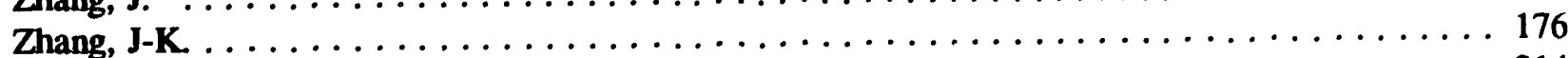

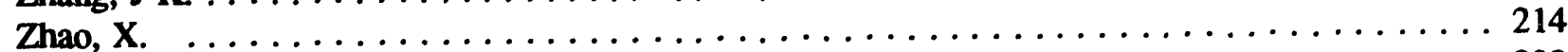

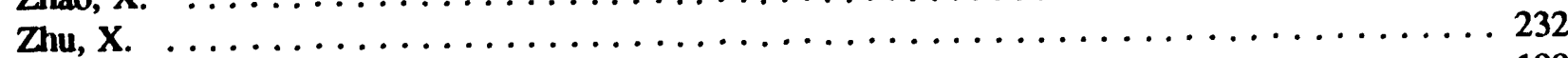

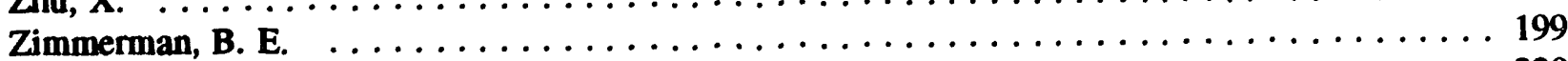

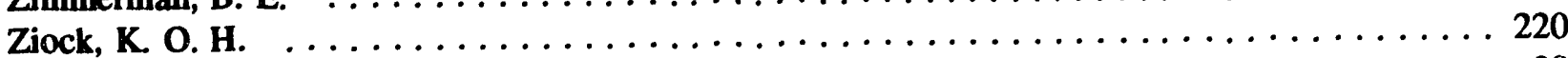

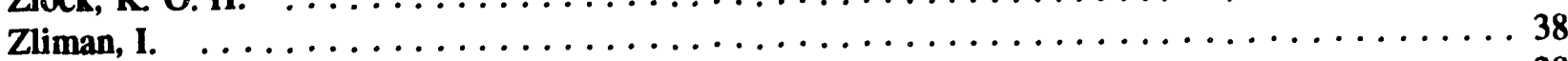

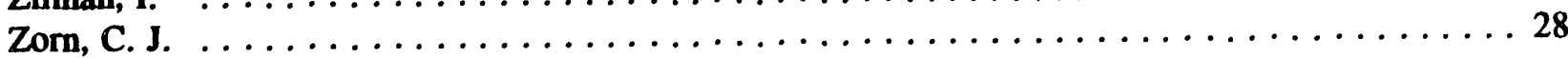



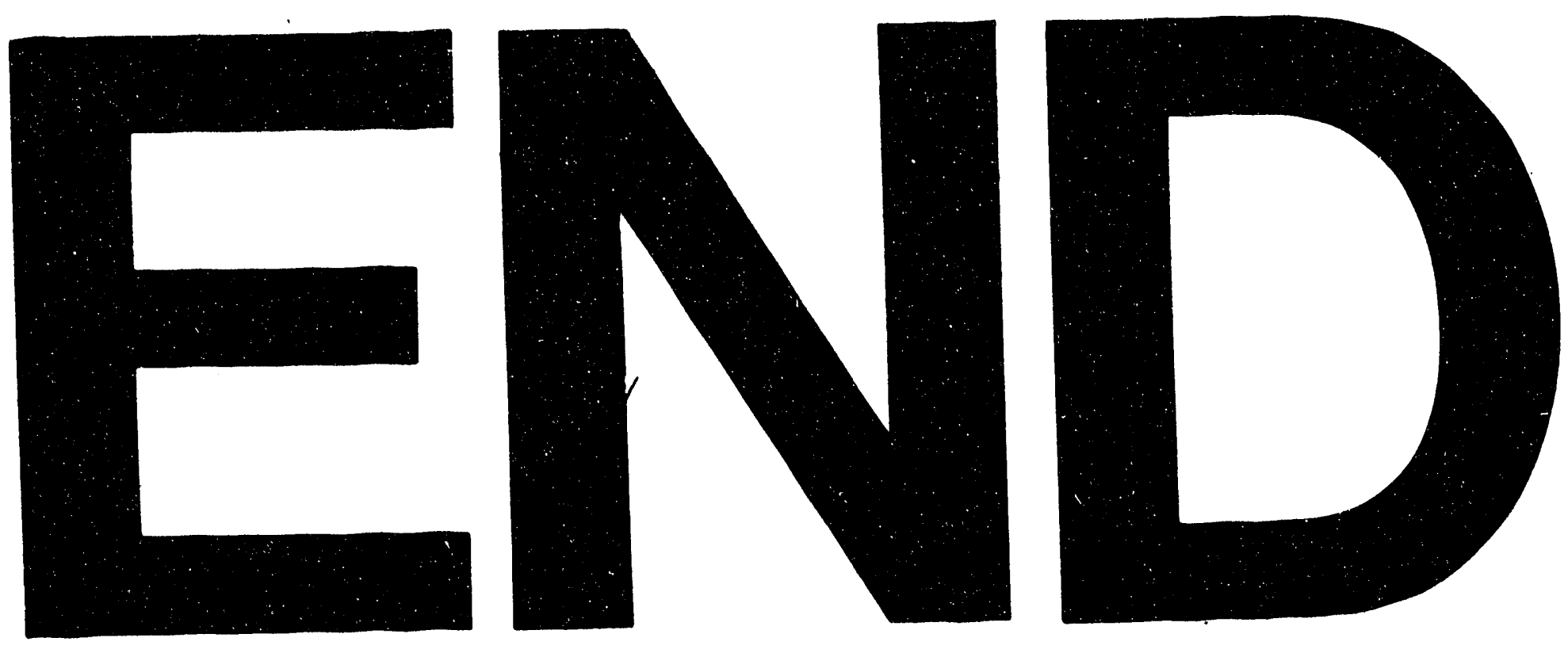

$t$ 